UNIVERSIDADE DE SÃO PAULO

ESCOLA DE ENGENHARIA DE SÃO CARLOS

DEPARTAMENTO DE ENGENHARIA ELÉTRICA

FABÍOLO MORAES AMARAL

Caracterização, Estimativas e Bifurcações da Região de

Estabilidade de Sistemas Dinâmicos

Não Lineares 



\section{Caracterização, Estimativas e Bifurcações da Região de Estabilidade de Sistemas Dinâmicos Não Lineares}

Tese de Doutorado apresentada à Escola de Engenharia de São Carlos da Universidade de São Paulo, como parte dos requisitos para obtenção do título de Doutor em Ciências, Programa Engenharia Elétrica.

Área de concentração: Sistemas Elétricos de Potência.

ORIENTADOR: Prof. Dr. Luís F. C. Alberto 
AUTORIZO A REPRODUÇÃO E DIVULGAÇÃO TOTAL OU PARCIAL DESTE TRABALHO, POR QUALQUER MEIO CONVENCIONAL OU ELETRÔNICO, PARA FINS DE ESTUDO E PESQUISA, DESDE QUE CITADA A FONTE.

Ficha catalográfica preparada pela Seção de Tratamento

da Informação do Serviço de Biblioteca - EESC/USP

Amaral, Fabíolo Moraes
Caracterização, estimativas e bifurcações da região de
estabilidade de sistemas dinâmicos não lineares/ Fabíolo
Moraes Amaral i orientador Luís Fernando Costa Alberto. -
- São Carlos, 2010.
Tese (Doutorado-Programa de Pós-Graduação em
Engenharia Elétrica e Área de Concentração em Sistemas
Elétricos Potência) -- Escola de Engenharia de São Carlos
da Universidade de São Paulo, 2010.
1. Sistemas não lineares. 2. Região de estabilidade.
3. Bacia de atração. 4. Fronteira da região de
estabilidade. 5. Estimativas da região de estabilidade.
6. Bifurcação sela-nó. I. Título.




\section{FOLHA DE JULGAMENTO}

Candidato(a): Bacharel FABÍOLO MORAES AMARAL.

Tese defendida e julgada em 24/09/2010 perante a Comissão Julgadora:

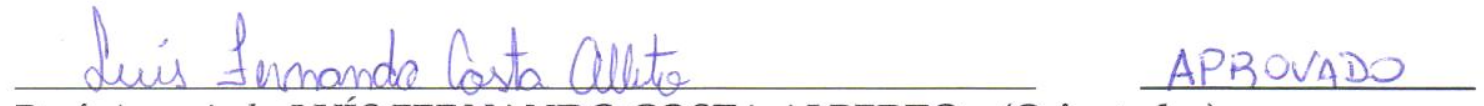

Prof. Associado LUÍS FERNANDO COSTA ALBERTO - (Orientador)

(Escola de Engenharia de São Carlos/USP)

Pavia do Camo Cauhinato

APROVADO

Profa. Associada MARIA DO CARMO CARBINATTO

(Instituto de Ciências Matemáticas e de Computação/USP)

Prof. Dr. ALEXANDRE SANFELICE BAZANELLA

APROVADO

(Universidade Federal do Rio Grando do Sul/UFRGS)
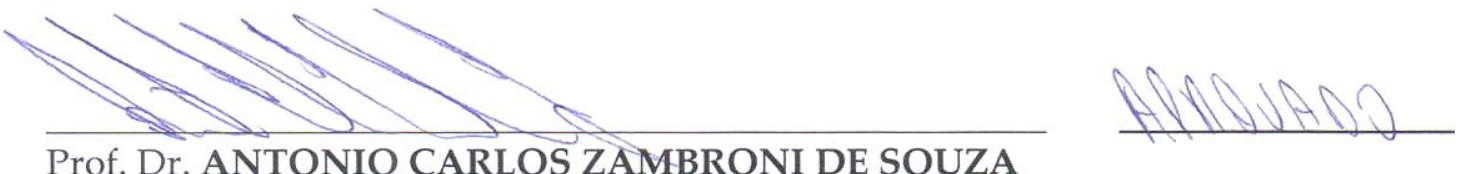

(Universidade Federal de Itajubá/UNIFEI)

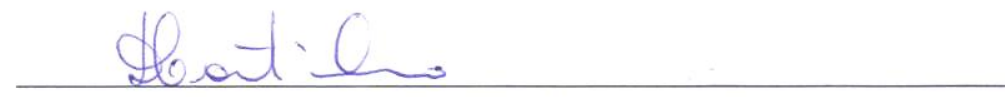

APHOVADO

Prof. Dr. DANIEL FERREIRA COUTINHO

(Pontifícia Universidade Católica do Rio Grande do Sul/PUC-RS)

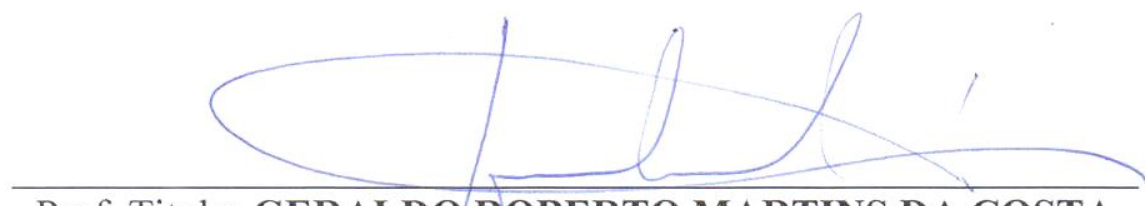

Prof. Titular GERALDO ROBERTO MARTINS DA COSTA

Coordenador do Programa de Pós-Graduação em Engenharia Elétrica e

Presidente da Comissão de Pós-Graduação 

Porque o Senhor ama o seu povo, e dá aos humildes a honra da vitória.

Salmo 149, versículo 4. 



\section{DEDICATÓRIA}

Dedico este trabalho às minhas avós, aos meus avôs, a minha mãe, ao meu pai e a minha esposa. 



\section{AGRADECIMENTOS}

A Deus.

A minha filha Vitória Teixeira Moraes Amaral pela sua existência.

Aos meus pais Relma Moraes Firmo Amaral e Orlando José Amaral Melo pelos ensinamentos e apoio incondicional ao longo da minha vida.

A minha esposa Renata Teixeira Moraes Amaral pelo seu amor e dedicação.

Aos meu irmãos Fabíola Moraes Amaral e Thiago Moraes Amaral pela amizade.

Ao orientador Luís Fernando Costa Alberto pela oportunidade de crescimento profissional e pela confiança a mim depositada.

Aos meus tios e primos pelo carinho.

Aos colegas de laboratório pelas oportunidades de trabalho em conjunto e descontração.

Aos professores do programa de pós-graduação em engenharia elétrica da Escola de Engenharia de São Carlos.

Ao Instituto Federal de Educação, Ciência e Tecnologia da Bahia, pelo apoio financeiro para a realização desta pesquisa. 



\section{RESUMO}

Estimar a região de estabilidade de um ponto de equilíbrio assintoticamente estável é importante em aplicações tais como sistemas de potência, economia e ecologia. A compreensão da estrutura qualitativa da fronteira da região de estabilidade é fundamental para estimar com eficiência a região de estabilidade. Caracterizações topológicas e dinâmicas da fronteira da região de estabilidade foram desenvolvidas ao longo das últimas décadas. Estas caracterizações foram desenvolvidas sob hipóteses de hiperbolicidade dos pontos de equilíbrio na fronteira e transversalidade. Para sistemas que dependem de parâmetros, a condição de hiperbolicidade pode ser violada em pontos de bifurcações. Estaremos interessados em estimar a região de estabilidade, para sistemas sujeitos a variações de parâmetros, onde ocorre a violação da condição de hiperbolicidade dos pontos de equilíbrio na fronteira da região de estabilidade devido ao aparecimento de uma bifurcação sela-nó do tipo zero nesta fronteira. Apresentaremos neste trabalho uma caracterização completa da fronteira da região de estabilidade na presença de um ponto de equilíbrio não hiperbólico sela-nó do tipo zero. Motivados também em oferecer um algoritmo conceitual para obter estimativas da região de estabilidade perturbada via conjunto de nível de uma dada função energia na vizinhança de um parâmetro de bifurcação sela-nó do tipo zero, buscaremos exibir resultados que permitam compreender o comportamento da região de estabilidade e de sua fronteira sob a influência das variações do parâmetro, incluindo variações do parâmetro próximo a um parâmetro de bifurcação sela-nó do tipo zero.

Palavras-chave: Sistemas não lineares, região de estabilidade, bacia de atração, fronteira da região de estabilidade, estimativas da região de estabilidade, bifurcação selanó. 



\begin{abstract}
Estimating the stability region of an asymptotically stable equilibrium point is fundamental in applications such as power systems, economy and ecology. The knowledge of the qualitative structure of the stability boundary is essential to estimate with efficiency the stability region. Topological and dynamical characterizations of the stability boundary have been developed over the past decades. These characterizations were developed under assumptions of hyperbolicity of equilibrium points on the stability boundary and transversality. For systems that depend on parameters, the condition of hyperbolicity can be violated at points of bifurcations. We will be primarily interested in estimating the stability region, for systems subjected to parameter variations, when the condition of hyperboli-city of equilibrium points on the stability boundary is violated due to the appearance of a type-zero saddle-node bifurcation on the stability boundary. We will develop in this work, a complete characterization of the stability boundary in the presence of a type-zero saddle-node non-hyperbolic equilibrium point. Also, motivated to providing a conceptual algorithm to obtain estimates of the perturbed stability region via level sets of a given energy function in the neighborhood of a type-zero saddle-node bifurcation parameter, we offer results that explain the behavior of the stability region and its boundary under the influence of parameter variations, including variations of the parameter close to a type-zero saddle-node bifurcation parameter.
\end{abstract}

Keywords: Nonlinear systems, Stability region, Basin of attraction, Stability boundary, Estimates of the stability region, Saddle-node bifurcation. 



\section{SUMÁRIO}

LISTA DE ILUSTRAÇÕES . . . . . . . . . . . . . . . . . . . . . . . . III

LISTA DE SímBOLOS . . . . . . . . . . . . . . . . . . . . . . . . . X XI

1 INTRODUÇÃo . . . . . . . . . . . . . . . . . . . . . . . 1

1.1 Estimativas da região de estabilidade . . . . . . . . . . . . . . . . . 1

1.2 Caracterização da fronteira da região de estabilidade . . . . . . . . . . . . 2

1.3 Objetivos ........................... 4

1.4 Contribuições ........................ 5

1.5 Organização do texto . . . . . . . . . . . . . . . . 5

2 PRELIMINARES . . . . . . . . . . . . . . . . . . 7

2.1 Espaço Euclidiano $\ldots \ldots \ldots \ldots \ldots$

$2.1 .1 \quad$ Norma . . . . . . . . . . . . . . . . 7

2.1 .2 Conjuntos Abertos . . . . . . . . . . . . . . . 8

2.1 .3 Conjuntos Fechados . . . . . . . . . . . . . . . . . 9

2.1 .4 Conjuntos Conexos . . . . . . . . . . . . . . . . 11

2.1.5 Conjuntos Contráteis . . . . . . . . . . . . . . 13

2.1 .6 Espaços Normais . . . . . . . . . . . . . . . 13

2.1.7 Distância entre dois conjuntos . . . . . . . . . . . . . . . . 14

2.2 Equações Diferenciais Ordinárias . . . . . . . . . . . . . . . . . . 15

2.2 .1 Teoria Geral . . . . . . . . . . . . . . . . . 15

2.2.2 Conjuntos Invariantes . . . . . . . . . . . . . . . . 16

2.2.3 Comportamento Assintótico _. . . . . . . . . . . . . . 17

2.2.4 Equilíbrios e Estabilidade Local . . . . . . . . . . . . . . . 20

2.2.5 Variedades Invariantes . . . . . . . . . . . . . . . . . . . 24

2.3 Transversalidade . . . . . . . . . . . . . . . . . 27

2.4 Propriedade genérica . . . . . . . . . . . . . . . . . 28 
3 REGIÃo de ESTABILIDADE $\ldots \ldots \ldots \ldots \ldots \ldots \ldots$

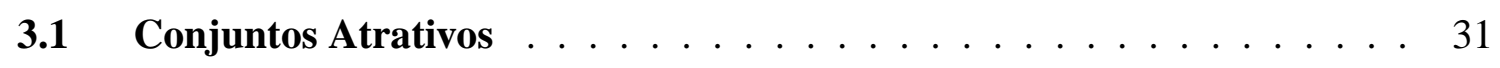

3.2 Caracterização topológica da região de estabilidade $\ldots \ldots \ldots \ldots 35$

4 CARACTERIZAÇÃo DA FRONTEIRA DA REGIÃO DE ESTABILIDADE 45

4.1 Pontos de equilíbrio na fronteira da região de estabilidade . . . . . . . 45

4.2 Estrutura dos pontos de equilíbrio na fronteira da região de estabilidade 49

5 CARACTERIZAÇÃO DA FRONTEIRA DA REGIÃO DE ESTABILIDADE NA PRESENÇA DE UM PONTO DE EQUILÍBRIO SELA-NÓ DO TIPO

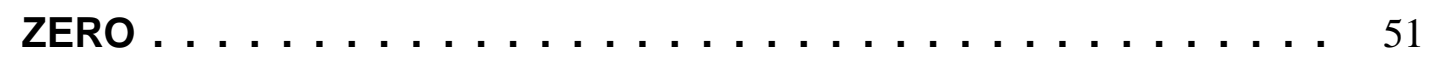

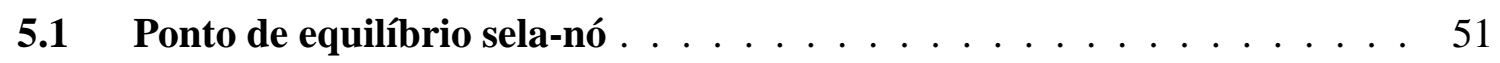

$5.2 \lambda$-Lema e equilíbrio sela-nó do tipo zero $\ldots \ldots \ldots \ldots \ldots$

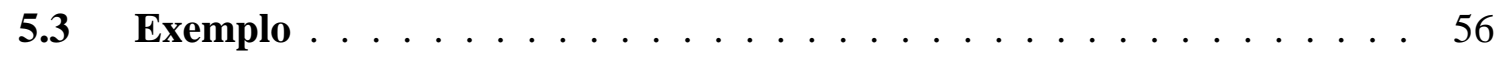

5.4 Lemas auxiliares $\ldots \ldots \ldots \ldots \ldots \ldots$

5.5 Ponto de equilíbrio sela-nó do tipo zero na fronteira da região de esta-

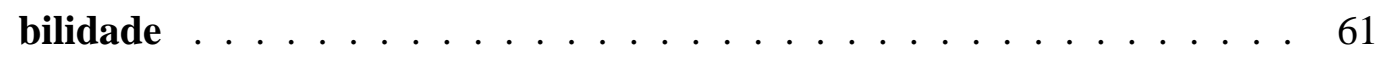

5.6 Região de estabilidade fraca $\ldots \ldots \ldots \ldots$. . . . . . . . . . 67

6 PERSISTÊNCIA DA REGIÃo DE ESTABILIDADE $\ldots \ldots \ldots$

6.1 Persistência dos pontos de equilíbrio hiperbólicos na fronteira da região

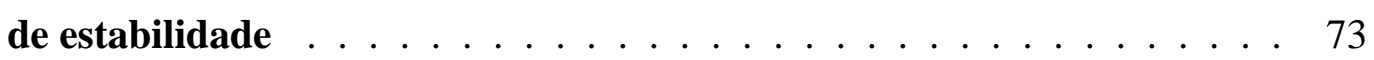

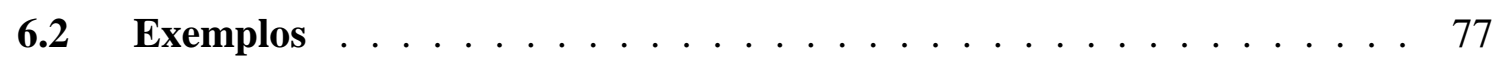

7 BIFURCAÇÃo A UM PARÂMETRO . . . . . . . . . . . . . . 81

7.1 Estabilidade Estrutural $\ldots \ldots \ldots \ldots \ldots$

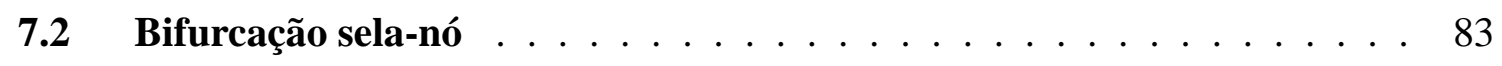

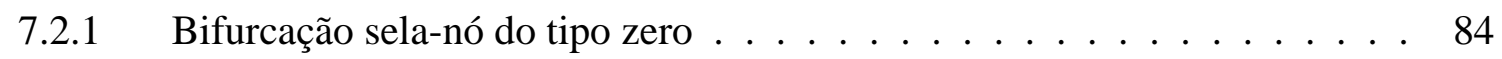

8 BIFURCAÇÃO SELA-NÓ DO TIPO ZERO NA FRONTEIRA DA REGIÃO DE ESTABILIDADE . . . . . . . . . . . . . . . . . . . 87

8.1 Comportamento da fronteira da região de estabilidade na presença de um ponto de equilíbrio sela-nó do tipo zero . . . . . . . . . . . . . . 87

8.2 Exemplos e Aplicações . . . . . . . . . . . . . . . . . . . . 95

9 ESTIMATIVAS DA REGIÃO DE ESTABILIDADE COM BIFURCAÇÕES SELA-NÓ DO TIPO ZERO NA FRONTEIRA . . . . . . . . . . . . 103

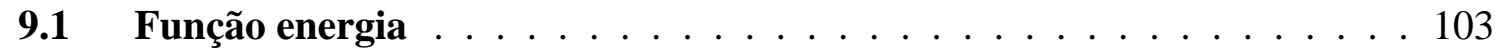

9.2 Estimativas da região de estabilidade . . . . . . . . . . . . . . . . . . 104 
9.3 Função energia e a caracterização da fronteira da região de estabilidade na presença de um ponto de equilíbrio sela-nó do tipo zero . . . . . . . . 106

9.4 Estimativas da região de estabilidade na presença de um ponto de equilíbrio sela-nó do tipo zero na fronteira . . . . . . . . . . . . . . . . 109

9.5 Comportamento das estimativas da região de estabilidade próximo a um parâmetro de bifurcação sela-nó do tipo zero . . . . . . . . . . . . . 113

10 Conclusões . . . . . . . . . . . . . . . . . . . . . . . 125

10.1 Trabalhos Futuros . . . . . . . . . . . . . . . . 126

REFERÊNCIAS . . . . . . . . . . . . . . . . . . . . . 127 



\section{LISTA DE ILUSTRAÇÕES}

Figura 1.1: Classificação dos métodos para estimar a região de estabilidade. . . . 2

Figura 2.1: $\quad p \in \Omega$ é um ponto $\omega$-limite e $\Omega$ é o conjunto $\omega$-limite da solução $\varphi(t, x) .18$

Figura 2.2: Conjunto $\omega$-limite desconexo e ilimitado. . . . . . . . . . . . . 20

Figura 2.3: Solução estável. . . . . . . . . . . . . . . . . . . . . . 20

Figura 2.4: Solução assintoticamente estável. . . . . . . . . . . . . . . . . . . . 21

Figura 2.5: Ponto de equilíbrio estável. . . . . . . . . . . . . . . . . . . 21

Figura 2.6: Ponto de equilíbrio atrativo. . . . . . . . . . . . . . . . 22

Figura 2.7: Ponto de equilíbrio assintoticamente estável. . . . . . . . . . . 23

Figura 2.8: Variedade estável $W^{s}$ e variedade instável $W^{u} \ldots \ldots \ldots$. . . . . . . 25

Figura 2.9: Retrato de fase do sistema (2.5) . . . . . . . . . . . . . . . . 26

Figura 2.10: Curvas $M$ e $N$ em $\mathbb{R}^{2} \ldots \ldots \ldots \ldots \ldots \ldots$

Figura 2.11: Ilustração do Exemplo 2.3.1 . . . . . . . . . . . . . . . . . . 28

Figura 2.12: Variedades não transversais. . . . . . . . . . . . . . . . 28

Figura 3.1: Vizinhança atrativa $U=U_{1} \cup U_{2}$ do conjunto atrativo $H=H_{1} \cup H_{2}$ do sistema (3.1) onde $H_{1}$ é um ciclo limite estável e $H_{2}=\{(0 ; 0)\}$ um ponto de equilíbrio instável. . . . . . . . . . . . . 33

Figura 3.2: Vizinhança atrativa $U$ do conjunto atrativo $H=[-1,1] \times\{0\}$ do sistema (3.2). . . . . . . . . . . . . . . . 34

Figura 3.3: Ponto de equilíbrio atrativo do sistema (3.3). . . . . . . . . . 35

Figura 3.4: Vizinhança atrativa $U=U_{1} \cup U_{2}$ do conjunto atrativo $H=\{(-1 ; 0),(1 ; 0)\}$ do sistema (3.2). . . . . . . . . . . . . . . . . . 38

Figura 3.5: Conexidade por caminho da região de estabilidade. . . . . . . . . . . 38

Figura 4.1: Retrato de fase do sistema (4.1) . . . . . . . . . . . . . . . 47

Figura 4.2: Retrato de fase do sistema (4.2) . . . . . . . . . . . . . . . 48

Figura 4.3: Retrato de fase do sistema (4.3) . . . . . . . . . . . . . . . . . . 49 
Figura 5.1: As variedades locais $W_{l o c}^{c^{+}}(p)$ e $W_{l o c}^{s}(p)$ são únicas, ao passo que existem infinitas escolhas para $W_{l o c}^{c^{-}}(p)$. Três possíveis escolhas para $W_{l o c}^{c^{-}}(p)$ são indicadas nesta figura. . . . . . . . . . . . 53

Figura 5.2: Vizinhança $U$ de um ponto de equilíbrio sela-nó do tipo zero $p$ do sistema (2.1). A parte cinza da figura corresponde ao conjunto $U^{-}$. .54

Figura 5.3: Ilustração do Lema 5.2.1. . . . . . . . . . . . . . . . . . . . . 55

Figura 5.4: Ilustração do Lema 5.2.2 e Corolário 5.2.2. A parte cinza da figura corres-ponde ao conjunto $\cup_{t \leqslant 0} \varphi\left(t, D^{s}\right) \ldots \ldots \ldots \ldots$

Figura 5.5: Ilustração do Lema 5.2.3 . . . . . . . . . . . . . . . . . . . . . . 57

Figura 5.6: O retrato de fase do sistema (5.1). A fronteira da região de estabilidade é constituída pela união da variedade estável do ponto de equilíbrio hiperbólico do tipo um $(1 ; 0)$, a curva em azul, com a variedade estável do ponto de equilíbrio sela-nó do tipo zero $(0 ;-1)$, a curva em vermelho. . . . . . . . . . . . . . . . . 58

Figura 5.7: Um ponto de equilíbrio sela-nó do tipo zero $p$ na fronteira da região de estabilidade de um ponto de equilíbrio assintoticamente estável $x^{s}$. A componente instável $W^{c^{+}}(p)$ da variedade central $W^{c}(p)$ intercepta a região de estabilidade $A\left(x^{s}\right)$, ao passo que $W^{s}(p)$ está contida na fronteira da região de estabilidade. . . . . . . . . . . . . . . 63

Figura 5.8: Exemplo de um sistema dinâmico onde a variedade $W^{c^{+}}(p)$ e a variedade estável $W^{s}(p)$ de um ponto de equilíbrio sela-nó do tipo zero $p$ não satisfazem a condição de transversalidade na fronteira da região de estabilidade de um ponto de equilíbrio as-sintoticamente estável $x^{s}$. 66

Figura 5.9: O retrato de fase do sistema (5.2). A fronteira da região de estabilidade do ponto de equilíbrio assintoticamente estável $(1,1547 ; 0)$ é formada pela variedade estável do ponto de equilíbrio sela-nó do tipo zero $(-0,5774 ; 0)$, a curva em azul. . . . . . . . . . . . 68

Figura 5.10: O retrato de fase do sistema (5.3). A fronteira da região de estabilidade fraca $\partial S(0 ; 0,25)$ é forma pela união da variedade estável do ponto de equilíbrio sela-nó do tipo zero $(0 ; 0,25)$, a curva em vermelho, com a variedade estável do ponto de equilíbrio $(1,11 ; 0,25)$, a curva em azul. . . . . . . . . . . . . . . . . 72

Figura 6.1: Retrato de fase do sistema (6.2) para $\lambda_{*}=0$. O ponto de equilíbrio instável $x_{\lambda^{*}}^{1}=-1$ pertence à fronteira da região de estabilidade do ponto de equilíbrio assintoticamente estável $x_{\lambda^{*}}^{s}=0 . \ldots \ldots 76$ 
Figura 6.2: Retrato de fase do sistema (6.2) para $\lambda=0,01$. O ponto de equilíbrio instável perturbado $x_{\lambda}^{1}=-0,980$ pertence à fronteira da região de estabilidade do ponto de equilíbrio assintoticamente estável $x_{\lambda}^{s}=0$. E o novo ponto de equilíbrio instável $x_{\lambda}^{2}=6,044$ também pertence à fronteira da região de estabilidade $\partial A_{\lambda}(0) . \ldots \ldots$. . . . . 76

Figura 6.3: Retrato de fase do sistema (6.3) para $\lambda=0,9$. A fronteira da região de estabilidade perturbada $\partial A_{\lambda}(-0,9487)$ do ponto de equilíbrio assintoticamente estável $x_{\lambda}^{s}=-0,9487$ é constituída pelo ponto de equilíbrio hiperbólico perturbado $x_{\lambda}^{1}=0,9487 \ldots \ldots \ldots \ldots$

Figura 6.4: Retrato de fase do sistema (6.3) para $\lambda_{*}=1$. A fronteira da região de estabi-lidade $\partial A_{\lambda_{*}}(-1)$ do ponto de equilíbrio assintoticamente estável $x_{\lambda^{*}}^{s}=-1$ é constituída pelo ponto de equilíbrio hiperbólico

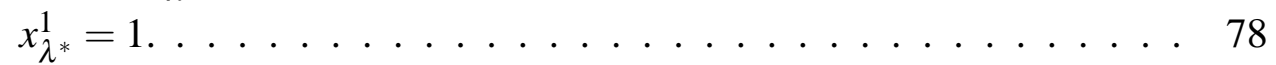

Figura 6.5: Retrato de fase do sistema (6.3) para $\lambda=1,1$. A fronteira da região de estabilidade perturbada $\partial A_{\lambda}(-1,0488)$ do ponto de equilíbrio assintoticamente estável $x_{\lambda}^{s}=-1,0488$ é constituída pelo ponto de equilíbrio hiperbólico perturbado $x_{\lambda}^{1}=1,0488 \ldots \ldots \ldots$. . . . . 78

Figura 6.6: Retrato de fase do sistema (6.4) para $\lambda=-0,1$. A fronteira da região de estabilidade perturbada $\partial A_{\lambda}(-2,0488 ;-1,0162)$ é a união das variedades estáveis dos pontos de equilíbrio $(-2,0488 ; 2,0162)$, $(0,0488 ; 0,0514)$ e $(0,0488 ; 0,9485)$, as curvas em azul.

Figura 6.7: Retrato de fase do sistema (6.4) para $\lambda_{*}=0$. A fronteira da região de estabi-lidade $\partial A_{\lambda_{*}}(-2 ;-1)$ é a união das variedades estáveis dos pontos de equilíbrio $(-2 ; 2),(0 ; 1)$ e $(0 ; 0)$, as curvas em azul. . . . . 79

Figura 6.8: Retrato de fase do sistema (6.4) para $\lambda=0,1$. A fronteira da região de estabilidade perturbada $\partial A_{\lambda}(-1,9487 ;-0,9828)$ é a união das variedades estáveis dos pontos de equilíbrio $(-1,9487 ; 1,98282),(-0,0513 ;-0,0489)$ e $(-0,0513 ; 1,0489)$, as curvas em azul. . . . . . . . . . 80

Figura 7.1: Retrato de fase dos campos vetoriais $f$ e $g$. . . . . . . . . . 82

Figura 7.2: Retratos de fase do sistema (7.2) . . . . . . . . . . . . . 83

Figura 7.3: Retrato de fase do sistema (7.1) na vizinhança $N$ do Teorema 7.2.2 para $\lambda \in\left(\lambda_{0}-\delta, \lambda_{0}+\delta\right)$. Para $\lambda<\lambda_{0}$, o sistema (7.1) tem dois pontos de equilíbrio hiperbólicos, um ponto de equilíbrio do tipo 0 e um do tipo 1. Em $\lambda=\lambda_{0}$, o sistema (7.1) tem um único ponto de equilíbrio $x_{\lambda_{0}}$ que é um ponto de equilíbrio sela-nó do tipo zero. Para $\lambda>\lambda_{0}$, o sistema (7.1) não tem ponto de equilíbrio em $N$. . . . . . 85

Figura 7.4: Vizinhança $U$ de um ponto de equilíbrio sela-nó do tipo zero $x_{\lambda_{0}}$ para $\lambda<\lambda_{0}$. A parte cinza da figura corresponde ao conjunto $U_{\lambda}^{-} \ldots \ldots 86$ 
Figura 8.1: Comportamento da vizinhança $U$ de um ponto de equilíbrio sela-nó do tipo zero $x_{\lambda_{0}}$ que está na fronteira da região de estabilidade de um ponto de equilíbrio assintoticamente estável $x_{\lambda_{0}}^{s} \ldots \ldots \ldots 8$

Figura 8.2: Região de estabilidade do sistema (7.1) para $\lambda<\lambda_{0}$. A área em roza mais escuro é a região de estabilidade do ponto de equilíbrio assintoticamente estável $y_{\lambda}^{s}$ enquanto a área em roza mais claro é a região de estabilidade de um ponto de equilíbrio assintoticamente estável $x_{\lambda}^{s} . \quad 94$

Figura 8.3: Região de estabilidade do sistema (7.1) para $\lambda=\lambda_{0}$. O ponto de equilíbrio assintoticamente estável $y_{\lambda}^{s}$ da Figura 8.2 coalesceu com o ponto de equilíbrio do tipo um $y_{\lambda}^{u}$ em um ponto de equilíbrio sela-nó do tipo zero $x_{\lambda_{0}}$. O ponto de equilíbrio sela-nó do tipo zero $x_{\lambda_{0}}$ está na fronteira da região de estabilidade de $x_{\lambda_{0}}^{s}$ e a área em roza mais escuro é a região de estabilidade fraca de $x_{\lambda_{0}} \ldots \ldots \ldots$. . . . . . . . 94

Figura 8.4: Região de estabilidade do sistema (7.1) para $\lambda>\lambda_{0}$. O ponto de equilíbrio hiperbólico do tipo um $x_{\lambda}^{*}$ que pertencia a fronteira da região de estabilidade de $y_{\lambda}^{s}$ para $\lambda<\lambda_{0}$ agora pertence a fronteira da região de estabilidade de $x_{\lambda}^{s}$. O ponto de equilíbrio $x_{\lambda}^{s}$ "herda" toda a região de estabilidade do ponto de equilíbrio $y_{\lambda}^{s}$, que desapareceu. . . . . . . 94

Figura 8.5: $\quad$ O retrato de fase do sistema (8.1) para $\lambda=-1,02$. A região de estabilidade do ponto de equilíbrio assintoticamente estável $(-0,99 ;-0,02)$ é representada pela área em cinza. A fronteira da região de estabilidade é constituída pela união da variedade estável do ponto de equilíbrio hiperbólico instável $(-0,2 ;-0,97)$, originado da bifurcação sela-nó do tipo zero, a curva em vermelho, com a variedade estável do ponto de equilíbrio hiperbólico $(0,99 ;-0,02)$, a curva em azul. . . 98

Figura 8.6: $O$ retrato de fase do sistema (8.1) para $\lambda_{0}=-1$. A região de estabilidade do ponto de equilíbrio assintoticamente estável $(-1 ; 0)$ é representada pela área em cinza. A fronteira da região de estabilidade é constituída pela união da variedade estável do ponto de equilíbrio sela-nó do tipo zero $(0 ;-1)$, a curva em vermelho, com a variedade estável do ponto de equilíbrio hiperbólico $(1 ; 0)$, a curva em azul. . . 98

Figura 8.7: $\mathrm{O}$ retrato de fase do sistema (8.1) para $\lambda=-0,98$. A região de estabilidade do ponto de equilíbrio assintoticamente estável $(-0,99 ; 0,01)$ é representada pela área em cinza. A fronteira da região de estabilidade é constituída pela variedade estável do ponto de equilíbrio hiperbólico $(0,99 ; 0,01)$, a curva em azul . . . . . . . . . . . 99 
Figura 8.8: O retrato de fase do sistema (8.2) para $\lambda=0,2$. A região de estabilidade do ponto de equilíbrio assintoticamente estável $(-1,09 ; 0,2)$ é representada pela área em cinza escuro. A região de estabilidade do ponto de equilíbrio hiperbólico assintoticamente estável $(0,2 ; 0,2)$, originado da bifurcação sela-nó do tipo zero, é representada pela área em cinza claro. A fronteira da região de estabilidade $\partial A_{\lambda}(-1,09 ; 0,2)$ é constituída pela variedade estável do ponto de equilíbrio hiperbólico instável $(-0,2 ; 0,2)$, originado da bifurcação sela-nó do tipo zero, a curva em vermelho. A fronteira da região de estabilidade $\partial A_{\lambda}(0,2 ; 0,2)$ é constituída pela união da variedade estável do ponto de equilíbrio $(-0,2 ; 0,2)$, com a variedade estável do ponto de equilíbrio hiperbólico $(1,09 ; 0,2)$, a curva em azul. . . . . . . . . . . . . 99

Figura 8.9: $O$ retrato de fase do sistema (8.2) para $\lambda_{0}=0,25$. A região de estabilidade do ponto de equilíbrio assintoticamente estável $(-1,11 ; 0,25)$ é representada pela área em cinza escuro. A região de estabilidade fraca de $(0 ; 0,25)$ é representada pela área em cinza claro. A fronteira da região de estabilidade $\partial A_{\lambda_{0}}(-1,11 ; 0,25)$ é constituída pela variedade estável do ponto de equilíbrio sela-nó do tipo zero $(0 ;, 0,25)$, a curva em vermelho. A fronteira da região de estabilidade fraca $\partial S_{\lambda_{0}}(0 ; 0,25)$ é constituída pela união da variedade estável do ponto de equilíbrio sela-nó do tipo zero, com a variedade estável do ponto de equilíbrio hiperbólico $(1,11 ; 0,25)$, a curva em azul. . . . . . . . 100

Figura 8.10: $O$ retrato de fase do sistema (8.2) para $\lambda=0,3$. A região de estabilidade do ponto de equilíbrio hiperbólico assintoticamente estável $(-1,13 ; 0,3)$ é representada pela área em cinza escuro. A fronteira da região de estabilidade $\partial A_{\lambda}(-1,13 ; 0,2)$ é constituída pela variedade estável do ponto de equilíbrio hiperbólico instável $(1,13 ; 0,3)$, a curva em azul. . . . . . . . . . . . . . . . . 100

Figura 8.11: Diagrama de bifurcação do sistema (8.3). Este gráfico descreve a componente $x_{1}$ dos pontos de equilíbrio de (8.3) como uma função da entrada $I_{1}$. A linha tracejada corresponde a coordenada dos pontos de equilíbrio do tipo um enquanto a linha contínua indica os pontos de equilíbrio assintoticamente estáveis. Uma bifurcação sela-nó do tipo zero ocorre em $I_{1}=0,418$. Se o parâmetro $I_{1}$ cresce lentamente, então, para o valor de entrada 0,418 , a saída da rede salta rapidamente do ponto de equilíbrio sela-nó do tipo zero $(-1,44 ;-0,51)$ para o ponto de equilíbrio assintoticamente estável $(3,69 ; 0,95) \ldots$. . . . . 101 
Figura 8.12: $O$ retrato de fase do sistema (8.3) para $I_{1}=0,4$. A região de estabilidade do ponto de equilíbrio assintoticamente estável $(3,66 ; 0,95)$ é representada pela área em cinza. A fronteira da região de estabilidade é constituída pela variedade estável do ponto de equilíbrio hiperbólico instável $(-1,17 ;-0,42)$, originado da bifurcação sela-nó do tipo zero, a curva em azul. . . . . . . . . . . . . . . . 101

Figura 8.13: O retrato de fase do sistema (8.3) para $I_{1}=0,418$. A região de estabilidade do ponto de equilíbrio assintoticamente estável $(3,69 ; 0,95)$ é representada pela área em cinza. A fronteira da região de estabilidade é constituída pela variedade estável do ponto de equilíbrio sela-nó do tipo zero $(-1,44 ;-0,51)$, a curva em azul. . . . . . . . . . 102

Figura 8.14: $O$ retrato de fase do sistema (8.3) para $I_{1}=0,43$. O ponto de equilíbrio $(3,71 ; 0,95)$ é globalmente assintoticamente estável. . . . . . 102

Figura 9.1: O retrato de fase do sistema (9.1). A região de estabilidade do ponto de equilíbrio assintoticamente estável $(-0,2 ; 0)$ é representada pela área em cinza claro. A componente conexa $D(0,39)$ do conjunto de nível $\left\{(x, y) \in \mathbb{R}^{2}: V(x ; y)<0,39\right\}$ contendo o equilíbrio $(-0,2 ; 0)$ representada pela área em cinza escuro é uma estimativa da região de estabilidade.

Figura 9.2: $\quad$ O retrato de fase do sistema (9.1). A região de estabilidade do ponto de equilíbrio assintoticamente estável $(-0,2 ; 0)$ é representada pela área em cinza claro. A componente conexa $D(0,41)$ do conjunto de nível $\left\{(x, y) \in \mathbb{R}^{2}: V(x ; y)<0,41\right\}$ contendo o equilíbrio $(-0,2 ; 0)$ representada pela área em cinza escuro intercepta o complementar da região de estabilidade $A^{c}(-0,2 ; 0)$. . . . . . . . . . . . . . 107

Figura 9.3: O retrato de fase do sistema (9.2). A região de estabilidade do ponto de equilíbrio assintoticamente estável $(3,43 ;-0,49)$ é representada pela área em cinza claro. A componente conexa $D(-1,55)$ do conjunto de nível $\left\{(x, y) \in \mathbb{R}^{2}: V(x ; y)<-1,55\right\}$ contendo o equilíbrio $(3,43 ;-0,49)$ representada pela área em cinza escuro é uma estimativa da região de estabilidade. . . . . . . . . . . . . . . . 112

Figura 9.4: O retrato de fase do sistema (9.2). A região de estabilidade do ponto de equilíbrio assintoticamente estável $(3,43 ;-0,49)$ é representada pela área em cinza claro. A componente conexa $D(-1,4)$ do conjunto de nível $\left\{(x, y) \in \mathbb{R}^{2}: V(x ; y)<-1,4\right\}$ contendo o equilíbrio $(3,43 ;-0,49)$ representada pela área em cinza escuro intercepta o complementar da região de estabilidade $A^{c}(3,43 ;-0,49)$. . . . . . 112 
Figura 9.5: $\quad$ O retrato de fase do sistema (9.3). A região de estabilidade do ponto de equilíbrio assintoticamente estável $(0,31 ; 0)$ é representada pela área em cinza claro. A componente conexa $D(0)$ do conjunto de nível $\left\{(x, y) \in \mathbb{R}^{2}: V(x ; y)<0\right\}$ contendo o equilíbrio $(0,31 ; 0)$ representada pela área em cinza escuro é uma estimativa da região de estabilidade.

Figura 9.6: O retrato de fase do sistema (9.3). A região de estabilidade do ponto de equilíbrio assintoticamente estável $(0,31 ; 0)$ é representada pela área em cinza claro. A componente conexa $D(0,2)$ do conjunto de nível $\left\{(x, y) \in \mathbb{R}^{2}: V(x ; y)<0,2\right\}$ contendo o equilíbrio $(0,31 ; 0)$ representada pela área em cinza escuro intercepta o complementar da região de estabilidade $A^{c}(0,31 ; 0) \ldots \ldots$. . . . . . . . . . . . . 113

Figura 9.7: Retrato de fase do sistema (9.4) para $\lambda=-0,1$. A região de estabilidade do ponto de equilíbrio assintoticamente estável $(-0,94 ; 0)$ é representada pela área em cinza claro. A componente conexa $D(-0,09)$ do conjunto de nível $\left\{(x, y) \in \mathbb{R}^{2}: V_{\lambda}(x ; y)<-0,09\right\}$ contendo o equilíbrio $(-0,94 ; 0)$ representada pela área em cinza escuro é uma

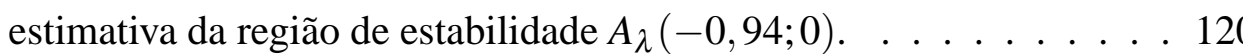

Figura 9.8: Retrato de fase do sistema (9.4) para $\lambda_{0}=0$. A região de estabilidade do ponto de equilíbrio assintoticamente estável $(-1 ; 0)$ é representada pela área em cinza claro. A componente conexa $D(0)$ do conjunto de nível $\left\{(x, y) \in \mathbb{R}^{2}: V_{\lambda_{0}}(x ; y)<0\right\}$ contendo o equilíbrio $(-1 ; 0)$ representada pela área em cinza escuro é uma estimativa da região de estabilidade $A_{\lambda_{0}}(-1 ; 0)$

Figura 9.9: Retrato de fase do sistema (9.4) para $\lambda=0,1$. A região de estabilidade do ponto de equilíbrio assintoticamente estável $(-1,04 ; 0)$ é representada pela área em cinza claro. A componente conexa $D(0)$ do conjunto de nível $\left\{(x, y) \in \mathbb{R}^{2}: V_{\lambda_{0}}(x ; y)<0\right\}$ contendo o equilíbrio $(-1,04 ; 0)$ representada pela área em cinza escuro é uma estimativa uniforme da região de estabilidade perturbada $A_{\lambda}(-1,04 ; 0) \ldots$. . . . 121

Figura 9.10: $\mathrm{O}$ retrato de fase do (9.5) para $\lambda=0,48$. A região de estabilidade do ponto de equilíbrio assintoticamente estável $(3,43 ;-0,48)$ é representada pela área em cinza claro. A componente conexa $D(-1,47)$ do conjunto de nível $\left\{(x, y) \in \mathbb{R}^{2}: V_{\lambda}(x ; y)<-1,47\right\}$ contendo o equilíbrio $(3,43 ;-0,48)$ representada pela área em cinza escuro é uma estimativa da região de estabilidade $A_{\lambda}(3,43 ;-0,48) \ldots \ldots$. . . 122 
Figura 9.11: $O$ retrato de fase do (9.5) para $\lambda_{0}=0,49$. A região de estabilidade do ponto de equilíbrio assintoticamente estável $(3,43 ;-0,49)$ é representada pela área em cinza claro. A componente conexa $D(-1,55)$ do conjunto de nível $\left\{(x, y) \in \mathbb{R}^{2}: V_{\lambda_{0}}(x ; y)<-1,55\right\}$ contendo o equilíbrio $(3,43 ;-0,49)$ representada pela área em cinza escuro é uma estimativa da região de estabilidade $A_{\lambda_{0}}(3,43 ;-0,49) . \quad$. . . . . 122

Figura 9.12: $O$ retrato de fase do (9.5) para $\lambda=0,51$. A região de estabilidade do ponto de equilíbrio assintoticamente estável $(3,43 ;-0,51)$ é representada pela área em cinza claro. A componente conexa $D(-1,64)$ do conjunto de nível $\left\{(x, y) \in \mathbb{R}^{2}: V_{\lambda}(x ; y)<-1,64\right\}$ contendo o equilíbrio $(3,43 ;-0,51)$ representada pela área em cinza escuro é uma estimativa da região de estabilidade $A_{\lambda}(3,43 ;-0,51) \ldots \ldots 123$

Figura 9.13: O retrato de fase do sistema (9.6) para $\lambda=-0,02$. A região de estabilidade do ponto de equilíbrio assintoticamente estável $(0,32 ; 0)$ é representada pela área em cinza claro. A componente conexa $D(-0,03)$ do conjunto de nível $\left\{(x, y) \in \mathbb{R}^{2}: V_{\lambda}(x ; y)<-0,03\right\}$ contendo o equilíbrio $(0,31 ; 0)$ representada pela área em cinza escuro é uma estimativa da região de estabilidade $A_{\lambda}(0,32 ; 0)$. . . . . . . . . 123

Figura 9.14: O retrato de fase do sistema (9.6) para $\lambda_{0}=0$. A região de estabilidade do ponto de equilíbrio assintoticamente estável $(0,31 ; 0)$ é representada pela área em cinza claro. A componente conexa $D(0)$ do conjunto de nível $\left\{(x, y) \in \mathbb{R}^{2}: V_{\lambda_{0}}(x ; y)<0\right\}$ contendo o equilíbrio $(0,31 ; 0)$ representada pela área em cinza escuro é uma estimativa da região de estabilidade $A_{\lambda_{0}}(0,31 ; 0) . \ldots \ldots$. . . . . . . . . 124

Figura 9.15: O retrato de fase do sistema (9.6) para $\lambda=0,02$. A região de estabilidade do ponto de equilíbrio assintoticamente estável $(0,3 ; 0)$ é representada pela área em cinza claro. A componente conexa $D(0)$ do conjunto de nível $\left\{(x, y) \in \mathbb{R}^{2}: V_{\lambda_{0}}(x ; y)<0\right\}$ contendo o equilíbrio $(0,3 ; 0)$ representada pela área em cinza escuro é uma estimativa uniforme da região de estabilidade perturbada $A_{\lambda}(0,3 ; 0) . \quad$. . . . . . 124 


\section{LISTA DE SÍMBOLOS}

$\lambda_{0} \quad$ Parâmetro de bifurcação sela-nó do tipo zero

$x^{s} \quad$ Ponto de equilíbrio assintoticamente estável

$A\left(x^{s}\right) \quad$ Região de estabilidade de $x^{s}$

$\overline{A\left(x^{s}\right)} \quad$ Fecho da região de estabilidade de $x^{s}$

$\partial A\left(x^{s}\right) \quad$ Fronteira da Região de estabilidade

$S(p) \quad$ Região de estabilidade fraca de um ponto de equilíbrio sela-nó do tipo zero $p$

$\partial S(p) \quad$ Fronteira da região de estabilidade fraca de um ponto de equilíbrio sela-nó do tipo zero $p$

E $\quad$ Conjunto dos pontos de equilíbrio

$\Omega(L) \quad$ Conjunto de nível $L$

$\partial B^{u} \quad$ Domínio fundamental da variedade instável

$N^{u} \quad$ Vizinhança fundamental da variedade instável 



\section{INTRODUÇÃO}

\subsection{Estimativas da região de estabilidade}

Pontos de equilíbrio assintoticamente estáveis de sistemas dinâmicos autônomos não lineares não são, em geral, globalmente estáveis. Na maioria dos casos, existe um subconjunto de condições iniciais, chamado de região de estabilidade, cujas trajetórias, iniciando dentro deste conjunto, tendem para o ponto de equilíbrio assintoticamente estável quando o tempo tende ao infinito. O problema de determinar a região de estabilidade de um ponto de equilíbrio assintoticamente estável para um sistema dinâmico autônomo não linear é relevante em diversas aplicações no campo da engenharia, incluindo problemas de estabilidade em sistemas elétricos de potência, (EL-ABIAD; NAGAPPAN, 1966), (YU; VONGSURIYA, 1967), (SARKAR; RAO, 1971), (N. KAKIMOTO; HAYASHI, 1978), (VARAIYA; WU; CHEN, 1985), (CHIANG; WU; VARAIYA, 1987), (PAI, 1981), (CHIANG; WU; VARAIYA, 1992), (COUTINHO; PAGANO; TROFINO, 2004), (COUTINHO et al., 2004), (COUTINHO; SOUZA; TROFINO, 2009), (DEMARCO; CANIZARES, 1992), (SILVA et al., 2009), (SILVA et al., 2005), (GUEDES; ALBERTO; BRETAS, 2005), análise dinâmica em reatores químicos (E. NOLDUS J. SPRIET; CAUWENBERGHE, 1974), técnicas de otimização global via sistemas dinâmicos (LEE; CHIANG, 2000) e em outras áreas tais como ecologia (MAY, 1973; GATTO; RINALDI, 1975) e economia (ARROW; HAHN, 1971). Os inúmeros métodos, propostos na literatura, para estimar a região de estabilidade de um ponto de equilíbrio assintoticamente estável podem ser grosseiramente divididos em duas classes (GENESIO; TARTAGLIA; VICINO, 1985): aqueles que usam função escalares tais como funções de Lyapunov ou funções energia, e aqueles que não usam funções escalares, ver Figura 1.1. A maioria dos métodos que utilizam funções escalares é baseada nos resultados de LaSalle que estendem a teoria de Lyapunov (LASALLE, 1960a,b; LASALLE; LEFSCHETZ, 1961). Funções escalares podem fornecer estimativas da região de estabilidade por meio de conjuntos de nível, onde estes são determinados por um número escalar. Sua utilização como estimativa tem a vantagem de ser simples, permitindo verificar se um ponto está dentro da região de estabilidade apenas verificando uma desigualdade entre números reais. Porém, os conjuntos 
de nível podem ser uma estimativa bastante conservadora da região de estabilidade, isto é, na maioria dos casos é somente um subconjunto da verdadeira região de estabilidade. Outro método que utiliza funções do tipo Lyapunov é o método de Zubov (ZUBOV, 1962, 1964, 1967). Teoricamente, este método fornece a verdadeira região de estabilidade via a solução de uma equação diferencial parcial. Na prática, este método pode ser inviável devido a convergência não uniforme do algoritmo, além disso ele requer um grande esforço computacional, como descrito no trabalho de Genesio, Tartaglia e Vicino (1985). Para sistemas planares, métodos que não se baseiam em funções escalares foram desenvolvidos, ver por exemplo (JOCIC, 1982) e (INFANTE; CLARK, 1964). Os métodos que não se baseiam em funções escalares, usualmente empregam o método da trajetória reversa. Neste método, a estimativa da região de estabilidade é sintetizada a partir de um certo número de trajetórias do sistema obtido pela integração numérica das equações do sistema para tempos negativos (GENESIO; TARTAGLIA; VICINO, 1985; GENESIO; VICINO, 1984). A desvantagem deste método é que para sistemas de ordem elevada exige-se um grande esforço computacional associado às rotinas de integração numérica do algoritmo, porém para sistemas de ordem reduzida, o método é viável oferecendo um estimativa quase exata da região de estabilidade como descrito no trabalho de Genesio, Tartaglia e Vicino (1985). No trabalho de M. Loccufier e E. Noldus (2000), um novo método de trajetória reversa foi proposto.

Neste trabalho, os métodos que serão utilizados para estimar a região usam funções escalares.

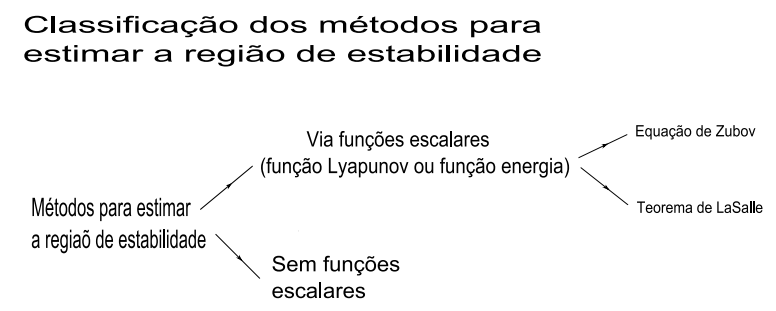

Figura 1.1: Classificação dos métodos para estimar a região de estabilidade.

\subsection{Caracterização da fronteira da região de estabilidade}

Muitos métodos foram propostos na literatura com o objetivo de estimar a região de estabilidade. Esses métodos começaram a ser largamente estudados na década de 60, motivados pelos estudos de estabilidade transitória em sistemas elétricos de potência, ver por exemplo (GLESS, 1966; EL-ABIAD; NAGAPPAN, 1966; WILLEMS, 1968). A maioria dos métodos usados na análise de estabilidade transitória em sistemas elétricos de potência, baseavam-se no Princípio de Invariância de LaSalle (LASALLE, 1960b) e não exploravam a estrutura qualitativa da fronteira da região de estabilidade. Além de serem bastantes conservadores, estes métodos eram heurísticos, e consequentemente sujeitos a 
erros.

Motivados em compreender a fronteira da região de estabilidade para obter estimativas ótimas da região de estabilidade na análise de estabilidade transitória em sistemas elétricos de potência, Tsolas, Arapostathis e Varaiya (1985) propõem na literatura uma primeira caracterização dinâmica da fronteira da região de estabilidade de um ponto de equilíbrio assintoticamente estável $x^{s}$, para um sistema dinâmico autônomo não linear

$$
\dot{x}=f(x)
$$

onde $f: \mathbb{R}^{n} \longrightarrow \mathbb{R}^{n}$ é um campo vetorial de classe $C^{1}$.

No trabalho de Tsolas, Arapostathis e Varaiya (1985), foi mostrado, sob algumas condições, que a fronteira da região de estabilidade é a união do fecho das variedades estáveis de todos os pontos de equilíbrio do tipo 1 que pertencem à fronteira da região de estabilidade.

Chiang, Wu e Varaiya (1987) apresentaram uma fundamentação teórica de métodos diretos para efetuar a estimativa da região de estabilidade. Esta fundamentação tem por base uma nova caracterização dinâmica da fronteira da região de estabilidade de um ponto de equilíbrio assintoticamente estável que generaliza a caracterização proposta por Tsolas, Arapostathis e Varaiya (1985). No trabalho de Chiang, Wu e Varaiya (1987), mostrou-se sob as condições

(A1) Todos pontos de equilíbrio na fronteira da região de estabilidade são hiperbólicos.

(A2) As variedades estáveis e instáveis dos pontos de equilíbrio na fronteira da região de estabilidade satisfazem a condição de transversalidade.

(A3) Toda trajetória na fronteira da região de estabilidade se aproxima de um ponto de equilíbrio quando $t \rightarrow+\infty$,

que a fronteira da região de estabilidade é a união das variedades estáveis de todos os pontos de equilíbrio na fronteira. Além disso, foram dadas condições necessárias e suficientes para um ponto de equilíbrio hiperbólico pertencer à fronteira da região de estabilidade.

Um pouco mais tarde, no trabalho de Chiang, Hirsch e Wu (1988) foi apresentada uma caracterização mais completa da fronteira da região de estabilidade de um ponto de equilíbrio assintoticamente estável que generaliza a caracterização proposta por Chiang, Wu e Varaiya (1987). Naquele trabalho, sob as condições

(B1) Todos pontos de equilíbrio(e/ou órbitas fechada) na fronteira da região de estabilidade são hiperbólicos.

(B2) As variedades estáveis e instáveis dos pontos de equilíbrio(e/ou órbitas fechada) na fronteira da região de estabilidade satisfazem a condição de transversalidade. 
(B3) Toda trajetória na fronteira da região de estabilidade se aproxima de um ponto de equilíbrio (e/ou órbitas fechada) quando $t \rightarrow+\infty$,

mostrou-se que a fronteira da região de estabilidade é a união das variedades estáveis de todos os pontos de equilíbrio (e/ou órbitas fechadas) na fronteira da região de estabilidade. Além disso, foram dadas condições necessárias e suficientes para um ponto de equilíbrio (e/ou órbitas fechadas) pertencer à fronteira da região de estabilidade.

Chiang, Wu e Varaiya (1988) apresentaram uma caracterização da fronteira da região de estabilidade para duas classes de sistemas dinâmicos: sistemas gradientes generalizados e sistemas descritos por uma equação diferencial vetorial de segunda ordem. A caracterização para estas duas classes específicas de sistemas foi a mesma dada por Chiang, Wu e Varaiya (1987), porém, para estes sistemas, apenas a hipótese (A2) foi utilizada para a caracterização da fronteira. Além disso, para estes sistemas particulares, mostrouse que os pontos de equilíbrio hiperbólicos na fronteira da região de estabilidade persistem na fronteira sob pequenas pertubações do campo vetorial.

Mais tarde, no trabalho de Chiang e Chia-Chu (1995), foram mostrados, sob as suposições $(A 1)-(A 3)$, para sistemas dinâmicos autônomos mais gerais, que os pontos de equilíbrio hiperbólicos na fronteira da região de estabilidade de um ponto de equilíbrio assintoticamente estável também persistem na fronteira sob pequenas pertubações do campo vetorial.

\subsection{Objetivos}

As suposições $(A 1)-(A 3)$ são fundamentais na caracterização da fronteira da região de estabilidade de um ponto de equilíbrio assintoticamente estável proposta por Chiang, Wu e Varaiya (1987). Mas, para sistemas que dependem de parâmetros, é muito comum a violação da suposição $(A 1)$ devido ao aparecimento de bifurcações na fronteira da região de estabilidade. Na análise de estabilidade de tensão em sistemas elétricos de potência, por exemplo, verificou-se a existência de bifurcações sela-nó na fronteira da região de estabilidade, violando assim a suposição (A1) (GUEDES; ALBERTO; BRETAS, 2005). Em vista disto, com o intuito de entender o comportamento da região de estabilidade de um ponto de equilíbrio assintoticamente estável e sua fronteira quando a suposição $(A 1)$ é violada pela ocorrência de bifurcações na fronteira da região de estabilidade, estuda-se neste trabalho o sistema de equações diferencias dependente de um parâmetro escalar

$$
\dot{x}=f(x, \lambda)
$$

com $x \in \mathbb{R}^{n}, \lambda \in \mathbb{R}$ e $f: \mathbb{R}^{n} \times \mathbb{R} \longrightarrow \mathbb{R}^{n}$ sendo um campo vetorial de classe $C^{1}$.

Como o sistema (1.2) está sujeito a variação do parâmetro $\lambda$, bifurcações locais podem ocorrer na fronteira da região de estabilidade e consequentemente pontos de equilíbrio 
não hiperbólicos podem aparecer na fronteira da região de estabilidade violando assim a hipótese $(A 1)$. Como queremos entender o comportamento da região de estabilidade e de sua fronteira para propor estimativas da região de estabilidade quando ocorrem estas bifurcações, buscaremos responder duas perguntas importantes: $(i)$ pode-se ainda obter uma caracterização da fronteira da região de estabilidade em termos das variedades estáveis dos pontos de equilíbrio que estão na sua fronteira, incluindo o ponto de equilíbrio não hiperbólico? (ii) como a região de estabilidade e sua fronteira se comportam sob pequenas variações do parâmetro $\lambda$ na ocorrência de uma bifurcação local na fronteira? Neste trabalho, o objetivo é responder a estas perguntas para um caso específico de bifurcação local, que é a bifurcação sela-nó do tipo zero, e propor um algoritmo conceitual para obter estimativas da região de estabilidade perturbada, via conjunto de nível de uma dada função energia, na vizinhança de um parâmetro de bifurcação sela-nó do tipo zero.

\subsection{Contribuições}

Neste trabalho, supondo a existência de um ponto de equilíbrio não hiperbólico selanó do tipo zero na fronteira da região de estabilidade, apresentaremos uma caracterização completa da fronteira em termos das variedades estáveis dos pontos de equilíbrio que estão na fronteira, incluindo o ponto de equilíbrio não hiperbólico sela-nó do tipo zero. Mostraremos também que, mesmo com a presença de um ponto de equilíbrio sela-nó do tipo zero na fronteira da região de estabilidade, os pontos de equilíbrio hiperbólicos na fronteira da região de estabilidade persistem sob a influência de pequenas variações dos parâmetros. Resultados que descrevem o comportamento da fronteira da região de estabilidade na vizinhanças de um ponto de equilíbrio sela-nó do tipo zero serão apresentados. Além disso, obteremos uma caracterização global da fronteira da região de estabilidade nas vizinhanças de um parâmetro de bifurcação sela-nó do tipo zero. E por fim, ofereceremos uma estimativa da região de estabilidade, via conjunto de nível de uma dada função energia, na presença de um ponto de equilíbrio sela-nó do tipo zero na fronteira da região de estabilidade e exibiremos resultados que permitem entender o comportamento desta estimativa na vizinhança de um parâmetro de bifurcação sela-nó do tipo zero.

\subsection{Organização do texto}

Os próximos capítulos deste texto estão organizados da seguinte forma:

- No Capítulo 2, uma revisão de alguns conceitos e resultados na área de topologia e equações diferencias ordinárias é apresentada.

- No Capítulo 3, o conceito de conjunto atrativo e atrator é introduzido e algumas discussões desses conceitos são apresentadas. Além disso, exibimos uma caracterização topológica da região de estabilidade e de sua fonteira. 
- No Capítulo 4, uma breve revisão sobre a teoria existente da caracterização dinâmica da fronteira da região de estabilidade é apresentada.

- No Capítulo 5, o conceito de ponto de equilíbrio sela-nó do tipo zero é apresentado, exibiremos uma versão do $\lambda$-Lema para ponto de equilíbrio sela-nó do tipo zero, forneceremos condições necessárias e suficientes para que um ponto de equilíbrio sela-nó do tipo zero pertença à fronteira da região de estabilidade de um ponto de equilíbrio assintoticamente estável e desenvolvemos resultados que permitem caracterizar a fronteira da região de estabilidade em termos das variedades estáveis dos pontos de equilíbrio que pertencem a fronteira, incluindo o ponto de equilíbrio sela-nó do tipo zero. Além disso, definiremos o conceito de região de estabilidade fraca e exploraremos algumas de suas propriedades.

- No Capítulo 6, discute-se a persistência da região de estabilidade e da caracterização de sua fronteira sob a infuência de pequenas variações do parâmetro.

- No Capítulo 7, uma breve revisão sobre a teoria de bifurcação para uma família de equações diferenciais dependendo de um parâmetro escalar é apresentada.

- No Capítulo 8, mostraremos o comportamento da fronteira da região de estabilidade na vizinhança de um ponto de equilíbrio sela-nó do tipo zero. Com a informação deste comportamento local, verificaremos que mudanças drásticas podem ocorrer na região de estabilidade e na fronteira da região de estabilidade sob a infuência de pequenas variações do parâmetro. Exibiremos também uma caracterização global da fronteira da região de estabilidade para valores de parâmetros próximos a um parâmetro de bifurcação sela-nó do tipo zero.

- No Capítulo 9, a definição de função energia e uma revisão dos resultados relacionados a estimativas da região de estabilidade via conjunto de nível de uma dada função energia são apresentadas. Apresentaremos também estimativas da região de estabilidade via conjunto de nível de uma dada função energia na presença de um ponto de equilíbrio sela-nó do tipo zero na fronteira. Exibiremos também resultados que permitem entender o comportamento destas estimativas sob a influência das variações do parâmetro, incluindo variações próximas a um parâmetro de bifurcação sela-nó do tipo zero. 


\section{PRELIMINARES}

Este capítulo tem como finalidade rever alguns conceitos e resultados na área de topologia e equações diferencias ordinárias que serão usados ao longo do texto. Iniciaremos com uma discussão sobre a topologia do espaço euclidiano. Posteriormente, discutiremos alguns tópicos da teoria de equações diferencias ordinárias.

Ao leitor familiarizado com os conceitos de topologia e equações diferencias ordinárias a leitura deste capítulo pode ser dispensada, retornando ao mesmo conforme a necessidade.

\subsection{Espaço Euclidiano}

Nesta seção, exibiremos alguns resultados sobre a topologia do espaço euclidiano que serão explorados ao decorrer do trabalho. Para maiores esclarecimentos/detalhes dos assuntos aqui tratados ver (LIMA, 2006) e (MUNKRES, 2000).

\subsubsection{Norma}

Seja $n$ um número natural. O espaço euclidiano $n$-dimensional é o produto de $n$ fatores iguais a $\mathbb{R}$ :

$$
\mathbb{R}^{n}=\mathbb{R} \times \mathbb{R} \times \ldots \times \mathbb{R}
$$

Os pontos de $\mathbb{R}^{n}$ são todas as $n$-listas $x=\left(x_{1}, \ldots, x_{n}\right)$ cujas coordenadas $x_{1}, \ldots, x_{n}$ são números reais.

É conhecido da álgebra linear que $\mathbb{R}^{n}$ com as operações usuais de soma e multiplicação tem estrutura de espaço vetorial de dimensão $n$ sobre o corpo dos reais.

Um produto interno num espaço vetorial real $E$ é uma aplicação $\langle\rangle:, E \times E \longrightarrow \mathbb{R}$ que faz corresponder a cada par de vetores $x, y \in E$ um número real, indicado por $\langle x, y\rangle$, de tal modo que, para quaisquer $x, z, y \in E$ e $\alpha \in \mathbb{R}$, valham as seguintes propriedades:

(P1.) $\langle x, y\rangle=\langle y, x\rangle ;$ (Comutatividade)

(P2.) $\langle x+z, y\rangle=\langle x, y\rangle+\langle z, y\rangle ;($ Superposição)

(P3.) $\langle\alpha x, y\rangle=\alpha \cdot\langle x, y\rangle=\langle x, \alpha y\rangle$; (Homogeneidade)

(P4.) $x \neq 0 \Longrightarrow\langle x, x\rangle>0$. (Positividade) 
Um exemplo de produto interno em $\mathbb{R}^{n}$, e o mais importante, é o produto interno canônico, o qual é dado por

$$
\langle x, y\rangle=x_{1} \cdot y_{1}+\ldots+x_{n} \cdot y_{n}
$$

onde $x=\left(x_{1}, \ldots, x_{n}\right)$ e $y=\left(y_{1}, \ldots, y_{n}\right)$.

Dado $x \in \mathbb{R}^{n}$, escreveremos $\|x\|=\sqrt{\langle x, x\rangle}$, onde $\langle$,$\rangle é o produto interno canônico.$ Assim,

$$
\|x\|=\sqrt{x_{1}^{2}+\ldots+x_{n}^{2}}
$$

O número $\|x\|$ chama-se norma euclidiana. A norma euclidiana goza das seguintes propriedades, onde $x, y \in \mathbb{R}^{n}, \alpha \in \mathbb{R}$ e $|\alpha|$ indica o valor absoluto do número real $\alpha$ :

(N1.) $\|x+y\| \leqslant\|x\|+\|y\| ;$ (Desigualdade triangular)

(N2.) $\|\alpha . x\|=|\alpha| \cdot\|x\| ;$ (Homogeneidade)

(N3.) $x \neq 0 \Longrightarrow\|x\|>0$. (Positividade)

De um modo geral, uma norma num espaço vetorial $E$ é qualquer função real \|\| : $E \longrightarrow \mathbb{R}$ que cumpra as condições (N1), (N2) e (N3) acima. Há uma infinidade de normas que se podem considerar no espaço euclidiano . Quando não dissermos explicitamente qual a norma que estamos considerando em $\mathbb{R}^{n}$, fica subentendido que se trata da euclidiana.

Uma norma num espaço vetorial $E$ dá origem a uma noção de distância em $E$. Dados $x, y \in E$, a distância de $x$ a $y$ é definida por

$$
d(x, y)=\|x-y\|
$$

Verifica-se facilmente que a distância goza das seguintes propriedades, para $x, y, z \in E$ quaisquer:

(D1.) $d(x, y) \leqslant d(x, y)+d(y, z) ;$ (Desigualdade triangular)

(D2.) $d(x, y)=d(y, x)$; (Comutatividade)

(D3.) $x \neq y \Longrightarrow d(x, y)>0$. (Positividade)

A primeira dessas propriedades é chamada desigualdade triangular. Neste texto, estaremos considerando no espaço euclidiano $\mathbb{R}^{n}$ a distância proveniente da norma euclidiana, salvo menção contrária.

\subsubsection{Conjuntos Abertos}

Antes de definirmos o que é um conjunto aberto em $\mathbb{R}^{n}$, introduziremos o conceito de bolas.

A bola aberta de centro num ponto $a \in \mathbb{R}^{n}$ e raio $r>0$ é o conjunto dos pontos $x \in \mathbb{R}^{n}$ cuja distância ao ponto $a$ é menor que $r$. Usaremos a notação $B(a ; r)$ para indicar esse 
conjunto. Assim,:

$$
B(a ; r)=\left\{x \in \mathbb{R}^{n} ;\|x-a\|<r\right\} .
$$

Analogamente, definiremos a bola fechada $B[a ; r]$ e a esfera $S[a ; r]$, ambas com centro $a$ e raio $r$, por:

$$
B[a ; r]=\left\{x \in \mathbb{R}^{n} ;\|x-a\| \leqslant r\right\} \text { e } S[a ; r]=\left\{x \in \mathbb{R}^{n} ;\|x-a\|=r\right\} .
$$

Segue-se que $B[a ; r]=B(a ; r) \cup S[a ; r]$.

Seja $X$ um subconjunto do espaço euclidiano $\mathbb{R}^{n}$. Um ponto $a \in X$ chama-se um ponto interior a $X$ quando é centro de alguma bola aberta contida em $X$, ou seja, quando existe $r>0$ tal que $\|x-a\|<r \Longrightarrow x \in X$. O interior de $X$ é o conjunto int $X$ formado pelos pontos interiores a $X$. Quando $x \in$ int $V$, dizemos que o conjunto $V$ é uma vizinhança do ponto $x$.

Um conjunto $X \subset \mathbb{R}^{n}$ chama-se aberto quando todos os seus pontos são interiores, isto é, quando para cada $x \in X$ existe $r>0$ tal que $B(x ; r) \subset X$. Assim, $X$ é aberto $\Longleftrightarrow$ int $X=X$.

Uma bola aberta $B(a ; r) \subset \mathbb{R}^{n}$ é um exemplo de conjunto aberto em $\mathbb{R}^{n}$, assim como o conjunto $X=\mathbb{R}^{n}-B[a ; r]$ também é aberto em $\mathbb{R}^{n}$. Uma outra informação sobre conjuntos abertos é que para todo conjunto $X \subset \mathbb{R}^{n}$, int $X$ é um conjunto aberto.

Teorema 2.1.1. (LIMA, 2006) Os conjuntos abertos do espaço euclidiano $\mathbb{R}^{n}$ gozam das seguintes propriedades:

1) O conjunto vazio $\emptyset$ e o espaço $\mathbb{R}^{n}$ são abertos;

2) $A$ interseção $A=A_{1} \cap \ldots \cap A_{k}$ de um número finito de conjuntos abertos $A_{1}, \ldots, A_{k}$ é um conjunto aberto;

3) A reunião $A=\cup_{\lambda \in L} A_{\lambda}$ de uma família qualquer $\left(A_{\lambda}\right)_{\lambda \in L}$ de conjuntos abertos $A_{\lambda}$ é um conjunto aberto.

Fixemos um conjunto $X \subset \mathbb{R}^{n}$. Um subconjunto $A \subset X$ diz-se aberto em $X$ quando, para cada $a \in A$ existe $r>0$ tal que $B(a ; r) \cap X \subset A$. Em outras palavras, para cada $a \in A$ existe $r>0$ tal que os pontos $x$, pertencentes a $X$, que cumprem a condição $\|x-a\|<r$ estão em $A$;

Por exemplo, $A=[-1,1)$ é aberto em $X=[-1,1]$, mas não é aberto em $\mathbb{R}$.

Um conjunto $A \subset X$ é aberto em $X \subset \mathbb{R}^{n}$ se, e somente se, existe um aberto $B \subset \mathbb{R}^{n}$ tal que $A=X \cap B$.

\subsubsection{Conjuntos Fechados}

Um ponto $a \in \mathbb{R}^{n}$ diz-se aderente a um conjunto $X \subset \mathbb{R}^{n}$ quando, para todo $r>0$, a bola aberta $B(a ; r)$ contém algum ponto de $X$.

A fim de que o ponto $a$ seja aderente ao conjunto $X$, é necessário e suficiente que o ponto $a$ seja limite de uma sequência de pontos desse conjunto. 
O conjunto dos pontos aderentes a $X$ chama-se fecho de $X$ e é indicado com a notação $\bar{X}$.

Pelo que vimos acima, a fim de que um ponto $b \in \mathbb{R}^{n}$ não pertença ao fecho de $X$, é necessário e suficiente que exista uma bola aberta de centro $b$ que não contenha pontos de $X$. Em outros termos: $b \in \mathbb{R}^{n}-\bar{X} \Longleftrightarrow \exists r>0 ; B(b ; r) \cap X=\emptyset$.

Como toda bola aberta é um conjunto aberto e todo aberto que contém um ponto contém também uma bola aberta com centro nesse ponto, as condições acima podem ser reformuladas com abertos, em vez de bolas:

1) Tem-se $a \in \bar{X}$ se, e somente se, todo aberto que contém $a$ intersecta o conjunto $X$. (Isto é, se $A$ é aberto e $a \in A \Longrightarrow$ então $A \cap X \neq \emptyset$.)

2) Tem-se $b \notin \bar{X}$ se, e somente se, existe um aberto contendo $b$ disjunto de $X$. (Isto é, existe $A$ aberto com $b \in A$ e $A \cap X=\emptyset$.)

Como exemplo, o fecho de uma bola aberta $B(a ; r)$ é a bola fechada $B[a ; r]$. Se $X=\mathbb{Q}^{n}$ é o conjunto dos pontos de $\mathbb{R}^{n}$ cujas coordenadas são números racionais, então $\bar{X}=\mathbb{R}^{n}$.

Um conjunto $X \subset \mathbb{R}^{n}$ chama-se fechado quando contém todos seus pontos aderentes, isto é, quando $X=\bar{X}$.

Por exemplo, uma bola fechada $B[a ; r]$ é um subconjunto fechado de $\mathbb{R}^{n}$, assim como também é fechado a esfera $S[a ; r]$.

Se $x_{k} \rightarrow a$ quando $k \rightarrow+\infty, x_{k} \in X$ para todo $k \in \mathbb{N}$ e $A$ é um conjunto fechado, então $a \in X$.

Dados um conjunto $X$ e um ponto $a \in \mathbb{R}^{n}$, há três possibilidades que se excluem mutuamente: ou $a \in$ int $X$, ou $a \in$ int $\left(\mathbb{R}^{n}-X\right)$ ou então toda bola aberta de centro $a$ contém pontos de $X$ e pontos do complementar de $X$. Os pontos com esta última propriedade constituem $\partial X$, que chamaremos a fronteira de $X$. Em outras palavras, $\partial X=\bar{X} \cap \overline{\mathbb{R}^{n}-X}$.

Os próximos dois teoremas, cujas demonstrações foram tiradas de (LIMA, 2006), permitem concluir que a fronteira de um conjunto $X \subset \mathbb{R}^{n}$ é um conjunto fechado.

Teorema 2.1.2. (LIMA, 2006) Para todo $X \subset \mathbb{R}^{n}$, o complementar do fecho de $X$ é um conjunto aberto.

Demonstração. Considere $A=\mathbb{R}^{n}-\bar{X}$. Para todo $a \in A$, existe $r>0$ tal que $B(a ; r) \cap X=$ $\emptyset$. Afirmamos que $B(a ; r) \subset A$. De fato, se $z \in B(a ; r)$, então $B(a ; r)$ é um aberto contendo $z$ e disjunto de $X, \log o z \in \mathbb{R}^{n}-\bar{X}=A$.

Teorema 2.1.3. (LIMA, 2006) Um conjunto é fechado no $\mathbb{R}^{n}$ se, e somente se, seu complementar é aberto no $\mathbb{R}^{n}$.

Demonstração. $(\Longrightarrow)$ Se $X \subset \mathbb{R}^{n}$ é fechado, temos que $X=\bar{X}$, em particular, $\mathbb{R}^{n}-X=$ $\mathbb{R}^{n}-\bar{X}$ e pelo teorema anterior podemos afirmar que o complementar de $X$ é aberto.

$(\Longleftarrow)$ Se $X \subset \mathbb{R}^{n}$ é tal que $A=\mathbb{R}^{n}-X$ é um conjunto aberto então $z \notin X$ implica em $z \in A$. Como $A$ é aberto por hipótese existe $r>0$ tal que $B(z ; r) \subset A$, ou seja, $B(z ; r) \cap X=\emptyset$, 
consequentemente $z$ não é aderente a $X$. Assim, todo ponto aderente a $X$ deve pertencer a $X$ e portanto $X$ é fechado.

Os dois teoremas anteriores garantem que o fecho de qualquer conjunto é um conjunto fechado. Como a fronteira é a interseção de dois conjuntos fechados, por consequência, a fronteira de todo conjunto $X \subset \mathbb{R}^{n}$ é um conjunto fechado. O seguinte resultado é uma consequência imediata do Teorema 2.1.1 e do Teorema 2.1.3.

Teorema 2.1.4. (LIMA, 2006) Os conjuntos fechados do espaço euclidiano $\mathbb{R}^{n}$ gozam das seguintes propriedades:

1) O conjunto vazio $\emptyset$ e o espaço $\mathbb{R}^{n}$ são fechados;

2) A união $F=F_{1} \cup \ldots \cup F_{k}$ de um número finito de conjuntos fechados $F_{1}, \ldots, F_{k}$ é um conjunto fechado;

3) A interseção $F=\cap_{\lambda \in L} F_{\lambda}$ de uma família qualquer $\left(F_{\lambda}\right)_{\lambda \in L}$ de conjuntos fechados $F_{\lambda}$ é um conjunto fechado.

O conjunto formado pela união infinita de conjuntos fechados pode não ser fechado. De fato, para cada ponto $x \in \mathbb{Q} \subset \mathbb{R}$, o conjunto $\{x\}$ é fechado em $\mathbb{R}$. O conjunto $\mathbb{Q}$ é união dos seus pontos, isto é, $\mathbb{Q}=\cup_{x \in \mathbb{Q}}\{x\}$ e o mesmo não é fechado em $\mathbb{R}$ visto que $\overline{\mathbb{Q}}=\mathbb{R}$.

Fixemos um conjunto $X \subset \mathbb{R}^{n}$. Um subconjunto $F \subset X$ diz-se fechado em $X$ quando se tem $F=X \cap G$ onde $G$ é um conjunto fechado em $\mathbb{R}^{n}$.

Se $X \subset \mathbb{R}^{n}$ é fechado, então um subconjunto $F \subset X$ é fechado em $X$ se, e somente se, é fechado em $\mathbb{R}^{n}$.

Dados $Y \subset X \subset \mathbb{R}^{n}$, podemos também definir o fecho de $Y$ relativamente a $X$ como sendo o conjunto $\bar{Y} \cap X$, dos pontos aderentes a $Y$ que pertencem ao conjunto $X$.

Sejam $Y \subset X \subset \mathbb{R}^{n}$. Dizemos que $Y$ é denso em $X$ quando $\bar{Y} \cap X=X$, ou seja, $X \subseteq \bar{Y}$.

\subsubsection{Conjuntos Conexos}

Uma cisão de um conjunto $X \subset \mathbb{R}^{n}$ é uma decomposição $X=A \cup B$, onde $A \cap B=\emptyset \mathrm{e}$ os conjuntos $A, B$ são ambos abertos em $X$. As condições $X=A \cup B$ e $A \cap B=\emptyset$ equivalem a dizer que $A=X-B$ e $B=X-A$. Por conseguinte, numa cisão $X=A \cup B$, os conjuntos $A, B$ são abertos e fechados em $X$.

Todo conjunto $X \subset \mathbb{R}^{n}$ admite pelo menos a cisão trivial $X=X \cup \emptyset$. Um exemplo de cisão não trivial é $\mathbb{R}-\{0\}=(-\infty, 0) \cup(0,+\infty)$.

Um conjunto $X \subset \mathbb{R}^{n}$ chama-se conexo quando não admite outra cisão além da trivial. Assim, quando $X$ é conexo, $X=A \cup B, \operatorname{com} A, B$ disjuntos e abertos em $X$, implica $A=\emptyset$ ou $B=\emptyset$.

Quando existir uma cisão não-trivial $X=A \cup B$, diremos que $X$ é desconexo.

A imagem de um conjunto conexo por uma aplicação contínua é um conjunto conexo. Um subconjunto $X \subset \mathbb{R}$ é conexo se, e somente se, é um intervalo. Uma outra propriedade 
importante é que a união de uma família de conjuntos conexos com um ponto em comum é um conjunto conexo.

A definição de conjunto conexo proposta acima é a expressão matemática da ideia de conjunto formado por um só pedaço. Outra maneira de exprimir a conexidade é dizer que se pode passar de qualquer um dos seus pontos para outro por um movimento contínuo, sem sair do conjunto. Isto nos leva à noção de espaço conexo por caminhos.

Sejam $x, y \in \mathbb{R}^{n}$. O segmento de reta de extremos $x, y$ é o conjunto

$$
[x, y]=\{(1-t) x+t y ; 0 \leqslant t \leqslant 1\} .
$$

Um subconjunto $X \subset \mathbb{R}^{n}$ diz-se convexo quando contém qualquer segmento de reta cujos extremos pertençam a $X$, ou seja: $x, y \in X \Longrightarrow[x, y] \subset X$.

Todo espaço vetorial $E \subset \mathbb{R}^{n}$ é convexo, assim como toda bola $B \subset \mathbb{R}^{n}$ é convexa.

Um caminho num conjunto $X \subset \mathbb{R}^{n}$ é uma aplicação contínua $f: I \longrightarrow X$, definida num intervalo $I$.

Diremos que os pontos $a, b \in X$ podem ser ligados por um caminho em $X$ quando existe uma caminho $f: I \longrightarrow X$ tal que $a, b \in f(I)$.

Um conjunto $X \subset \mathbb{R}^{n}$ diz-se conexo por caminhos quando dois pontos quaisquer $a, b \in$ $X$ podem ser ligados por um caminho em $X$.

Por exemplo, se $X \subset \mathbb{R}^{n}$ é convexo, dois pontos quaisquer $a, b \in X$ podem ser ligados por um caminho em $X$, a saber o caminho retilíneo $f(t)=(1-t) a+t b$ para todo $t \in[0,1]$. Portanto, conjuntos convexos são casos particulares de conjuntos conexos por caminhos, entretanto, existem conjuntos conexos por caminhos que não são convexos. O conjunto $\mathbb{R}^{n}-\{0\}$, com $n>1$, por exemplo, é conexo por caminho mas não é convexo.

Se $f, g:[0,1] \longrightarrow X$ são caminhos em $X, \operatorname{com} f(1)=g(0)$, então definimos o caminho justaposto $h=f \underline{\vee} g:[0,1] \longrightarrow X$ fazendo $h(t)=f(2 t)$ se $0 \leqslant t \leqslant \frac{1}{2}$ e $h(t)=g(2 t-1)$ se $\frac{1}{2} \leqslant t \leqslant 1$. Estas duas expressões definem o mesmo valor de $h\left(\frac{1}{2}\right)$. Como $\left.f\right|_{\left[0, \frac{1}{2}\right]}$ e $\left.g\right|_{\left[\frac{1}{2}, 1\right]}$ são contínuas, segue-se que $h$ é contínua.

Sejam $a, b, c$ pontos do conjunto $X \subset \mathbb{R}^{n}$. Se $a, b$ podem ser ligados por um caminho em $X$ e $b, c$ também podem ser ligados por um caminho em $X$, então existe um caminho em $X$ ligando $a$ a $c$. Basta tomar caminhos $f, g:[0,1] \longrightarrow X \operatorname{com} f(0)=a, f(1)=b$, $g(0)=b, g(1)=c$ e por $h=f \underline{\vee} g$. Então $h(0)=a$ e $h(1)=c$. Essa propriedade de caminho descrita acima é costumeiramente chamada de transitiva.

Teorema 2.1.5. (LIMA, 2006) Se um conjunto $X \subset \mathbb{R}^{n}$ é conexo por caminho, então $X$ é conexo.

Demonstração. Suponhamos por contrapositiva que $X=A \cup B$ fosse uma cisão não-trivial de $X$. Tomemos $a \in A$ e $b \in B$. Por hipótese, existiria um caminho $f:[0,1] \longrightarrow X$ tal que $f(0)=a, f(1)=b$. Então $[0,1]=f^{-1}(A) \cup f^{-1}(B)$ seria uma cisão de $[0,1]$, com $0 \in f^{-1}(A)$ e $1 \in f^{-1}(B)$, o que é um absurdo, pois o intervalo [0,1] é conexo. 
A recíproca do Teorema 2.1.5 não é verdadeira. Como exemplo considere $X \subset \mathbb{R}^{2}$ o gráfico da função $f:[0,+\infty) \longrightarrow \mathbb{R}$, dada por $f(x)=\cos \left(\frac{1}{x}\right)$ se $x>0$ e $f(0)=0$. O conjunto $X$ é conexo mas não é conexo por caminho, para maiores detalhes ver (LIMA, 2006). Esse exemplo junto com o Teorema 2.1.5 nos permite afirmar que o conceito de conexidade por caminho é mais forte do que o conceito de conexidade definida por meio de cisão.

\subsubsection{Conjuntos Contráteis}

Sejam os conjuntos $X, Y \subset \mathbb{R}^{n}$ e $f, g: X \longrightarrow Y$ aplicações contínuas, dizemos que $f$ é homotópica à $g$ quando existe uma aplicação contínua $H: X \times[0,1] \longrightarrow Y$ tal que $H(x, 0)=f(x)$ e $H(x, 1)=g(x)$ para todo $x \in X$.

A aplicação $H$ é uma homotopia entre $f$ e $g$ e, é denotada por $f \simeq g$.

Como exemplo, se $X, Y \subset \mathbb{R}^{2}$ e $Y$ é convexo, então todas as aplicações contínuas de $X$ em $Y$ são homotópicas, pois dados $f, g: X \longrightarrow Y$ basta definirmos $H: X \times[0,1] \longrightarrow Y$ por $H(x, t)=t f(x)+(1-t) g(x)$.

A relação de homotopia é compatível com composição de funções, ou seja, dadas as aplicações contínuas $f, f^{\prime}: X \longrightarrow Y$ e $g, g^{\prime}: Y \longrightarrow Z$ tais que $f \simeq f^{\prime}$ e $g \simeq g^{\prime}$ então $g \circ f \simeq g^{\prime} \circ f^{\prime}$.

Um conjunto $X \subset \mathbb{R}^{n}$ é contrátil se a aplicação identidade $i d_{X}: X \longrightarrow X$ é homotópica a uma aplicação constante.

Todo subconjunto convexo de $\mathbb{R}^{n}$ é contrátil. Além disso, todo conjunto contrátil $X$ é conexo por caminho, mas a recíproca não é verdadeira já que $\mathbb{R}^{2}-\{0\}$ é conexo por caminho e não é contrátil.

\subsubsection{Espaços Normais}

Seja $X$ um espaço topológico. Dizemos que $X$ é um espaço normal se para cada par $A, B$ de conjuntos fechados disjuntos de $X$, existem abertos disjuntos contendo $A$ e $B$, respectivamente.

O resultado a seguir nos garante que o $\mathbb{R}^{n}$ é um espaço normal.

Teorema 2.1.6. (MUNKRES, 2000) $\mathbb{R}^{n}$ é um espaço normal.

Demonstração. Sejam $x_{0} \in \mathbb{R}^{n}, r>0$ número real positivo e $B\left(x_{0}, r\right)=\left\{x \in \mathbb{R}^{n}: d\left(x, x_{0}\right)<\right.$ $r$ \}. Sejam $A$ e $B$ dois subconjuntos fechados disjuntos de $\mathbb{R}^{n}$. Note que, $\mathbb{R}^{n}-A$ e $\mathbb{R}^{n}-B$ são subconjuntos abertos de $\mathbb{R}^{n}$. Assim, para cada $a \in A$ podemos afirmar que $a \in \mathbb{R}^{n}-B$ que é aberto, logo existe $\varepsilon_{a}>0$ tal que $B\left(a, \varepsilon_{a}\right) \subset \mathbb{R}^{n}-B$, isto é, $B\left(a, \varepsilon_{a}\right)$ não intercepta $B$. Similarmente, para cada $b \in B$ existe $\varepsilon_{b}>0$ tal que $B\left(b, \varepsilon_{b}\right)$ não intercepta $A$. Defina

$$
U=\cup_{a \in A} B\left(a, \frac{\varepsilon_{a}}{2}\right) \text { e } V=\cup_{b \in B} B\left(b, \frac{\varepsilon_{b}}{2}\right)
$$


Então $U$ e $V$ são subconjuntos abertos contendo $A$ e $B$, respectivamente. Afirmamos que $U$ e $V$ são disjuntos. De fato; se $z \in U \cap V$ então

$$
z \in B\left(a, \frac{\varepsilon_{a}}{2}\right) \cap B\left(b, \frac{\varepsilon_{b}}{2}\right)
$$

para algum $a \in A$ e para algum $b \in B$. Usando a desigualdade triangular podemos afirmar que $d(a, b) \leqslant d(a, z)+d(z, b)<\frac{\varepsilon_{a}}{2}+\frac{\varepsilon_{b}}{2}$. Se $\varepsilon_{a} \leqslant \varepsilon_{b}$ então $d(a, b)<\frac{\varepsilon_{a}}{2}+\frac{\varepsilon_{b}}{2} \leqslant \frac{\varepsilon_{b}}{2}+\frac{\varepsilon_{b}}{2}=\varepsilon_{b}$, ou seja, $a \in B\left(b, \varepsilon_{b}\right)$. Se $\varepsilon_{b} \leqslant \varepsilon_{a}$ então $d(a, b)<\frac{\varepsilon_{a}}{2}+\frac{\varepsilon_{b}}{2} \leqslant \frac{\varepsilon_{a}}{2}+\frac{\varepsilon_{a}}{2}=\varepsilon_{a}$, ou seja, $b \in$ $B\left(a, \varepsilon_{a}\right)$. Mas nenhuma dessas situações podem ocorrer, o que demonstra o teorema.

O teorema anterior pode ser estendido para todos espaços topológicos que são metrizáveis, assim existe uma coleção grande de espaços topológicos normais.

\subsubsection{Distância entre dois conjuntos}

Sejam $S, T \subset \mathbb{R}^{n}$ conjuntos não vazios. Definiremos a distância $d(S, T)$ entre $S$ e $T$ por

$$
d(S, T)=\inf \{\|x-y\| ; x \in S, y \in T\} .
$$

A distância entre dois conjuntos satisfaz as seguintes propriedades:

1) $d(S, T)=d(T, S)$;

2) $S \cap T \neq \emptyset \Longrightarrow d(S, T)=0$;

3) $S_{1} \subset S_{2}, T_{1} \subset T_{2} \Longrightarrow d\left(S_{2}, T_{2}\right) \leqslant d\left(S_{1}, T_{1}\right)$;

4) $d(S, T) \leqslant\|x-y\|$ para quaisquer $x \in S$ e $y \in T$;

5) Dado arbitrariamente $\varepsilon>0$, existem $x \in S$ e $y \in T$ tais que $\|x-y\|<d(S, T)+\varepsilon$.

Um caso particular importante da distância entre dois conjuntos ocorre quando um deles se reduz a um ponto.

Dado $x \in \mathbb{R}^{n}$ e um conjunto não vazio $T \subset \mathbb{R}^{n}$, temos:

$$
d(x, T)=\inf \{\|x-y\| ; y \in T\}
$$

Novamente, valem as cinco propriedades descritas anteriormente.

Usando a propriedade que o ínfimo de um conjunto de números não-negativos é igual a zero se, e somente se, tal conjunto contém números arbitrariamente pequenos podemos afirmar que $d(x, T)=0$ se, e somente se, dado qualquer $\varepsilon>0$, existe $y \in T$ tal que $d(x, y)<\varepsilon$. Em outras palavras,

$$
d(x, T)=0 \Longleftrightarrow x \in \bar{T} .
$$

Em particular, se $F \subset \mathbb{R}^{n}$ é um conjunto fechado, vale $d(x, F)=0 \Longleftrightarrow x \in F$.

Como $\partial T=\bar{T} \cap \overline{\mathbb{R}^{n}-T}$, vemos que $x \in \partial T$ se, e somente se, $d(x, T)=d\left(x, \mathbb{R}^{n}-T\right)=$ 0 . 
Um outro resultado importante que envolve distância é que a função $f: \mathbb{R}^{n} \longrightarrow \mathbb{R}$, definida por $f(x)=d(x, T)$, é uniformemente contínua.

\subsection{Equações Diferenciais Ordinárias}

Neste trabalho, estamos interessados em estudar sistemas dinâmicos não lineares modelados por equações diferenciais ordinárias (EDO) autônomas não lineares do tipo:

$$
\dot{x}=f(x)
$$

onde $f: \mathbb{R}^{n} \longrightarrow \mathbb{R}^{n}$ é um campo vetorial de classe $C^{1}$. Começaremos a seção apresentando um resultado de existência e unicidade de equações diferenciais ordinárias autônomas. Na Seção 2.2.2, introduziremos o conceito de conjunto invariante. Nas Seções 2.2.3, 2.2.4 e 2.2.5 exploraremos estabilidade local e comportamento assintótico de soluções de equações diferenciais ordinárias autônomas. Para complemento ou maiores detalhes das definições e resultados expostos nesta seção ver (SOTOMAYOR, 1979), (HALE, 1969), (SMALE; HIRSCH, 1974), (WIGGINS, 2003), (PERKO, 1991), (ALBERTO, 2006), (SMALE, 1967), (HIRSCH; PUGH; SHUB, 1970), (GUCKENHEIMER; HOLMES, 1983) e (SHUB, 1987).

\subsubsection{Teoria Geral}

Sejam $f: \mathbb{R}^{n} \longrightarrow \mathbb{R}^{n}$ um campo vetorial de classe $C^{1}$ e $I$ um intervalo não degenerado da reta, isto é, um subconjunto conexo de $\mathbb{R}$ não reduzido a um ponto.

Definição 2.2.1. Uma função diferenciável $\varphi: I \longrightarrow \mathbb{R}^{n}$ é uma solução da equação diferencial ordinária

$$
\dot{x}=f(x)
$$

no intervalo I se

$$
\frac{d \varphi}{d t}(t)=f(\varphi(t)) \text { para todo } t \in I
$$

Se uma condição inicial é conhecida, o problema de encontrar a solução $\varphi(t)$, satisfazendo essa condição inicial é conhecido como problema de valor inicial (PVI):

$$
\dot{x}=f(x) ; x(0)=x_{0}
$$

O teorema de existência e unicidade, demonstrado em (SOTOMAYOR, 1979) e apresentado a seguir, estabelece condições suficientes sobre o campo vetorial $f$ para garantir a existência e a unicidade das soluções.

Teorema 2.2.1. (SOTOMAYOR, 1979) Sejam $f: \mathbb{R}^{n} \longrightarrow \mathbb{R}^{n}$ um campo vetorial de classe 
$C^{1}$ e $x_{0} \in \mathbb{R}^{n}$. Então existe um $\alpha>0$ tal que o problema de valor inicial

$$
\dot{x}=f(x) ; x(0)=x_{0}
$$

tem uma única solução $\varphi(t)$ no intervalo $[-\alpha, \alpha]$.

Supondo ainda que as hipóteses do teorema anterior estejam satisfeitas, então, para cada $x_{0} \in \mathbb{R}^{n}$, existe uma única solução maximal $\varphi\left(., x_{0}\right)$ de

$$
\dot{x}=f(x) ; x(0)=x_{0}
$$

definida num intervalo maximal de existência $\left(\omega_{-}\left(x_{0}\right), \omega_{+}\left(x_{0}\right)\right)$.

Um outro aspecto importante da teoria de equações diferenciais ordinárias é a continuidade das soluções com relação às condições inicias apresentada no seguinte teorema:

Teorema 2.2.2. (SOTOMAYOR, 1979) Seja $f: \mathbb{R}^{n} \longrightarrow \mathbb{R}^{n}$ um campo vetorial de classe $C^{1}$. Considere $\varphi\left(t, x_{0}\right)$ a solução do PVI

$$
\dot{x}=f(x) ; x(0)=x_{0}
$$

definida no seu intervalo maximal de existência. Então

$$
D=\left\{\left(t, x_{0}\right): x_{0} \in \mathbb{R}^{n}, t \in\left(\omega_{-}\left(x_{0}\right), \omega_{+}\left(x_{0}\right)\right)\right\}
$$

é aberto em $\mathbb{R} \times \mathbb{R}^{n}$ e $\varphi$ é contínua em $D$.

Além da continuidade com relação às condições inicias, a solução de uma EDO autônoma satisfaz as seguintes propriedades:

(1) $\varphi(0, x)=x$ para todo $x \in \mathbb{R}^{n}$.

(2) $\varphi(t+s, x)=\varphi(t, \varphi(s, x))$ para todo $x \in \mathbb{R}^{n}$ e todos $t, s \in \mathbb{R}$ tais que $t+s \in\left(w_{-}(x), w_{+}(x)\right)$ e $t \in\left(w_{-}(\varphi(s, x)), w_{+}(\varphi(s, x))\right)$.

Observação 2.2.1. A aplicação $t \longrightarrow \varphi\left(t, x_{0}\right)$ de $\left(w_{-}\left(x_{0}\right), w_{+}\left(x_{0}\right)\right)$ em $\mathbb{R}^{n}$ define uma curva em $\mathbb{R}^{n}$, passando por $x_{0}$ a qual denomina-se trajetória ou órbita passando por $x_{0}$ e que denotaremos pelo conjunto $\left\{\varphi\left(t, x_{0}\right) ; t \in\left(w_{-}\left(x_{0}\right), w_{+}\left(x_{0}\right)\right)\right\}$. A unicidade das soluções garante que as trajetórias não se interceptam. Dado A um subconjunto de $\mathbb{R}^{n} e$ $t \in \mathbb{R}$, defini-se o conjunto $\varphi(t, A)=\{\varphi(t, x): x \in A\}$.

\subsubsection{Conjuntos Invariantes}

O conceito de invariância que será proposto nesta seção é de fundamental importância para análise de sistemas dinâmicos, isto porque, dado um conjunto $S \subset \mathbb{R}^{n}$, em certas ocasiões, é importante saber quando dada uma condição inicial nesse conjunto a solução que passa por essa condição inicial permanece no respectivo conjunto para todo o tempo, 
para tempos positivos ou negativos, respectivamente. Esses conjuntos com tais propriedades são chamados na literatura de conjuntos invariantes, positivamente invariantes e negativamente invariantes, respectivamente. A fim de formalizar matematicamente esses conceitos, apresentamos as seguintes definições:

Definição 2.2.2. Um conjunto $S \subset \mathbb{R}^{n}$ é invariante com relação ao sistema (2.1) se, para qualquer $x_{0} \in S$ temos $\varphi\left(t, x_{0}\right) \in S$ para todo $t \in \mathbb{R}$.

Definição 2.2.3. Um conjunto $S \subset \mathbb{R}^{n}$ é positivamente (negativamente) invariante com relação ao sistema (2.1) se, para qualquer $x_{0} \in S$ temos $\varphi\left(t, x_{0}\right) \in S$ para todo $t \geqslant 0$ $(t \leqslant 0)$.

Um exemplo trivial de conjunto invariante é a órbita de uma dada condição inicial $x_{0}$ do sistema (2.1). Além disso, sem maiores dificuldades, pode-se verificar que a união de conjuntos invariantes é também um conjunto invariante.

\subsubsection{Comportamento Assintótico}

Dado uma condição inicial do sitema (2.1) podemos nos perguntar o que acontece com a trajetória que passa por $x_{0}$ quando o tempo tende para $+\infty$ ou $-\infty$. Usualmente essas trajetórias se aproximam de equilíbrios, ciclos limites, órbitas quasi-periódicas, órbitas caóticas ou até mesmo união de um certo conjunto de órbitas. Todos esses conjuntos mencionados acima, para onde a trajetória pode se aproximar, são chamados de conjuntos limites cuja definição matemática e algumas de suas propriedades serão exploradas a seguir.

Definição 2.2.4. Um ponto $p \in \mathbb{R}^{n}$ é um ponto $\omega$-limite da solução $\varphi\left(t, x_{0}\right)$ de (2.1) se existir uma sequência $\left\{t_{j}\right\}$, com $t_{j} \longrightarrow+\infty$ quando $j \longrightarrow+\infty$ tal que $\varphi\left(t_{j}, x_{0}\right) \longrightarrow$ $p$ quando $j \longrightarrow+\infty$. O conjunto de todos os pontos $\omega$-limite de $\varphi\left(t, x_{0}\right)$ é chamado conjunto $\omega$-limite da solução $\varphi\left(t, x_{0}\right)$, ou simplesmente, $\omega$-limite de $x_{0}$, e é denotado por $\omega\left(x_{0}\right)$.

Exemplo 2.2.1. Considere um campo vetorial no plano com uma órbita fechada globalmente atrativa $\Omega$, como mostrado na Figura 2.1. Para cada ponto $p \in \Omega$, podemos encontrar uma subsequência $\left\{t_{j}\right\}$ tal que $\varphi\left(t_{j}, x\right), x \in \mathbb{R}^{2}$ se aproxima de p quando $j \rightarrow+\infty$. Portanto, $p \in \Omega$ é um ponto $\omega$-limite e $\Omega$ é o conjunto $\omega$-limite da solução $\varphi(t, x)$.

O comportamento assintótico das soluções quando $t \longrightarrow-\infty$, os chamados conjuntos $\alpha$-limite é dado a seguir.

Definição 2.2.5. Um ponto $p \in \mathbb{R}^{n}$ é um ponto $\alpha$-limite da solução $\varphi\left(t, x_{0}\right)$ de (2.1) se existir uma sequência $\left\{t_{j}\right\}$, com $t_{j} \longrightarrow-\infty$ quando $j \longrightarrow+\infty$ tal que $\varphi\left(t_{j}, x_{0}\right) \longrightarrow$ $p$ quando $j \longrightarrow+\infty$. O conjunto de todos os pontos $\alpha$-limite de $\varphi\left(t, x_{0}\right)$ é chamado conjunto $\alpha$-limite da solução $\varphi\left(t, x_{0}\right)$, ou simplesmente, $\alpha$-limite de $x_{0}$, e é denotado por $\alpha\left(x_{0}\right)$. 


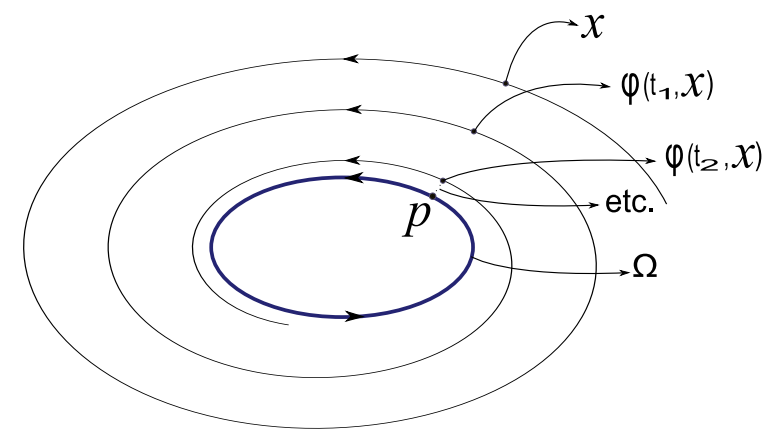

Figura 2.1: $p \in \Omega$ é um ponto $\omega$-limite e $\Omega$ é o conjunto $\omega$-limite da solução $\varphi(t, x)$.

No decorrer da seção, exploraremos alguns teoremas ${ }^{1}$ sobre o conjunto $\omega$-limite. $\mathrm{O}$ primeiro teorema nos dá uma maneira alternativa para definir o conjunto $\omega$-limite.

Teorema 2.2.3. (LASALLE, 1976) $\omega\left(x_{0}\right)=\cap_{0<\tau<\infty} \overline{\varphi\left([\tau, \infty), x_{0}\right)}$.

Demonstração. Suponha que $p \in \omega\left(x_{0}\right)$, então pela Definição 2.2.4, existe uma sequência $\left\{t_{j}\right\}$, com $t_{j} \longrightarrow+\infty$ quando $j \longrightarrow+\infty$ tal que $\varphi\left(t_{j}, x_{0}\right) \longrightarrow p$ quando $j \longrightarrow+\infty$. Logo, para qualquer $\tau \in[0,+\infty)$, existe um número inteiro $N>0$ tal que $t_{j} \in[\tau,+\infty)$ para todo $j \geqslant N$. Assim, $\varphi\left(t_{j}, x_{0}\right) \in \varphi\left([\tau,+\infty), x_{0}\right)$ para todo $j \geqslant N$. Portanto, $p \in$ $\overline{\varphi\left([\tau,+\infty), x_{0}\right)}$. Como $\tau$ pode ser escolhido arbitrariamente grande, tem-se que $p \in$ $\cap_{0<\tau<+\infty} \overline{\varphi\left([\tau,+\infty), x_{0}\right)}$. Suponha agora que $p \in \cap_{0<\tau<+\infty} \overline{\varphi\left([\tau,+\infty), x_{0}\right)}$. Então dado $\tau>0$ arbitrário, $p \in \overline{\varphi\left([\tau,+\infty), x_{0}\right)}$. Em particular, dado uma sequência $\left\{\tau_{j}\right\}$, com $\tau_{j} \longrightarrow+\infty$ quando $j \longrightarrow+\infty$, existe $t_{j} \in\left[\tau_{j},+\infty\right)$ tal que $\left\|\varphi\left(t_{j}, x_{0}\right)-p\right\| \leqslant \frac{1}{j}$. Obviamente, $t_{j} \longrightarrow+\infty$ quando $j \longrightarrow+\infty$ e, por construção, $\varphi\left(t_{j}, x_{0}\right) \longrightarrow p$ quando $j \longrightarrow+\infty$. Portanto, $p \in \omega\left(x_{0}\right)$.

O próximo teorema exibe propriedades para o conjunto $\omega$-limite de uma solução qualquer $\varphi\left(t, x_{0}\right)$ de (2.1). De maneira análoga, valem as mesmas propriedades para o conjunto $\alpha$-limite.

Teorema 2.2.4. (LASALLE, 1976) O conjunto $\omega$-limite de uma solução $\varphi\left(t, x_{0}\right)$ do sistema autônomo (2.1) é fechado e invariante.

Demonstração. Como a interseção de conjuntos fechados é um conjunto fechado, obtémse do teorema anterior que $\omega\left(x_{0}\right)$ é um conjunto fechado. Para provar que $\omega\left(x_{0}\right)$ é um conjunto invariante, seja $p \in \omega\left(x_{0}\right)$. Então de acordo com a Definição 2.2.4 existe uma sequência $\left\{t_{j}\right\}$, com $t_{j} \longrightarrow+\infty$ quando $j \longrightarrow+\infty$ tal que $\varphi\left(t_{j}, x_{0}\right) \longrightarrow p$ quando $j \longrightarrow$ $+\infty$. Seja $\varphi(t, p)$ a solução de (2.1) passando por $p$. Vamos mostrar que $\varphi(t, p) \in \omega\left(x_{0}\right)$ para todo $t \in \mathbb{R}$. Seja $\tau$ um número real arbitrário. Da continuidade das soluções com relação às condições iniciais, tem-se que dado $\varepsilon>0$, arbitrariamente pequeno, existe $\delta>0$ tal que $\|q-p\|<\delta$ implica em $\|\varphi(\tau, q)-\varphi(\tau, p)\|<\varepsilon$. Se escolhermos $\varepsilon_{m}=\frac{1}{m}$

\footnotetext{
${ }^{1}$ As demonstrações dos próximos três teoremas foram retiradas de Alberto (2006).
} 
onde $m=1,2, \ldots$, então para cada inteiro $m$, existe número real $\delta_{m}>0$ e um inteiro $M>0$ (dependendo de $m$ ) tal que $\left\|\varphi\left(t_{M}, x_{0}\right)-p\right\|<\delta_{m}$ e portanto $\left\|\varphi\left(\tau, \varphi\left(t_{M}, x_{0}\right)\right)-\varphi(\tau, p)\right\|<$ $\frac{1}{m}$. Pode-se, sem perda de generalidade, escolher a sequência $\left\{t_{M}\right\}$ estritamente crescente com $t_{M} \longrightarrow+\infty$ quando $m \longrightarrow+\infty$. Logo, tem-se que $\varphi\left(\tau, \varphi\left(t_{M}, x_{0}\right)\right)=\varphi\left(\tau+t_{M}, x_{0}\right) \longrightarrow$ $\varphi(\tau, p)$ quando $m \longrightarrow+\infty$. Portanto, $\varphi(t, p) \in \omega\left(x_{0}\right)$. A arbitrariedade da escolha de $x_{0}$ completa a demonstração mostrando que, para qualquer $p \in \omega\left(x_{0}\right), \varphi(t, p) \in \omega\left(x_{0}\right)$ para todo $\tau \in \mathbb{R}$.

Se a solução $\varphi\left(t, x_{0}\right)$ de (2.1) é limitada, podemos demonstrar que o conjunto $\omega$-limite possui algumas propriedades adicionais.

Teorema 2.2.5. (LASALLE, 1976) Se a solução $\varphi\left(t, x_{0}\right)$ de (2.1) é limitada, então o conjunto $\omega$-limite é não-vazio, conexo, fechado, limitado e invariante. Além disso, $d\left(\varphi\left(t, x_{0}\right), \omega\left(x_{0}\right)\right) \longrightarrow 0$ quando $t \longrightarrow+\infty$, onde d é a distância entre conjuntos definida na Seção 2.1.7.

Demonstração. Já provamos no teorema anterior que o conjunto $\omega\left(x_{0}\right)$ é fechado e invariante. O conjunto $\omega\left(x_{0}\right)$ é não vazio, pois toda sequência em um conjunto compacto possui subsequência convergente. A limitação da solução garante naturalmente a limitação do conjunto limite. Vamos provar que $d\left(\varphi\left(t, x_{0}\right), \omega\left(x_{0}\right)\right) \longrightarrow 0$ quando $t \longrightarrow+\infty$. Para isto, suponha, por contradição, que $\varphi\left(t, x_{0}\right)$ não se aproxima de $\omega\left(x_{0}\right)$ quando $t \longrightarrow+\infty$. Então, dada uma sequência de tempos $\left\{T_{n}\right\}$, com $T_{n} \longrightarrow+\infty$ quando $n \rightarrow+\infty$, existe $\varepsilon>0$ e $t_{n}>T_{n}$ tal que $d\left(\varphi\left(t_{n}, x_{0}\right), \omega\left(x_{0}\right)\right)>\varepsilon$ para todo $n=1,2,3, \ldots$ Como $\varphi\left(t_{n}, x_{0}\right)$ é uma sequência limitada em $\mathbb{R}^{n}$, então possui subsequência convergente, ou seja, existe $p \in \mathbb{R}^{n} \mathrm{e}$ subsequência $\left\{t_{n_{j}}\right\}$ de $\left\{t_{n}\right\}$ tal que $\varphi\left(t_{n_{j}}, x_{0}\right) \longrightarrow p$ quando $n_{j} \longrightarrow+\infty$. Mas por definição, $p \in \omega\left(x_{0}\right)$, portanto chegamos a uma contradição provando o que se queria. Resta-nos provar que o conjunto $\omega\left(x_{0}\right)$ é conexo. Suponha que $\omega\left(x_{0}\right)$ é desconexo, ou seja, $\omega\left(x_{0}\right)$ pode ser escrito como sendo a união de dois conjuntos, fechados e não vazios. Sejam, $A_{1}$ e $A_{2}$ estes conjuntos. Como eles são disjuntos e $\mathbb{R}^{n}$ é um espaço normal, existem abertos disjuntos $U_{1}$ e $U_{2}$ tal que $A_{1} \subset U_{1}$ e $A_{2} \subset U_{2}$. Como $d\left(\varphi\left(t, x_{0}\right), \omega\left(x_{0}\right)\right) \longrightarrow 0$ quando $t \longrightarrow+\infty$, então existe $T>0$ tal que $\varphi\left(t, x_{0}\right) \in U_{1} \cup U_{2}$ para todo $t>T$. Como a aplicação $t \longrightarrow \varphi\left(t, x_{0}\right)$ é contínua e $U_{1}$ e $U_{2}$ são disjuntos, conclui-se que $\varphi\left(t, x_{0}\right)$ pertence apenas a um dos abertos para todo $t>T$. Suponha sem perda de generalidade que $\varphi\left(t, x_{0}\right) \in U_{1}$ para todo $t>T$. Então, necessariamente $A_{2}$ é um conjunto vazio. Isto nos leva a uma contradição e portanto o conjunto $\omega$-limite é conexo.

A limitação da solução é um pré-requisito fundamental para demonstrar o resultado anterior. A Figura 2.2 mostra um exemplo de uma solução ilimitada que possui um conjunto $\omega$-limite desconexo. Mas precisamente, o conjunto $\omega$-limite é constituído pela união de duas retas disjuntas. O conjunto $\omega$-limite é ilimitado e ainda, dado $T>0$ arbitrariamente grande e $\varepsilon>0$, existe $t>T$ tal que $d\left(\varphi\left(t, x_{0}\right), \omega\left(x_{0}\right)\right)>\varepsilon$, ou seja, a solução não se aproxima do conjunto $\omega$-limite quando o tempo tende ao infinito. 


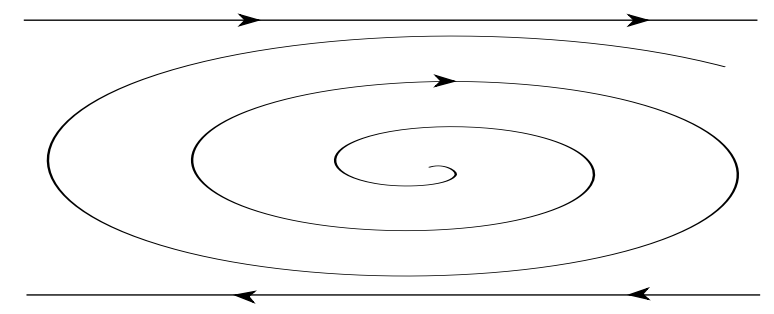

Figura 2.2: Conjunto $\omega$-limite desconexo e ilimitado.

\subsubsection{Equilíbrios e Estabilidade Local}

Na literatura existem diversas definições de estabilidade, nesse texto utilizaremos a definição de estabilidade no sentido de Lyapunov.

A grosso modo, uma solução $\varphi(t)$ do sistema (2.1) é estável quando toda solução com valores iniciais próximos aos de $\varphi(t)$ está definida para todo $t \geqslant 0$ e permanece próxima de $\varphi(t)$ para todo $t \geqslant 0$. Formalmente temos a seguinte definição:

Definição 2.2.6. (Estabilidade) Seja $\varphi(t)$ um solução de (2.1) definida para $t \geqslant 0$. Diz-se que $\varphi(t)$ é estável se para todo $\varepsilon>0$ existir $\delta>0$ tal que se $\psi(t)$ é solução de (2.1) e $\|\psi(0)-\varphi(0)\|<\delta$ então $\psi(t)$ está definida para todo $t \geqslant 0 e\|\psi(t)-\varphi(t)\|<\varepsilon$ para todo $t \geqslant 0$, ver Figura 2.3. Se além disso existir $\delta_{1}>0$ tal que $\|\psi(0)-\varphi(0)\|<\delta_{1}$ implica $\lim _{t \longrightarrow \infty}\|\psi(t)-\varphi(t)\|=0$, então $\varphi$ diz-se assintoticamente estável, ver Figura 2.4

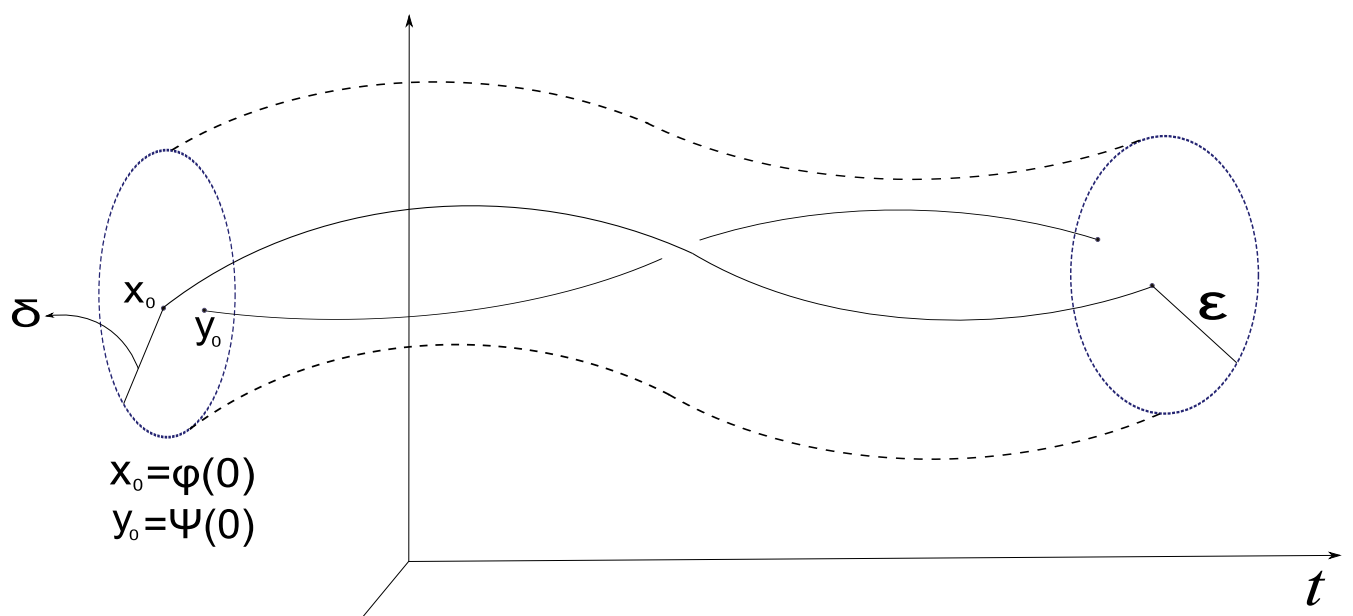

Figura 2.3: Solução estável.

Um caso especial de soluções que apresentaremos a seguir são os pontos de equilíbrio. É para este tipo de solução que estamos interessados em estudar estabilidade.

Definição 2.2.7. Um ponto $x^{*} \in \mathbb{R}^{n}$ é um ponto de equilíbrio do sistema (2.1) se $f\left(x^{*}\right)=0$. 


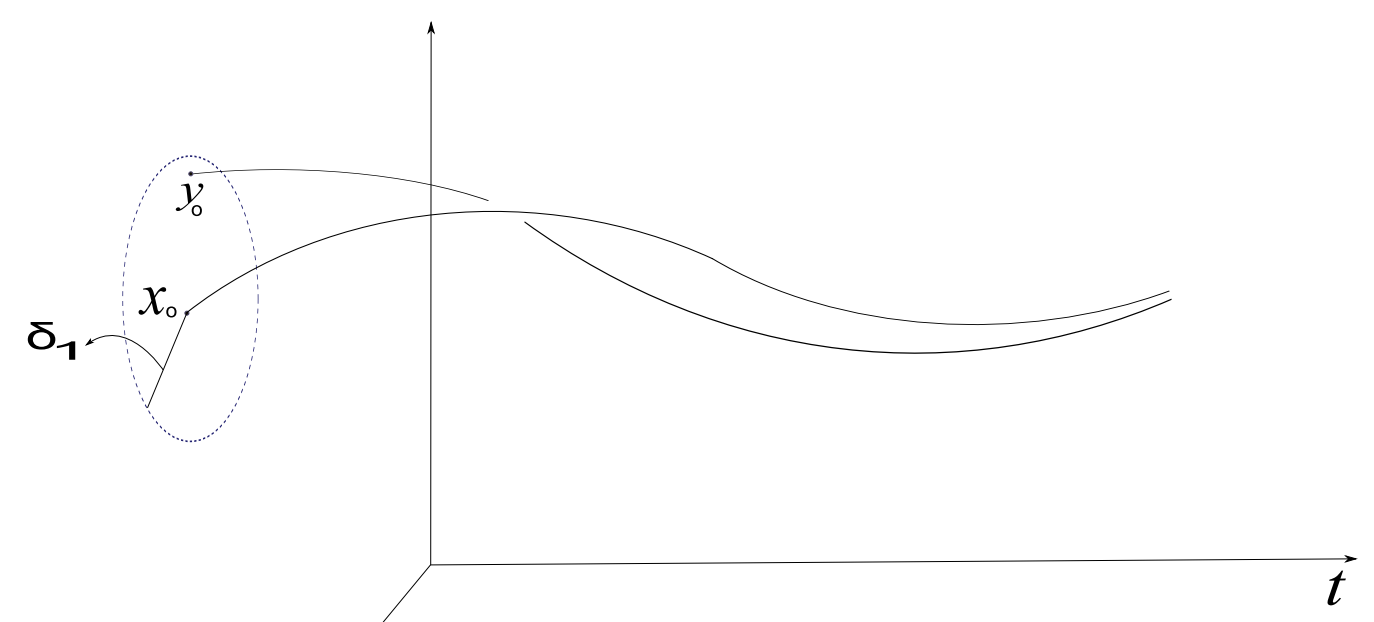

Figura 2.4: Solução assintoticamente estável.

Se $x^{*} \in \mathbb{R}^{n}$ é um ponto de equilíbrio do sistema (2.1), então a solução $\varphi$ de (2.1) iniciando em $x^{*}$ no tempo $t=0$ é a função constante, ou seja, $\varphi(t)=x^{*}$ para todo $t \in \mathbb{R}$. Sem maiores dificuldades, pode-se verificar que o ponto de equilíbrio é um conjunto invariante de (2.1).

Os conceitos propostos abaixo caracterizam a estabilidade de pontos de equilíbrio.

Definição 2.2.8. Um ponto de equilíbrio $x^{*}$ de (2.1) é estável se, para cada $\varepsilon>0$, existir um $\delta>0$ tal que, para toda condição inicial $x_{0}$ satisfazendo $\left\|x_{0}-x^{*}\right\|<\delta$ tem-se $\left\|\varphi\left(t, x_{0}\right)-x^{*}\right\|<\varepsilon$ para todo $t \geqslant 0$.

A Figura 2.5 ilustra a Definição 2.2.8. Soluções iniciando dentro da bola de raio $\delta$ não podem sair de dentro da bola de raio $\varepsilon$. Observe que as soluções neste caso não precisam se aproximar do ponto de equilíbrio $x^{*}$ quando o tempo $t \longrightarrow+\infty$.

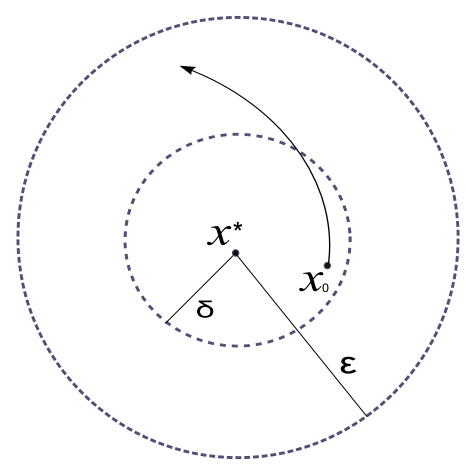

Figura 2.5: Ponto de equilíbrio estável.

A próxima definição é de ponto de equilíbrio instável.

Definição 2.2.9. Um ponto de equilíbrio $x^{*}$ do sistema (2.1) é instável se ele não é estável.

Uma outra propriedade interessante dos pontos de equilíbrio é a atratividade. 
Definição 2.2.10. Um ponto de equilíbrio $x^{*}$ do sistema (2.1) é atrativo se existir $\delta>0$ tal que

$$
x_{0} \in B\left(x^{*} ; \delta\right) \Longrightarrow \varphi\left(t, x_{0}\right) \longrightarrow x^{*} \text { quando } t \longrightarrow+\infty \text {. }
$$

A Figura 2.6 ilustra a Definição 2.2.10. Soluções iniciando dentro da bola de raio $\delta$ tendem para o ponto de equilíbrio $x^{*}$ quando o tempo $t \longrightarrow+\infty$. Observe que as soluções iniciando dentro da bola de raio $\delta$ podem se afastar do ponto de equilíbrio $x^{*}$ quando o tempo cresce, inclusive as soluções podem sair de dentro da bola de raio $\delta$, mas retornam e se aproximam do ponto de equilíbrio quando o tempo $t \longrightarrow+\infty$.

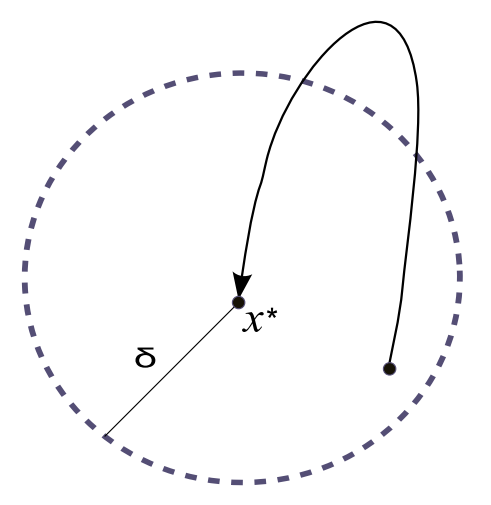

Figura 2.6: Ponto de equilíbrio atrativo.

Estabilidade não implica em atratividade e atratividade também não implica em estabilidade.

Combinando as Definições 2.2.8 e 2.2.10 temos o conceito de estabilidade assintótica.

Definição 2.2.11. Um ponto de equilíbrio de (2.1) é assintoticamente estável se for estável e atrativo.

A Figura 2.7 ilustra a Definição 2.2.11. Soluções iniciando dentro da bola de raio $\delta$ não saem de dentro da bola de raio $\varepsilon$ e tendem para o ponto de equilíbrio $x^{*}$ quando $o$ tempo $t \longrightarrow+\infty$. Em outras palavras, as soluções iniciando próximo a $x^{*}$, não podem se afastar do ponto de equilíbrio $x^{*}$ e obrigatoriamente se aproximam de $x^{*}$ quando o tempo $t \longrightarrow+\infty$.

Em algumas situações, deseja-se que não apenas o ponto de equilíbrio seja assintoticamente estável mas que todas as soluções tendam para este equilíbrio quando o tempo tende ao infinito. Para isto, define-se o conceito de estabilidade global assintótica.

Definição 2.2.12. Um ponto de equilíbrio de (2.1) é globalmente assintoticamente estável se ele é estável e para todo $x_{0} \in \mathbb{R}^{n}, \varphi\left(t, x_{0}\right) \longrightarrow x^{*}$ quando $t \longrightarrow+\infty$.

Da definição anterior, pode-se concluir que se $x^{*}$ é um ponto de equilíbrio globalmente assintoticamente estável de (2.1) então ele é único. 


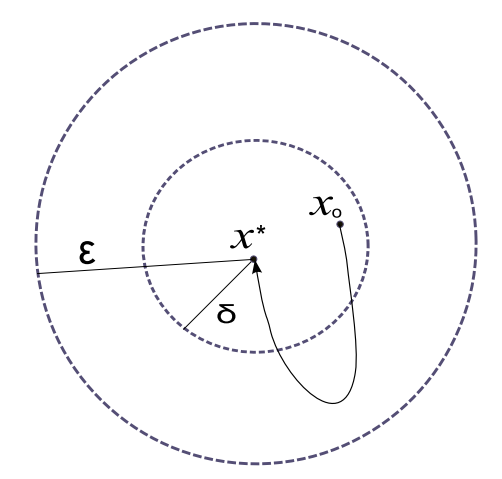

Figura 2.7: Ponto de equilíbrio assintoticamente estável.

Uma classe importante de pontos de equilíbrio são os pontos de equilíbrio hiperbólicos. Seja $x^{*}$ um ponto de equilíbrio do sistema (2.1) e considere o sistema linearizado em torno de $x^{*}$ :

$$
\dot{z}=J\left(x^{*}\right) z
$$

onde $z:=x-x^{*}$ e $J\left(x^{*}\right)$ é a matriz Jacobiana de $f(x)$ calculada no ponto de equilíbrio $x^{*}$.

Definição 2.2.13. Um ponto de equilíbrio $x^{*}$ de (2.1) é hiperbólico se todos os autovalores da matriz Jacobiana do sistema linearizado associado (2.2) possuem parte real não nula.

Os pontos de equilíbrio hiperbólicos tem uma propriedade importante que decorre do teorema de Hartman \& Grobman (PERKO, 1991), isto é, o comportamento do sistema (2.1), próximo a um ponto de equilíbrio hiperbólico $x^{*}$ é qualitativamente equivalente ao do sistema linearizado associado. Portanto, do ponto de vista local, a análise do comportamento dinâmico do sistema (2.1) resume-se, sob certas condições, à autoanálise do sistema linearizado associado.

Como consequência direta do Teorema da Função Inversa (LIMA, 2006), tem-se que pontos de equilíbrio hiperbólicos são necessariamente pontos de equilíbrio isolados, isto é, existe uma vizinhança do equilíbrio hiperbólico que não contém outro ponto de equilíbrio.

Definição 2.2.14. Um ponto de equilíbrio $x^{*}$ do sistema (2.1) é do tipo $k$ se a matriz Jacobiana $J\left(x^{*}\right)$ do sistema linearizado associado possui $k$ autovalores com parte real positiva e $n-k$ autovalores com parte real negativa.

Um resultado que pode ser encontrado em (PERKO, 1991) é que, se $x^{*}$ é um ponto de equilíbrio estável de (2.1), então nenhum autovalor da matiz Jacobiana $J\left(x^{*}\right)$ tem parte real positiva. Equivalentemente, se a Jacobiana de um ponto de equilíbrio $x^{*}$ de (2.1) tem pelo menos um autovalor com parte real positiva então $x^{*}$ é instável. Para o caso particular de pontos de equilíbrio hiperbólicos tem-se o seguinte resultado.

Teorema 2.2.6. (PERKO, 1991) Um ponto de equilíbrio hiperbólico $x^{*}$ de (2.1) é assintoticamente estável se, e somente se, é do tipo 0. 
Pelo Teorema 2.2.6 e observação anterior, podemos concluir que um ponto de equilíbrio hiperbólico $x^{*}$ de (2.1) ou é assintoticamente estável ou é instável.

\subsubsection{Variedades Invariantes}

Nesta seção, daremos continuidade a seção anterior no que diz respeito ao estudo do comportamento dinâmico nas vizinhanças de pontos de equilíbrio. O resultado principal que exploraremos é a existência de variedades invariantes nas vizinhanças de um ponto de equilíbrio que são fundamentais para o estudo da estabilidade e também para a caracterização da fronteira da região de estabilidade.

Dado $x^{*}$ um ponto de equilíbrio de (2.1) e $J\left(x^{*}\right)$ a Jacobiana do sistema linearizado associado (2.2), o espaço $\mathbb{R}^{n}$ pode ser decomposto como soma direta de três subespaços denotados por $E^{s}\left(x^{*}\right), E^{u}\left(x^{*}\right)$ e $E^{c}\left(x^{*}\right)$ que são invariantes com relação a (2.2):

$$
\begin{gathered}
E^{s}\left(x^{*}\right)=\left[e_{1}, \ldots, e_{s}\right], \\
E^{u}\left(x^{*}\right)=\left[e_{s+1}, \ldots, e_{s+u}\right], \quad s+u+c=n \\
E^{c}\left(x^{*}\right)=\left[e_{s+u+1}, \ldots, e_{s+u+c}\right],
\end{gathered}
$$

onde $\left\{e_{1}, \ldots, e_{s}\right\}$ são os autovetores generalizados de $J\left(x^{*}\right)$ correspondentes aos autovalores de $J\left(x^{*}\right)$ com parte real negativa, $\left\{e_{s+1}, \ldots, e_{s+u}\right\}$ são os autovetores generalizados de $J\left(x^{*}\right)$ correspondentes aos autovalores de $J\left(x^{*}\right)$ com parte real positiva e o conjunto $\left\{e_{s+u+1}, \ldots, e_{s+u+c}\right\}$ são os autovetores generalizados de $J\left(x^{*}\right)$ correspondentes aos autovalores de $J\left(x^{*}\right)$ com parte real nula. $E^{s}\left(x^{*}\right), E^{u}\left(x^{*}\right)$ e $E^{c}\left(x^{*}\right)$ são chamados de subespaço estável, instável e central, respectivamente.

Observação 2.2.2. O subespaço vetorial gerado pelo autovetores $\left\{e_{1}, \ldots, e_{s}\right\}$ é denotado por $\left[e_{1}, \ldots, e_{s}\right]$.

Todas as soluções de (2.2) que começam no subespaço estável $E^{s}\left(x^{*}\right)$ de (2.2) permanecem em $E^{s}\left(x^{*}\right)$ para todo $t$ e se aproximam da origem quando $t \rightarrow+\infty$, e todas as soluções de (2.2) que começam no subespaço instável $E^{u}\left(x^{*}\right)$ de (2.2) permanecem em $E^{u}\left(x^{*}\right)$ para todo $t$ e se aproximam da origem quando $t \rightarrow-\infty$. Para o sistema não linear (2.1), continuam existindo, nas vizinhanças do ponto de equilíbrio, conjuntos invariantes com relação a (2.1). Estes conjuntos invariantes não tem estrutura de espaços vetoriais, mas estrutura de variedades, como veremos nos próximos teoremas.

O teorema a seguir, conhecido por Teorema da Variedade Estável, é um resultado clássico na teoria de equações diferenciais ordinárias, que demonstra a existência de variedades invariantes nas vizinhanças de um ponto de equilíbrio hiperbólico.

Teorema 2.2.7. (PERKO, 1991) Seja $x^{*}$ um ponto de equilíbrio hiperbólico de (2.1) do tipo $k$. Então existe uma variedade local $W_{\text {loc }}^{u}\left(x^{*}\right), k$-dimensional de classe $C^{1}$, tangente 
ao subespaço instável $E^{u}\left(x^{*}\right)$ em $x^{*}$, tal que $\varphi_{t}\left(W_{l o c}^{u}\left(x^{*}\right)\right) \subset W_{l o c}^{u}\left(x^{*}\right)$ para todo $t \leqslant 0$ e para todo $x_{0} \in W_{l o c}^{u}\left(x^{*}\right)$,

$$
\lim _{t \longrightarrow-\infty} \varphi_{t}\left(x_{0}\right)=x^{*}
$$

e existe uma variedade local $W_{\text {loc }}^{s}\left(x^{*}\right),(n-k)$-dimensional de classe $C^{1}$, tangente ao subespaço estável $E^{s}\left(x^{*}\right)$ em $x^{*}$, tal que $\varphi_{t}\left(W_{l o c}^{s}\left(x^{*}\right)\right) \subset W_{l o c}^{s}\left(x^{*}\right)$ para todo $t \geqslant 0$ e para todo $x_{0} \in W_{l o c}^{s}\left(x^{*}\right)$,

$$
\lim _{t \longrightarrow+\infty} \varphi_{t}\left(x_{0}\right)=x^{*}
$$

O Teorema 2.2.7 demonstra a existência das variedades $W_{l o c}^{s}\left(x^{*}\right)$ e $W_{l o c}^{u}\left(x^{*}\right)$ apenas em uma vizinhança do equilíbrio $x^{*}$, por isso são chamadas variedades locais estável e instável, respectivamente. Definimos a seguir variedade global estável e instável de $x^{*}$, ou simplesmente, a variedade estável e instável de $x^{*}$.

Definição 2.2.15. As variedades estável e instável de um ponto de equilíbrio hiperbólico $x^{*}$ do sistema (2.1) são definidas por:

$$
W^{s}\left(x^{*}\right)=\bigcup_{t \leqslant 0} \varphi_{t}\left(W_{l o c}^{s}\left(x^{*}\right)\right) \text { e } W^{u}\left(x^{*}\right)=\bigcup_{t \geqslant 0} \varphi_{t}\left(W_{l o c}^{u}\left(x^{*}\right)\right)
$$

respectivamente.

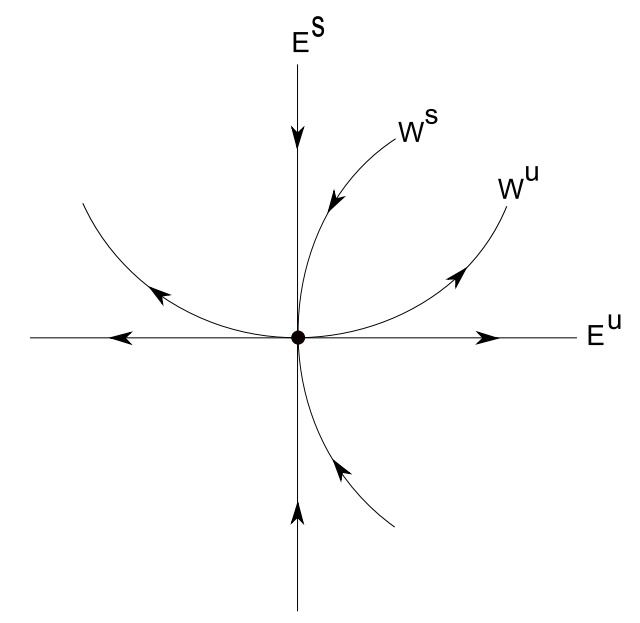

Figura 2.8: Variedade estável $W^{s}$ e variedade instável $W^{u}$.

A variedade estável $W^{s}\left(x^{*}\right)$ e a variedade instável $W^{u}\left(x^{*}\right)$ do ponto de equilíbrio hiperbólico $x^{*}$ são únicas e invariantes com relação a (2.1). Além disso, também podem ser escritas como

$$
W^{s}\left(x^{*}\right)=\left\{x_{0} \in \mathbb{R}^{n}: \varphi\left(t, x_{0}\right) \longrightarrow x^{*} \text { quando } t \longrightarrow+\infty\right\}
$$

e

$$
W^{u}\left(x^{*}\right)=\left\{x_{0} \in \mathbb{R}^{n}: \varphi\left(t, x_{0}\right) \longrightarrow x^{*} \text { quando } t \longrightarrow-\infty\right\},
$$


respectivamente, ver Figura 2.8.

No Teorema 2.2.7, o ponto de equilíbrio $x^{*}$ foi considerado hiperbólico. Para o caso de $x^{*}$ não hiperbólico, exibiremos a seguir um teorema que também garante a existência de variedades locais invariantes. Para detalhes e demonstração deste teorema ver (HIRSCH; PUGH; SHUB, 1970).

Teorema 2.2.8. (HIRSCH; PUGH; SHUB, 1970) Seja $x^{*}$ um ponto de equilíbrio não hiperbólico de (2.1). Então existem variedades locais invariantes $W_{l o c}^{s}\left(x^{*}\right), W_{l o c}^{c s}\left(x^{*}\right)$, $W_{\text {loc }}^{c}\left(x^{*}\right), W_{\text {loc }}^{u}\left(x^{*}\right)$ e $W_{\text {loc }}^{c u}\left(x^{*}\right)$ de classe $C^{1}$, tangentes a $E^{s}\left(x^{*}\right), E^{c}\left(x^{*}\right) \oplus E^{s}\left(x^{*}\right), E^{c}\left(x^{*}\right)$, $E^{u}\left(x^{*}\right)$ e $E^{c}\left(x^{*}\right) \oplus E^{u}\left(x^{*}\right)$ em $x^{*}$, respectivamente.

As variedades $W_{l o c}^{s}\left(x^{*}\right), W_{l o c}^{c s}\left(x^{*}\right), W_{l o c}^{c}\left(x^{*}\right), W_{l o c}^{u}\left(x^{*}\right)$ e $W_{l o c}^{c u}\left(x^{*}\right)$ são chamadas variedades locais estável, centro estável, central, instável e centro instável, respectivamente. Soluções iniciando em $W_{l o c}^{s}\left(x^{*}\right)$ tendem para $x^{*}$ quando $t \rightarrow+\infty$ e soluções iniciando em em $W_{l o c}^{u}\left(x^{*}\right)$ tendem para $x^{*}$ quando $t \rightarrow-\infty$. As variedades local estável e instável são únicas, mas a variedade local centro estável, central e centro instável não precisam ser.

Um exemplo que mostra a não unicidade da variedade local central, é o seguinte sistema

$$
\begin{aligned}
& \dot{x}=x^{2} \\
& \dot{y}=-y
\end{aligned}
$$

onde $x$ e $y$ são números reais.

Para $x<0$, todas as trajetórias do sistema (2.5) se aproximam da origem. Para $x>0$, a única trajetória que se aproxima da origem é o eixo- $x$, ver Figura 2.9. Podemos obter a variedade central juntando qualquer trajetória no lado esquerdo do plano com a metade positiva do eixo- $x$, portanto, a variedade central não é única.

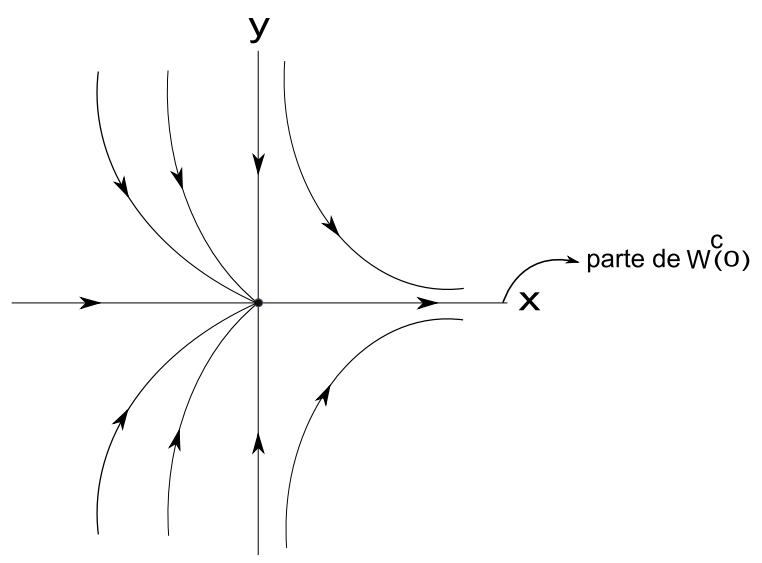

Figura 2.9: Retrato de fase do sistema (2.5). 


\subsection{Transversalidade}

Nesta seção, apresentaremos o conceito de transversalidade, que será usado com frequência ao longo do texto para a caracterização da fronteira da região de estabilidade. Para complemento ou maiores detalhes das definições e resultados expostos nesta seção ver (WIGGINS, 2003) e (GUILLEMIN; POLLACK, 1974).

Definição 2.3.1. Seja $p \in \mathbb{R}^{n}$. Duas variedades $M$ e $N$ em $\mathbb{R}^{n}$ de classe $C^{r}(r \geqslant 1)$ são transversais em $p$ se $p \notin M \cap N$; ou se $p \in M \cap N$, então $T_{p}(M)+T_{p}(N)=\mathbb{R}^{n}$, onde $T_{p}(M)$ e $T_{p}(N)$ denotam o espaço tangente de $M$ e $N$, respectivamente, no ponto $p . M$ e $N$ satisfazem a condição de transversalidade se elas são transversais em cada ponto $p \in \mathbb{R}^{n}$.

A Figura 2.10 mostra duas curvas $M$ e $N$ em $\mathbb{R}^{2}$. A figura à esquerda mostra as curvas $M$ e $N$ tendo interseção transversal em um ponto $p \in M \cap N$, e a figura à direita mostra as curvas $M$ e $N$ tendo interseção não transversal em um ponto $p \in M \cap N$.

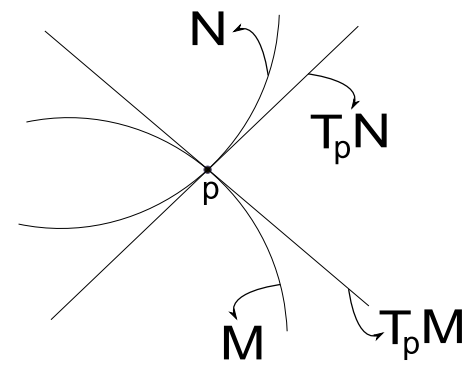

transversal emp

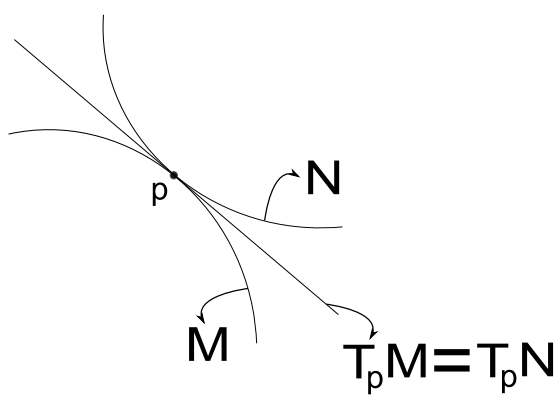

não transversal em $p$

Figura 2.10: Curvas $M$ e $N$ em $\mathbb{R}^{2}$.

Um resultado que envolve dimensão e interseção transversal de variedades será exibido no próximo lema.

Lema 2.3.1. (WIGGINS, 2003) Se duas variedades $M$ e $N$ em $\mathbb{R}^{n}$ de classe $C^{r}(r \geqslant 1)$ tem interseção não vazia, ou seja, $M \cap N \neq \emptyset$, e satisfazem a condição de transversalidade então

$$
\operatorname{dim}(M \cap N)=\operatorname{dim} M+\operatorname{dim} N-n .
$$

A mais importante característica da transversalidade é que esta persiste sob pequenas pertubações (WIGGINS, 2003). Esta propriedade de persitência da transversalidade sob pequenas pertubações será bastante explorada ao longo deste trabalho. Exibiremos a seguir um exemplo para ilustrar esta característica da condição de transversalidade.

Exemplo 2.3.1. Sejam $M$ a reta de equação $y=1$ em $\mathbb{R}^{2}$ e $N$ o gráfico da função $f(x)=$ $x^{2}$, ver Figura 2.11. Então $M$ e $N$ se interceptam transversalmente nos pontos $q=(-1,1)$ e $p=(1,1)$. Perturbando a reta $M$ e a função $f$ com relação a um parâmetro escalar 
$\lambda$, ou seja, $M_{\lambda}$ é reta perturbada de equação $y=1+\lambda$ e $N_{\lambda}$ é o gráfico da função perturbada $f(x)=x^{2}+\lambda$, temos ainda que $M_{\lambda}$ e $N_{\lambda}$ se interceptam transversalmente nos pontos $q=(-1,1+\lambda)$ e $p=(1,1+\lambda)$.

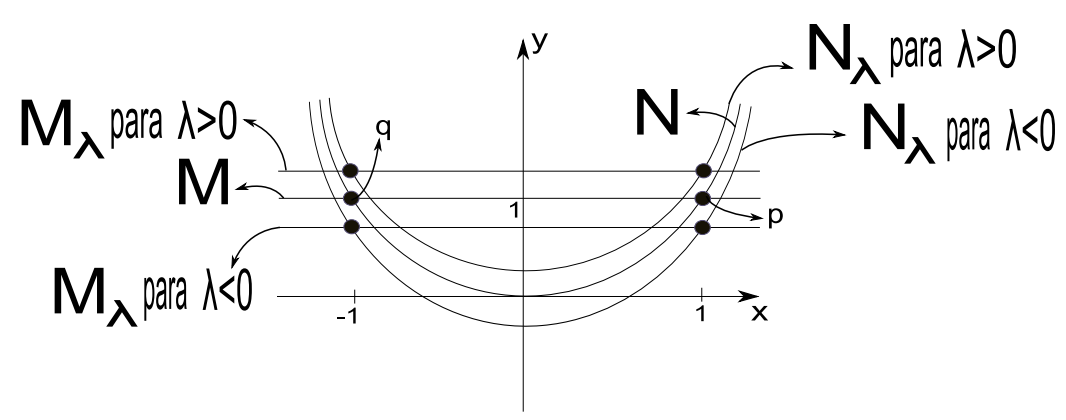

Figura 2.11: Ilustração do Exemplo 2.3.1.

A seguir um exemplo onde a condição de transversalidade é violada é apresentada.

Exemplo 2.3.2. Sejam $M$ o eixo- $x$ em $\mathbb{R}^{2}$ e $N$ o gráfico da função $f(x)=x^{3}$, ver Figura 2.12. Então M e $N$ se interceptam na origem em $\mathbb{R}^{2}$, mas elas não se interceptam transversalmente na origem, pois o espaço tangente de $M$ é apenas o eixo-x e o espaço tangente de $N$ é gerado pelo vetor $(1,0)$, $\operatorname{logo} T_{(0,0)} N=T_{(0,0)} M$ e, portanto, $T_{(0,0)} N+T_{(0,0)} M \neq \mathbb{R}^{2}$.

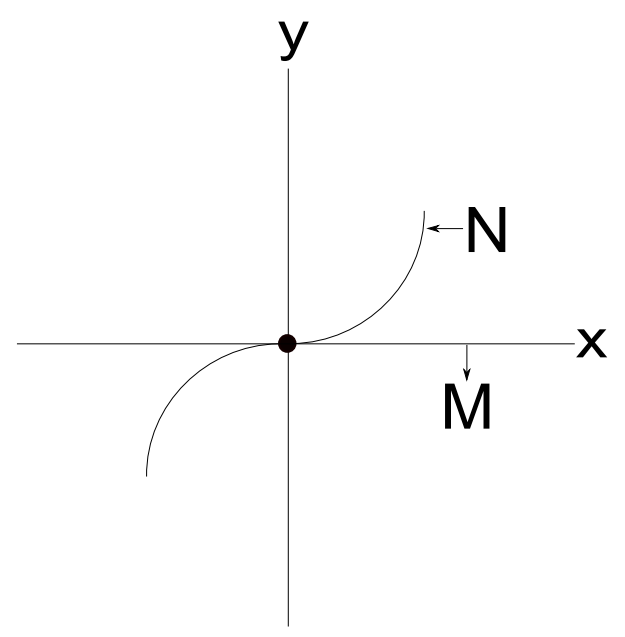

Figura 2.12: Variedades não transversais.

\subsection{Propriedade genérica}

Nesta seção discutiremos o conceito de propriedade genérica que aparecerá ao longo do trabalho. Maiores detalhes a respeito dos tópicos aqui desenvolvidos são encontrados em (WIGGINS, 2003; PALIS; MELO, 1977; PEIXOTO, 1967). Começaremos a seção com a definição de conjunto residual. 
Definição 2.4.1. Sejam $X$ um espaço topológico e $U$ um subconjunto de $X$. $U$ é chamado um conjunto residual se este contém uma interseção enumerável de conjuntos abertos $e$ densos em $X$.

A seguir, apresenta-se a definição de propriedade genérica.

Definição 2.4.2. Seja X um espaço topológico. Dizemos que uma propriedade é genérica em $X$ se o conjunto dos pontos de X que satisfazem esta propriedade contém um subconjunto residual.

Grosseiramente falando, uma propriedade é genérica em um espaço se esta propriedade é satisfeita para quase todos os pontos do espaço.

Exemplos interessantes de propriedade genérica podem ser encontrados com detalhes em (PEIXOTO, 1967). Dentre os campos vetoriais de classe $C^{r}(r \geqslant 1)$, as seguintes propriedades são genéricas:

(1) Todos pontos de equilíbrio são hiperbólicos.

(2) A interseção das variedades estáveis e instáveis dos pontos de equilíbrio hiperbólicos satisfazem a condição de transversalidade. 



\section{REGIÃO DE ESTABILIDADE}

Neste capítulo, apresentaremos o conceito de região de estabilidade e algumas propriedades topológicas deste conjunto. O conjunto denominado de região de estabilidade também recebe o nome na literatura de domínio de atração, área de atração, região de atração e bacia de atração. A região de estabilidade é um subconjunto do espaço de estados cujas trajetórias iniciando dentro desse conjunto tendem para o conjunto atrativo quando o tempo tende para o infinito. Este capítulo discute a caracterização da região de estabilidade de sistemas dinâmicos autônomos do ponto de vista topológico. Na Seção 3.1 introduziremos o conceito de conjunto atrativo e atrator, e faremos algumas discussões desses conceitos. Na Seção 3.2 a definição de região de estabilidade será exibida assim como uma caracterização topológica da região de estabilidade e da fronteira da região de estabilidade. Uma caracterização dinâmica da fronteira da região de estabilidade será apresentada no próximo capítulo.

\subsection{Conjuntos Atrativos}

Antes de definir região de estabilidade apresentaremos, nesta seção, o conceito e algumas discussões sobre conjuntos atrativos e atratores. A definição de conjunto atrativo é o ponto de partida para se definir região de estabilidade.

Dado um ponto de equilíbrio atrativo $x^{*}$ de (2.1), que é um conjunto invariante e fechado, podemos afirmar pela Definição 2.2.10 que existe um bola aberta $B\left(x^{*} ; r\right)$ tal que toda trajetória começando dentro dessa bola tende para $x^{*}$ quando o tempo tende para o infinito. Generalizando a definição de ponto de equilíbrio atrativo, tem-se a definição de conjunto atrativo dada a seguir.

Definição 3.1.1. (ALBERTO, 2006) Um conjunto $H$, fechado, invariante com relação a (2.1) é um conjunto atrativo se existir uma vizinhança $U$ de $H$ tal que, para toda condição inicial $x_{0} \in U, \varphi\left(t, x_{0}\right) \longrightarrow H$ quando $t \longrightarrow+\infty$.

Na Definição 3.1.1, a expressão $\varphi\left(t, x_{0}\right) \longrightarrow H$ quando $t \longrightarrow+\infty$ significa que $d\left(\varphi\left(t, x_{0}\right), H\right) \longrightarrow 0$ quando $t \longrightarrow+\infty$, onde $d$ é a distância entre um ponto e um con- 
junto definida na Seção 2.1.7. A vizinhança $U$ da definição acima será chamada vizinhança atrativa de $H$.

Todo ponto de equilíbrio atrativo é um conjunto atrativo. O primeiro resultado dessa seção mostra que a união de conjuntos atrativos é ainda um conjunto atrativo.

Teorema 3.1.1. Sejam $H_{1}, H_{2} \subset \mathbb{R}^{n}$ conjuntos atrativos. Então $H=H_{1} \cup H_{2}$ é um conjunto atrativo.

Demonstração. Como $H_{1}$ e $H_{2}$ são conjuntos atrativos, em particular são fechados e invariantes. Logo é fácil ver que $H=H_{1} \cup H_{2}$ é também fechado e invariante, já que união finita de conjuntos fechados e invariantes é um conjunto fechado e invariante. A atratividade de $H_{1}$ garante a existência de vizinhança $U_{1}$ de $H_{1}$ tal que $x_{0} \in U_{1}$ implica $d\left(\varphi\left(t, x_{0}\right), H_{1}\right) \longrightarrow 0$ quando $t \longrightarrow+\infty$, ou seja, dado $\varepsilon>0$, existe $t_{1} \in \mathbb{R}$ tal que $d\left(\varphi\left(t, x_{0}\right), H_{1}\right)<\varepsilon$ para todo $t>t_{1}$. Como $H_{2}$ também é atrativo, também existe vizinhança $U_{2}$ de $H_{2}$ tal que $x_{0} \in U_{2}$ implica $d\left(\varphi\left(t, x_{0}\right), H_{2}\right) \longrightarrow 0$ quando $t \longrightarrow+\infty$, ou seja, para todo $\varepsilon>0$, existe $t_{2} \in \mathbb{R}$ tal que $d\left(\varphi\left(t, x_{0}\right), H_{2}\right)<\varepsilon$ para todo $t>t_{2}$. Considere $U=U_{1} \cup U_{2}$ uma vizinhança de $H=H_{1} \cup H_{2}$. Mostraremos agora que $U=U_{1} \cup U_{2}$ é uma vizinhança atrativa de $H=H_{1} \cup H_{2}$. Para isso, seja $x_{0} \in U$. Sem perda de generalidade podemos supor que $x_{0} \in U_{1}$. Como $d\left(\varphi\left(t, x_{0}\right), H_{1} \cup H_{2}\right) \leqslant d\left(\varphi\left(t, x_{0}\right), H_{1}\right)$, então para todo $\varepsilon>0$, existe $t_{1} \in \mathbb{R}$ tal que $d\left(\varphi\left(t, x_{0}\right), H_{1} \cup H_{2}\right) \leqslant d\left(\varphi\left(t, x_{0}\right), H_{1}\right)<\varepsilon$ para todo $t>t_{1}$, ou seja, $d\left(\varphi\left(t, x_{0}\right), H_{1} \cup H_{2}\right) \longrightarrow 0$ quando $t \longrightarrow+\infty$. Portanto, como $x_{0} \in U$ foi tomado genérico podemos afirmar que $U=U_{1} \cup U_{2}$ é uma vizinhança atrativa de $H=H_{1} \cup H_{2}$, o que finaliza a demonstração do teorema.

Como consequência direta do teorema anterior temos o seguinte resultado.

Corolário 3.1.1. Sejam $H_{1}, H_{2}, \ldots, H_{n} \subset \mathbb{R}^{n}$ conjuntos atrativos. Então o conjunto $H=$ $H_{1} \cup H_{2} \cup \ldots \cup H_{n}$ é atrativo.

Provamos que união finita de conjuntos atrativos é um conjunto atrativo. Num certo sentido, vamos estudar a implicação contrária, ou seja, dados dois conjuntos $H_{1}$ e $H_{2}$ fechados, invariantes e contidos em $\mathbb{R}^{n}$ tais que $H=H_{1} \cup H_{2}$ seja atrativo, será que $H_{1}$ e $\mathrm{H}_{2}$ são atrativos? Para responder essa pergunta, apresentamos o seguinte contra exemplo que pode ser encontrado em (GUCKENHEIMER; HOLMES, 1983).

Exemplo 3.1.1. Considere o sistema de equações diferenciais

$$
\begin{aligned}
& \dot{x}=y+x\left(1-\left(x^{2}+y^{2}\right)\right) \\
& \dot{y}=-x+y\left(1-\left(x^{2}+y^{2}\right)\right)
\end{aligned}
$$

onde $(x, y) \in \mathbb{R}^{2}$. 
Neste exemplo, o sistema (3.1) apresenta um ciclo limite estável que denotaremos por $H_{1}$ e um ponto de equilíbrio instável que é a origem e que denotaremos pelo conjunto $H_{2}=\{(0 ; 0)\}$. O conjunto $H=H_{1} \cup H_{2}$ é um conjunto atrativo, pois é um conjunto fechado e invariante e existe vizinhança atrativa $U=U_{1} \cup U_{2}$ de $H$, onde $U_{1}$ é um anel que contém o ciclo limite $H_{1}$ e $U_{2}$ uma vizinhança do ponto de equilíbrio $(0 ; 0)$, conforme mostra a Figura 3.1. Por outro lado, o conjunto $H_{2}$ não é um conjunto atrativo. Portanto, a resposta para a pergunta acima é negativa.

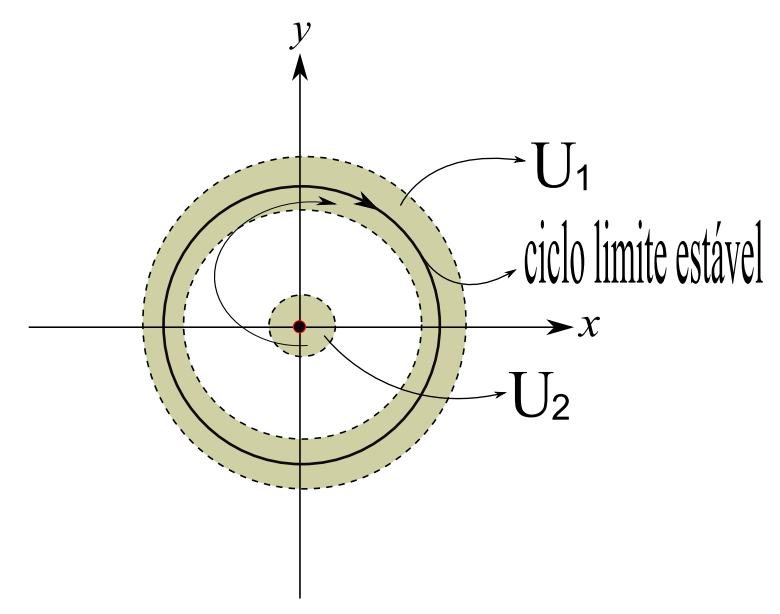

Figura 3.1: Vizinhança atrativa $U=U_{1} \cup U_{2}$ do conjunto atrativo $H=H_{1} \cup H_{2}$ do sistema (3.1) onde $H_{1}$ é um ciclo limite estável e $H_{2}=\{(0 ; 0)\}$ um ponto de equilíbrio instável.

Considere um outro exemplo de conjunto atrativo que pode ser encontrado em (WIGGINS, 2003).

Exemplo 3.1.2. Considere o seguinte sistema de equações diferenciais

$$
\begin{aligned}
& \dot{x}=x-x^{3} \\
& \dot{y}=-y
\end{aligned}
$$

onde $(x, y) \in \mathbb{R}^{2}$.

O sistema (3.2) tem um ponto de equilíbrio instável em $(0 ; 0)$ e dois pontos de equilíbrio atrativos em $(-1 ; 0)$ e $(1 ; 0)$. O conjunto $H=[-1,1] \times\{0\}$ é um conjunto atrativo, pois é fechado, invariante e cada trajetória que começa na vizinhança atrativa $U$, ver Figura 3.2, se aproxima de um dos pontos de equilíbrios $(-1 ; 0),(1 ; 0)$ ou $(0 ; 0)$. Em particular se aproximam de $H=[-1,1] \times\{0\}$. Com isso, existem subconjuntos $S_{1}=(-1,0) \times\{0\}$ e $S_{2}=(0,1) \times\{0\}$ que estão contidos no conjunto atrativo $H$, porém nenhuma trajetória começando na vizinhança atrativa se aproxima de pontos de $S_{1}$ e $S_{2}$ quando o tempo tende ao infinito.

O intuito de introduzir os dois exemplos anteriores é para mostrar que a Definição 3.1.1 apresenta algumas peculiaridades: $(i)$ Um conjunto atrativo desconexo pode possuir 


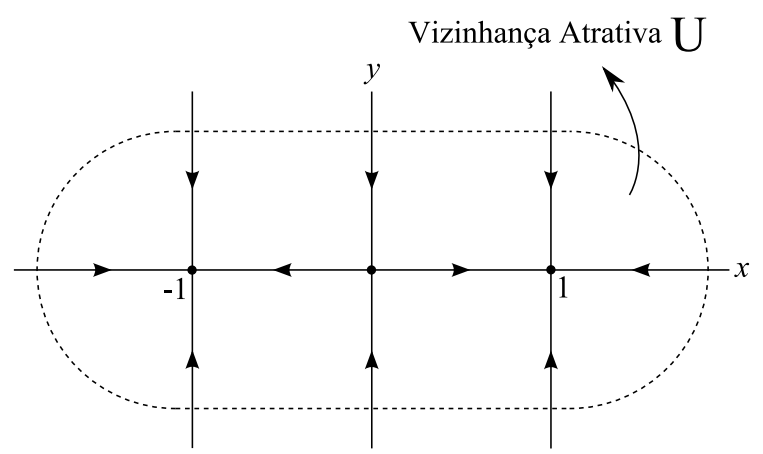

Figura 3.2: Vizinhança atrativa $U$ do conjunto atrativo $H=[-1,1] \times\{0\}$ do sistema (3.2).

uma de suas componentes conexas não atrativa (Exemplo 3.1.1), ou, (ii) podem existir subconjuntos de conjuntos atrativos, tal que as trajetórias começando na vizinhança atrativa não se aproximam de nenhum ponto desses subconjuntos quando o tempo tende para o infinito (Exemplo 3.1.2). Sendo assim, em certas ocasiões, dependendo do interesse e propósito de estudo, a Definição 3.1.1 pode não ser conveniente. Diante dessa discussão, apresentaremos o conceito de conjunto atrator que é mais forte que conjunto atrativo.

Definição 3.1.2. (WIGGINS, 2003) Um conjunto $H \subset \mathbb{R}^{n}$ é um atrator se $H$ é um conjunto atrativo e além disso

(i) $\varphi(t, U) \subset U$ para todo $t \geqslant 0$ e alguma vizinhança atrativa $U$ de $H$;

(ii) para quaisquer dois conjuntos abertos $V$ e $S$ em $H$

$$
\exists t \in \mathbb{R} \text { tal que } \varphi(t, V) \cap S \neq \emptyset \text {. }
$$

Os conjuntos atrativos apresentados no Exemplo 3.1.1 e Exemplo 3.1.2 não são atratores. A condição (ii) impõe uma condição especial de conexidade via órbitas do próprio sistema dinâmico.

O lema a seguir demonstra que um atrator não pode ser desconexo, em particular união de atratores disjuntos não pode ser um atrator.

Lema 3.1.1. Se $H \subset \mathbb{R}^{n}$ é um atrator então $H$ é conexo.

Demonstração. A prova será feita pela contrapositiva. Suponha que $H$ seja desconexo, então existe uma cisão não trivial de $H$, isto é, existem conjuntos abertos $A$ e $B$ em $H$, disjuntos e não vazios tais que $H=A \cup B$. Considere $x_{0} \in A$, como $H$ é invariante, $A$ e $B$ são disjuntos e aplicação $t \longrightarrow \varphi\left(t, x_{0}\right)$ é contínua podemos afirmar que $\varphi\left(t, x_{0}\right) \in A$ para todo $t \in \mathbb{R}$. Pela arbitrariedade de $x_{0}$ podemos afirmar que $\varphi(t, A) \subset A$ para todo $t \in \mathbb{R}$, isto é, $\varphi(t, A) \cap B=\emptyset \forall t \in \mathbb{R}$, o que contradiz o item (ii) da definição de atrator. Portanto, se $H$ é atrator então $H$ é conexo como queríamos demonstrar.

Corolário 3.1.2. Sejam $H_{1}$ e $H_{2}$ atratores disjuntos. Então $H=H_{1} \cup H_{2}$ não é um atrator. 
Antes de finalizar essa seção, queremos explorar ainda o seguinte exemplo que é devido a Vinograd (1957).

Exemplo 3.1.3. Considere o seguinte sistema de equações diferenciais

$$
\begin{aligned}
& \dot{x}=\frac{x^{2}(y-x)+y^{5}}{\left(x^{2}+y^{2}\right)\left[1+\left(x^{2}+y^{2}\right)^{2}\right]} \\
& \dot{y}=\frac{y^{2}(y-2 x)}{\left(x^{2}+y^{2}\right)\left[1+\left(x^{2}+y^{2}\right)^{2}\right]}
\end{aligned}
$$

onde $(x, y) \in \mathbb{R}^{2}$.

No sistema (3.3) a origem é um ponto de equilíbrio atrativo, já que para qualquer bola aberta de raio $r$ as trajetórias começando dentro da bola se aproximam da origem quando o tempo tende para o infinito, mas o conjunto $H=\{(0 ; 0)\}$ não é um conjunto atrator, visto que para as mesmas bolas abertas de raio $r$, existem trajetórias que começando dentro da bola saem dela para um certo tempo $T>0$, conforme Figura 3.3. Logo, nenhuma vizinhança $U$ de $H$ satisfaz o item (i) da Definição 3.1.2.

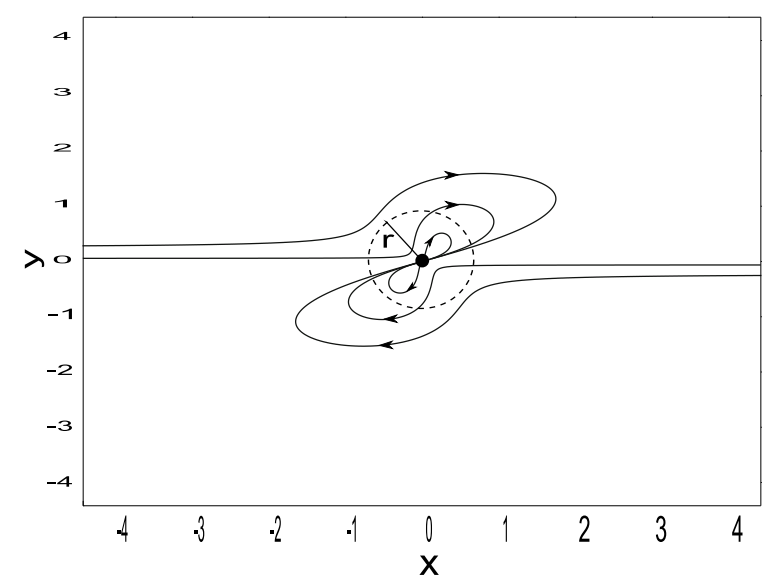

Figura 3.3: Ponto de equilíbrio atrativo do sistema (3.3).

Embora o conceito de atrator seja mais forte que o conceito de conjunto atrativo, o conceito de conjunto atrativo é suficiente para definir o conceito de região de estabilidade. Apesar das peculiaridades negativas do conceito de conjunto atrativo, definiremos a região de estabilidade de conjuntos atrativos. Como todo atrator é um conjunto atrativo, então a definição de região de estabilidade se aplica ao conceito de atrator.

\subsection{Caracterização topológica da região de estabilidade}

Nesta seção, exploraremos algumas propriedades topológicas da região de estabilidade e da sua fronteira. Vale registrar que algumas das propriedades topológicas da região de estabilidade que serão exploradas são de extrema relevância para os capítulos posteriores, como por exemplo, a propriedade da região de estabilidade ser um conjunto aberto. Os resultados explorados aqui, revisam algumas propriedades topológicas da região de 
estabilidade para pontos de equilíbrio atrativos que já existem na literatura, ver (CHIANG; HIRSCH; WU, 1988) e (ZABORSZKY et al., 1988), generalizam algumas dessas propriedades para conjuntos atrativos e por fim caracterizam a região de estabilidade para conjuntos atrativos que se decompõem como união finita de conjuntos atrativos.

Ao contrário dos sistemas dinâmicos lineares, um ponto de equilíbrio assintoticamente estável de um sistema não linear raramente é globalmente assintoticamente estável. Conhecer o conjunto dos pontos no espaço de estados cujas trajetórias, que começam nesses pontos, se aproximam de um ponto de equilíbrio assintoticamente estável quando o tempo tende ao infinito possibilita analisar de uma maneira muito mais completa e global o comportamento dinâmico do sistema em estudo. Nesse sentido, temos a seguinte definição.

Definição 3.2.1. Seja $H$ um conjunto atrativo de (2.1). A área de atração de $H$ é o conjunto $A(H)$ definido como

$$
A(H)=\left\{x_{0} \in \mathbb{R}^{n}: \varphi\left(t, x_{0}\right) \longrightarrow H \text { quando } t \longrightarrow+\infty\right\}
$$

Observe que $A(H)$ é um conjunto não vazio e contém pontos que não estão em $H$, já que toda vizinhança atrativa $U$ de $H$ é tal que $U \subset A(H)$.

Apresentaremos a seguir alguns teoremas sobre a invariância da região de estabilidade.

Teorema 3.2.1. (CHIANG; HIRSCH; WU, 1988) Seja H um conjunto atrativo de (2.1). Então $A(H)$ é um conjunto invariante.

Demonstração $^{1}$. Seja.$x_{0} \in A(H)$, ou seja, $d\left(\varphi\left(t, x_{0}\right), H\right) \longrightarrow 0$ quando $t \longrightarrow+\infty$. Seja $p=\varphi\left(s, x_{0}\right)$ para algum $s \in \mathbb{R}$ arbitrário. Então, da propriedade da solução $\varphi$, tem-se que $\varphi(t, p)=\varphi\left(t, \varphi\left(s, x_{0}\right)\right)=\varphi\left(t+s, x_{0}\right)$. Logo, para $s$ fixo,

$$
d(\varphi(t, p), H)=d\left(\varphi\left(t+s, x_{0}\right), H\right) \longrightarrow 0 \text { quando } t \longrightarrow+\infty .
$$

Portanto, $p \in A(H)$ e da arbitrariedade da escolha de $x_{0}$ podemos afirmar que $A(H)$ é invariante com relação ao sistema (2.1).

O resultado a seguir permite tirar conclusões sobre a invariância do fecho da região de estabilidade.

Teorema 3.2.2. Seja $H$ um conjunto invariante com relação ao sistema (2.1). Então $\bar{H}$ também é um conjunto invariante.

Demonstração. Seja $x_{0} \in \bar{H}$ e $T \in \mathbb{R}$ arbitrário. Mostraremos que $\varphi\left(T, x_{0}\right) \in \bar{H}$, isto é, para todo $\varepsilon>0$ suficientemente pequeno, existe $z^{*} \in H$ tal que $\left\|z^{*}-\varphi\left(T, x_{0}\right)\right\|<\varepsilon$. De fato, da continuidade das soluções com relação às condições iniciais, dado $\varepsilon>0$ existe

\footnotetext{
${ }^{1}$ A demonstração foi retirada do trabalho de Alberto (2006)
} 
$r>0$ tal que $\left\|x-x_{0}\right\|<r$ implica em $\left\|\varphi(t, x)-\varphi\left(t, x_{0}\right)\right\|<\varepsilon$ para todo $t \in[-T, T]$. Por outro lado, como $x_{0} \in \bar{H}$ para qualquer $r>0$ existe algum $x^{*} \in H$ tal que $\left\|x^{*}-x_{0}\right\|<r$ e sabendo que $H$ é invariante temos que $\varphi\left(t, x^{*}\right) \in H$ para todo $t \in \mathbb{R}$, em particular $\varphi\left(T, x^{*}\right) \in H$. Logo, tomando $z^{*}=\varphi\left(T, x^{*}\right)$ temos que $z^{*} \in H$ e $\left\|z^{*}-\varphi\left(T, x_{0}\right)\right\|<\varepsilon$ como queríamos demonstrar.

Combinando o resultado do teorema anterior com a invariância da região de estabilidade obtemos o seguinte corolário:

Corolário 3.2.1. Seja $H$ um conjunto atrativo do sistema (2.1). Então $\overline{A(H)}$ é um conjunto invariante.

Uma propriedade importante da região de estabilidade é dada pelo teorema a seguir.

Teorema 3.2.3. (CHIANG; HIRSCH; WU, 1988) Seja H um conjunto atrativo com relação ao sistema (2.1). Então A(H) é um conjunto aberto.

Demonstração ${ }^{2}$. O conjunto $H$ é atrativo, logo, existe constante $\varepsilon>0$ tal que $d\left(x_{0}, H\right)<$ $\varepsilon$ implica que $d\left(\varphi\left(t, x_{0}\right), H\right) \longrightarrow 0$. quando $t \longrightarrow+\infty$. Seja $a \in A(H)$. Então, $d(\varphi(t, a), H) \longrightarrow 0$ quando $t \longrightarrow+\infty$. Logo, existe tempo $T>0$ tal que $d(\varphi(T, a), H)<$ $\frac{\varepsilon}{2}$. Da continuidade das soluções com relação às condições iniciais, dado $\varepsilon>0$ existe $\delta>0$ tal que $d(\varphi(T, a), \varphi(T, b))<\frac{\varepsilon}{2}$ sempre que $d(a, b)<\delta$. Mas $d(\varphi(T, b), H) \leqslant$ $d(\varphi(T, b), \varphi(T, a))+d(\varphi(T, a), H)<\frac{\varepsilon}{2}+\frac{\varepsilon}{2}=\varepsilon$. Portanto, $d(\varphi(s, \varphi(T, b)), H) \longrightarrow 0$ quando $s \longrightarrow+\infty$. Utilizando a propriedade que $\varphi\left(t_{1}+t_{2}, x\right)=\varphi\left(t_{1}, \varphi\left(t_{2}, x\right)\right)$ para todo $x \in \mathbb{R}^{n}$ e $t_{1}, t_{2} \in \mathbb{R}$, temos que $\varphi(t, b)=\varphi(t-T, \varphi(T, b))=\varphi(s, \varphi(T, b))$ onde $s=t-T$. Portanto,

$$
\lim _{t \longrightarrow+\infty} d(\varphi(t, b), H)=\lim _{s \longrightarrow+\infty} d(\varphi(s, \varphi(T, b)), H)=0
$$

sempre que $d(a, b)<\delta$. Consequentemente existe $\delta>0$ tal que todo ponto $b$ satisfazendo $d(a, b)<\delta$ pertence a $A(H)$, isto é, a bola aberta de centro em $a$ e raio $\delta, B(a ; \delta)$ está contida em $A(H)$. Portanto, $A(H)$ é um conjunto aberto.

Discutiremos nos próximos resultados, a conexidade da região de estabilidade. Retornando ao Exemplo 3.1.2, observe que o conjunto $H=\{(-1 ; 0),(1 ; 0)\}$ é um conjunto atrativo do sistema (3.2) e a região de estabilidade de $H=\{(-1 ; 0),(1 ; 0)\}$ é o conjunto $\mathbb{R}^{2}-\left\{(x, y) \in \mathbb{R}^{2}: x=0\right\}$, ou seja, o $\mathbb{R}^{2}$ menos o eixo-y, que não é conexo por caminho, ver Figura 3.4. Porém, qualquer vizinhança atrativa de $H=\{(-1 ; 0),(1 ; 0)\}$ é da forma $U=U_{1} \cup U_{2}$ onde $U_{1}$ e $U_{2}$ são vizinhanças atrativas de $(-1 ; 0)$ e $(1 ; 0)$, respectivamente e $U_{1} \cap U_{2}=\emptyset$.

O conjunto atrativo $H=\{(-1 ; 0),(1 ; 0)\}$ do sistema (3.2) é exemplo de um conjunto atrativo onde a região de estabilidade não é conexa por caminho. Porém, o fato interessante neste exemplo, é que qualquer vizinhança atrativa de $H$ também não é conexa por

\footnotetext{
${ }^{2}$ A demonstração foi retirada do trabalho de Alberto (2006).
} 


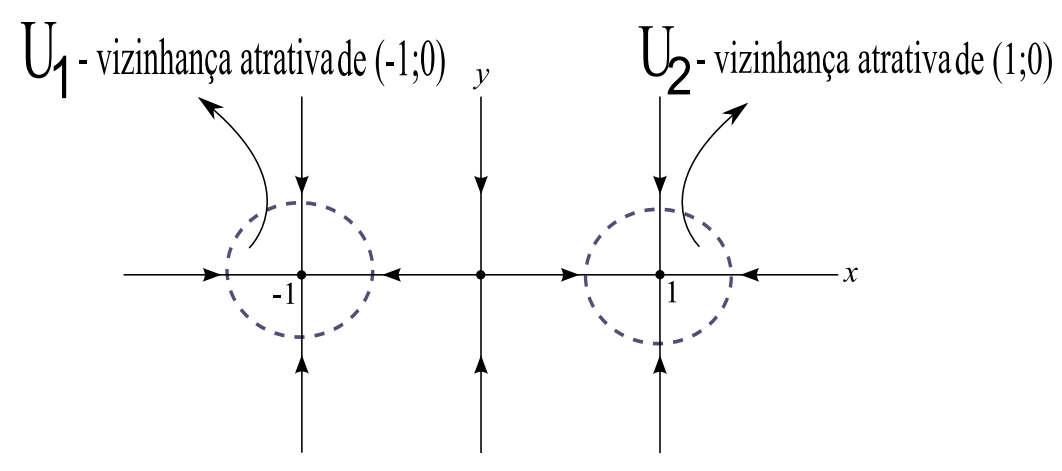

Figura 3.4: Vizinhança atrativa $U=U_{1} \cup U_{2}$ do conjunto atrativo $H=\{(-1 ; 0),(1 ; 0)\}$ do sistema (3.2).

caminho. Por outro lado, o teorema a seguir mostra que a existência de pelo menos uma vizinhança atrativa conexa por caminho de um conjunto atrativo é uma condição suficiente para garantir que a região de estabilidade seja conexa por caminho.

Teorema 3.2.4. Seja $H$ um conjunto atrativo do sistema (2.1). Se H possui pelo menos uma vizinhança atrativa conexa por caminho então $A(H)$ é conexa por caminho.

Demonstração. Seja $U$ uma vizinhança atrativa conexa por caminho de $H$. Do fato que $U$ é uma vizinhança atrativa de $H$, temos que $U \subset A(H)$. Considere $x_{0}, x_{1} \in A(H)$. Como $d\left(\varphi\left(t, x_{0}\right), H\right) \longrightarrow 0$ e $d\left(\varphi\left(t, x_{1}\right), H\right) \longrightarrow 0$ quando $t \longrightarrow+\infty$, podemos afirmar que existem $T_{0}, T_{1}>0$ tal $p_{0}=\varphi\left(T_{0}, x_{0}\right) \in U$ e $p_{1}=\varphi\left(T_{1}, x_{1}\right) \in U$. Como por hipótese $U$ é conexa por caminho existe então um caminho ligando os pontos $p_{0}$ e $p_{1}$ em $U$. Usando a transitividade da conexidade por caminho podemos afirmar que existe um caminho conectando os pontos $x_{0}$ ao ponto $x_{1}$ em $A(H)$. Portanto, pela arbitrariedade dos pontos $x_{0}$ e $x_{1}$ temos que $A(H)$ é conexa por caminho.

A Figura 3.5 ilustra a demonstração do Teorema 3.2.4.

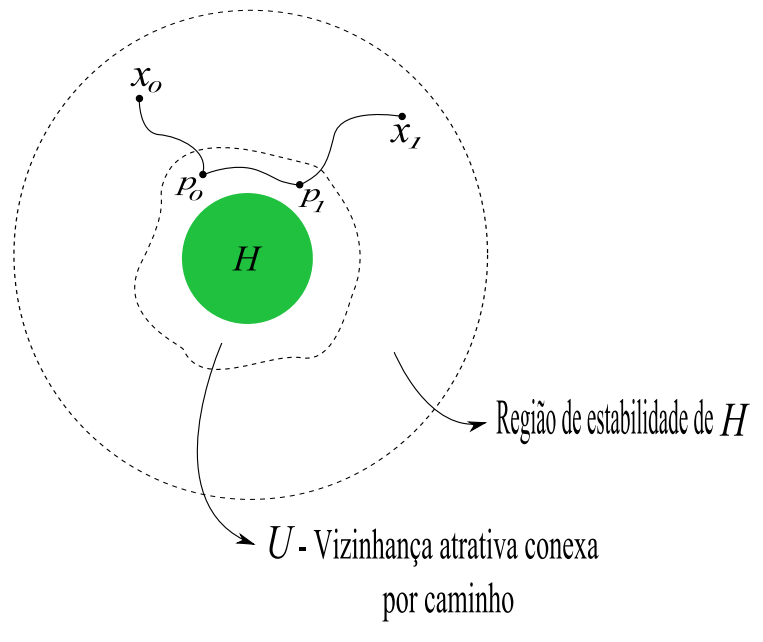

Figura 3.5: Conexidade por caminho da região de estabilidade. 
Corolário 3.2.2. Seja $x^{*}$ um ponto de equilíbrio atrativo de (2.1). Então A(x*) é conexa por caminho.

Demonstração. É uma consequência direta do fato de que todo ponto de equilíbrio atrativo admite uma vizinhança atrativa conexa por caminho e do teorema anterior.

O teorema seguinte prova que a região de estabilidade de um ponto de equilíbrio atrativo é contrátil, sua demonstração pode ser encontrada em (ZABORSZKY et al., 1988), porém verificaremos posteriormente que tal teorema não pode ser generalizado para conjuntos atrativos.

Teorema 3.2.5. (ZABORSZKY et al., 1988) Seja $x_{s}$ um ponto de equilíbrio assintoticamente estável de (2.1). Então a região de estabilidade $A\left(x_{s}\right)$ é contrátil.

Demonstração. Considere a aplicação $H:[0,1] \times A\left(x_{S}\right) \longrightarrow A\left(x_{s}\right)$ definida por

$$
H(t, x)=\left\{\begin{array}{l}
\varphi\left(\operatorname{tg}\left(\frac{\pi t}{2}\right), x\right), \text { se }(t, x) \in[0,1) \times A\left(x_{s}\right) \\
x_{s}, \operatorname{se}(t, x) \in\{1\} \times A\left(x_{s}\right)
\end{array}\right.
$$

A notação $t g$ denota a aplicação tangente. Observe que pela definição da aplicação $H$ temos que $H(0, x)=\varphi(0, x)=x=I d(x)$ e $H(1, x)=x_{s}=g(x)$, onde $I d: A\left(x_{s}\right) \longrightarrow A\left(x_{s}\right)$ é a aplicação identidade e $g: A\left(x_{s}\right) \longrightarrow A\left(x_{s}\right)$ dada por $g(x)=x_{s}$ é uma aplicação constante. Mostraremos agora que $H$ é uma aplicação contínua. Para isso, basta mostrar que $\lim _{(t, x) \rightarrow(1, z)} H(t, x)=H(1, z) \forall z \in A\left(x_{s}\right)$. Sendo assim, note que

$$
t \longrightarrow 1 \Longrightarrow \frac{\pi t}{2} \longrightarrow \frac{\pi}{2} \Longrightarrow \operatorname{tg}\left(\frac{\pi t}{2}\right) \longrightarrow+\infty
$$

Portanto, $\lim _{(t, x) \rightarrow(1, z)} H(t, x)=\lim _{(t, x) \rightarrow(1, z)} \varphi\left(t g\left(\frac{\pi t}{2}\right), x\right)=x_{s}=H(1, z) \forall z \in A\left(x_{s}\right)$, e consequentemente $H$ é uma homotopia entre a aplicação identidade $I d: A\left(x_{s}\right) \longrightarrow A\left(x_{s}\right)$ e a aplicação constante $g: A\left(x_{s}\right) \longrightarrow A\left(x_{s}\right)$, o que mostra que $A\left(x_{s}\right)$ é contrátil.

No Exemplo 3.1.1, se considerarmos apenas o ciclo limite estável $H_{1}$, ele ainda é um conjunto atrativo, visto que, é fechado, invariante e a vizinhança $U_{1}$ de $H_{1}$ da Figura 3.1 é uma vizinhança atrativa de $H_{1}$. A região de estabilidade de $H_{1}$ neste caso é o $\mathbb{R}^{2}-\{(0,0)\}$ que por sua vez não é um conjunto contrátil. Sendo assim, o Teorema 3.2.5 não pode ser generalizado para conjuntos atrativos em geral.

Vimos na seção anterior que a união de dois conjuntos atrativos é ainda um conjunto atrativo. Assim, a região de estabilidade para um conjunto atrativo que se decompõe como união finita de conjuntos atrativos vai ser explorada nos próximos resultados.

Teorema 3.2.6. Sejam $H \subset \mathbb{R}^{n}$ e $K \subset \mathbb{R}^{n}$ conjuntos atrativos de (2.1) tal que $K \subset H$. Então $A(K) \subset A(H)$. 
Demonstração. Seja $x_{0} \in A(K)$. Então $d\left(\varphi\left(t, x_{0}\right), K\right) \longrightarrow 0$ quando $t \longrightarrow+\infty$, ou seja, dado $\varepsilon>0$ existe $T>0$ tal que $d\left(\varphi\left(t, x_{0}\right), K\right)<\varepsilon$ para todo $t>T$. Como $d\left(\varphi\left(t, x_{0}\right), H\right) \leqslant$ $d\left(\varphi\left(t, x_{0}\right), K\right)$, temos que $d\left(\varphi\left(t, x_{0}\right), H\right) \leqslant d\left(\varphi\left(t, x_{0}\right), K\right)<\varepsilon$ para todo $t>T$, isto é, $d\left(\varphi\left(t, x_{0}\right), H\right) \longrightarrow 0$ quando $t \longrightarrow+\infty$ e consequentemente $x_{0} \in A(H)$.

O próximo resultado garante que se um conjunto atrativo se decompõe como união de conjuntos atrativos $H$ e $K$, então a região de estabilidade da união dos conjuntos atrativos é a união das regiões de estabilidade de $H$ e $K$.

Teorema 3.2.7. Sejam $H \subset \mathbb{R}^{n}$ e $K \subset \mathbb{R}^{n}$ conjuntos atrativos de (2.1). Então $A(H \cup K)=$ $A(H) \cup A(K)$.

Demonstração. Mostraremos primeiramente que $A(H \cup K) \subset A(H) \cup A(K)$. De fato, se $x_{0} \in A(H \cup K)$ então $d\left(\varphi\left(t, x_{0}\right), H \cup K\right) \longrightarrow 0$ quando $t \longrightarrow+\infty$. Como $H$ e $K$ são conjuntos atrativos, existem vizinhanças atrativas $U$ e $V$ de $H$ e $K$, respectivamente. Considere $U \cup V$, que é uma vizinhança atrativa de $H \cup K$. Então existe $T>0$ tal que $\varphi\left(T, x_{0}\right) \in$ $U \cup V$, pois $d\left(\varphi\left(t, x_{0}\right), H \cup K\right) \longrightarrow 0$ quando $t \longrightarrow+\infty$. Logo, $p=\varphi\left(T, x_{0}\right) \in U$ ou $p=$ $\varphi\left(T, x_{0}\right) \in V$. Sem perda de generalidade podemos supor que $p=\varphi\left(T, x_{0}\right) \in U$, ou seja, $d(\varphi(s, p), H) \longrightarrow 0$ quando $s \longrightarrow+\infty$. Tomando $s=t-T$ temos que $s \longrightarrow+\infty$ quando $t \longrightarrow+\infty$. Portanto, usando a propriedade que $\varphi\left(t_{1}+t_{2}, x\right)=\varphi\left(t_{1}, \varphi\left(t_{2}, x\right)\right)$ para todo $x \in \mathbb{R}^{n}$ e $t_{1}, t_{2} \in \mathbb{R}$ temos que, $d\left(\varphi\left(t, x_{0}\right), H\right)=d\left(\varphi\left(s+T, x_{0}\right), H\right)=d\left(\varphi\left(s, \varphi\left(T, x_{0}\right)\right), H\right)=$ $d(\varphi(s, p), H) \longrightarrow 0$ quando $t \longrightarrow+\infty$, ou seja, $x_{0} \in A(H)$ e consequentemente $A(H \cup$ $K) \subset A(H) \cup A(K)$. A inclusão $A(H) \cup A(K) \subset A(H \cup K)$ segue do Teorema 3.2.6.

Como consequência imediata do teorema anterior temos o seguinte resultado para união finita de conjuntos atrativos.

Corolário 3.2.3. Sejam $H_{1}, H_{2}, \ldots, H_{n} \subset \mathbb{R}^{n}$ conjuntos atrativos de (2.1). Então $A\left(H_{1} \cup\right.$ $\left.H_{2} \cup \ldots \cup H_{n}\right)=A\left(H_{1}\right) \cup A\left(H_{2}\right) \cup \ldots \cup A\left(H_{n}\right)$.

O teorema a seguir mostra que a interseção da região de estabilidade de conjuntos atrativos disjuntos é vazia.

Teorema 3.2.8. Sejam $H$ e $K$ conjuntos atrativos de (2.1) tal que $H \cap K=\emptyset$. Então $A(H) \cap A(K)=\emptyset$.

Demonstração. Como $H$ e $K$ são conjuntos disjuntos e fechados e o $\mathbb{R}^{n}$ é um espaço normal, podemos afirmar pelo Teorema 2.1.6 que existem abertos disjuntos $U$ e $V$ contendo $H$ e $K$, respectivamente. Seja $x_{0} \in A(H)$. Então $d\left(\varphi\left(t, x_{0}\right), H\right) \longrightarrow 0$ quando $t \longrightarrow+\infty$. Como $H \subset U$, existe $T \in \mathbb{R}$ tal que $\varphi\left(t, x_{0}\right) \in U$ para todo $t>T$. Sabendo que $U \cap V=\emptyset$, podemos afirmar que $\varphi\left(t, x_{0}\right) \notin V$ para todo $t>T$, ou seja, $\varphi\left(t, x_{0}\right)$ não se aproxima de $K$ quando $t \longrightarrow+\infty$ e consequentemente $x_{0} \notin A(K)$, o que mostra que $A(H) \cap A(K)=\emptyset$. 
Exploraremos nos próximos resultados propriedades da fronteira da região de estabilidade. Vale lembrar que algumas das propriedades como a invariância serão fundamentais para o desenvolvimento da caracterização da fronteira da região de estabilidade que será desenvolvida no Capítulo 5.

Teorema 3.2.9. Seja $H$ um conjunto invariante de (2.1). Então a fronteira $\partial H$ também é um conjunto invariante.

Demonstração. Seja $H$ um conjunto invariante e $x_{0} \in \partial H$. Queremos provar que $\varphi\left(t, x_{0}\right) \in$ $\partial H$ para todo $t \in \mathbb{R}$. Como $x_{0} \in \partial H$, então dado $\delta>0$ arbitrariamente pequeno, existem $x_{1} \in H$ e $x_{2} \in \mathbb{R}^{n}-H$ tal que $\left\|x_{0}-x_{1}\right\|<\delta$ e $\left\|x_{0}-x_{2}\right\|<\delta$. Além disso, a invariância de $H$ garante que $\varphi\left(t, x_{1}\right) \in H$ e $\varphi\left(t, x_{2}\right) \in \mathbb{R}^{n}-H$ para todo $t \in \mathbb{R}$. Dado $T \in \mathbb{R}$ arbitrário e $\varepsilon>0$ arbitrariamente pequeno, tem-se, da continuidade das soluções com relação às condições iniciais, que existe $\delta>0$ tal que $\left\|x_{1}-x_{0}\right\|<\delta$ e $\left\|x_{2}-x_{0}\right\|<\delta$ implica em $\left\|\varphi\left(t, x_{1}\right)-\varphi\left(t, x_{2}\right)\right\|<\varepsilon \mathrm{e}\left\|\varphi\left(t, x_{2}\right)-\varphi\left(t, x_{0}\right)\right\|<\varepsilon$ para todo $t \in[-T, T]$. Sendo assim, arbitrariamente próximo de $\varphi\left(T, x_{0}\right)$ existem pontos $\varphi\left(T, x_{1}\right) \in H$ e $\varphi\left(T, x_{2}\right) \in \mathbb{R}^{n}-H$. Portanto, $\varphi\left(T, x_{0}\right) \in \bar{H}$ e $\varphi\left(T, x_{0}\right) \in \overline{\mathbb{R}^{n}-H}$. Por definição, $\varphi\left(T, x_{0}\right) \in \partial H$. Como $x_{0} \mathrm{e}$ $T$ foram escolhidos de maneira arbitrária, tem-se, para todo $x_{0} \in \partial H$, que $\varphi\left(t, x_{0}\right) \in \partial H$ para todo $t \in \mathbb{R}$. Logo, $\partial H$ é um conjunto invariante.

Corolário 3.2.4. Seja $H$ um conjunto atrativo de (2.1). Então a fronteira $\partial A(H) d a$ região de estabilidade é um conjunto fechado e invariante.

Demonstração. Por definição, a fronteira de qualquer conjunto é um conjunto fechado. Logo, $\partial A(H)$ é um conjunto fechado. Como a região de estabilidade é um conjunto invariante segue direto do Teorema 3.2.9 que $\partial A(H)$ é um conjunto invariante.

O próximo teorema estuda a dimensão da fronteira da região de estabilidade.

Teorema 3.2.10. (HUREWICZ.; WALLMAN, 1948) Seja H um conjunto atrativo de (2.1). Então $\operatorname{dim} \partial A(H)<n$. Além disso, se a região de estabilidade $A(H)$ não é densa em $\mathbb{R}^{n}$ então a fronteira $\partial A(H)$ tem dimensão $n-1$.

Observação 3.2.1. Uma condição suficiente para que a região de estabilidade não seja densa em $\mathbb{R}^{n}$ é que o sistema (2.1) possua pelo menos dois conjuntos atrativos disjuntos.

Uma caracterização da fronteira da região de estabilidade para conjuntos atrativos que se decompõem como união de conjuntos atrativos disjuntos é dada a seguir. Mas antes precisaremos demonstrar o seguinte lema.

Lema 3.2.1. Sejam $A, B \subset \mathbb{R}^{n}$ abertos e disjuntos. Se $C \subset \mathbb{R}^{n}$ é conexo, $C \cap\left(\mathbb{R}^{n}-A\right) \neq \emptyset$ e $C \cap A \neq \emptyset$ então $C \cap\left(\mathbb{R}^{n}-(A \cup B)\right) \neq \emptyset$. 
Demonstração. Observe que

$$
C=(C \cap A) \cup(C-(C \cap A))
$$

Além disso, $C-(C \cap A)$ não está inteiramente contido em $B$, pois caso contrário teríamos que

$$
C=(C \cap A) \cup(C \cap B),
$$

mas como $A$ e $B$ são abertos em $\mathbb{R}^{n}$ e disjuntos concluiria-se que $C \cap A$ e $C \cap B$ são abertos em $C$ e disjuntos, o que contradiz o fato de $C$ ser conexo. Portanto, existe $x \in C-(C \cap A)$ tal que $x \notin B$, consequentemente $x \in C, x \notin A$ e $x \notin B$, o que demonstra o lema.

O próximo exemplo ilustra um caso onde o Lema 3.2.1 não pode ser aplicado.

Exemplo 3.2.1. Seja $A=(-1,0] \subset \mathbb{R}$ e $B=(0,1) \subset \mathbb{R}$. Observe que o intervalo $C=$ $\left(-\frac{1}{2}, \frac{1}{2}\right)$ é conexo, $C \cap(\mathbb{R}-A) \neq \emptyset$ e $C \cap A \neq \emptyset$, mas $C \cap(\mathbb{R}-(A \cup B))=\emptyset$. Neste exemplo, $A=(-1,0]$ e $B=(0,1)$ são disjuntos, porém o conjunto $A=(-1,0]$ não é aberto em $\mathbb{R}$. Assim, a hipótese de $A$ e $B$ serem abertos é fundamental na demonstração do Lema 3.2.1.

Com o Lema 3.2.1 estamos aptos a demonstrar o seguinte teorema.

Teorema 3.2.11. Sejam $U, V \subset \mathbb{R}^{n}$ conjuntos abertos e disjuntos. Então $\partial(U \cup V)=$ $\partial U \cup \partial V$.

Demonstração. Mostraremos primeiramente que $\partial(U \cup V) \subset \partial U \cup \partial V$. De fato, seja $x \in \partial(U \cup V)$. Então $\forall r>0$ tem-se $B(x ; r) \cap(U \cup V) \neq \emptyset$ e $B(x ; r) \cap\left(\mathbb{R}^{n}-(U \cup V)\right) \neq \emptyset$. Daí segue que $B(x ; r) \cap U \neq \emptyset$ e $B(x ; r) \cap\left(\mathbb{R}^{n}-U\right) \neq \emptyset$ ou $B(x ; r) \cap V \neq \emptyset$ e $B(x ; r) \cap\left(\mathbb{R}^{n}-\right.$ $V) \neq \emptyset$. Logo, $x \in \partial U$ ou $x \in \partial V$, isto é, $x \in \partial U \cup \partial V$. Mostraremos agora que $\partial U \cup \partial V \subset$ $\partial(U \cup V)$. De fato, seja $x \in \partial U \cup \partial V$, sem perda de generalidade podemos assumir que $x \in \partial U$, ou seja, $\forall r>0$ tem-se $B(x ; r) \cap U \neq \emptyset$ e $B(x ; r) \cap\left(\mathbb{R}^{n}-U\right) \neq \emptyset$. Como a bola aberta $B(x ; r)$ é conexa, $U$ e $V$ são abertos e disjuntos, o Lema 3.2.1 nos garante que $B(x ; r) \cap\left(\mathbb{R}^{n}-(U \cup V)\right) \neq \emptyset$. Portanto, $B(x ; r) \cap(U \cup V) \neq \emptyset$ já que $B(x ; r) \cap U \neq \emptyset \mathrm{e}$ $B(x ; r) \cap\left(\mathbb{R}^{n}-(U \cup V)\right) \neq \emptyset$, isto é, $x \in \partial(U \cup V)$, como queríamos demonstrar.

Corolário 3.2.5. Sejam $H$ e $K$ conjuntos atrativos e disjuntos de (2.1). Então $\partial A(H \cup K)=\partial A(H) \cup \partial A(K)$.

Demonstração. Já vimos que $A(H)$ e $A(K)$ são conjuntos abertos pelo Teorema 3.2.3. O Teorema 3.2.8 afirma que se $H$ e $K$ forem disjuntos então $A(H)$ e $A(K)$ também são disjuntos. Portanto, pelo Teorema 3.2.11 podemos afirmar que

$$
\partial A(H) \cup \partial A(K)=\partial(A(H) \cup A(K)),
$$


mas pelo Teorema 3.2.7 temos que $A(H) \cup A(K)=A(H \cup K)$, assim

$$
\partial A(H) \cup \partial A(K)=\partial(A(H) \cup A(K))=\partial A(H \cup K),
$$

como queríamos demonstrar. 



\section{CARACTERIZAÇÃO DA FRONTEIRA DA REGIÃO DE ESTABILIDADE}

A caracterização da fronteira da região de estabilidade de um ponto de equilíbrio assintoticamente estável foi extensivamente estudada para a classe de sistemas dinâmicos autônomos não lineares (2.1) onde os conjuntos limites na fronteira são restritos a pontos de equilíbrio (CHIANG; HIRSCH; WU, 1988). Neste capítulo, faremos uma revisão sobre a teoria existente dessa caracterização. Na Seção 4.1, exibiremos condições necessárias e suficientes para um ponto de equilíbrio hiperbólico pertencer à fronteira da região de estabilidade. Além disso, veremos que a fronteira da região de estabilidade é a união das variedades estáveis dos pontos de equilíbrio que pertencem à fronteira. $\mathrm{Na}$ Seção 4.2, estudaremos o número dos pontos de equilíbrio na fronteira da região de estabilidade e exibiremos condições suficientes para a fronteira da região de estabilidade ser ilimitada.

\subsection{Pontos de equilíbrio na fronteira da região de estabilidade}

A caracterização da fronteira da região de estabilidade está intimamente relacionada com os pontos de equilíbrios instáveis que pertencem à fronteira. Exibiremos condições para que um ponto de equilíbrio hiperbólico esteja na fronteira da região de estabilidade de um ponto de equilíbrio assintoticamente estável. Este teorema é um passo chave para a caracterização da região de estabilidade.

Teorema 4.1.1. (Caracterização de um ponto de equilíbrio na fronteira da região de estabilidade)(CHIANG; HIRSCH; WU, 1988) Seja $x^{s}$ um ponto de equilíbrio assintoticamente estável de (2.1) e $x^{*} \neq x^{s}$ um ponto de equilíbrio hiperbólico de (2.1). Então:

(i) $x^{*} \in \partial A\left(x^{s}\right)$ se, e somente se, $\left\{W^{u}\left(x^{*}\right)-x^{*}\right\} \cap \overline{A\left(x^{s}\right)} \neq \emptyset$.

(ii) se $x^{*}$ é um ponto de equilíbrio do tipo $k$, com $k<n$, então $x^{*} \in \partial A\left(x^{s}\right)$ se, e somente se $\left\{W^{s}\left(x^{*}\right)-x^{*}\right\} \cap \partial A\left(x^{s}\right) \neq \emptyset$.

O teorema anterior exibe condições necessárias e suficientes, em termos das va-riedades estáveis e instáveis dos equilíbrios para garantir que um ponto de equilíbrio hiperbólico 
pertença à fronteira da região de estabilidade. O Teorema 4.1.1 pode ser melhorado sob três suposições adicionais. A seguir formalizaremos estas suposições.

Seja $x^{s}$ um ponto de equilíbrio de equilíbrio assintoticamente estável de (2.1) e considere as seguintes suposições sobre o campo vetorial.

(A1) Todos pontos de equilíbrio em $\partial A\left(x^{S}\right)$ são hiperbólicos.

(A2) As variedades estáveis e instáveis dos pontos de equilíbrio em $\partial A\left(x^{S}\right)$ satisfazem a condição de transversalidade.

(A3) Toda trajetória em $\partial A\left(x^{s}\right)$ se aproxima de um ponto de equilíbrio quando $t \rightarrow+\infty$.

As suposições $(A 1)$ e $(A 2)$ são propriedades genéricas de sistemas dinâmicos na forma de (2.1) conforme análise da Seção 2.4 e portanto não precisam ser verificadas na prática. Apesar destas condições serem genéricas, elas não são fáceis de serem verificadas. A suposição (A3) não é uma propriedade genérica, mas a existência de uma função energia (CHIANG; HIRSCH; WU, 1988) é suficiente para que seja satisfeita.

O teorema a seguir explora as suposições $(A 1)-(A 3)$ para oferecer condições, em termos das variedades estável e instável, para garantir que um ponto de equilíbrio hiperbólico pertença à fronteira da região de estabilidade. Este teorema oferece uma maneira computacional para verificar se um ponto de equilíbrio hiperbólico pertence à fronteira da região de estabilidade.

Teorema 4.1.2. (Caracterização adicional de um ponto de equilíbrio na fronteira da região de estabilidade)(CHIANG; HIRSCH; WU, 1988) Seja $x^{s}$ um ponto de equilíbrio assintoticamente estável de (2.1) e $A\left(x^{S}\right)$ sua correspondente região de estabilidade. Se as suposições $(A 1)-(A 3)$ são satisfeitas, então:

(i) o ponto de equilíbrio $x^{*} \in \partial A\left(x^{s}\right)$ se, e somente se $W^{u}\left(x^{*}\right) \cap A\left(x^{s}\right) \neq \emptyset$.

(ii) o ponto de equilíbrio $x^{*} \in \partial A\left(x^{s}\right)$ se, e somente se $W^{s}\left(x^{*}\right) \subseteq \partial A\left(x^{s}\right)$.

O próximo teorema caracteriza a fronteira da região de estabilidade para uma classe ampla de sistemas dinâmicos autônomos não lineares (2.1) cuja fronteira da região de estabilidade é não vazia. Tal teorema afirma que se as suposições $(A 1)-(A 3)$ são satisfeitas, então a fronteira da região de estabilidade é a união das variedades estáveis dos pontos de equilíbrio que pertencem à fronteira.

Teorema 4.1.3. (Caracterização da fronteira da região de estabilidade)(CHIANG; HIRSCH; $W U, 1988)$ Seja $x^{s}$ um ponto de equilíbrio assintoticamente estável de (2.1) e A(x $\left.x^{s}\right)$ sua correspondente região de estabilidade. Se as suposições $(A 1)-(A 3)$ são satisfeitas, então

$$
\partial A\left(x^{s}\right)=\bigcup_{i} W^{s}\left(x_{i}\right)
$$

onde $x_{i}, i=1,2, \ldots$ são os pontos de equilíbrio em $\partial A\left(x^{s}\right)$. 
Apresentaremos a seguir dois exemplos de sistemas onde o teorema anterior não pode ser aplicado em consequência de umas das suposições $(A 1)-(A 3)$ serem violadas. $\mathrm{O}$ intuito de colocar estes exemplos aqui é para observamos a importância das suposições $(A 1)-(A 3)$ na caracterização da fronteira da região de estabilidade dada pelo Teorema 4.1.3.

Exemplo 4.1.1. Considere o sistema

$$
\begin{aligned}
& \dot{x}=\quad x^{2}-x \\
& \dot{y}=x y-y+x(y+1)
\end{aligned}
$$

onde $(x ; y) \in \mathbb{R}^{2}$.

A Figura 4.1 mostra o retrato de fase para o sistema (4.1). O sistema (4.1) possui dois pontos de equilíbrio, são eles, $(0 ; 0)$ um ponto de equilíbrio assintoticamente estável e $(1 ;-1)$ um ponto de equilíbrio hiperbólico do tipo 2 . A fronteira da região de estabilidade do ponto de equilíbrio assintoticamente estável $(0 ; 0)$ não é formada pela variedade estável do ponto de equilíbrio $(1 ;-1)$ que pertence a sua fronteira. O Teorema 4.1 .3 não pode ser aplicado, pois apesar das suposições $(A 1)$ e $(A 2)$ serem satisfeitas, a hipótese $(A 3)$ é violada.

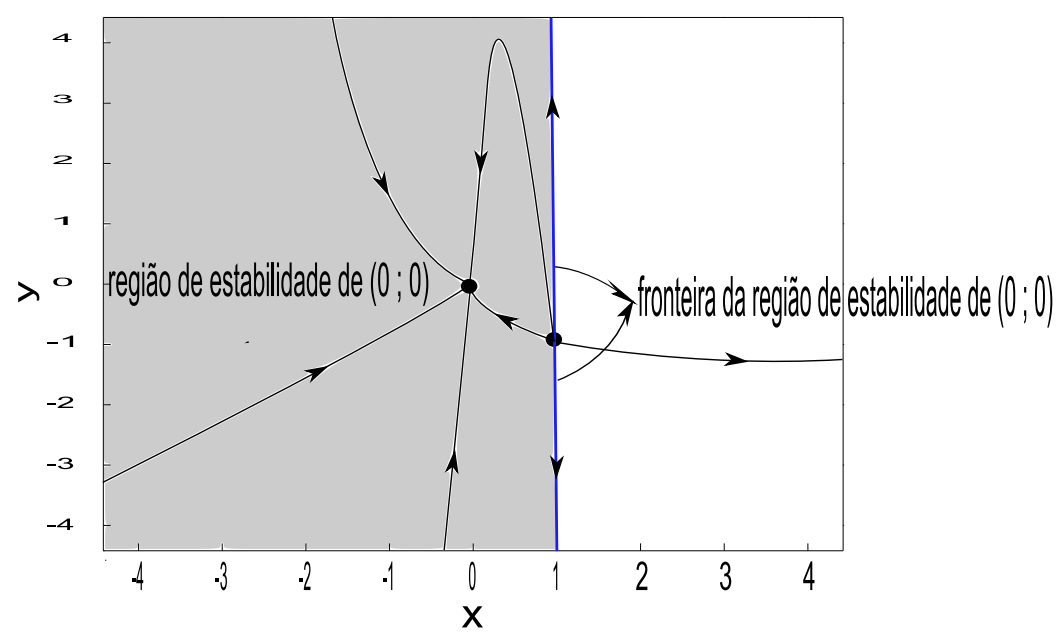

Figura 4.1: Retrato de fase do sistema (4.1).

Exemplo 4.1.2. Considere o sistema

$$
\begin{aligned}
& \dot{x}=\quad y \\
& \dot{y}=0.5-0.7 \sin (x)-0.518265 y
\end{aligned}
$$

onde $(x ; y) \in \mathbb{R}^{2}$.

A Figura 4.2 mostra o retrato de fase para o sistema (4.2). O sistema (4.2) possui três pontos de equilíbrio, são eles, $(0,7956 ; 0)$ um ponto de equilíbrio assintoticamente 
estável, $(-3,9372 ; 0)$ um ponto de equilíbrio hiperbólico do tipo 1 e $(2,346 ; 0)$ um ponto de equilíbrio hiperbólico do tipo 1. A fronteira da região de estabilidade do ponto de equilíbrio assintoticamente estável $(0,7956 ; 0)$ não é formada pela união das variedades estáveis dos pontos de equilíbrio $(-3,9372 ; 0)$ e $(2,346 ; 0)$ que pertencem a sua fronteira. O Teorema 4.1 .3 não pode ser aplicado, pois apesar das suposições $(A 1)$ e $(A 3)$ serem satisfeitas, a hipótese $(A 2)$ é violada, já que a variedade instável do ponto de equilíbrio $(-3,9372 ; 0)$ não intersepta transversalmente a variedade estável do ponto de equilíbrio $(2,346 ; 0)$, ver Figura 4.2 .

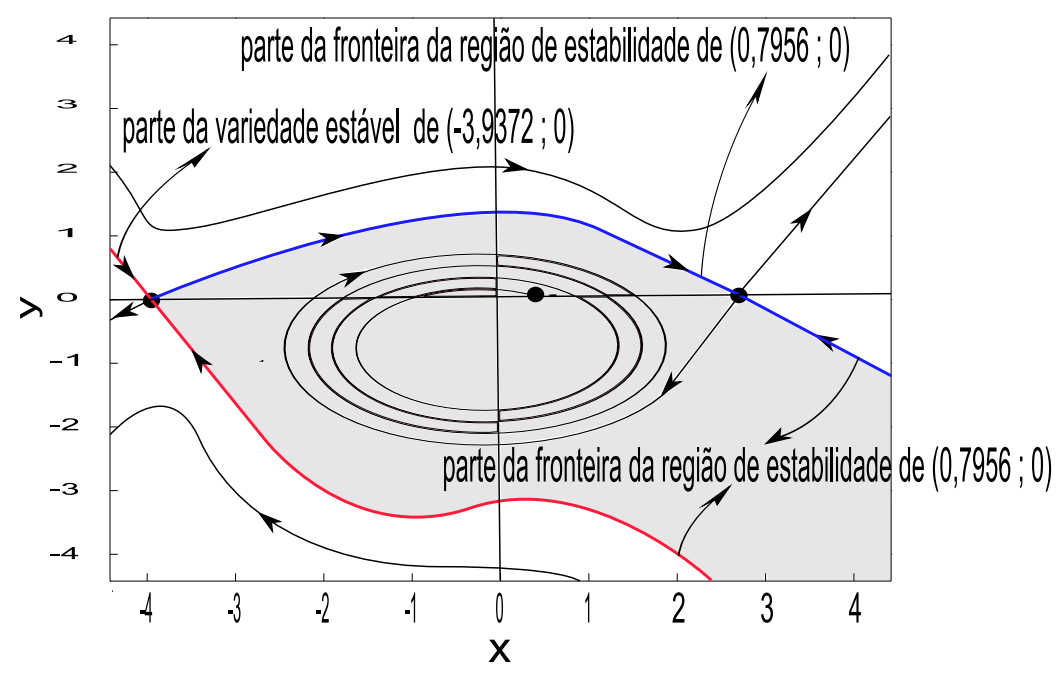

Figura 4.2: Retrato de fase do sistema (4.2).

Finalizando esta seção, exibiremos um exemplo de um sistema dinâmico autônomo não linear onde o Teorema 4.1.3 pode ser aplicado.

Exemplo 4.1.3. Considere o sistema

$$
\begin{aligned}
& \dot{x}=-2 x+x y \\
& \dot{y}=-y+x y
\end{aligned}
$$

onde $(x ; y) \in \mathbb{R}^{2}$.

A Figura 4.3 mostra o retrato de fase para o sistema (4.3). O sistema (4.3) possui dois pontos de equilíbrio, são eles, $(0 ; 0)$ um ponto de equilíbrio assintoticamente estável e $(1 ; 2)$ um ponto de equilíbrio hiperbólico do tipo 1 . As suposições $(A 1)-(A 3)$ são satisfeitas e consequentemente pelo Teorema 4.1 .3 a fronteira da região de estabilidade de $(0 ; 0)$ é a variedade estável do ponto de equilíbrio $(1 ; 2)$, ver Figura 4.3. 


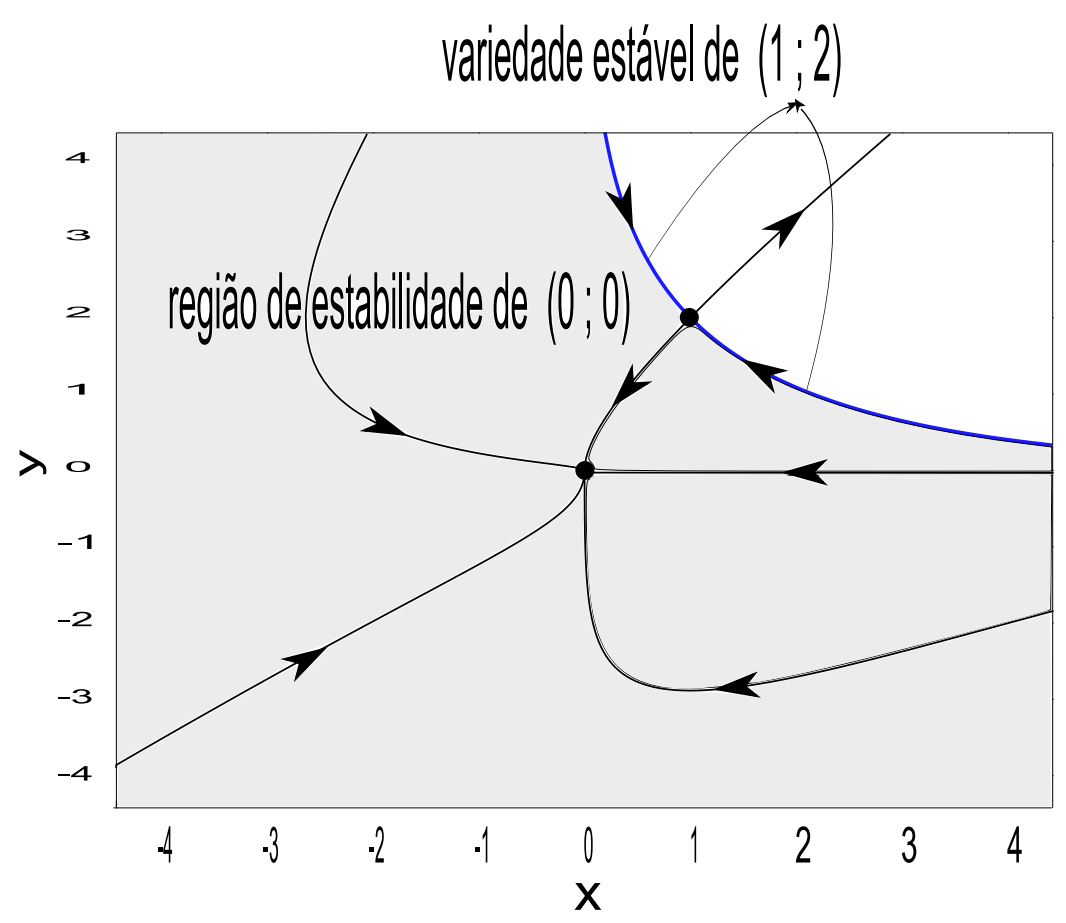

Figura 4.3: Retrato de fase do sistema (4.3).

\subsection{Estrutura dos pontos de equilíbrio na fronteira da região de es- tabilidade}

O primeiro teorema desta seção permite concluir sobre o número de pontos de equilíbrio na fronteira da região de estabilidade.

Teorema 4.2.1. (Número de pontos de equilíbrio na fronteira da região de estabilidade)(CHIANG; HIRSCH; WU, 1988) Se a fronteira da região de estabilidade $\partial A\left(x^{S}\right)$ de um ponto de equilíbrio assintoticamente estável $x^{s}$ de (2.1) é uma variedade compacta de classe $C^{1}$ e todos os pontos de equilíbrio em $\partial A\left(x^{s}\right)$ são hiperbólicos, então o número dos pontos de equilíbrio em $\partial A\left(x^{s}\right)$ é par.

O Teorema 4.2.1 continua válido se em vez de hiperbolicidade, admitirmos somente que todo ponto de equilíbrio é não-degenerado no sentido de que a matriz Jacobiana correspondente ao campo vetorial calculado nos pontos de equilíbrio é invertível.

$\mathrm{O}$ teorema a seguir oferece um resultado interessante a respeito da estrutura dos pontos de equilíbrio na fronteira da região de estabilidade. Além disso, ele apresenta uma condição necessária para a existência de certos tipos de pontos de equilíbrio na fronteira da região de estabilidade limitada.

Teorema 4.2.2. (Estrutura dos pontos de equilíbrio na fronteira da região de estabilidade)(CHIANG; HIRSCH; WU, 1988) Seja $x^{s}$ um ponto de equilíbrio assintoticamente estável de (2.1). Se a região de estabilidade $A\left(x^{s}\right)$ não é densa em $\mathbb{R}^{n}$ e as suposições $(A 1)-(A 3)$ são satisfeitas, então $\partial A\left(x^{S}\right)$ deve conter pelo menos um ponto de equilíbrio 
do tipo 1. Além disso, se a região de estabilidade $A\left(x^{s}\right)$ é limitada, então a fronteira da região de estabilidade deve conter pelo menos um ponto de equilíbrio do tipo 1 e um do tipo $n$.

Uma consequência imediata do Teorema 4.2.2 é o corolário a seguir. Tal corolário exibe condições suficientes para a região de estabilidade ser ilimitada.

Corolário 4.2.1. (Condição suficiente para a região de estabilidade ser ilimitada)(CHIANG;

HIRSCH; WU, 1988) Seja $x^{s}$ um ponto de equilíbrio assintoticamente estável de (2.1). Se a região de estabilidade $A\left(x^{s}\right)$ não é densa em $\mathbb{R}^{n}$, as suposições $(A 1)-(A 3)$ são satisfeitas e $\partial A\left(x^{s}\right)$ não contém um ponto de equilíbrio do tipo $n$, então a região de estabilidade $A\left(x^{s}\right)$ é ilimitada. 


\section{CARACTERIZAÇÃO DA FRONTEIRA DA REGIÃO DE ESTABILIDADE NA PRESENÇA DE UM PONTO DE EQUI- LÍBRIO SELA-NÓ DO TIPO ZERO}

No capítulo 4, apresentamos, sob as suposições $(A 1)$ - $(A 3)$, uma caracterização da fronteira da região de estabilidade em termos das variedades estáveis dos pontos de equilíbrio que pertencem à fronteira. Neste capítulo, vamos enfraquecer a suposição $(A 1)$ admitindo a existência de um ponto de equilíbrio não hiperbólico sela-nó do tipo zero na fronteira da região de estabilidade e desenvolver resultados que permitem ainda caracterizar a fronteira da região de estabilidade em termos das variedades estáveis dos pontos de equilíbrio que pertencem à fronteira, incluindo o ponto de equilíbrio não hiperbólico sela-nó do tipo zero. Na Seção 5.1, classificaremos os pontos de equilíbrio sela-nó e exploraremos o comportamento dinâmico local de um tipo específico de equilíbrio sela-nó, a saber ponto de equilíbrio sela-nó do tipo zero. Na Seção 5.2, exibiremos uma versão do $\lambda$-Lema para ponto de equilíbrio sela-nó do tipo zero. Na Seção 5.3, será apresentado um exemplo motivacional que ilustra o resultado principal deste capítulo, que é a caracterização da fronteira da região de estabilidade na presença de um ponto de equilíbrio sela-nó do tipo zero. Na Seção 5.4, exibiremos alguns lemas auxiliares que serão fundamentais na prova dos teoremas da Seção 5.5. Na Seção 5.5, obteremos uma caracterização da fronteira da região de estabi-lidade na presença de um ponto de equilíbrio sela-nó do tipo zero. Por fim, na Seção 5.6, definiremos o conceito de região de estabilidade fraca e exploraremos algumas das suas propriedades.

\subsection{Ponto de equilíbrio sela-nó}

Nesta seção, um tipo específico de ponto de equilíbrio não hiperbólico é estudado, a saber ponto de equilíbrio sela-nó do tipo zero. Em particular, o comportamento dinâmico na vizinhança do equilíbrio é investigado em detalhes incluindo o comportamento assintótico das soluções nas variedades locais invariantes.

Definição 5.1.1. (Ponto de Equilíbrio Sela-Nó) Um ponto de equilíbrio não hiperbólico 
$p \in \mathbb{R}^{n}$ de (2.1) é chamado um ponto de equilíbrio sela-nó se as seguintes condições são satisfeitas:

(SN1) $D_{x} f(p)$ tem um único autovalor simples igual a 0 com $v$ sendo um autovetor à direita e wà esquerda.

(SN2) $w\left(D_{x}^{2} f(p)(v, v)\right) \neq 0$.

Pontos de equilíbrio sela-nó podem ser classificados em tipos de acordo com o número de autovalores de $D_{x} f(p)$ com parte real positiva.

Definição 5.1.2. (Tipo de Equilíbrio Sela-Nó) Um ponto de equilíbrio sela-nó p de (2.1), é chamado um ponto de equilíbrio sela-nó do tipo $k$ se $D_{x} f(p)$ tem $k$ autovalores com parte real positiva e $n-k-1$ com parte real negativa, $k \in \mathbb{N}$.

Neste texto, estudaremos apenas os pontos de equilíbrio sela-nó do tipo zero. Sendo assim, alguns resultados especializados para pontos de equilíbrio sela-nó do tipo zero são desenvolvidos.

Dado $p$ um ponto de equilíbrio sela-nó do tipo zero de (2.1), o Teorema 2.2.8 garante a existência de variedades locais invariantes de $p$ que são as variedades locais central e estável. A seguir descrevemos algumas propriedades dessas variedades. Para maiores detalhes ver (SOTOMAYOR, 1973):

(1) A variedade local central unidimensional $W_{l o c}^{c}(p)$ de $p$ pode ser decomposta em três subvariedades invariantes:

$$
W_{l o c}^{c}(p)=W_{l o c}^{c^{-}}(p) \cup\{p\} \cup W_{l o c}^{c^{+}}(p)
$$

A Figura 5.1 ilustra a divisão de $W_{l o c}^{c}(p)$. Se $q \in W_{l o c}^{c^{-}}(p)$ então $\varphi(t, q) \longrightarrow p$ quando $t \longrightarrow+\infty$. Se $q \in W_{l o c}^{c^{+}}(p)$ então $\varphi(t, q) \longrightarrow p$ quando $t \longrightarrow-\infty$. Além disso, $W_{l o c}^{c^{+}}(p)$ é única enquanto $W_{l o c}^{c^{-}}(p)$ não é. A Figura 5.1 mostra três possíveis escolhas para $W_{l o c}^{c^{-}}(p)$.

(2) A variedade local estável (n-1)-dimensional $W_{l o c}^{s}(p)$ de $p$ é única e se $q \in W_{l o c}^{s}(p)$ então $\varphi(t, q) \longrightarrow p$ quando $t \longrightarrow+\infty$.

(3) Existe uma vizinhança $N$ de $p$ onde o retrato de fase do sistema (2.1) em $N$ é topologicamente equivalente ao retrato de fase da Figura 5.1.

A variedade global estável e instável de um ponto de equilíbrio hiperbólico são definidas estendendo-se as variedades locais estáveis e instáveis através do fluxo como foi visto na Seção 2.2.5. Usualmente, esta técnica para definir as variedades globais não pode ser aplicada para pontos de equilíbrio não hiperbólicos. Entretanto, no caso particular de um ponto de equilíbrio sela-nó do tipo zero $p$, podemos ainda definir a variedade global estável $W^{s}(p)$ e a variedade global central $W^{c}(p)$ estendendo-se a variedade local $W_{l o c}^{s}(p)$ e $W_{l o c}^{c}(p)$ através do fluxo como segue: 


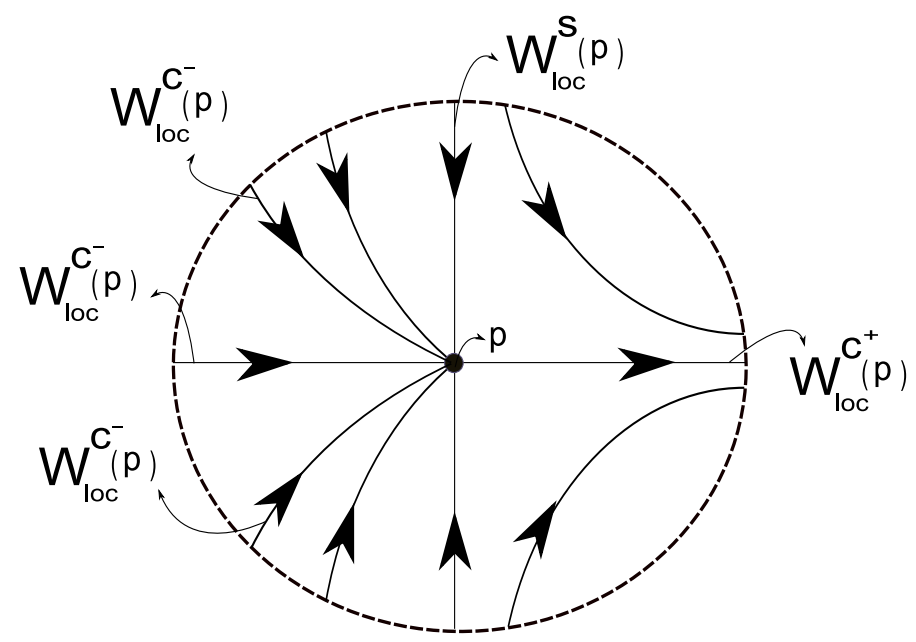

Figura 5.1: As variedades locais $W_{l o c}^{c^{+}}(p)$ e $W_{l o c}^{s}(p)$ são únicas, ao passo que existem infinitas escolhas para $W_{l o c}^{c^{-}}(p)$. Três possíveis escolhas para $W_{l o c}^{c^{-}}(p)$ são indicadas nesta figura.

$$
\begin{gathered}
W^{s}(p):=\bigcup_{t \leqslant 0} \varphi\left(t, W_{l o c}^{s}(p)\right) \\
W^{c}(p):=W^{c^{-}}(p) \bigcup\{p\} \bigcup W^{c^{+}}(p)
\end{gathered}
$$

onde

$$
W^{c^{-}}(p):=\bigcup_{t \leqslant 0} \varphi\left(t, W_{l o c}^{c^{-}}(p)\right)
$$

e

$$
W^{c^{+}}(p):=\bigcup_{t \geqslant 0} \varphi\left(t, W_{l o c}^{c^{+}}(p)\right)
$$

Esta extensão é justificada pela invariância e pelo comportamento assintótico da variedade local estável $W_{l o c}^{s}(p)$ e da variedade local central $W_{l o c}^{c}(p)$ discutidos no item (1) e (2) anteriormente.

Obviamente, se $q \in W^{s}(p)$, então $\varphi(t, q) \longrightarrow p$ quando $t \longrightarrow+\infty$, enquanto $q \in$ $W^{c^{-}}(p)$ implica $\varphi(t, q) \longrightarrow p$ quando $t \longrightarrow+\infty$ e $q \in W^{c^{+}}(p)$ implica $\varphi(t, q) \longrightarrow p$ quando $t \longrightarrow-\infty$.

Observação 5.1.1. A variedade estável $W^{s}(p)$ de um ponto de equilíbrio sela-nó do tipo zero p foi definida de maneira análoga à variedade estável de um ponto de equilíbrio hiperbólico, ver Definição 2.2.15. Porém, $W^{s}(p)$ não pode ser escrita como (2.3). Em geral, as variedades estável e instável de um ponto de equilíbrio não hiperbólico não podem ser escritas como (2.3) $e$ (2.4).

Conhecendo o comportamento dinâmico do sistema próximo a um ponto de equilíbrio sela-nó do tipo zero $p$, qualquer vizinhança $U$ de $p$ pode ser decomposta em subconjuntos $U^{+}$e $U^{-}$, da seguinte maneira: 


$$
\begin{gathered}
U^{-}:=\left\{q \in\left(U-W_{l o c}^{s}(p)\right): \varphi(t, q) \rightarrow p \text { quando } t \rightarrow \infty\right\} \\
U^{+}:=U-U^{-}
\end{gathered}
$$

A Figura 5.2 ilustra o conjunto $U^{-}$.

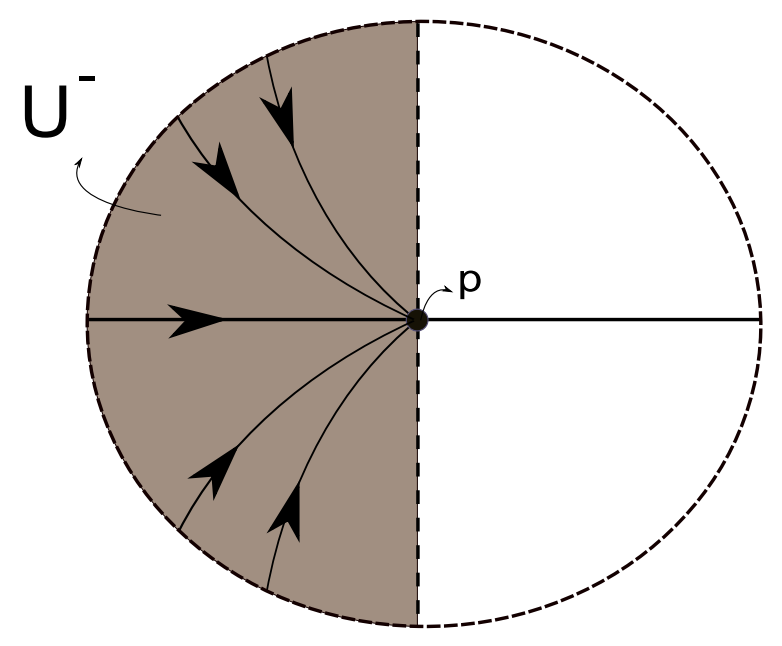

Figura 5.2: Vizinhança $U$ de um ponto de equilíbrio sela-nó do tipo zero $p$ do sistema (2.1). A parte cinza da figura corresponde ao conjunto $U^{-}$.

Observação 5.1.2. O conjunto $U^{-}$é um conjunto aberto em $\mathbb{R}^{n}$.

\section{2 $\lambda$-Lema e equilíbrio sela-nó do tipo zero}

O $\lambda$-lema explora a dinâmica de um sistema não linear nas vizinhanças de um ponto de equilíbrio. Neste trabalho, estaremos interessados na dinâmica nas vizinhanças de um ponto de equilíbrio não hiperbólico sela-nó do tipo zero. Sendo assim, exploraremos nesta seção alguns resultados sobre a versão do $\lambda$-lema para pontos de equilíbrio não hiperbólicos (PALIS, 1969). Esta versão será utilizada no capítulo posterior para obter uma caracterização da fronteira da região de estabilidade na presença de um ponto de equilíbrio sela-nó do tipo zero. Começaremos a seção abordando os conceitos de domínio fundamental e vizinhança fundamental.

Definição 5.2.1. (PALIS, 1969) Sejam $x^{*}$ um ponto de equilíbrio hiperbólico de (2.1)e $B^{u}$ uma vizinhança de $x^{*}$ em $W_{\text {loc }}^{u}\left(x^{*}\right)$ cuja fronteira $\partial B^{u}$ é transversal ao campo $f$ em $W_{l o c}^{u}\left(x^{*}\right)$. A fronteira $\partial B^{u}$ é denominada um domínio fundamental de $W_{l o c}^{u}\left(x^{*}\right)$.

O domínio fundamental $\partial B^{u}$ possui a seguinte propriedade:

$$
\left(W_{l o c}^{u}\left(x^{*}\right)-x^{*}\right)=\bigcup_{t \in \mathbb{R}} \varphi\left(t, \partial B^{u}\right)
$$


Analogamente, define-se domínio fundamental para $W_{l o c}^{s}\left(x^{*}\right)$.

Definição 5.2.2. (PALIS, 1969) Sejam $x^{*}$ um ponto de equilíbrio hiperbólico de (2.1) e $\partial B^{u}$ um domínio fundamental de $W_{l o c}^{u}\left(x^{*}\right)$. Qualquer vizinhança $N^{u}$ de $\partial B^{u}$ disjunta de $W_{l o c}^{s}\left(x^{*}\right)$ é denominada uma vizinhança fundamental de $W_{l o c}^{u}\left(x^{*}\right)$.

Analogamente, define-se vizinhança fundamental para $W_{l o c}^{s}\left(x^{*}\right)$. O resultado a seguir é a versão do $\lambda$-lema para ponto de equilíbrio hiperbólico.

Lema 5.2.1. ( $\lambda$-lema)(PALIS, 1969) Seja $x^{*}$ um ponto de equilíbrio hiperbólico de (2.1). Considere $q \in W_{l o c}^{u}\left(x^{*}\right)$ e $D^{s}$ um disco de dimensão $r=\operatorname{dim} E^{s}\left(x^{*}\right)$, transversal a $W_{l o c}^{u}\left(x^{*}\right)$ em q. Então existe uma vizinhança $U$ de $x^{*}$ em $\mathbb{R}^{n}$ tal que, dado $\varepsilon>0$, existe $t_{-}<0$ tal que $\varphi\left(t, D^{s}\right)$ contém um disco $\varepsilon C^{1}$-próximo a $U \cap W_{\text {loc }}^{s}\left(x^{*}\right)$ para todo $t<t_{-}$.

A Figura 5.3 ilustra o Lema 5.2.1.

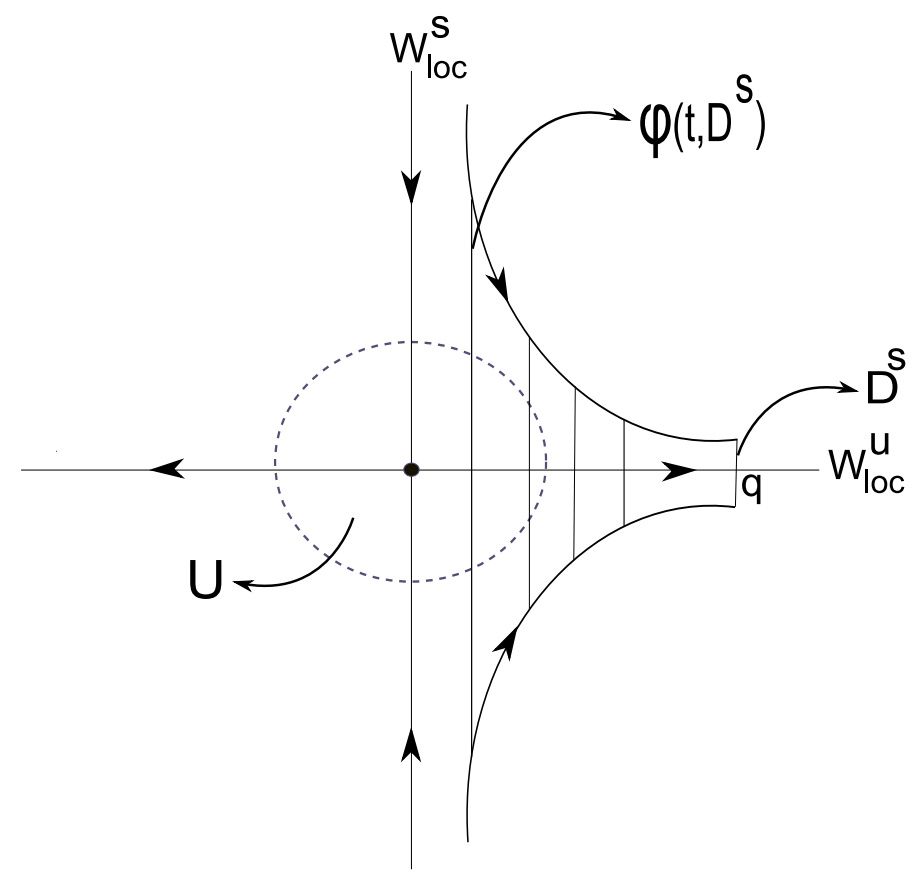

Figura 5.3: Ilustração do Lema 5.2.1.

Como consequência do lema anterior, temos o seguinte corolário.

Corolário 5.2.1. (PALIS, 1969) Seja $x^{*}$ um ponto de equilíbrio hiperbólico de (2.1) e $N^{u}$ uma vizinhança fundamental de $W_{l o c}^{u}\left(x^{*}\right)$. Então existe uma vizinhança $U$ de $x^{*}$ tal que $\cup_{t \leqslant 0} \varphi\left(t, N^{u}\right) \supset U-W_{l o c}^{s}\left(x^{*}\right)$.

A versão do $\lambda$-lema para ponto de equilíbrio sela-nó do tipo zero que será explorada a seguir é uma consequência da versão do $\lambda$-lema para ponto de equilíbrio não hiperbólico que pode ser encontrada em (PALIS, 1969). 
Lema 5.2.2. ( $(\lambda$-lema)(PALIS, 1969) Seja p um ponto de equilíbrio sela-nó do tipo zero de (2.1). Considere $q \in W_{l o c}^{c^{+}}(p)$ e $D^{s}$ um disco de dimensão $n-1$, transversal a $W_{l o c}^{c^{+}}(p)$ em q. Então existe uma vizinhança $U$ de $p$ em $\mathbb{R}^{n}$ tal que, dado $\varepsilon>0$, existe $t_{-}<0$ tal que $\varphi\left(t, D^{s}\right)$ contém um disco $\varepsilon C^{1}$-próximo a $U \cap W_{\text {loc }}^{s}(p)$ para todo $t<t_{-}$.

Como consequência do lema anterior, temos o seguinte corolário.

Corolário 5.2.2. (PALIS, 1969) Seja p um ponto de equilíbrio sela-nó do tipo zero de (2.1). Considere $q \in W_{l o c}^{c^{+}}(p)$ e $D^{s}$ um disco de dimensão $n-1$, transversal a $W_{l o c}^{c^{+}}(p)$ em q. Então existe uma vizinhança $U$ de $p$ tal que $\cup_{t \leqslant 0} \varphi\left(t, D^{s}\right) \supset U^{+}-W_{l o c}^{s}(p)$.

A Figura 5.4 ilustra o Lema 5.2.2 e Corolário 5.2.2.

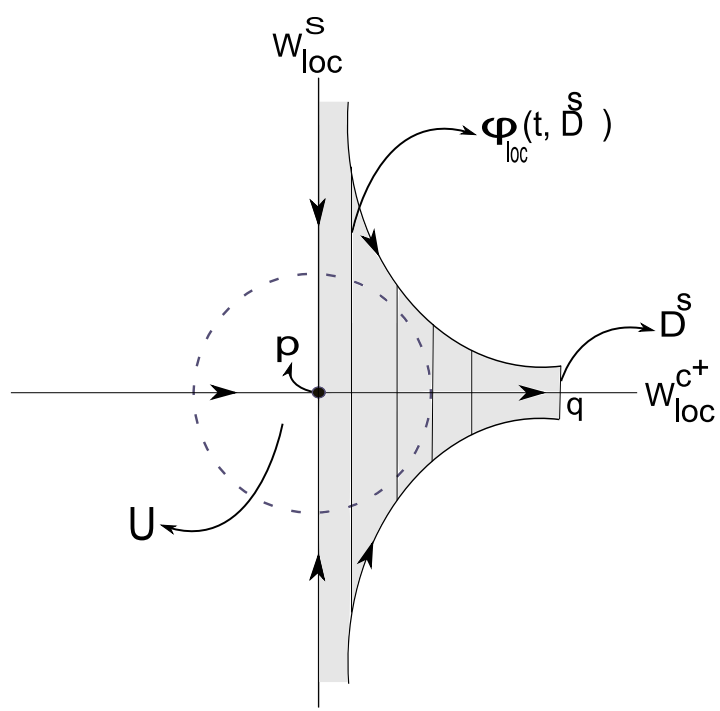

Figura 5.4: Ilustração do Lema 5.2.2 e Corolário 5.2.2. A parte cinza da figura corresponde ao conjunto $\cup_{t \leqslant 0} \varphi\left(t, D^{s}\right)$.

Uma versão dual para o Lema 5.2.2 será exibida a seguir.

Lema 5.2.3. ( $\lambda$-lema)(PALIS, 1969) Seja p um ponto de equilíbrio sela-nó do tipo zero de (2.1). Considere $q \in W_{l o c}^{s}(p)$ e $D^{c}$ um disco unidimensional, transversal a $W_{l o c}^{s}(p)$ em q. Então existe uma vizinhança $U$ de $p$ em $\mathbb{R}^{n}$ tal que, dado $\varepsilon>0$, existe $t_{+}>0$ tal que $\varphi\left(t, D^{c}\right)$ contém um disco $\varepsilon C^{1}$-próximo a $U \cap W_{\text {loc }}^{c^{+}}(p)$ para todo $t>t_{+}$.

A Figura 5.5 ilustra o Lema 5.2.3.

\subsection{Exemplo}

Considere o sistema de equações diferenciais

$$
\begin{aligned}
& \dot{x}=x^{2}+y^{2}-1 \\
& \dot{y}=x^{2}-y-1
\end{aligned}
$$




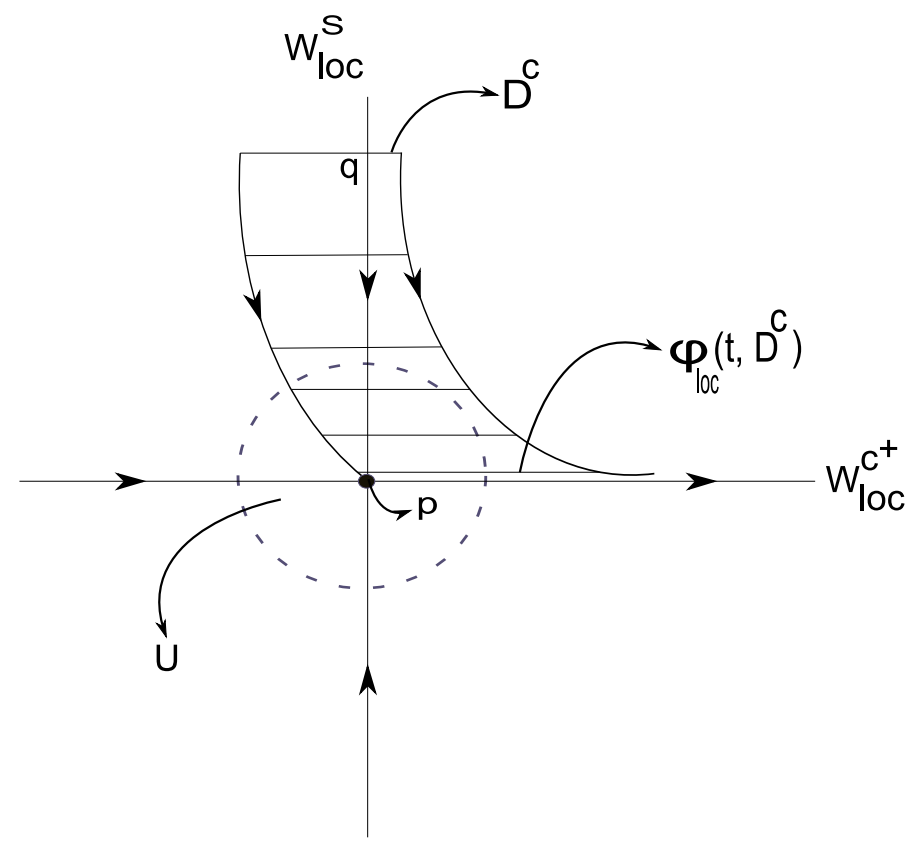

Figura 5.5: Ilustração do Lema 5.2.3.

onde $(x ; y) \in \mathbb{R}^{2}$.

O sistema (5.1) possui, três pontos de equilíbrio; são eles $p=(0 ;-1)$, um ponto de equilíbrio sela-nó do tipo zero, $x^{s}=(-1 ; 0)$, um ponto de equilíbrio hiperbólico assintoticamente estável e $x^{*}=(1 ; 0)$, um ponto de equilíbrio hiperbólico do tipo um. Ambos, o ponto de equilíbrio sela-nó do tipo zero $p=(0 ;-1)$ e o ponto de equilíbrio hiperbólico do tipo um $x^{*}=(1 ; 0)$ pertencem à fronteira da região de estabilidade de $x^{s}=(-1 ; 0)$, ver Figura 5.6. A fronteira da região de estabilidade de $x^{s}=(-1 ; 0)$ é formada pela união da variedade estável do ponto de equilíbrio hiperbólico do tipo um $x^{*}=(1 ; 0)$, a curva azul da Figura 5.6, com a variedade estável do ponto de equilíbrio sela-nó do tipo zero $p=(0 ;-1)$, a curva vermelha da Figura 5.6.

No sistema (5.1), mesmo com a violação da suposição $(A 1)$ devido à presença de um ponto de equilíbrio não hiperbólico sela-nó do tipo zero na fronteira da região de estabilidade de um ponto de equilíbrio assintoticamente estável, a fronteira da região de estabilidade é formada pela união da variedade estável do ponto de equilíbrio hiperbólico que está na fronteira com a variedade estável do ponto de equilíbrio não hiperbólico selanó do tipo zero. Essa caracterização da fronteira da região de estabilidade apresentada pelo sistema (5.1), é o resultado principal a ser mostrado neste capítulo. Para os sistemas dinâmicos autônomos não lineares $\dot{x}=f(x)$ que na fronteira da região de estabilidade, sob condições de transversalidade e a condição $(A 3)$, possuam um ponto de equilíbrio não hiperbólico sela-nó do tipo zero, a fronteira é caracterizada pela união das variedades estáveis dos pontos de equilíbrio que estão não fronteira, incluindo a variedade estável do ponto de equilíbrio não hiperbólico sela-nó do tipo zero. Nas demonstrações dos teoremas principais que serão exibidas na Seção 5.5, para caracterizar a fronteira da região 


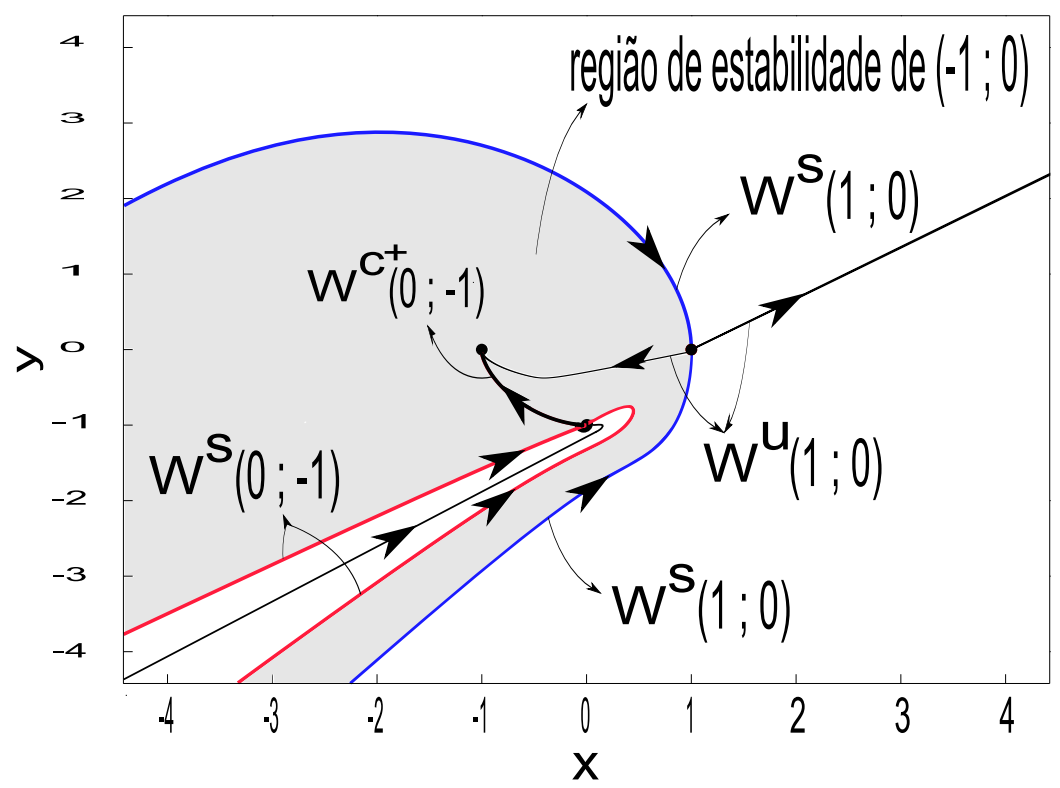

Figura 5.6: O retrato de fase do sistema (5.1). A fronteira da região de estabilidade é constituída pela união da variedade estável do ponto de equilíbrio hiperbólico do tipo um $(1 ; 0)$, a curva em azul, com a variedade estável do ponto de equilíbrio sela-nó do tipo zero $(0 ;-1)$, a curva em vermelho.

de estabilidade na presença de um ponto de equilíbrio sela-nó do tipo zero, utilizaremos alguns lemas que serão demonstrados na próxima seção.

\subsection{Lemas auxiliares}

O primeiro lema desta seção relaciona a dimensão das variedades estáveis e instáveis de pontos de equilíbrio hiperbólicos que se interceptam transversalmente.

Lema 5.4.1. Sejam z e $x^{*}$ dois pontos de equilíbrio hiperbólicos do sistema (2.1). Se $W^{u}\left(x^{*}\right)$ e $W^{s}(z)$ satisfazem a condição de transversalidade $e\left(W^{u}\left(x^{*}\right)-x^{*}\right) \cap\left(W^{s}(z)-\right.$ $z) \neq \emptyset$. Então, $\operatorname{dim} W^{u}\left(x^{*}\right)>\operatorname{dim} W^{u}(z)$.

Demonstração. Como $W^{u}\left(x^{*}\right)$ e $W^{s}(z)$ satisfazem a condição de transversalidade e $W^{u}\left(x^{*}\right) \cap$ $W^{s}(z) \neq \emptyset$, temos pelo Lema 2.3.1 que

$$
\operatorname{dim} W^{u}\left(x^{*}\right)+\operatorname{dim} W^{s}(z)=n+\operatorname{dim}\left(W^{u}\left(x^{*}\right) \cap W^{s}(z)\right) .
$$

Dado $x \in\left(W^{u}\left(x^{*}\right)-x^{*}\right) \cap\left(W^{s}(z)-z\right)$ e como $W^{u}\left(x^{*}\right)$ e $W^{s}(z)$ são conjuntos invariantes, temos que a órbita que passa por $x$ está inteiramente contida em $W^{u}\left(x^{*}\right) \cap W^{s}(z)$, assim podemos afirmar que $\operatorname{dim}\left(W^{u}\left(x^{*}\right) \cap W^{s}(z)\right) \geqslant 1$, isto é,

$$
\operatorname{dim} W^{u}\left(x^{*}\right)+\operatorname{dim} W^{s}(z) \geqslant n+1
$$


ou equivalentemente,

$$
\operatorname{dim} W^{u}\left(x^{*}\right) \geqslant n+1-\operatorname{dim} W^{s}(z) .
$$

Desde que $z$ é um ponto de equilíbrio hiperbólico, temos que

$$
\operatorname{dim} W^{s}(z)+\operatorname{dim} W^{u}(z)=n
$$

assim, a desigualdade anterior nos fornece:

$$
\operatorname{dim} W^{u}\left(x^{*}\right) \geqslant\left(\operatorname{dim} W^{s}(z)+\operatorname{dim} W^{u}(z)+1-\operatorname{dim} W^{s}(z)\right)=\operatorname{dim} W^{u}(z)+1,
$$

isto é,

$$
\operatorname{dim} W^{u}\left(x^{*}\right) \geqslant \operatorname{dim} W^{u}(z)+1>\operatorname{dim} W^{u}(z),
$$

e o lema está provado.

O próximo lema afirma, sob a condição de transversalidade, que a componente instável $W^{c^{+}}(p)$ da variedade central de um ponto de equilíbrio sela-nó do tipo zero $p$ só pode interceptar a variedade estável de um ponto de equilíbrio hiperbólico do tipo zero.

Lema 5.4.2. Sejam p um ponto de equilíbrio sela-nó do tipo zero e $x^{*}$ um ponto de equilíbrio hiperbólico do sistema (2.1). Se $W^{c^{+}}(p)$ e $W^{s}\left(x^{*}\right)$ satisfazem a condição de transversalidade e $W^{c^{+}}(p) \cap W^{s}\left(x^{*}\right) \neq \emptyset$, então $x^{*}$ é do tipo zero.

Demonstração. Como $W^{c^{+}}(p)$ e $W^{s}\left(x^{*}\right)$ satisfazem a condição de transversalidade e $W^{c^{+}}(p) \cap W^{s}\left(x^{*}\right) \neq \emptyset$, temos pelo Lema 2.3.1 que

$$
\operatorname{dim} W^{c^{+}}(p)+\operatorname{dim} W^{s}\left(x^{*}\right)=n+\operatorname{dim}\left(W^{c^{+}}(p) \cap W^{s}\left(x^{*}\right)\right) .
$$

Dado $x \in W^{c^{+}}(p) \cap W^{s}\left(x^{*}\right)$ e como $W^{c^{+}}(p)$ e $W^{s}\left(x^{*}\right)$ são conjuntos invariantes, temos que a órbita que passa por $x$ está inteiramente contida em $W^{c^{+}}(p) \cap W^{s}\left(x^{*}\right)$, assim podemos afirmar que $\operatorname{dim}\left(W^{c^{+}}(p) \cap W^{s}\left(x^{*}\right)\right) \geqslant 1$, isto é,

$$
\operatorname{dim} W^{c^{+}}(p)+\operatorname{dim} W^{s}\left(x^{*}\right) \geqslant n+1
$$

ou equivalentemente,

$$
\operatorname{dim} W^{c^{+}}(p) \geqslant n+1-\operatorname{dim} W^{s}\left(x^{*}\right) .
$$

Desde que $x^{*}$ é um ponto de equilíbrio hiperbólico temos que

$$
\operatorname{dim} W^{s}\left(x^{*}\right)+\operatorname{dim} W^{u}\left(x^{*}\right)=n,
$$

assim a desigualdade anterior nos fornece

$$
\operatorname{dim} W^{c^{+}}(p) \geqslant\left(\operatorname{dim} W^{s}\left(x^{*}\right)+\operatorname{dim} W^{u}\left(x^{*}\right)\right)+1-\operatorname{dim} W^{s}\left(x^{*}\right)=\operatorname{dim} W^{u}\left(x^{*}\right)+1,
$$


isto é,

$$
\operatorname{dim} W^{c^{+}}(p) \geqslant \operatorname{dim} W^{u}\left(x^{*}\right)+1>\operatorname{dim} W^{u}\left(x^{*}\right) .
$$

Desde que $\operatorname{dim} W^{c^{+}}(p)=1$, a desigualdade anterior garante que $\operatorname{dim} W^{u}\left(x^{*}\right)=0$. Sabendo que o tipo de $x^{*}$ é igual a dimensão da variedade instável $W^{u}\left(x^{*}\right)$, o lema está provado.

Usando uma demonstração análoga àquela do lema anterior, temos o seguinte resultado.

Lema 5.4.3. Sejam p um ponto de equilíbrio sela-nó do tipo zero e $x^{*}$ um ponto de equilíbrio hiperbólico do sistema (2.1). Se $W^{s}(p)$ e $W^{u}\left(x^{*}\right)$ satisfazem a condição de transversalidade e $W^{s}(p) \cap W^{u}\left(x^{*}\right) \neq \emptyset$, então $\operatorname{dim} W^{u}\left(x^{*}\right) \geqslant 2$.

O próximo lema conclui, sob a condição de transversalidade, que a interseção da componente instável $W^{c^{+}}(p)$ da variedade central de um ponto de equilíbrio sela-nó do tipo zero $p$ e sua variedade estável é vazia.

Lema 5.4.4. Sejam p um ponto de equilíbrio sela-nó do tipo zero do sistema (2.1). Se $W^{c^{+}}(p)$ e $W^{s}(p)$ satisfazem a condição de transversalidade, então $W^{c^{+}}(p) \cap W^{s}(p)=\emptyset$.

Demonstração. Como $W^{c^{+}}(p)$ e $W^{s}(p)$ satisfazem a condição de transversalidade, temos, pelo Lema 2.3.1, que

$$
\operatorname{dim} W^{c^{+}}(p)+\operatorname{dim} W^{s}(p)-n=\operatorname{dim}\left(W^{c^{+}}(p) \cap W^{s}(p)\right) .
$$

Desde que $\operatorname{dim} W^{c^{+}}(p)=1$ e $\operatorname{dim} W^{s}(p)=n-1$ a desigualdade anterior nos fornece:

$$
\operatorname{dim}\left(W^{c^{+}}(p) \cap W^{s}(p)\right)=1+n-1-n=0
$$

isto é, $\operatorname{dim}\left(W^{c^{+}}(p) \cap W^{s}(p)\right)=0$. Por outro lado, se existe $x \in W^{c^{+}}(p) \cap W^{s}(p)$, então a órbita que passa por $x$ está inteiramente contida em $W^{c^{+}}(p) \cap W^{s}(p)$, assim temos que $\operatorname{dim}\left(W^{c^{+}}(p) \cap W^{s}(p)\right) \geqslant 1$, levando-nos a um absurdo. Portanto, a interseção $W^{c^{+}}(p) \cap$ $W^{s}(p)$ é vazia e o lema está provado.

Finalizando a seção, temos um resultado que mostra que se um ponto de equilíbrio sela-nó do tipo zero $p$ está na fronteira da região de estabilidade de um ponto de equilíbrio assintoticamente estável então a componente estável $W^{c^{-}}(p)$ da variedade central de $p$ tem interseção vazia com a fronteira da região de estabilidade.

Lema 5.4.5. Sejam p um ponto de equilíbrio sela-nó do tipo zero do sistema (2.1) e A(x $\left.x^{s}\right)$ a região de estabilidade de um ponto de equilíbrio assintoticamente estável $x^{s}$. Então $W^{c^{-}}(p) \cap \partial A\left(x^{s}\right)=\emptyset$. 
Demonstração. Suponha pelo contrário a existência de $q \in W^{c^{-}}(p) \cap \partial A\left(x^{s}\right)$. Em particular, $\varphi(t, q) \longrightarrow p$ quando $t \longrightarrow+\infty$. Assim, existe $T>0$ tal que $\varphi(T, q) \in U^{-}$onde $U$ é uma vizinhança de $p$. Como $U^{-}$é um conjunto aberto, existe $\varepsilon>0$, arbitrariamente pequeno, tal que $B(\varphi(T, q), \varepsilon) \subset U^{-}$. Da continuidade das soluções com respeito as condições iniciais, existe $\delta>0$ tal que $d\left(\varphi\left(T, q^{*}\right), \varphi(T, q)\right)<\varepsilon$ para todo $q^{*}, q$ satisfazendo $d\left(q^{*}, q\right)<\delta$. Desde que $q \in \partial A\left(x^{s}\right)$, existe $q^{\prime} \in A\left(x^{s}\right)$ tal que $d\left(q^{\prime}, q\right)<\delta$. Assim, podemos afirmar que $\varphi\left(T, q^{\prime}\right) \in B(\varphi(T, q), \varepsilon) \subset U^{-}$. Explorando a propriedade de convergência do conjunto $U^{-}$e a propriedade do fluxo $\varphi$ obtemos

$$
\varphi\left(t, q^{\prime}\right) \longrightarrow p \text { quando } t \longrightarrow \infty
$$

levando-nos a um absurdo, pois $q^{\prime} \in A\left(x^{s}\right)$. Portanto, $W^{c^{-}}(p) \cap \partial A\left(x^{s}\right)=\emptyset$ e o lema está provado.

\subsection{Ponto de equilíbrio sela-nó do tipo zero na fronteira da região de estabilidade}

Começaremos a seção provando um teorema que oferece uma condição necessária e suficiente para garantir que um ponto de equilíbrio sela-nó do tipo zero esteja na fronteira da região de estabilidade de um ponto de equilíbrio assintoticamente estável.

Teorema 5.5.1. (Ponto de equilíbrio sela-nó do tipo zero na fronteira da região de estabilidade): Sejam $x^{s}$ um ponto de equilíbrio assintoticamente estável de (2.1) e A(x $\left.x^{s}\right)$ sua região de estabilidade. Se pé um ponto de equilíbrio sela-nó do tipo zero de (2.1), então:

$$
p \in \partial A\left(x^{s}\right) \Leftrightarrow W_{l o c}^{c^{+}}(p) \cap \overline{A\left(x^{s}\right)} \neq \emptyset
$$

Demonstração. $(\Longleftarrow)$ Suponha que $W_{l o c}^{c^{+}}(p) \cap \overline{A\left(x^{s}\right)} \neq \emptyset$. Então existe $x \in W_{l o c}^{c^{+}}(p) \cap$ $\overline{A\left(x^{s}\right)}$. Note que, $\varphi(t, x) \longrightarrow p$ quando $t \longrightarrow-\infty$. Por outro lado, o conjunto $\overline{A\left(x^{s}\right)}$ é invariante, assim $\varphi(t, x) \in \overline{A\left(x^{s}\right)}$ para todo $t \leqslant 0$. Como consequência, $p \in \overline{A\left(x^{s}\right)}$. Desde que $p \notin A\left(x^{s}\right)$, temos que $p \in\left(\mathbb{R}^{n}-A\left(x^{s}\right)\right)$. Portanto, $p \in \partial A\left(x^{s}\right)$.

$(\Longrightarrow)$ Suponha que $p \in \partial A\left(x^{s}\right)$. Seja $B(q ; \varepsilon)$ uma bola aberta de raio $\varepsilon>0$ centrada em $q$ para algum $q \in W_{l o c}^{c^{+}}(p)$. Considere um disco $D$ de dimensão $n-1$ contido em $B(q ; \varepsilon)$ e transversal a $W_{l o c}^{c^{+}}(p)$ em $q$. Como consequência do Corolário 5.2.2, existe vizinhança $U$ de $p$ tal que $\cup_{t \leqslant 0} \varphi(t, B(q ; \varepsilon)) \supset\left(U^{+}-W_{l o c}^{s}(p)\right)$. Desde que $p \in \partial A\left(x^{s}\right)$, temos que $U \cap A\left(x^{s}\right) \neq \emptyset$. Por outro lado, $U^{-} \cap A\left(x^{s}\right)=\emptyset$ e $W_{l o c}^{s}(p) \cap A\left(x^{s}\right)=\emptyset$, assim $\left(U^{+}-W_{\text {loc }}^{s}(p)\right) \cap A\left(x^{s}\right) \neq \emptyset$. Logo, existe um ponto $q^{*} \in B(q ; \varepsilon)$ e um tempo $t^{*}$ tal que $\varphi\left(t^{*}, q^{*}\right) \in A\left(x^{s}\right)$. Desde que $A\left(x^{s}\right)$ é invariante, temos que $q^{*} \in A\left(x^{s}\right)$. Como $\varepsilon$ pode ser escolhido arbitrariamente pequeno, podemos encontrar uma sequência de pontos $\left\{q_{i}^{*}\right\}$ com $q_{i}^{*} \in A\left(x^{s}\right)$ para todo $i=1,2, \ldots$ tal que $q_{i}^{*} \longrightarrow q$ quando $i \longrightarrow \infty$, isto é, $q \in \overline{A\left(x^{s}\right)}$. 
Desde que $q \in W_{l o c}^{c^{+}}(p)$, temos que $W_{l o c}^{c^{+}}(p) \cap \overline{A\left(x^{s}\right)} \neq \emptyset$.

Uma versão mais forte do teorema anterior poder ser provada sob algumas suposições adicionais. Sejam $x^{s}$ um ponto de equilíbrio assintoticamente estável e $p$ um ponto de equilíbrio sela-nó do tipo zero de (2.1), e considere as seguintes suposições:

$\left(\mathbf{A 1}{ }^{\prime}\right)$ Todos os pontos de equilíbrio em $\partial A\left(x^{s}\right)$ são hiperbólicos, exceto possivelmente o equilíbrio $p$, o qual é um ponto de equilíbrio sela-nó do tipo zero.

(A4) A variedade estável dos pontos de equilíbrio em $\partial A\left(x^{s}\right)$ e a variedade $W^{c^{+}}(p)$ satisfazem a condição de transversalidade.

A suposição $\left(A 1^{\prime}\right)$ é mais fraca do que a suposição $(A 1)$ da seção 4.1, já que esta permite a presença de um ponto de equilíbrio não hiperbólico $p$ na fronteira da região de estabilidade.

Sob as suposições $\left(A 1^{\prime}\right),(A 3)$ e $(A 4)$, o próximo teorema oferece condições necessárias e suficientes para garantir que um ponto de equilíbrio sela-nó do tipo zero esteja na fronteira da região de estabilidade em termos de sua variedade estável e central. Estas condições sugerem procedimentos computacionais para verificar se um ponto de equilíbrio sela-nó do tipo zero está na fronteira da região de estabilidade.

Teorema 5.5.2. (Caracterizações adicionais de um ponto de equilíbrio sela-nó do tipo zero na fronteira da região de estabilidade): Seja $A\left(x^{S}\right)$ a região de estabilidade de um ponto de equilíbrio assintoticamente estável $x^{s}$ de (2.1). Se as suposições $\left(A 1^{\prime}\right),(A 3) e$ (A4) são satisfeitas e p é um ponto de equilíbrio sela-nó do tipo zero de (2.1), então:

(i) $p \in \partial A\left(x^{s}\right)$ se, e somente se, $W^{c^{+}}(p) \cap A\left(x^{s}\right) \neq \emptyset$.

(ii) $p \in \partial A\left(x^{s}\right)$ se, e somente se, $W^{s}(p) \subset \partial A\left(x^{s}\right)$.

Demonstração. $(i)(\Longleftarrow)$ Suponha que $W^{c^{+}}(p) \cap A\left(x^{s}\right) \neq \emptyset$, então existe $q^{\prime} \in W^{c^{+}}(p) \cap$ $A\left(x^{s}\right)$. Como $q^{\prime} \in W^{c^{+}}(p)$ então $\varphi\left(t, q^{\prime}\right) \rightarrow p$ quando $t \rightarrow-\infty$. Mas por outro lado, $q^{\prime} \in A\left(x^{s}\right)$ que é um conjunto invariante, assim $\varphi(t, p) \in A\left(x^{s}\right)$ para todo $t \in \mathbb{R}$ e consequentemente $p \in \overline{A\left(x^{s}\right)}$. Como $p \notin A\left(x^{s}\right)$ podemos afirmar que $p \in \partial A\left(x^{s}\right)$.

$(i)(\Longrightarrow)$ Suponha que $p \in \partial A\left(x^{s}\right)$. Do Teorema 5.5.1, podemos afirmar que $W_{l o c}^{c^{+}}(p) \cap$ $\overline{A\left(x^{s}\right)} \neq \emptyset$. Em particular, $W^{c^{+}}(p) \cap \overline{A\left(x^{s}\right)} \neq \emptyset$ já que $W_{l o c}^{c^{+}}(p) \subset W^{c^{+}}(p)$. Iremos mostrar, sob as suposições $\left(A 1^{\prime}\right),(A 3)$ e $(A 4)$ que $W^{c^{+}}(p) \cap \overline{A\left(x^{s}\right)} \neq \emptyset$ implica $W^{c^{+}}(p) \cap A\left(x^{s}\right) \neq \emptyset$. Seja $q^{*} \in W^{c^{+}}(p) \cap \overline{A\left(x^{s}\right)}$. Se $q^{*} \in A\left(x^{s}\right)$, então não existe nada a ser provado. Suponha que $q^{*} \in \partial A\left(x^{s}\right)$. A suposição $(A 3)$ garante a existência de um ponto de equilíbrio $p^{*} \in \partial A\left(x^{s}\right)$ tal que $\varphi\left(t, q^{*}\right) \longrightarrow p^{*}$ quando $t \longrightarrow \infty$. Dos Lemas 5.4.4 e 5.4.5, podemos afirmar que $p^{*} \neq p$. Como consequência da suposição $\left(A 1^{\prime}\right), p^{*}$ é um ponto de equilíbrio hiperbólico. Desde que $q^{*} \in W^{c^{+}}(p) \cap W^{s}\left(p^{*}\right)$, a suposição $(A 4)$ e o Lema 5.4.2 garantem que $p^{*}$ é um ponto de equilíbrio hiperbólico do tipo zero. Mas este fato leva-nos a um absurdo, pois $p^{*} \in \partial A\left(x^{s}\right)$. Portanto, $W^{c^{+}}(p) \cap A\left(x^{s}\right) \neq \emptyset$.

(ii) $(\Longleftarrow)$ Suponha que $W^{s}(p) \subset \partial A\left(x^{s}\right)$. Desde que $p \in W^{s}(p)$, então $p \in \partial A\left(x^{s}\right)$.

$($ ii $)(\Longrightarrow)$ Suponha que $p \in \partial A\left(x^{s}\right)$. Do item (i), temos que $W^{c^{+}}(p) \cap A\left(x^{s}\right) \neq \emptyset$. Seja 
$b \in W^{c^{+}}(p) \cap A\left(x^{s}\right)$. Em particular, $b \in W^{c^{+}}(p)$, ou seja, existe $T^{*}<0$ tal que $w=$ $\varphi\left(T^{*}, b\right) \in W_{l o c}^{c^{+}}(p)$. Por outro lado, como $b \in A\left(x^{s}\right)$ e $A\left(x^{s}\right)$ é um conjunto invariante, temos também que $w \in A\left(x^{s}\right)$. Sendo assim, $w \in W^{c^{+}}(p) \cap A\left(x^{s}\right)$. Considere $B(w ; \varepsilon)$ uma bola aberta com raio $\varepsilon$ centrada em $w$. O raio $\varepsilon$ pode ser escolhido suficientemente pequeno tal que $B(w ; \varepsilon) \subset A\left(x^{s}\right)$ visto que $A\left(x^{s}\right)$ é um conjunto aberto. Seja $q^{*}$ um ponto arbitrário de $W^{s}(p)$. Em particular, temos que $q=\varphi\left(T, q^{*}\right) \in W_{l o c}^{s}(p)$ para algum $T>0$. Considere um disco $D$ transversal a $W_{l o c}^{s}(p)$ em $q$ de dimensão 1 . Como uma consequência do Lema 5.2.3, existe um elemento $z \in D$ e um tempo $t^{*}>0$ tal que $\varphi\left(t^{*}, z\right) \in B(w ; \varepsilon)$. Desde que $A\left(x^{s}\right)$ é invariante, temos que $z \in A\left(x^{s}\right)$. Como $\varepsilon$ e o disco $D$ podem ser escolhidos arbitrariamente pequenos, então existem pontos de $A\left(x^{s}\right)$ arbitrariamente próximos a $q$. Portanto, $q \in \overline{A\left(x^{s}\right)}$. Desde que $W_{l o c}^{s}(p)$ não contém pontos em $A\left(x^{s}\right), q \in \partial A\left(x^{s}\right)$. Como a fronteira $\partial A\left(x^{s}\right)$ é invariante podemos afirmar que $q^{*}=\varphi(-T, q) \in \partial A\left(x^{s}\right)$. Explorando o fato que $q^{*}$ foi tomado arbitrariamente em $W^{s}(p)$, concluimos que $W^{s}(p) \subset \partial A\left(x^{s}\right)$.

A Figura 5.7 ilustra o Teorema 5.5.2. A figura mostra um ponto de equilíbrio sela-nó do tipo zero $p$ na fronteira da região de estabilidade de um ponto de equilíbrio assintoticamente estável $x^{s}$. A componente instável $W^{c^{+}}(p)$ da variedade central $W^{c}(p)$ intercepta a região de estabilidade $A\left(x^{s}\right)$, enquanto a variedade estável $W^{S}(p)$ está contida na fronteira da região de estabilidade.

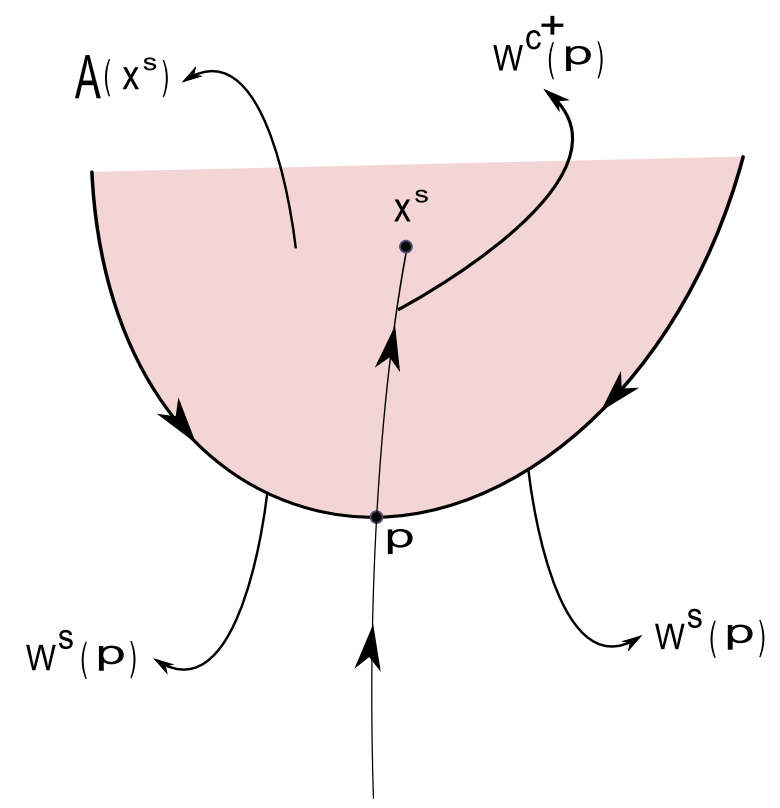

Figura 5.7: Um ponto de equilíbrio sela-nó do tipo zero $p$ na fronteira da região de estabilidade de um ponto de equilíbrio assintoticamente estável $x^{s}$. A componente instável $W^{c^{+}}(p)$ da variedade central $W^{c}(p)$ intercepta a região de estabilidade $A\left(x^{s}\right)$, ao passo que $W^{s}(p)$ está contida na fronteira da região de estabilidade.

Sob as suposições $\left(A 1^{\prime}\right),(A 2),(A 3)$ e $(A 4)$ e explorando os resultados do Teorema 4.1.1 e 5.5.2, obtemos o seguinte resultado: 
Teorema 5.5.3. (Pontos de equilíbrio hiperbólicos na fronteira da região de estabilidade): Seja $A\left(x^{S}\right)$ a região de estabilidade de um ponto de equilíbrio assintoticamente estável $x^{s}$ de (2.1). Se as suposições $\left(A 1^{\prime}\right),(A 2),(A 3)$ e (A4) são satisfeitas e $x^{*}$ é um ponto de equilíbrio hiperbólico de (2.1), então:

(i) $x^{*} \in \partial A\left(x^{s}\right)$ se, e somente se, $W^{u}\left(x^{*}\right) \cap A\left(x^{s}\right) \neq \emptyset$.

(ii) $x^{*} \in \partial A\left(x^{s}\right)$ se, e somente se, $W^{s}\left(x^{*}\right) \subseteq \partial A\left(x^{s}\right)$.

Demonstração. $(i)(\Leftarrow)$ Suponha que $W^{u}\left(x^{*}\right) \cap A\left(x^{s}\right) \neq \emptyset$, então existe $q^{\prime} \in W^{u}\left(x^{*}\right) \cap$ $A\left(x^{s}\right)$. Como $q^{\prime} \in W^{u}\left(x^{*}\right)$ então $\varphi\left(t, q^{\prime}\right) \rightarrow x^{*}$ quando $t \rightarrow-\infty$. Mas por outro lado, $q^{\prime} \in A\left(x^{s}\right)$ que é um conjunto invariante, assim $\varphi\left(t, q^{\prime}\right) \in A\left(x^{s}\right)$ para todo $t \in \mathbb{R}$ e consequentemente $x^{*} \in \overline{A\left(x^{s}\right)}$. Como $x^{*} \notin A\left(x^{s}\right)$ podemos afirmar que $x^{*} \in \partial A\left(x^{s}\right)$.

$(i)(\Rightarrow)$ Suponha que $x^{*} \in \partial A\left(x^{s}\right)$. Do Teorema 4.1.1, podemos afirmar que $\left(W^{u}\left(x^{*}\right)-\right.$ $\left.x^{*}\right) \cap \overline{A\left(x^{s}\right)} \neq \emptyset$. Iremos mostrar, sob as suposições $\left(A 1^{\prime}\right),(A 2)-(A 4)$ que $\left(W^{u}\left(x^{*}\right)-x^{*}\right) \cap$ $\overline{A\left(x^{s}\right)} \neq \emptyset$ implica $W^{u}\left(x^{*}\right) \cap A\left(x^{s}\right) \neq \emptyset$. Seja $q^{*} \in\left(W^{u}\left(x^{*}\right)-x^{*}\right) \cap \overline{A\left(x^{s}\right)}$. Se $q^{*} \in A\left(x^{s}\right)$, então não existe nada a ser provado. Suponha que $q^{*} \in \partial A\left(x^{s}\right)$. As suposições $\left(A 1^{\prime}\right) \mathrm{e}$ (A3) garantem que $q^{*} \in W^{s}(z)$ para algum ponto de equilíbrio $z \in \partial A\left(x^{s}\right)$, onde $z$ é um ponto de equilíbrio hiperbólico ou $z=p$ um ponto de equilíbrio sela-nó do tipo zero, ou seja, $q^{*} \in W^{u}\left(x^{*}\right) \cap W^{s}(z)$. Estudaremos separadamente as duas possibilidades para $z$ :

(1) Se $z=p$ é um ponto de equilíbrio sela-nó do tipo zero. Da suposição $(A 2)$, temse que $W^{u}\left(x^{*}\right)$ e $W^{s}(p)$ se interceptam transversalmente, então pelo Lema 5.4 .3 o ponto de equilíbrio $x^{*}$ é do tipo $k \geqslant 2$. Usando o Teorema 5.5.2 podemos afirmar que existe $q \in W^{c^{+}}(p) \cap A\left(x^{s}\right)$ já que $p \in \partial A\left(x^{s}\right)$. Como $A\left(x^{s}\right)$ é um conjunto aberto, existe $\varepsilon>0$ tal que $B(q ; \varepsilon) \subset A\left(x^{s}\right)$. Considere um disco $D$, contido em $W^{u}\left(x^{*}\right)$, de dimensão 1 e transversal a $W^{s}(p)$ em $q^{*}$. Como consequência do Lema 5.2.3, existem $T>0$ e $d \in D$ tal que $\varphi(T, d) \in B(q ; \varepsilon)$. A invariância de $A\left(x^{s}\right)$ garante que $d \in A\left(x^{s}\right), \operatorname{logo}, W^{u}\left(x^{*}\right) \cap A\left(x^{s}\right) \neq \emptyset$. (2) Se $z$ é um ponto de equilíbrio hiperbólico, a prova será feita por indução finita sobre a dimensão de $W^{u}\left(x^{*}\right)$

$-\operatorname{dim} W^{u}\left(x^{*}\right)=1$.

A suposição $(A 2)$ garante que $W^{u}\left(x^{*}\right)$ e $W^{s}(z)$ se interceptam transversalmente, então, pelo Lema 5.4.1, podemos afirmar que $z$ é um ponto de equilíbrio do tipo zero, levandonos a um absurdo, já que $z \in \partial A\left(x^{s}\right)$. Portanto, $W^{u}\left(x^{*}\right) \cap A\left(x^{s}\right) \neq \emptyset$ para todo ponto de equilíbrio $x^{*}$ do tipo 1 na fronteira $\partial A\left(x^{s}\right)$.

- Suponhamos que $W^{u}\left(x^{*}\right) \cap A\left(x^{s}\right) \neq \emptyset$ para todo ponto de equilíbrio $x^{*}$ na fronteira $\partial A\left(x^{s}\right)$ tal que $\operatorname{dim} W^{u}\left(x^{*}\right) \leqslant k$.

- Suponhamos agora que $\operatorname{dim} W^{u}\left(x^{*}\right)=k+1$.

A suposição $(A 2)$ garante que $W^{u}\left(x^{*}\right)$ e $W^{s}(z)$ se interceptam transversalmente, então, pelo Lema 5.4.1, podemos afirmar que $\operatorname{dim} W^{u}(z) \leqslant k$. Portanto, pela hipótese de indução $W^{u}(z) \cap A\left(x^{s}\right) \neq \emptyset$. Seja $y \in W^{u}(z) \cap A\left(x^{s}\right)$ e seja $B(y ; \varepsilon)$ uma bola aberta de raio $\varepsilon$, centrada em $y$. Como a região de estabilidade $A\left(x^{s}\right)$ é um conjunto aberto, $B(y ; \varepsilon) \subset A\left(x^{s}\right)$ para $\varepsilon$ suficientemente pequeno. Seja $N$ uma vizinhança de $q^{*}$ em $W^{u}\left(x^{*}\right)$. Esta vizi- 
nhança está imersa em uma variedade de dimensão $k+1$, além disso $N$ contém um disco $D$ de dimensão $k$ transversal a $W^{s}(z)$ no ponto $q^{*}$. Uma aplicação direta do Lema 5.2.1 permite-nos afirmar que existe um ponto $w \in D$ e um tempo $t^{*}>0$ tal que $\varphi\left(t^{*}, w \in\right.$ $B(y ; \varepsilon)$. A invariância de $A\left(x^{s}\right)$ garante que $w \in A\left(x^{s}\right)$, logo, $W^{u}\left(x^{*}\right) \cap A\left(x^{s}\right) \neq \emptyset$.

A prova do item (ii) é inteiramente análoga a prova do item $(i i)$ do Teorema 5.5.2 e por isso será omitida.

Observação 5.5.1. $O$ Teorema 5.5.3 é um resultado mais geral do que o Teorema 4.1.2, pois a condição de hiperbolicidade $(A 1)$, usada na prova do Teorema 4.1.2, é relaxada. Ainda assim, ele fornece as mesmas condições necessárias e suficientes do Teorema 4.1.2 para verificar se um ponto de equilíbrio hiperbólico está na fronteira da região de estabilidade em termos de suas variedades estáveis e instáveis.

Usando os resultados do Teorema 5.5.2 e Teorema 5.5.3, o próximo teorema fornece uma caracterização completa da fronteira da região de estabilidade quando existe um ponto de equilíbrio sela-nó do tipo zero em $\partial A\left(x^{s}\right)$.

Teorema 5.5.4. (Caracterização da fronteira da região de estabilidade): Seja $A\left(x^{s}\right)$ a região de estabilidade de um ponto de equilíbrio assintoticamente estável $x^{s}$ de (2.1). Se as suposições (A1'), (A2), (A3) e (A4) são satisfeitas então:

$$
\partial A\left(x^{s}\right)=\bigcup_{i} W^{s}\left(x^{i}\right) \bigcup W^{s}(p)
$$

onde $x^{i}, i=1,2, \ldots$ são os pontos de equilíbrios hiperbólicos em $\partial A\left(x^{s}\right)$ e p é um ponto de equilíbrio sela-nó do tipo zero que também pertence à fronteira $\partial A\left(x^{s}\right)$.

Demonstração. Se o ponto de equilíbrio hiperbólico $x^{i} \in \partial A\left(x^{s}\right)$, então, do Teorema 5.5.3, temos que $W^{s}\left(x^{i}\right) \subset \partial A\left(x^{s}\right)$. Desde que $p \in \partial A\left(x^{s}\right)$, temos que $W^{s}(p) \subset \partial A\left(x^{s}\right)$ pelo Teorema 5.5.2. Portanto, $\cup_{i} W^{s}\left(x^{i}\right) \cup W^{s}(p) \subset \partial A\left(x^{s}\right)$. Por outro lado, da suposição $(A 3)$, se $q \in \partial A\left(x^{s}\right)$, então $\varphi(t, q) \longrightarrow x^{i}$ para algum $i$ ou $\varphi(t, q) \longrightarrow p$ quando $t \longrightarrow \infty$. Como consequência do Lema 5.4.5, o ponto $q \notin W^{c^{-}}(p)$. Logo, podemos afirmar que $q \in W^{s}\left(x^{i}\right)$ ou $p \in W^{s}(p)$. Portanto $\partial A\left(x^{s}\right) \subseteq \cup_{i} W^{s}\left(x^{i}\right) \cup W^{s}(p)$ e o teorema está provado.

As demonstrações dos Teoremas 5.5.1, 5.5.2, 5.5.3 e 5.5.4 também podem ser encontradas em (AMARAL; ALBERTO, 2010a).

O Teorema 5.5.4 é uma generalização do Teorema 4.1.3. Ele mostra que a fronteira da região de estabilidade é composta pela união das variedades estáveis de todos os pontos de equilíbrio na fronteira da região de estabilidade, incluindo a variedade estável do ponto de equilíbrio sela-nó do tipo zero.

A Figura 5.8 ilustra a importância da suposição $(A 4)$ na demonstração do Teorema 5.5.2. Ela mostra um exemplo de um sistema dinâmico onde a componente instável 
$W^{c^{+}}(p)$ da variedade central de um ponto de equilíbrio sela-nó do tipo zero $p$ e a sua variedade estável $W^{s}(p)$ não satisfazem a condição de transversalidade na fronteira da região de estabilidade de um ponto de equilíbrio assintoticamente estável $x^{s}$. Observe, para este exemplo, que a variedade $W^{c^{+}}(p)$ não intercepta a região de estabilidade de $x^{s} \mathrm{e}$ a fronteira da região de estabilidade de $x^{s}$ não é composta pela variedade estável do ponto de equilíbrio sela-nó do tipo zero $p$ que pertence à fronteira. Os Teoremas 5.5.2 e 5.5.4 não podem ser aplicados para este exemplo visto que a condição $(A 4)$ é violada.

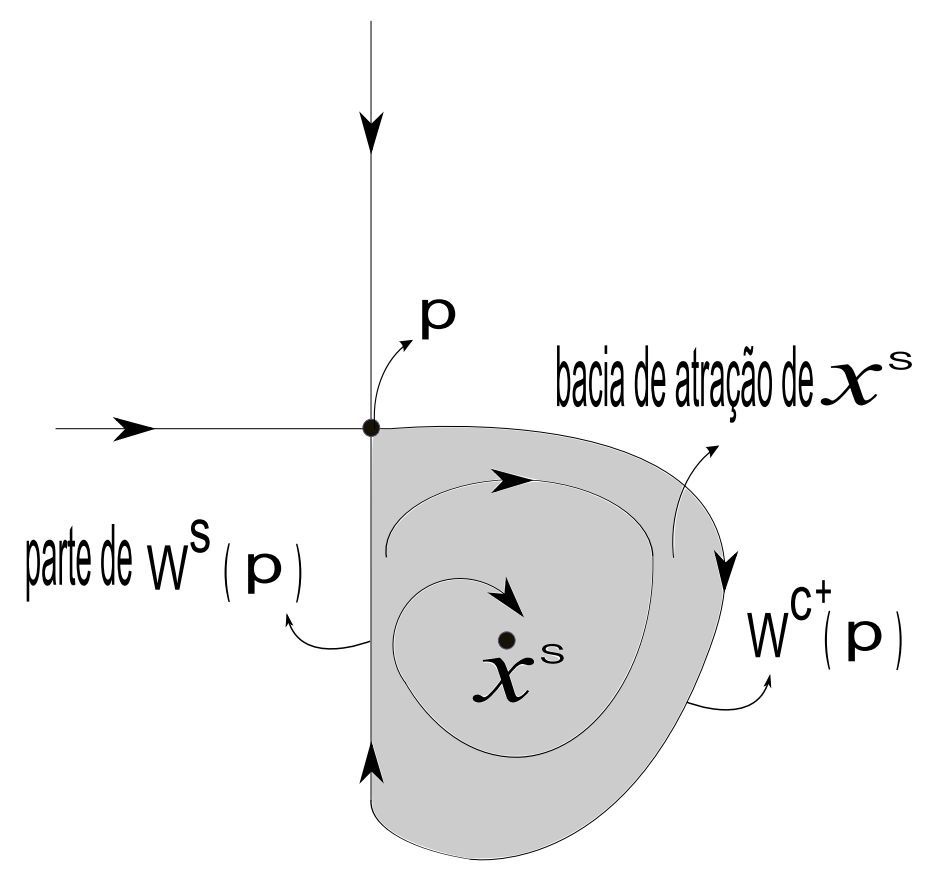

Figura 5.8: Exemplo de um sistema dinâmico onde a variedade $W^{c^{+}}(p)$ e a variedade estável $W^{s}(p)$ de um ponto de equilíbrio sela-nó do tipo zero $p$ não satisfazem a condição de transversalidade na fronteira da região de estabilidade de um ponto de equilíbrio assintoticamente estável $x^{s}$.

Exploraremos a seguir dois exemplos que ilustram os Teoremas 5.5.2, 5.5.3 e 5.5.4.

Exemplo 5.5.1. Considere o mesmo sistema (5.1) dado no ínicio da seção anterior.

Vimos que, o sistema (5.1) possui, três pontos de equilíbrio; são eles $p=(0 ;-1)$, um ponto de equilíbrio sela-nó do tipo zero, $x^{s}=(-1 ; 0)$, um ponto de equilíbrio hiperbólico assintoticamente estável e $x^{*}=(1 ; 0)$, um ponto de equilíbrio hiperbólico do tipo um. Ambos, o ponto de equilíbrio sela-nó do tipo zero $p=(0 ;-1)$ e o ponto de equilíbrio do tipo um $x^{*}=(1 ; 0)$ pertencem à fronteira da região de estabilidade de $x^{s}=(-1 ; 0)$, ver Figura 5.6. As suposições $\left(A 1^{\prime}\right),(A 2)-(A 4)$ são satisfeitas. A componente instável $W^{c^{+}}(0 ;-1)$ da variedade central do ponto de equilíbrio sela-nó do tipo zero $p=(0 ;-1)$ intercepta a região de estabilidade de $x^{s}=(-1 ; 0)$, e a variedade estável $W^{s}(0 ;-1)$ está contida na fronteira da região de estabilidade de $x^{s}=(-1 ; 0)$, de acordo com o Teorema 5.5.2, ver Figura 5.6. A variedade instável $W^{u}(1 ; 0)$ do ponto de equilíbrio hiperbólico 
do tipo um $x^{*}=(1 ; 0)$ intercepta a região de estabilidade de $x^{s}=(-1 ; 0)$ e a variedade estável $W^{s}(1 ; 0)$ está contida na fronteira da região de estabilidade de $x^{s}=(-1 ; 0)$, de acordo com o Teorema 5.5.3, ver Figura 5.6. A fronteira da região de estabilidade de $x^{s}=(-1 ; 0)$ é formada, de acordo com o Teorema 5.5.4, pela união da variedade estável do ponto de equilíbrio hiperbólico do tipo um $x^{*}=(1 ; 0)$, a curva azul da Figura 5.6, com a variedade estável do ponto de equilíbrio sela-nó do tipo zero $p=(0 ;-1)$, a curva vermelha da Figura 5.6.

Exemplo 5.5.2. Considere o sistema de equações diferenciais

$$
\begin{aligned}
& \dot{x}=-x^{3}+x+\frac{2}{3 \sqrt{3}} \\
& \dot{y}=-y
\end{aligned}
$$

onde $(x ; y) \in \mathbb{R}^{2}$.

O sistema (5.2) possui, dois pontos de equilíbrio; são eles $p=(-0,5774 ; 0)$, um ponto de equilíbrio sela-nó do tipo zero e $x^{s}=(1,1547 ; 0)$, um ponto de equilíbrio hiperbólico assintoticamente estável. O ponto de equilíbrio sela-nó do tipo zero $p=(-0,5774 ; 0)$ pertence à fronteira da região de estabilidade de $x^{s}=(1,1547 ; 0)$, ver Figura 5.9. As suposições $\left(A 1^{\prime}\right),(A 2)-(A 4)$ são satisfeitas. A componente instável $W^{c^{+}}(-0,5774 ; 0)$ da variedade central do ponto de equilíbrio sela-nó do tipo zero $p=(-0,5774 ; 0)$ intercepta a região de estabilidade de $x^{s}=(1,1547 ; 0)$, e a variedade estável $W^{s}(-0,5774 ; 0)$ está contida na fronteira da região de estabilidade de $x^{s}=(1,1547 ; 0)$, de acordo com o Teorema 5.5.2, ver Figura 5.9. A fronteira da região de estabilidade de $x^{s}=(1,1547 ; 0)$ é formada, de acordo com o Teorema 5.5.4, pela variedade estável do ponto de equilíbrio sela-nó do tipo zero $p=(-0,5774 ; 0)$, a curva azul da Figura 5.9.

\subsection{Região de estabilidade fraca}

No Capítulo 8, investigaremos o comportamento da fronteira da região de estabilidade na vizinhança de um parâmetro de bifurcação sela-nó do tipo zero. Mas primeiramente precisamos explorar as propriedades de um conjunto importante neste estudo. Este conjunto será definido a seguir e denominado de região de estabilidade fraca.

Definição 5.6.1. (Região de Estabilidade Fraca) Seja p um ponto de equilíbrio sela-nó do tipo zero de (2.1). A região de estabilidade fraca de $p$ é o conjunto $S(p)$ dos pontos $q \in\left(\mathbb{R}^{n}-W^{S}(p)\right)$ cujas trajetórias convergem para $p$ quando $t \longrightarrow+\infty$ :

$$
S(p)=\left\{q \in\left(\mathbb{R}^{n}-W_{\lambda_{0}}^{s}(p)\right): \varphi(t, q) \longrightarrow p \text { quando } t \longrightarrow+\infty\right\}
$$

Para qualquer vizinhança $U$ de $p$, o conjunto $U^{-} \subset S(p)$. 


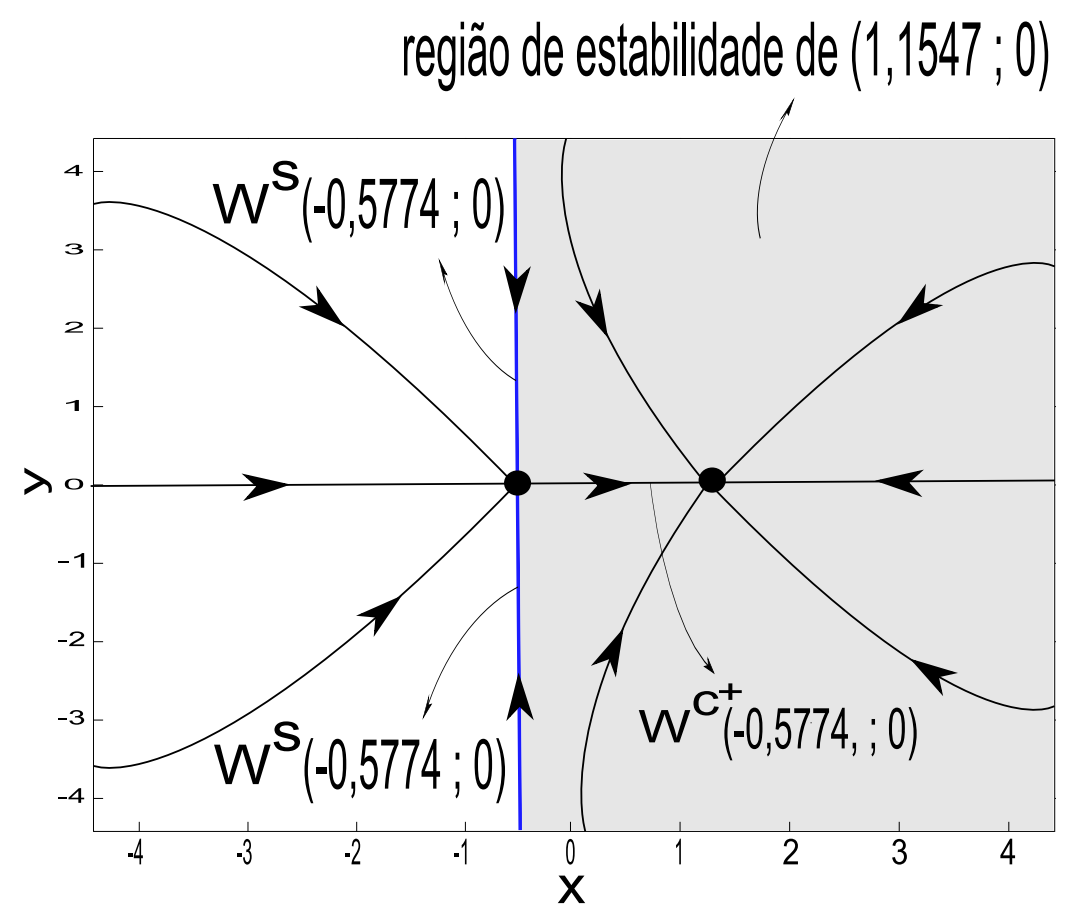

Figura 5.9: O retrato de fase do sistema (5.2). A fronteira da região de estabilidade do ponto de equilíbrio assintoticamente estável $(1,1547 ; 0)$ é formada pela variedade estável do ponto de equilíbrio sela-nó do tipo zero $(-0,5774 ; 0)$, a curva em azul.

O teorema a seguir permite concluir que a região de estabilidade fraca é um conjunto aberto.

Teorema 5.6.1. Seja p um ponto de equilíbrio sela-nó do tipo zero de (2.1). Então a região de estabilidade fraca $S(p)$ é um conjunto aberto em $\mathbb{R}^{n}$.

Demonstração. Seja $q \in S(p)$, então $q \notin W^{S}(p)$ e $\varphi(t, q) \longrightarrow p$ quando $t \longrightarrow+\infty$, em particular, existe $T>0$ tal que $\varphi(T, q) \in U^{-}$. Como $U^{-}$é um conjunto aberto existe $\varepsilon>0$ tal que a bola aberta $B(\varphi(T, q) ; \varepsilon)$ está contida em $U^{-}$, isto é, $B(\varphi(T, q) ; \varepsilon) \subset$ $U^{-}$. Da continuidade das soluções com relação às condições iniciais, existe $\delta>0$ tal que $\varphi\left(T, q^{*}\right) \in B(\varphi(T, q) ; \varepsilon)$ sempre que $q^{*} \in B(q ; \delta)$. Utilizando a propriedade que $\varphi\left(t_{1}+t_{2}, x\right)=\varphi\left(t_{1}, \varphi\left(t_{2}, x\right)\right)$ para todo $x \in \mathbb{R}^{n}$ e $t_{1}, t_{2} \in \mathbb{R}$, temos que $\varphi\left(t, q^{*}\right)=\varphi(t-$ $\left.T, \varphi\left(T, q^{*}\right)\right)=\varphi\left(s, \varphi\left(T, q^{*}\right)\right)$ onde $s=t-T$. Portanto,

$$
\lim _{t \longrightarrow \infty} \varphi\left(t, q^{*}\right)=\lim _{s \longrightarrow \infty} \varphi\left(s, \varphi\left(T, q^{*}\right)\right)=p
$$

Consequentemente, existe $\delta>0$ tal que todo ponto $q^{*}$ pertencendo a bola aberta $B(q ; \delta)$ também pertence a região de estabilidade fraca $S(p)$, isto é, $B(q ; \delta) \subset S(p)$. Portanto, $S(p)$ é um conjunto aberto.

Mostraremos no próximo teorema que a região de estabilidade fraca é um conjunto invariante. 
Teorema 5.6.2. Seja p um ponto de equilíbrio sela-nó do tipo zero de (2.1). Então a região de estabilidade fraca $S(p)$ é um conjunto invariante.

Demonstração. Seja $q \in S(p)$, então $q \notin W^{s}(p)$ e $\varphi(t, q) \longrightarrow p$ quando $t \longrightarrow+\infty$. Seja $q^{*}=\varphi(s, q)$ para algum $s \in \mathbb{R}$ arbitrário. Então, $q^{*} \notin W^{s}(p)$ e utilizando a propriedade que $\varphi\left(t_{1}+t_{2}, x\right)=\varphi\left(t_{1}, \varphi\left(t_{2}, x\right)\right)$ para todo $x \in \mathbb{R}^{n}$ e $t_{1}, t_{2} \in \mathbb{R}$, temos que $\varphi\left(t, q^{*}\right)=$ $\varphi(t, \varphi(s, q))=\varphi(t+s, q)$. Para cada $s$ fixo temos que $t+s \longrightarrow+\infty$ quando $t \longrightarrow+\infty$ e consequentemente

$$
\varphi\left(t, q^{*}\right)=\varphi(t+s, q) \longrightarrow p \quad \text { quando } t \longrightarrow+\infty
$$

Portanto, $q^{*} \in S(p)$ e da arbitrariedade da escolha de $q$ podemos afirmar que $S(p)$ é um conjunto invariante com relação ao sistema (2.1).

Observação 5.6.1. Se $x^{s}$ é um ponto de equilíbrio hiperbólico assintoticamente estável de (2.1), e $p$ é um ponto de equilíbrio sela-nó do tipo zero pertencente à fronteira $\partial A\left(x^{s}\right)$ então $\partial S(p) \cap \partial A\left(x^{s}\right) \neq \emptyset$. Como $p \in \partial A\left(x^{s}\right)$, qualquer vizinhança $U$ de $p$ intercepta $A\left(x^{S}\right)$, isto é, intercepta o complementar de $S(p)$ já que $S(p) \cap A\left(x^{s}\right)=\emptyset$. Por outro lado $U$ intercepta $S(p)$, já que $U^{-} \subset S(p)$. Logo, $p \in \partial S(p)$ e consequentemente $\partial S(p) \cap$ $\partial A\left(x^{s}\right) \neq \emptyset$.

Nas condições da observação anterior, denotaremos o conjunto $\partial S^{*}(p)$ como sendo o conjunto dos pontos que pertencem a fronteira $\partial S(p)$ e não pertencem a fronteira $\partial A\left(x^{S}\right)$, ou seja, $\partial S^{*}(p)=\partial S(p)-\partial A\left(x^{s}\right)$.

O teorema a seguir oferece uma condição necessária e suficiente para garantir que um ponto de equilíbrio hiperbólico esteja no conjunto $\partial S(p)$.

\section{Teorema 5.6.3. (Ponto de equilíbrio hiperbólico na fronteira da região de estabilidade}

fraca): Sejam p um ponto de equilíbrio sela-nó do tipo zero e $x^{*}$ um ponto de equilíbrio hiperbólico de (2.1). Então:

$$
x^{*} \in \partial S(p) \Leftrightarrow\left(W_{l o c}^{u}\left(x^{*}\right)-x^{*}\right) \cap \overline{S(p)} \neq \emptyset
$$

Demonstração. $(\Longleftarrow)$ Suponha que $\left(W_{l o c}^{u}\left(x^{*}\right)-x^{*}\right) \cap \overline{S(p)} \neq \emptyset$. Então existe $x \in W_{l o c}^{u}\left(x^{*}\right) \cap$ $\overline{S(p)} \operatorname{com} x \neq x^{*}$. Como $x \in W_{l o c}^{u}\left(x^{*}\right)$, então $\varphi(t, x) \longrightarrow x^{*}$ quando $t \longrightarrow-\infty$. Por outro lado, $x \in \overline{S(p)}$ e o conjunto $\overline{S(p)}$ é invariante, assim $\varphi(t, x) \in \overline{S(p)}$ para todo $t \leqslant 0$. Como consequência, $x^{*} \in \overline{S(p)}$. Desde que $x^{*} \notin S(p)$, temos que $x^{*} \in\left(\mathbb{R}^{n}-S(p)\right)$. Portanto, $x^{*} \in \partial S(p)$.

$(\Longrightarrow)$ Suponha que $x^{*} \in \partial S(p)$. Sejam $D$ um domínio fundamental de $W_{l o c}^{u}\left(x^{*}\right)$ e $D_{\varepsilon}:=$ $\left\{x \in \mathbb{R}^{n}: d(x, D)<\varepsilon\right\}$ uma vizinhança fundamental de $D$ para algum $\varepsilon>0$. Como consequência do Lema 5.2.1, existe uma vizinhança $U$ de $x^{*}$ tal que $\cup_{t \leqslant 0} \varphi_{\lambda_{0}}\left(t, D_{\varepsilon}\right)$ contém o conjunto $U-W_{l o c}^{s}\left(x^{*}\right)$. Como $x^{*} \in \partial S(p)$, então $U \cap S(p) \neq \emptyset$. Por outro lado, 
$W_{\text {loc }}^{s}\left(x^{*}\right) \cap S(p)=\emptyset$. Logo, $\left(U-W_{\text {loc }}^{s}\left(x^{*}\right)\right) \cap S(p) \neq \emptyset$. Isto significa que existe algum ponto $p^{*} \in D_{\varepsilon}$ e um tempo $t^{*}$ tal que $\varphi_{\lambda_{0}}\left(t^{*}, p^{*}\right) \in S(p)$. A invariância de $S(p)$ garante que $p^{*} \in S(p)$. Como $\varepsilon$ pode ser esolhido arbitrariamente pequeno, podemos encontrar uma sequência de pontos $\left\{p_{i}^{*}\right\} \operatorname{com} p_{i}^{*} \in S(p)$ para todo $i=1,2, \ldots$ e $d\left(p_{i}^{*}, D\right) \rightarrow 0$ quando $i \rightarrow+\infty$. Por construção, esta sequência é limitada, portanto possui subsequência convergente, isto é, $p_{i_{j}}^{*} \rightarrow q$ quando $i_{j} \rightarrow+\infty$. Assim, $d\left(p_{i_{j}}^{*}, D\right) \rightarrow d(q, D)$ quando $i_{j} \rightarrow+\infty$, e consequentemente $d(q, D)=0$, ou seja, $q \in \bar{D} \subset\left(W_{l o c}^{u}\left(x^{*}\right)-x^{*}\right)$. Portanto, $q \in\left(W_{l o c}^{u}\left(x^{*}\right)-x^{*}\right) \cap \overline{S(p)}$ como queríamos demonstrar.

Com suposições adicionais, uma versão mais forte do Teorema 5.6.3 pode ser obtida:

Teorema 5.6.4. Sejam $x^{s}$ um ponto de equilíbrio assintoticamente estável de (2.1), e p um ponto de equilíbrio sela-nó do tipo zero pertencente à fronteira $\partial A\left(x^{s}\right)$. Se as suposições $\left(A 1^{\prime}\right),(A 2),(A 3)$ e (A4) são satisfeitas em $\partial A\left(x^{s}\right)$ e em $\partial S(p)$, e $x^{*}$ é um ponto de equilíbrio hiperbólico de (2.1), então:

(i) $S$ e $x^{*} \in \partial S^{*}(p)$, então $W^{u}\left(x^{*}\right) \cap S(p) \neq \emptyset$.

(ii) Se $x^{*} \in \partial S^{*}(p)$, então $W^{S}\left(x^{*}\right) \subset \partial S(p)$.

Demonstração. (i) Se $x^{*} \in \partial S^{*}(p)$, então pelo Teorema 5.6.3 temos que $\left(W_{l o c}^{u}\left(x^{*}\right)-x^{*}\right) \cap$ $\overline{S(p)} \neq \emptyset$. Vamos mostrar sob as hipótese $\left(A 1^{\prime}\right),(A 2)-(A 4)$, que $\left(W_{l o c}^{u}\left(x^{*}\right)-x^{*}\right) \cap \overline{S(p)} \neq$ $\emptyset$ implica em $W_{l o c}^{u}\left(x^{*}\right) \cap S(p) \neq \emptyset$. Seja $p^{*} \in\left(W_{l o c}^{u}\left(x^{*}\right)-x^{*}\right) \cap \overline{S(p)}$. Se $p^{*} \in S(p)$ não existe nada a ser provado. Suponha que $p^{*} \in \partial S(p)$. As suposições $\left(A 1^{\prime}\right)$ e $(A 3)$ garantem que $p^{*} \in W^{S}(z)$ para algum ponto de equilíbrio $z$, onde $z$ é um ponto de equilíbrio hiperbólico em $\partial S(p)$ ou um ponto de equilíbrio sela-nó do tipo zero. Eliminaremos a hipótese de $z$ ser o ponto de equilíbrio sela-nó do tipo zero. Se $z$ for o ponto de equilíbrio sela-nó do tipo zero, sabendo que $z$ pertence à fronteira $\partial A\left(x^{s}\right)$ e as suposições $\left(A 1^{\prime}\right),(A 3)$ e (A4) são satisfeitas, o Teorema 5.5.2 garante que $W^{s}(z) \subset \partial A\left(x^{s}\right)$, ou seja, $p^{*} \in \partial A\left(x^{s}\right)$ e consequentemente $W^{u}\left(x^{*}\right) \cap \partial A\left(x^{s}\right) \neq \emptyset$, isto é, $x^{*} \in \partial A\left(x^{s}\right)$, o que é um absurdo já que $x^{*} \in \partial S^{*}(p)=\partial S(p)-\partial A\left(x^{s}\right)$. Portanto, $p^{*} \in W^{u}\left(x^{*}\right) \cap W^{s}(z)$ e $z$ é necessariamente um ponto de equilíbrio hiperbólico. O restante da prova será feita por indução finita sobre a dimensão de $W^{u}\left(x^{*}\right)$

(1) $\operatorname{dim} W^{u}\left(x^{*}\right)=1$.

A suposição $(A 2)$ garante que $W^{u}\left(x^{*}\right)$ e $W_{\lambda_{0}}^{s}(z)$ se interceptam transversalmente, então pelo Lema 5.4.1 podemos afirmar que $z$ é um ponto de equilíbrio hiperbólico do tipo zero, levando-nos a um absurdo, já que $z \in \partial S(p)$. Portanto, $W^{u}\left(x^{*}\right) \cap S(p) \neq \emptyset$ para todo ponto de equilíbrio $x^{*}$ do tipo 1 no conjunto $\partial S^{*}(p)$.

(2) Suponhamos que $W^{u}\left(x^{*}\right) \cap S(p) \neq \emptyset$ para todo ponto de equilíbrio $x^{*}$ no conjunto $\partial S^{*}(p)$ tal que $\operatorname{dim} W^{u}\left(x^{*}\right) \leqslant k$.

(3) Suponhamos agora que $\operatorname{dim} W^{u}\left(x^{*}\right)=k+1$.

Se $z$ for hiperbólico, a suposição $(A 2)$ garante que $W^{u}\left(x^{*}\right)$ e $W_{\lambda_{0}}^{s}(z)$ se interceptam transversalmente, então pelo Lema 5.4.1 podemos afirmar que $\operatorname{dim} W^{u}(z) \leqslant k$. Portanto, pela 
hipótese de indução $W^{u}(z) \cap S(p) \neq \emptyset$. Seja $y \in W^{u}(z) \cap S(p)$ e seja $B(y ; \varepsilon)$ uma bola aberta de raio $\varepsilon$, centrada em $y$. Como a região de estabilidade fraca $S(p)$ é um conjunto aberto, $B(y ; \varepsilon) \subset S(p)$ para $\varepsilon$ suficientemente pequeno. Seja $N$ uma vizinhança de $p^{*}$ em $W^{u}\left(x^{*}\right)$. Esta vizinhança está imersa em uma variedade de dimensão $k+1$, além disso $N$ contém um disco $D$ de dimensão $k$ transversal a $W^{s}(z)$ no ponto $p^{*}$. Uma aplicação direta do Lema 5.2.1 permite-nos afirmar que existe um ponto $w \in D$ e um tempo $t^{*}>0$ tal que $\varphi\left(t^{*}, w\right) \in B(y ; \varepsilon)$. A invariância de $S(p)$ garante que $w \in S(p), \log 0, W^{u}\left(x^{*}\right) \cap S(p) \neq \emptyset$. A prova do item (ii) é inteiramente análoga a prova do item (ii) do Teorema 5.5.2 e por isso será omitida.

Exibiremos a seguir um exemplo para ilustrar o conceito de região de estabilidade fraca.

Exemplo 5.6.1. Considere o sistema de equações diferenciais

$$
\begin{aligned}
& \dot{x}=x^{4}-1,25 x^{2}-y+0,25 \\
& \dot{y}=-y+0,25
\end{aligned}
$$

onde $(x ; y) \in \mathbb{R}^{2}$.

O sistema (5.3) possui, três pontos de equilíbrio; são eles $p=(0 ; 0,25)$ um ponto de equilíbrio sela-nó do tipo zero, $x^{s}=(-1,11 ; 0,25)$ um ponto de equilíbrio assintoticamente estável e $x^{*}=(1,11 ; 0,25)$ um ponto de equilíbrio hiperbólico do tipo um. $\mathrm{O}$ ponto de equilíbrio sela-nó do tipo zero pertence à fronteira da região de estabilidade de $x^{s}=(-1,11 ; 0,25)$ e o ponto de equilíbrio hiperbólico do tipo um $x^{*}=(1,11 ; 0,25)$ pertence ao conjunto $\partial S^{*}(0 ; 0,25)$, ver Figura 5.10. A variedade instável $W^{u}(1,11 ; 0,25)$ de $x^{*}=(1,11 ; 0,25)$ intercepta a região de estabilidade fraca $S(0 ; 0,25)$ e a variedade estável $W^{s}(1,11 ; 0,25)$ de $x^{*}=(1,11 ; 0,25)$, a curva azul da Figura 5.10, está contida na fronteira da região de estabilidade fraca $\partial S(0 ; 0,25)$, de acordo com o Teorema 5.6.4. 


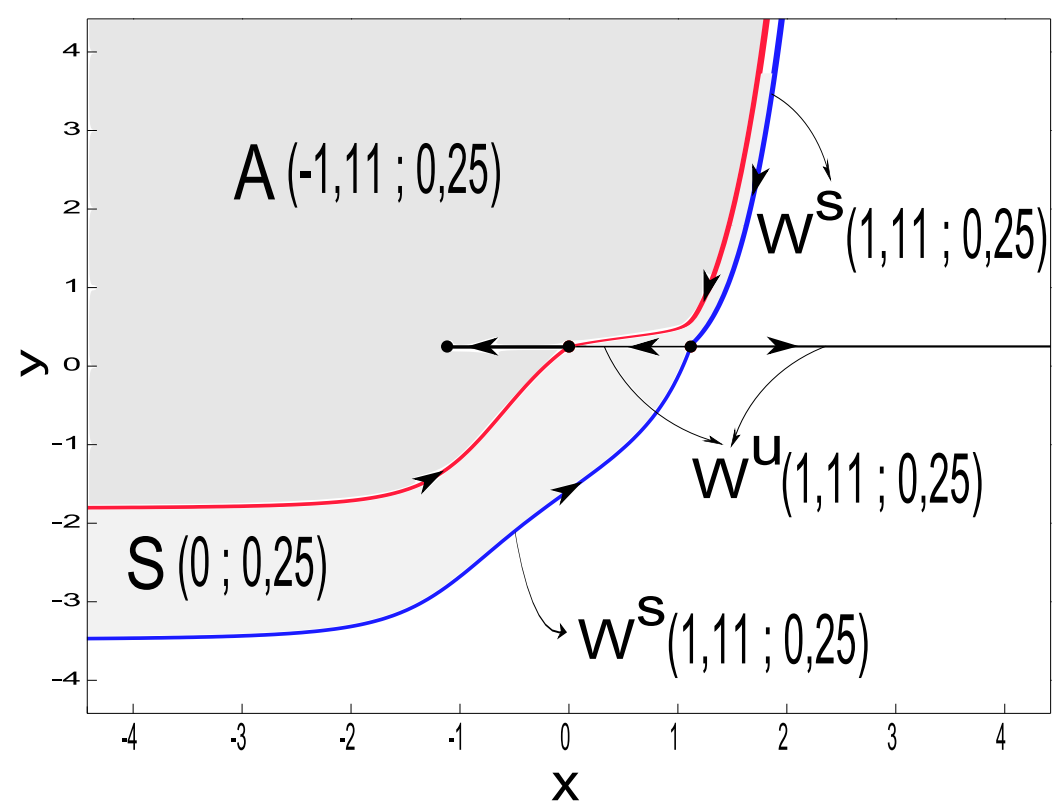

Figura 5.10: O retrato de fase do sistema (5.3). A fronteira da região de estabilidade fraca $\partial S(0 ; 0,25)$ é forma pela união da variedade estável do ponto de equilíbrio sela-nó do tipo zero $(0 ; 0,25)$, a curva em vermelho, com a variedade estável do ponto de equilíbrio $(1,11 ; 0,25)$, a curva em azul. 


\section{PERSISTÊNCIA DA REGIÃO DE ESTABILIDADE}

No capítulo anterior, exibimos uma caracterização da fronteira da região de estabilidade para sistemas dinâmicos autônomos não-lineares (2.1) na presença de um ponto de equilíbrio não hiperbólico sela-nó do tipo zero. A partir deste capítulo, estudaremos o comportamento do sistema dinâmico autônomo não-linear $\dot{x}=f(x, \lambda)$ sujeito a variação do parâmetro escalar $\lambda$. Em particular investigaremos o comportamento da região de estabilidade sob a influência de pequenas variações do parâmetro $\lambda$. Reservaremos para este capítulo discussões a respeito da persistência da região de estabilidade e da caracterização de sua fronteira sob pequenas variações do parâmetro. Na Seção 6.1, estudaremos a persistência da região de estabilidade e a persistência dos pontos de equilíbrio hiperbólicos na fronteira da região de estabilidade. Na seção 6.2, exibiremos exemplos que ilustram os resultados da seção anterior.

\subsection{Persistência dos pontos de equilíbrio hiperbólicos na fronteira da região de estabilidade}

Considere a família de equações diferenciais

$$
\dot{x}=f(x, \lambda)
$$

com $x \in \mathbb{R}^{n}, \lambda \in \mathbb{R}$ e $f: \mathbb{R}^{n} \times \mathbb{R} \longrightarrow \mathbb{R}^{n}$ um campo vetorial de classe $C^{r}$, com $r \geqslant 1$.

Dado $x_{\lambda_{*}}^{s}$ um ponto de equilíbrio hiperbólico assintoticamente estável de (6.1) para $\lambda=\lambda_{*}$, deseja-se saber se a região de estabilidade $A_{\lambda_{*}}\left(x_{\lambda_{*}}^{s}\right)$ persiste sob pequenas variações do parâmetro $\lambda$. O Teorema da Função Implícita é uma das ferramentas que permite estudar a persistência da região de estabilidade sob pequenas variações do parâmetro $\lambda$.

Teorema 6.1.1. (LIMA, 2006)(Teorema da Função Implícita) Sejam U um subconjunto aberto de $\mathbb{R}^{m} \times \mathbb{R}^{n}$ e $f: U \rightarrow \mathbb{R}^{n}$ uma função de classe $C^{k}, k \geqslant 1$. Considere um ponto $(\bar{x}, \bar{y}) \in U$, onde $\bar{x} \in \mathbb{R}^{m}$ e $\bar{y} \in \mathbb{R}^{n}$, com $f(\bar{x}, \bar{y})=c$. Se a matriz $n \times n D_{y} f(\bar{x}, \bar{y})$ das derivadas parciais de $f$ com relação a y é invertível, então existem conjuntos abertos $V_{m} \subset \mathbb{R}^{m}$ e $V_{n} \subset \mathbb{R}^{n} \operatorname{com}(\bar{x}, \bar{y}) \in V_{m} \times V_{n} \subset U$ e uma única função $\psi: V_{m} \rightarrow V_{n}$ de classe 
$C^{k}$ tal que $f(x, \psi(x))=$ c para todo $x \in V_{m}$. Além disso, $f(x, y) \neq c$ se $(x, y) \in V_{m} \times V_{n} e$ $y \neq \psi(x)$. A derivada da função $\psi$ é dada pela fórmula

$$
D \psi(x)=-\left[D_{y} f(x, \psi(x))\right]^{-1} D_{x} f(x, \psi(x)) .
$$

Se $x_{\lambda_{*}}$ é um ponto de equilíbrio hiperbólico do sistema (6.1) para $\lambda=\lambda_{*}$, então $D_{x} f_{\lambda_{*}}\left(x_{\lambda_{*}}\right)$ é invertível, sendo assim o Teorema da Função Implícita garante que em uma vizinhança de $x_{\lambda_{*}}$ continua existindo um único ponto de equilíbrio hiperbólico $x_{\lambda}$ do sistema perturbado (6.1) para todo $\lambda$ próximo a $\lambda_{*}$. Em outras palavras, um ponto de equilíbrio hiperbólico persiste sob pequenas variações do parâmetro $\lambda$. Além disso, usando a continuidade dos autovalores de $D_{x} f_{\lambda_{*}}\left(x_{\lambda_{*}}\right)$ com relação aos parâmetros (KATO, 1995), podemos afirmar também que o tipo de estabilidade do ponto de equilíbrio perturbado $x_{\lambda}$ é o mesmo do ponto de equilíbrio $x_{\lambda_{*}}$, ou seja, se $x_{\lambda_{*}}$ é um ponto de equilíbrio hiperbólico do tipo $k$, então o ponto de equilíbrio perturbado $x_{\lambda}$ é também um ponto de equilíbrio do tipo $k$.

Diante do que foi discutido anteriormente, para o caso particular de $x_{\lambda_{*}}^{s}$ ser um ponto de equilíbrio hiperbólico assintoticamente estável de (6.1) para $\lambda=\lambda_{*}$, podemos afirmar que existe $\delta>0$ tal que o sistema (6.1) possui um único ponto de equilíbrio hiperbólico assintoticamente estável $x_{\lambda}^{s}$ em uma vizinhança de $x_{\lambda_{*}}^{s}$ para todo $\lambda \in\left(\lambda_{*}-\delta, \lambda_{*}+\delta\right)$. Portanto, faz sentido estudar a região de estabilidade perturbada $A_{\lambda}\left(x_{\lambda}^{s}\right)$ e como esta se comporta sob a influência da variação do parâmetro $\lambda$.

Se $x_{\lambda_{*}}^{s}$ é um ponto de equilíbrio hiperbólico assintoticamente estável de (6.1), para $\lambda=$ $\lambda_{*}$ e se o sistema (6.1) satisfaz as suposições $(A 1)-(A 3)$ para $\lambda=\lambda_{*}$, então a fronteira da região de estabilidade $\partial A_{\lambda_{*}}\left(x_{\lambda_{*}}^{s}\right)$ é caracterizada, de acordo com o Teorema 4.1.3, como união das variedades estáveis dos pontos de equilíbrio instáveis que pertencem a fronteira da região de estabilidade. Mais precisamente, se $x_{\lambda_{*}}^{i}, i=1,2, \ldots$, são os pontos de equilíbrio em $\partial A_{\lambda_{*}}\left(x_{\lambda_{*}}^{s}\right)$ então

$$
\partial A_{\lambda_{*}}\left(x_{\lambda_{*}}^{s}\right)=\bigcup_{i} W_{\lambda_{*}}^{s}\left(x_{\lambda_{*}}^{i}\right)
$$

O teorema a seguir estuda a persistência dos pontos de equilíbrio hiperbólicos na fronteira da região de estabilidade perturbada para variações do parâmetro $\lambda$ próximo a $\lambda^{*}$

Teorema 6.1.2. (CHIANG; CHU, 1995)(Persistência dos pontos de equilíbrio hiperbólicos na fronteira da região de estabilidade) Seja $x_{\lambda^{*}}^{s}$ um ponto de equilíbrio hiperbólico assintoticamente estável de (6.1), para $\lambda=\lambda_{*} e A_{\lambda^{*}}\left(x_{\lambda^{*}}^{s}\right)$ sua região de estabilidade. Sejam $x_{\lambda^{*}}^{i}, i=1, \ldots, k$ os pontos de equilíbrio hiperbólicos em $\partial A_{\lambda^{*}}\left(x_{\lambda^{*}}^{s}\right)$ e admita que as suposições $(A 1)-(A 3)$ estejam satisfeitas para $\lambda=\lambda_{*}$. Então, existe um número positivo $\varepsilon$ tal que, os pontos de equilíbrio perturbados $x_{\lambda}^{i}, i=1, \ldots, k$ também pertencem à fron- 
teira da região de estabilidade perturbada de $\partial A_{\lambda}\left(x_{\lambda}^{s}\right)$ para todo $\lambda \in\left(\lambda^{*}-\varepsilon, \lambda^{*}+\varepsilon\right)$.

Como uma consequência direta dos Teoremas 4.1.2e 6.1.2 obtemos o seguinte corolário:

Corolário 6.1.1. Seja $x_{\lambda^{*}}^{s}$ um ponto de equilíbrio hiperbólico assintoticamente estável de (6.1), para $\lambda=\lambda_{*}$ e $A_{\lambda^{*}}\left(x_{\lambda^{*}}^{s}\right)$ sua região de estabilidade. Sejam $x_{\lambda^{*}}^{i}, i=1, \ldots, k$ os pontos de equilíbrio hiperbólicos em $\partial A_{\lambda^{*}}\left(x_{\lambda^{*}}^{s}\right)$, para $\lambda=\lambda_{*}$ e admita que as suposições $(A 1)-(A 3)$ estejam satisfeitas em um intervalo aberto I contendo $\lambda^{*}$. Então,

$$
\bigcup_{i} W_{\lambda}^{s}\left(x_{\lambda}^{i}\right) \subseteq \partial A_{\lambda}\left(x_{\lambda}^{s}\right)
$$

para todo $\lambda \in I$, onde $x_{\lambda}^{i}, i=1,2, \ldots, k$ são os pontos de equilíbrio hiperbólicos perturbados em $\partial A_{\lambda}\left(x_{\lambda}^{s}\right)$.

O Corolário 6.1.1 permite-nos concluir que, se as suposições $(A 1)-(A 3)$ são satisfeitas para todo $\lambda$ próximo a $\lambda_{*}$, a fronteira da região de estabilidade perturbada $\partial A_{\lambda}\left(x_{\lambda}^{s}\right)$ contém a união das variedades estáveis dos pontos de equilíbrio hiperbólicos perturbados na fronteira $\partial A_{\lambda}\left(x_{\lambda}^{s}\right)$.

No trabalho de Chiang e Chia-Chi Chu (1995), sugere-se erroneamente que $\bigcup_{i} W_{\lambda}^{s}\left(x_{\lambda}^{i}\right)=$ $\partial A_{\lambda}\left(x_{\lambda}^{s}\right)$ para todo $\lambda \in I$, onde $x_{\lambda}^{i}, i=1,2, \ldots, k$ são os pontos de equilíbrio hiperbólicos perturbados em $\partial A_{\lambda}\left(x_{\lambda}^{s}\right)$. Em verdade, o outro lado da inclusão, isto é, $\bigcup_{i} W_{\lambda}^{s}\left(x_{\lambda}^{i}\right) \supseteq$ $\partial A_{\lambda}\left(x_{\lambda}^{s}\right)$ nem sempre é verdadeiro conforme mostra o exemplo a seguir.

Exemplo 6.1.1. Considere o sistema de equações diferenciais

$$
\dot{x}=\frac{-2 x\left(x^{2}+x\right)+2 x\left(x^{2}-1\right)}{\left(x^{2}+1\right)^{2}}+\lambda x
$$

onde $x \in \mathbb{R}$ e $\lambda \in \mathbb{R}$.

O sistema (6.2) possui, para $\lambda_{*}=0$, dois pontos de equilíbrio hiperbólicos, são eles, $x_{\lambda^{*}}^{s}=0$ um ponto de equilíbrio assintoticamente estável e $x_{\lambda^{*}}^{1}=-1 \mathrm{um}$ ponto de equilíbrio hiperbólico instável. O ponto de equilíbrio instável $x_{\lambda^{*}}^{1}$ pertence à fronteira da região de estabilidade $\partial A_{\lambda_{*}}(0)$ e a variedade estável $W_{\lambda^{*}}^{s}(-1)=\{-1\}$ está contida na fronteira da região de estabilidade $\partial A_{\lambda_{*}}(0)$, ver Figura 6.1. Para $\lambda=0,01$, o sistema (6.2) possui três pontos de equilíbrio hiperbólicos, são eles, $x_{\lambda}^{s}=0$ um ponto de equilíbrio assintoticamente estável, $x_{\lambda}^{1}=-0,980$ um ponto de equilíbrio hiperbólico instável e $x_{\lambda}^{2}=$ 6,044 um ponto de equilíbrio hiperbólico instável. Os pontos de equilíbrio 0 e $-0,980$ são os pontos de equilíbrio perturbados e o equilíbrio 6,044 é um novo ponto de equilíbrio que aparece quando o parâmetro é variado. A fronteira da região de estabilidade perturbada $\partial A_{\lambda}(0)$ é composta pelas variedades $W_{\lambda}^{s}(-0,980)=\{-0,980\}$ e $W_{\lambda}^{s}(6,044)=\{6,044\}$, ou seja, $\partial A_{\lambda}(0)=\{-0,980 ; 6,044\}$, ver Figura 6.2. Sendo assim, podemos afirmar que a fronteira da região de estabilidade perturbada $\partial A_{\lambda}(0)$ não está contida na variedade 
estável do ponto de equilíbrio hiperbólico perturbado $W_{\lambda}^{s}(-0,980)=\{-0,980\}$, o que mostra que nem sempre a caracterização da fronteira da região de estabilidade persiste a pequenas variações dos parâmetros.

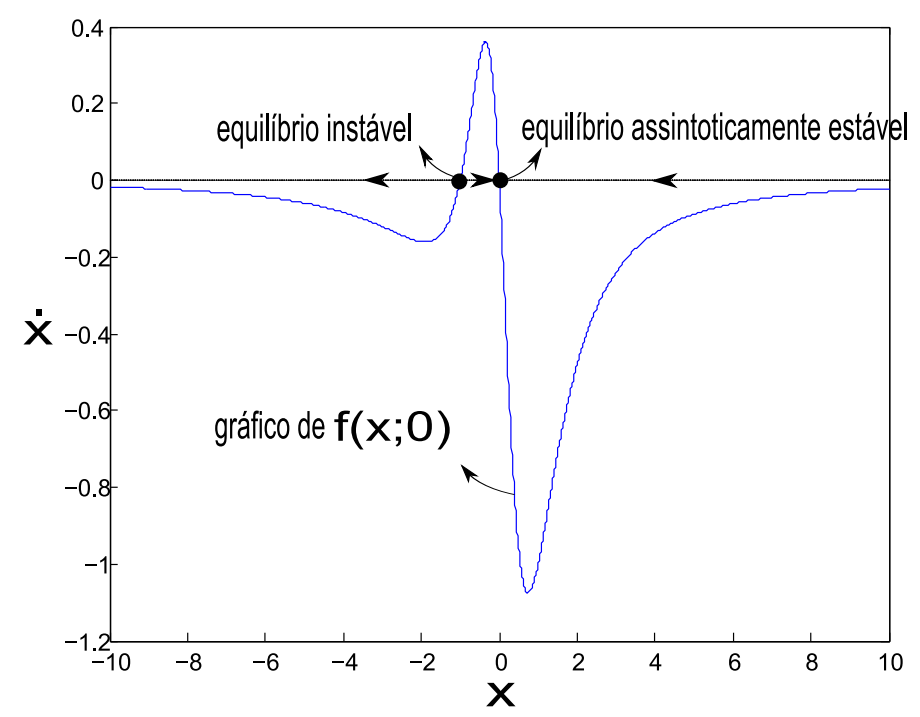

Figura 6.1: Retrato de fase do sistema (6.2) para $\lambda_{*}=0$. O ponto de equilíbrio instável $x_{\lambda^{*}}^{1}=-1$ pertence à fronteira da região de estabilidade do ponto de equilíbrio assintoticamente estável $x_{\lambda^{*}}^{s}=0$.

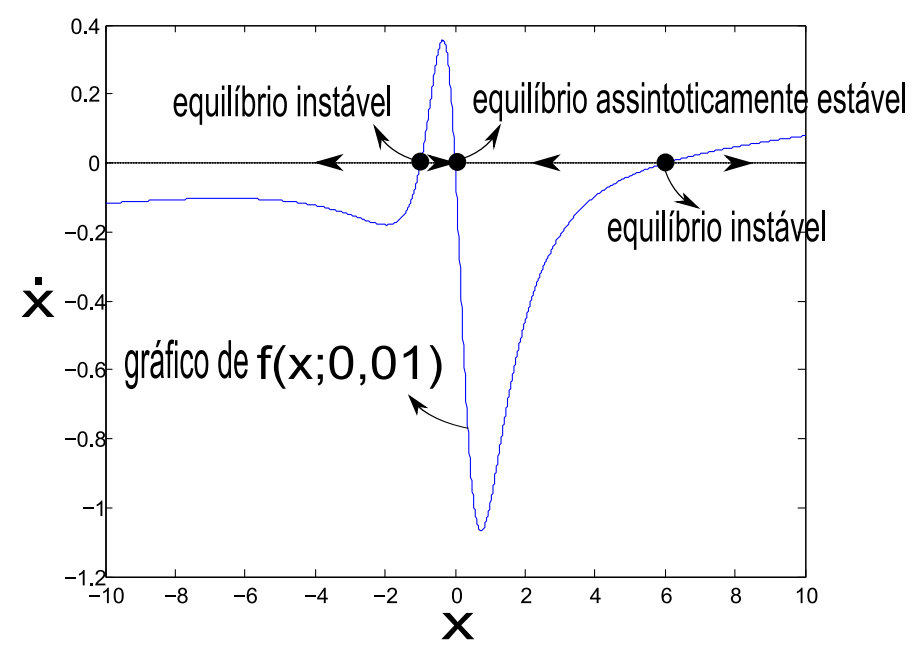

Figura 6.2: Retrato de fase do sistema (6.2) para $\lambda=0,01$. O ponto de equilíbrio instável perturbado $x_{\lambda}^{1}=-0,980$ pertence à fronteira da região de estabilidade do ponto de equilíbrio assintoticamente estável $x_{\lambda}^{s}=0$. E o novo ponto de equilíbrio instável $x_{\lambda}^{2}=6,044$ também pertence à fronteira da região de estabilidade $\partial A_{\lambda}(0)$.

Observação 6.1.1. O sistema (6.2)para $\lambda^{*}=0$ não é estruturalmente estável, pois perturbando o sistema (6.2) com $\lambda$ positivo ocorre mudança no número de equilíbrios do sistema perturbado. Por outro lado, para qualquer $\lambda>0$ o sistema é estruturalmente estável e consequentemente não ocorre mudança no número de equilíbrios do sistema perturbado para variações suficientemente pequenas do parâmetro. 
Explorando o Exemplo 6.1.1, podemos afirmar que, fixando um parâmetro $\lambda^{*}$ do sistema (6.1) e mesmo admitindo que todos os pontos de equilíbrio do sistema $\dot{x}=f\left(x, \lambda^{*}\right)$ são hiperbólicos, quando o parâmetro $\lambda$ varia próximo a $\lambda^{*}$ novos pontos de equilíbrio podem aparecer no sistema perturbado $\dot{x}=f(x, \lambda)$. Esses novos equilíbrios podem aparecer inclusive na fronteira da região de estabilidade perturbada, como visto no Exemplo 6.1.1.

\subsection{Exemplos}

Exibiremos nesta seção dois exemplos que ratificam a persistência dos pontos de equilíbrio hiperbólicos na fronteira da região de estabilidade quando as suposições $(A 1)-(A 3)$ são satisfeitas.

Exemplo 6.2.1. Considere o sistema

$$
\dot{x}=x^{2}-\lambda
$$

onde $x \in \mathbb{R}$ e $\lambda \in \mathbb{R}$.

O sistema (6.3) possui, para $\lambda_{*}=1$, dois pontos de equilíbrio hiperbólicos, são eles, $x_{\lambda^{*}}^{s}=-1 \mathrm{um}$ ponto de equilíbrio assintoticamente estável e $x_{\lambda^{*}}^{1}=1 \mathrm{um}$ ponto de equilíbrio hiperbólico do tipo 1 . O ponto de equilíbrio $x_{\lambda^{*}}^{1}$ pertence à fronteira da região de estabilidade de $x_{\lambda^{*}}^{s}=-1$ e $\partial A_{\lambda_{*}}(-1)=\{1\}$, onde $\{1\}$ é a variedade estável de $x_{\lambda^{*}}^{1}=1$, ver Figura 6.4. Para $\lambda=0,9$, o sistema (6.3) possui dois pontos de equilíbrio hiperbólicos, são eles, $x_{\lambda}^{s}=-0,9487$ um ponto de equilíbrio assintoticamente estável e $x_{\lambda}^{1}=0,9487$ um ponto de equilíbrio hiperbólico do tipo 1 . O ponto de equilíbrio perturbado $x_{\lambda}^{1}=0,9487$ pertence à fronteira da região de estabilidade de $x_{\lambda}^{s}=-0,9487$ de acordo com o Teorema 6.1.2. Além disso, a fronteira da região de estabilidade perturbada $\partial A_{\lambda}(-0,9487)=\{0,9487\}$, onde $\{0,9487\}$ é a variedade estável de $x_{\lambda}^{1}=0,9487$, ver Figura 6.3. Para $\lambda=1,1$, o sistema (6.3) possui dois pontos de equilíbrio hiperbólicos, são eles, $x_{\lambda}^{s}=-1,0488$ um ponto de equilíbrio assintoticamente estável e $x_{\lambda}^{1}=1,0488$ um ponto de equilíbrio hiperbólico do tipo 1 . O ponto de equilíbrio perturbado $x_{\lambda}^{1}=1,0488$ pertence à fronteira da região de estabilidade de $x_{\lambda}^{s}=-1,0488$ de acordo com o Teorema 6.1.2. Além disso, a fronteira da região de estabilidade perturbada $\partial A_{\lambda}(-1,0488)=$ $\{1,0488\}$, onde $\{1,0488\}$ é a variedade estável de $x_{\lambda}^{1}=1,0488$, ver Figura 6.5. Neste exemplo, com a variação do parâmetro $\lambda$ suficientemente próximo do parâmetro $\lambda_{*}=1$, a persistência da caracterização da fronteira da região de estabilidade é verificada.

Exemplo 6.2.2. Considere o sistema

$$
\begin{aligned}
& \dot{x}=2 x+x^{2}+\lambda \\
& \dot{y}=x-y+y^{2}
\end{aligned}
$$




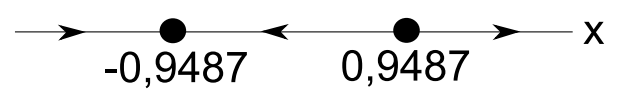

Figura 6.3: Retrato de fase do sistema (6.3) para $\lambda=0,9$. A fronteira da região de estabilidade perturbada $\partial A_{\lambda}(-0,9487)$ do ponto de equilíbrio assintoticamente estável $x_{\lambda}^{s}=-0,9487$ é constituída pelo ponto de equilíbrio hiperbólico perturbado $x_{\lambda}^{1}=0,9487$.

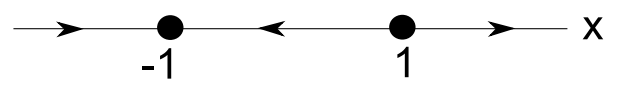

Figura 6.4: Retrato de fase do sistema (6.3) para $\lambda_{*}=1$. A fronteira da região de estabilidade $\partial A_{\lambda_{*}}(-1)$ do ponto de equilíbrio assintoticamente estável $x_{\lambda^{*}}^{s}=-1$ é constituída pelo ponto de equilíbrio hiperbólico $x_{\lambda^{*}}^{1}=1$.

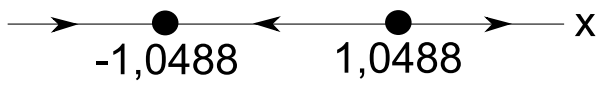

Figura 6.5: Retrato de fase do sistema (6.3) para $\lambda=1,1$. A fronteira da região de estabilidade perturbada $\partial A_{\lambda}(-1,0488)$ do ponto de equilíbrio assintoticamente estável $x_{\lambda}^{s}=-1,0488$ é constituída pelo ponto de equilíbrio hiperbólico perturbado $x_{\lambda}^{1}=1,0488$.

onde $(x ; y) \in \mathbb{R}^{2}$.

O sistema (6.4) possui, para $\lambda_{*}=0$, quatro pontos de equilíbrio hiperbólicos, são eles, $x_{\lambda^{*}}^{s}=(-2 ;-1)$ um ponto de equilíbrio hiperbólico assintoticamente estável, $x_{\lambda^{*}}^{1}=(0 ; 0)$ um ponto de equilíbrio hiperbólico do tipo $1, x_{\lambda^{*}}^{2}=(-2 ; 2)$ um ponto de equilíbrio hiperbólico do tipo 1 e $x_{\lambda^{*}}^{3}=(0 ; 1)$ um ponto de equilíbrio hiperbólico do tipo 2 . Os pontos de equilíbrio $x_{\lambda^{*}}^{1}, x_{\lambda^{*}}^{2}$ e $x_{\lambda^{*}}^{3}$ pertencem à fronteira da região de estabilidade de $x_{\lambda^{*}}^{s}=(-2 ;-1)$ e $\partial A_{\lambda_{*}}(-2 ;-1)$ é a união das variedades estáveis dos pontos de equilíbrio $(-2 ; 2)$, $(0 ; 1)$ e $(0 ; 0)$, ver Figura 6.7. Para $\lambda=-0,1$, o sistema (6.4) possui quatro pontos de equilíbrio hiperbólicos, são eles, $x_{\lambda}^{s}=(-2,0488 ;-1,0162)$ um ponto de equilíbrio hiperbólico assintoticamente estável, $x_{\lambda}^{1}=(-2,0488 ; 2,0162)$ um ponto de equilíbrio hiperbólico do tipo $1, x_{\lambda}^{2}=(0,0488 ; 0,0514)$ um ponto de equilíbrio hiperbólico do tipo 1 e $x_{\lambda}^{3}=(0,0488 ; 0,9485)$ um ponto de equilíbrio hiperbólico do tipo 2. Os pontos de equilíbrio $x_{\lambda}^{1}, x_{\lambda}^{2}$ e $x_{\lambda}^{3}$ pertencem à fronteira da região de estabilidade de $x_{\lambda}^{s}=(-2,0488 ;-1,0162)$ de acordo com o Teorema 6.1.2. Além disso, a fronteira da região de estabilidade perturbada $\partial A_{\lambda}(-2,0488 ;-1,0162)$ é a união das variedades estáveis dos pontos de equilíbrio perturbados $(-2,0488 ; 2,0162),(0,0488 ; 0,0514)$ e $(0,0488 ; 0,9485)$, ver Figura 6.6. Para $\lambda=0,1$, o sistema (6.4) possui quatro pontos de equilíbrio hiperbólicos, são eles, $x_{\lambda}^{s}=(-1,9487 ;-0,9828)$ um ponto de equilíbrio hiperbólico assintoticamente estável, $x_{\lambda}^{1}=(-1,9487 ; 1,98282)$ um ponto de equilíbrio hiperbólico do tipo $1, x_{\lambda}^{2}=(-0,0513 ;-0,0489)$ um ponto de equilíbrio hiperbólico do tipo 1 e $x_{\lambda}^{3}=(-0,0513 ; 1,0489)$ um ponto de equilíbrio hiperbólico do tipo 2. Os pontos de equilíbrio $x_{\lambda}^{1}, x_{\lambda}^{2}$ e $x_{\lambda}^{3}$ pertencem à fronteira da região de estabilidade de $x_{\lambda}^{s}=(-1,9487 ;-0,9828)$ de acordo com o Teorema 6.1.2. Além disso, a fron- 
teira da região de estabilidade perturbada $\partial A_{\lambda}(-1,9487 ;-0,9828)$ é a união das variedades estáveis dos pontos de equilíbrio $(-1,9487 ; 1,98282),(-0,0513 ;-0,0489)$ e $(-0,0513 ; 1,0489)$, ver Figura 6.8. Neste exemplo, com a variação do parâmetro $\lambda$ suficientemente próximo do parâmetro $\lambda_{*}=0$, a persistência da caracterização da fronteira da região de estabilidade é verificada.

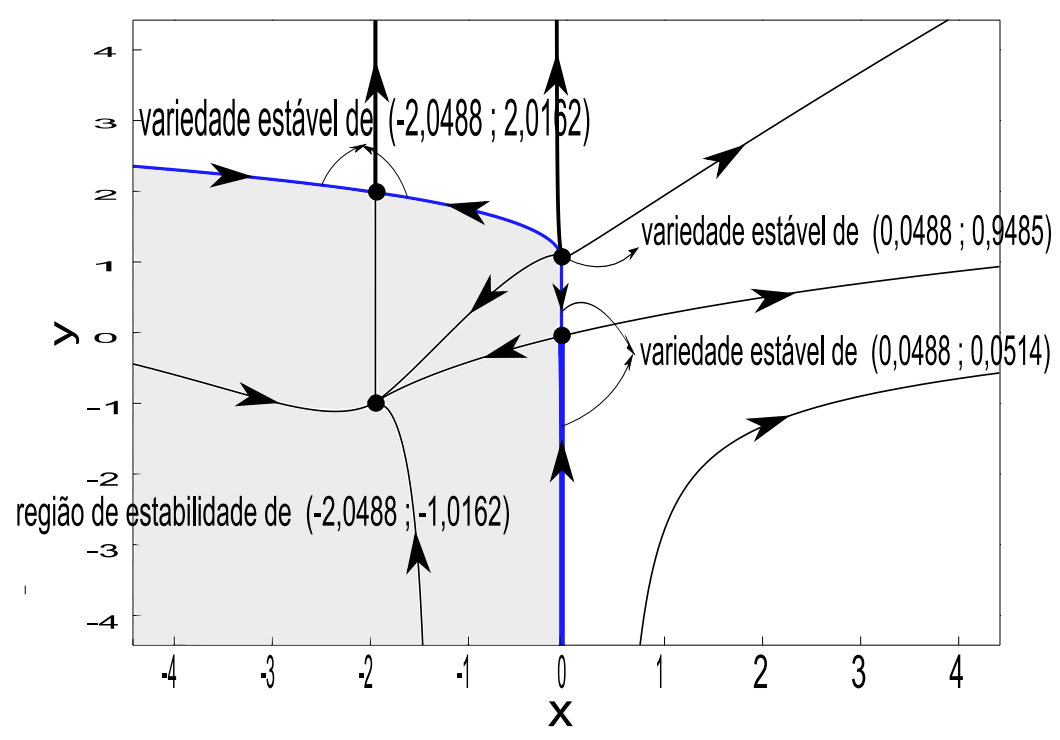

Figura 6.6: Retrato de fase do sistema (6.4) para $\lambda=-0,1$. A fronteira da região de estabilidade perturbada $\partial A_{\lambda}(-2,0488 ;-1,0162)$ é a união das variedades estáveis dos pontos de equilíbrio $(-2,0488 ; 2,0162),(0,0488 ; 0,0514)$ e $(0,0488 ; 0,9485)$, as curvas em azul.

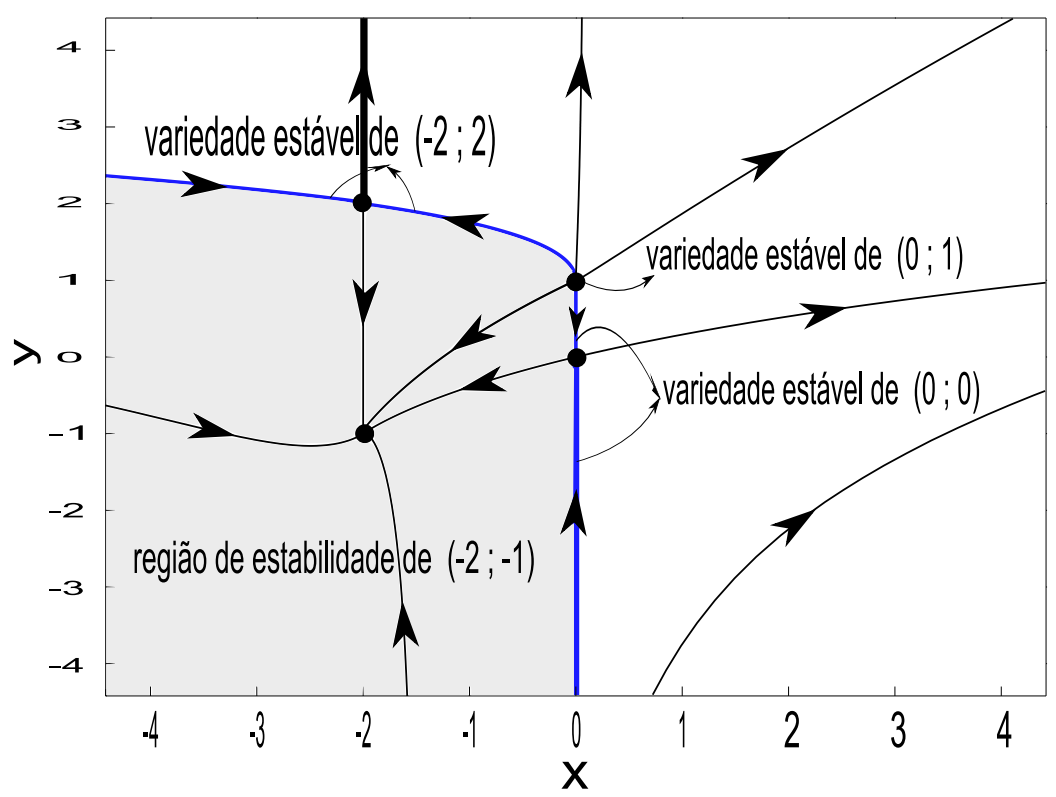

Figura 6.7: Retrato de fase do sistema (6.4) para $\lambda_{*}=0$. A fronteira da região de estabilidade $\partial A_{\lambda_{*}}(-2 ;-1)$ é a união das variedades estáveis dos pontos de equilíbrio $(-2 ; 2)$, $(0 ; 1)$ e $(0 ; 0)$, as curvas em azul. 


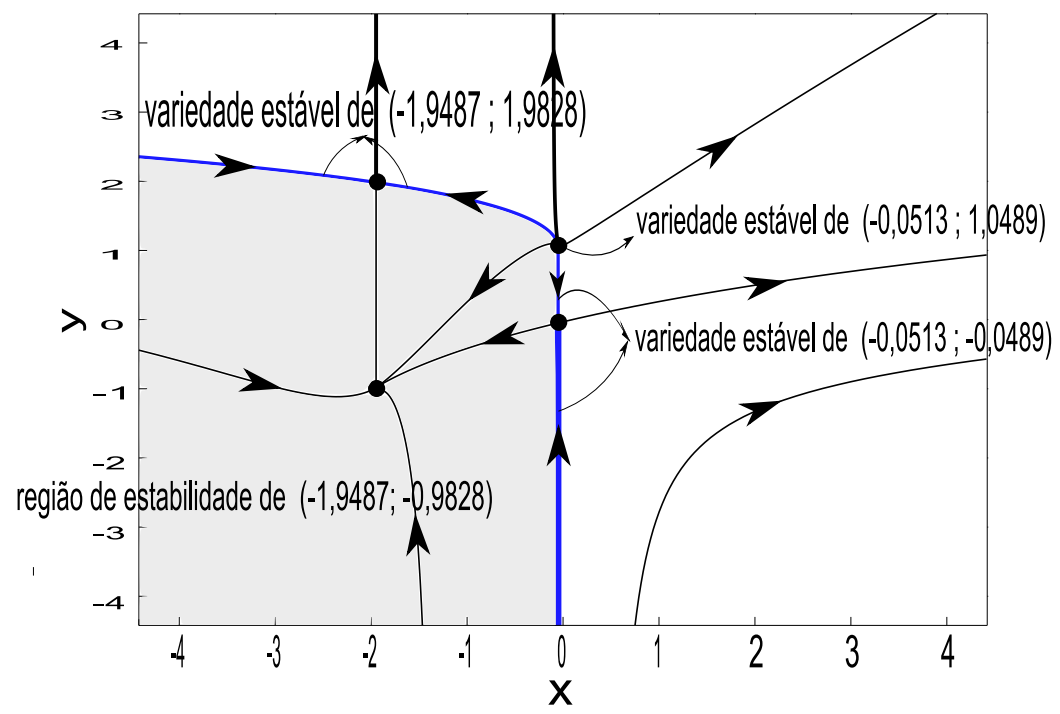

Figura 6.8: Retrato de fase do sistema (6.4) para $\lambda=0,1$. A fronteira da região de estabilidade perturbada $\partial A_{\lambda}(-1,9487 ;-0,9828)$ é a união das variedades estáveis dos pontos de equilíbrio $(-1,9487 ; 1,98282),(-0,0513 ;-0,0489)$ e $(-0,0513 ; 1,0489)$, as curvas em azul. 


\section{BIFURCAÇÃO A UM PARÂMETRO}

Neste capítulo, faremos uma breve revisão sobre a teoria de bifurcação para uma família de equações diferenciais dependendo de um parâmetro escalar. Na Seção 7.1, introduziremos o conceito de estabilidade estrutural e parâmetro de bifurcação. Na Seção 7.2, exploraremos a teoria de bifurcação sela-nó, visto que no Capítulo 8, estudaremos o comportamento da fronteira da região de estabilidade próximo a um parâmetro de bifurcação sela-nó. Para maiores detalhes dos assuntos tratados neste capítulo, ver (PALIS; MELO, 1977), (PALIS, 1969), (SMITH, 1991), (WIGGINS, 2003), (GUCKENHEIMER; HOLMES, 1983) e (SOTOMAYOR, 1973).

\subsection{Estabilidade Estrutural}

Iniciaremos a seção definindo o conceito de equivalência topológica.

Definição 7.1.1. (Equivalência Topológica) Sejam $f, g: \mathbb{R}^{n} \rightarrow \mathbb{R}^{n}$ dois campos vetoriais de classe $C^{1}$. Dizemos que $f$ e g são topologicamente equivalentes se existe um homeomorfismo $h: \mathbb{R}^{n} \longrightarrow \mathbb{R}^{n}$ que leva órbitas de $f$ em órbitas de g preservando a orientação das órbitas.

O homeomorfismo $h$ da Definição 7.1.1 é chamado de equivalência topológica entre os campos vetoriais $f$ e $g$.

Exibiremos a seguir um exemplo de equivalência topológica.

Exemplo 7.1.1. (SOTOMAYOR, 1979) Considere os campos $f(x, y)=\left(x,-y+x^{3}\right) e$ $g(x, y)=(x,-y)$ em $\mathbb{R}^{2}$. Dada uma condição inicial $\left(x_{0}, y_{0}\right) \in \mathbb{R}^{2}$ de $f$ a solução que passa por essa condição inicial é dada por $\varphi\left(t,\left(x_{0}, y_{0}\right)\right)=\left(x_{0} e^{t},\left(y_{0}-\frac{x_{0}^{3}}{4}\right) e^{-t}+\frac{x_{0}^{3}}{4} e^{3 t}\right)$ onde $t \in \mathbb{R}$. Para o campo g a solução que passa por $\left(x_{0}, y_{0}\right) \in \mathbb{R}^{2}$ é dada por $\psi\left(t,\left(x_{0}, y_{0}\right)\right)=$ $\left(x_{0} e^{t}, y_{0} e^{-t}\right)$ onde $t \in \mathbb{R}$. Sem maiores dificuldades, pode se verificar que, $h(\psi(t, p))=$ $\varphi(t, h(p))$ para qualquer $p \in \mathbb{R}^{2}$ onde $h:(x, y) \longrightarrow\left(x, y+\frac{x^{3}}{4}\right)$. Em particular, podemos afirmar que h leva órbitas de g em órbitas de $f$.

A Figura 7.1 exibe o retrato de fase dos campos vetoriais $f$ e $g$ do Exemplo 7.1.1. 


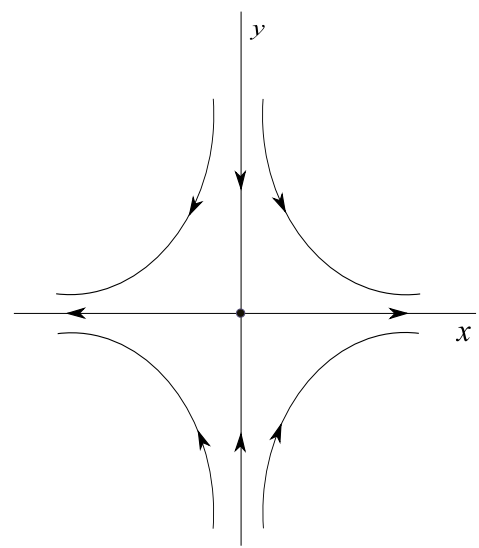

retrato de fase de $\mathrm{g}$

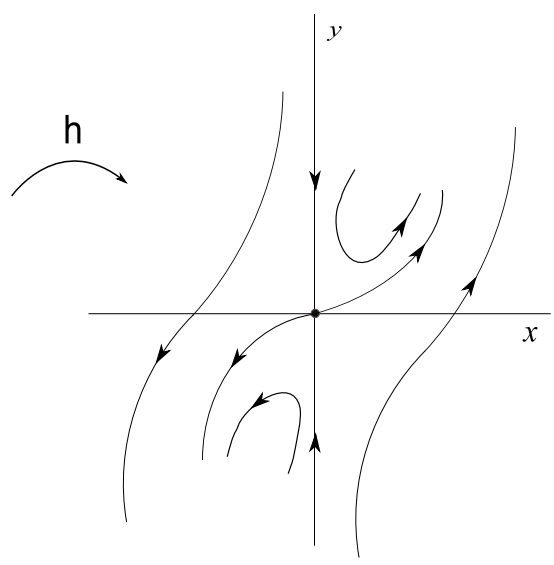

retrato de fase de $f$

Figura 7.1: Retrato de fase dos campos vetoriais $f$ e $g$.

O teorema a seguir, cuja demonstração é imediata, exprime uma relação entre os pontos de equilíbrio, conjunto $\omega$-limite e $\alpha$-limite de dois campos vetoriais que possuem uma equivalência topológica.

Teorema 7.1.1. (PALIS; MELO, 1977) Seja h um equivalência topológica entre os campos vetoriais $f, g: \mathbb{R}^{n} \rightarrow \mathbb{R}^{n}$ de classe $C^{1}$. Então

(i) $x^{*}$ é um ponto de equilíbrio de $f$ se, e somente se, $h\left(x^{*}\right)$ é um ponto de equilíbrio de g; (ii) a imagem do $\omega$-limite da solução começando na condição inicial p via hé o $\omega$-limite da solução começando na condição inicial $h(p)$; analogamente para o $\alpha$-limite.

O estudo de mudanças na estrutura qualitativa do fluxo de um equação diferencial quando parâmetros são variados é chamada teoria da bifurcação. Como estamos interessados em estudar bifurcação a um parâmetro escalar, considere a família de equações diferenciais

$$
\dot{x}=f(x, \lambda)
$$

com $x \in \mathbb{R}^{n}, \lambda \in \mathbb{R}$ e $f: \mathbb{R}^{n} \times \mathbb{R} \longrightarrow \mathbb{R}^{n}$ um campo vetorial de classe $C^{r}$, com $r \geqslant 2$. Para cada $\lambda$ fixo, definamos o campo vetorial $f_{\lambda}()=.f(., \lambda)$ e $\varphi_{\lambda}(t, x)$ denota a solução de $\dot{x}=f_{\lambda}(x)$ passando por $x$ no tempo $t=0$.

Estudaremos daqui em diante os conceitos que envolvem a teoria de bifurcação a um parâmetro.

Definição 7.1.2. (SMITH, 1991) O campo vetorial $f_{\lambda_{0}}$, para uma parâmetro $\lambda_{0}$ fixo, é dito ser estruturalmente estável, se existe $\varepsilon>0$ tal que $f_{\lambda}$ é topologicamente equivalente a $f_{\lambda_{0}}$ para todo $\lambda$ satisfazendo $\left|\lambda-\lambda_{0}\right|<\varepsilon$.

A seguir, apresenta-se a definição de parâmetro de bifurcação.

Definição 7.1.3. Dizemos que $\lambda_{0}$ é um parâmetro de bifurcação, se o campo vetorial $f_{\lambda_{0}}$ não for estruturalmente estável. 
Exemplo 7.1.2. Considere a família de equações diferenciais

$$
\dot{x}=\lambda-x^{2}
$$

onde $x \in \mathbb{R}$ e $\lambda \in \mathbb{R}$.

Para cada $\lambda$ fixo, temos que $f_{\lambda}(x)=f(x, \lambda)=\lambda-x^{2}$. Para $\lambda_{0}=0$ o campo $f_{\lambda_{0}}$ tem um ponto de equilíbrio não hiperbólico, para $\lambda>0$ o campo $f_{\lambda_{0}}$ tem dois pontos de equilíbrio e para $\lambda<0$ o campo $f_{\lambda_{0}}$ não tem nenhum ponto de equilíbrio, ver Figura 7.2. Logo, $f_{\lambda_{0}}$ não é estruturalmente estável, isto é, $\lambda_{0}=0$ é um parâmetro de bifurcação de $\lambda$.

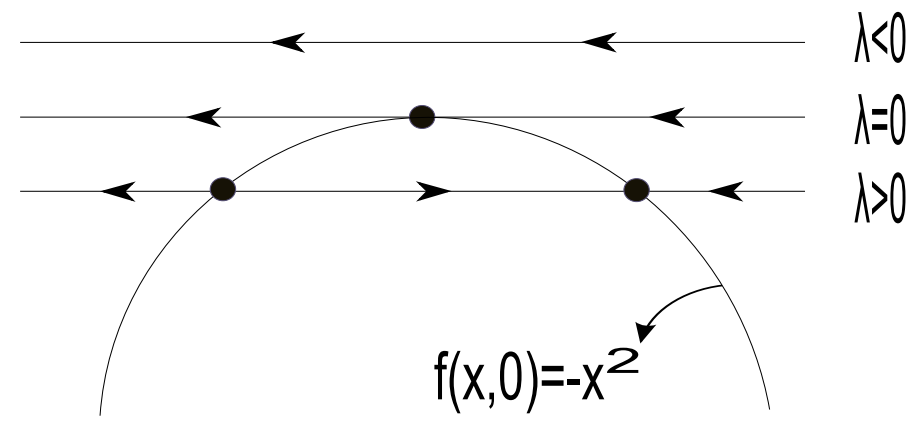

Figura 7.2: Retratos de fase do sistema (7.2).

\subsection{Bifurcação sela-nó}

Nesta tese, estudaremos um tipo particular de bifurcação que é denominada bifurcação sela-nó. Uma breve revisão da teoria de bifurcação sela-nó será apresentada nesta seção. Em uma família de equação diferenciais dependendo de um parâmetro escalar, essa bifurcação é genérica. Começaremos a seção definindo o conceito de bifurcação sela-nó.

Definição 7.2.1. (Ponto de Bifurcação Sela-Nó) Seja $x_{\lambda_{0}} \in \mathbb{R}^{n}$ um ponto de equilíbrio não hiperbólico de (7.1), para um parâmetro fixo $\lambda=\lambda_{0}$. Dizemos que $\left(x_{\lambda_{0}}, \lambda_{0}\right)$ é um ponto de bifurcação sela-nó se as seguintes condições são satisfeitas:

(SN1) $D_{x} f_{\lambda_{0}}\left(x_{\lambda_{0}}\right)$ tem um único autovalor simples igual a 0 com $v$ sendo um autovetor à direita e $w$ à esquerda.

(SN2) $w\left(D_{x}^{2} f_{\lambda_{0}}\left(x_{\lambda_{0}}\right)(v, v)\right) \neq 0$.

$(S N 3) w\left(\left(\partial f_{\lambda} / \partial \lambda\right)\left(x_{\lambda_{0}}, \lambda_{0}\right)\right) \neq 0$.

Observação 7.2.1. Dado um ponto de de bifurcação sela-nó $\left(x_{\lambda_{0}}, \lambda_{0}\right)$ de (7.1), para um parâmetro fixo $\lambda=\lambda_{0}$, o ponto de equilíbrio não hiperbólico $x_{\lambda_{0}}$ é um ponto de equilíbrio sela-nó de (7.1), para $\lambda=\lambda_{0}$ (vide Definição 5.1.1). 
O teorema a seguir descreve o comportamento dinâmico do sistema (7.1) próximo a um ponto de bifurcação sela-nó, sua demonstração pode ser encontrada em (SOTOMAYOR, 1973).

Teorema 7.2.1. (SOTOMAYOR, 1973) Seja $\left(x_{\lambda_{0}}, \lambda_{0}\right)$ um ponto de bifurcação sela-nó de (7.1). Então existe uma vizinhança $N$ de $x_{\lambda_{0}}$ e $\delta>0$ tal que, dependendo dos sinais das expressões em (SN2) e (SN3), não existe ponto de equilíbrio em $N$ quando $\lambda \in\left(\lambda_{0}-\right.$ $\left.\delta, \lambda_{0}\right)\left[\lambda \in\left(\lambda_{0}, \lambda_{0}+\delta\right)\right]$ e existem dois pontos de equilíbrio em $N$ para cada $\lambda \in\left(\lambda_{0}, \lambda_{0}+\right.$ $\delta)\left[\lambda \in\left(\lambda_{0}-\delta, \lambda_{0}\right)\right]$. Os dois pontos de equilíbrio em $N$ são hiperbólicos do tipo $k e$ $k+1, k \in \mathbb{N}$, respectivamente. Além disso, a variedade estável do ponto de equilíbrio hiperbólico tipo $k$ intercepta a variedade instável do ponto de equilíbrio hiperbólico tipo $k+1$ ao longo de uma variedade unidimensional.

\subsubsection{Bifurcação sela-nó do tipo zero}

Assim como os equilíbrios sela-nó, pontos de bifurcação sela-nó podem ser classificados em tipos de acordo com o número de autovalores de $D_{x} f_{\lambda_{0}}\left(x_{\lambda_{0}}\right)$ com parte real positiva.

Definição 7.2.2. (Tipo de Bifurcação Sela-Nó) Um ponto de bifurcação sela-nó $\left(x_{\lambda_{0}}, \lambda_{0}\right)$ de (7.1) é chamado um ponto de bifurcação sela-nó do tipo $k$ se $D_{x} f_{\lambda_{0}}\left(x_{\lambda_{0}}\right)$ tem $k$ autovalores com parte real positiva e $n-k-1$ com parte real negativa, $k \in \mathbb{N}$.

Neste texto, estudaremos apenas os pontos de bifurcação sela-nó do tipo zero. Sendo assim, alguns resultados especializados para pontos de bifurcação sela-nó do tipo zero são desenvolvidos.

O teorema a seguir descreve o comportamento dinâmico do sistema (7.1) próximo a um ponto de equilíbrio sela-nó do tipo zero.

Teorema 7.2.2. (SOTOMAYOR, 1973) (Birfucação Sela-Nó do Tipo Zero) Seja $\left(x_{\lambda_{0}}, \lambda_{0}\right)$ um ponto de bifurcação sela-nó do tipo zero de (7.1). Então existe uma vizinhança $N$ de $x_{\lambda_{0}}$ e $\delta>0$ tal que, dependendo dos sinais das expressões em (SN2) e (SN3), não existe ponto de equilíbrio em $N$ quando $\lambda \in\left(\lambda_{0}-\delta, \lambda_{0}\right)\left[\lambda \in\left(\lambda_{0}, \lambda_{0}+\delta\right)\right]$ e existem dois pontos de equilíbrio $y_{\lambda}^{s}$ e $y_{\lambda}^{u}$ em $N$ para cada $\lambda \in\left(\lambda_{0}, \lambda_{0}+\delta\right)\left[\lambda \in\left(\lambda_{0}-\delta, \lambda_{0}\right)\right]$. Os dois pontos de equilíbrio em $N$ são hiperbólicos, mais especificamente $y_{\lambda}^{s}$ é do tipo 0 e $y_{\lambda}^{u}$ é do tipo 1. Além disso, a variedade estável do ponto de equilíbrio do tipo 0 intercepta a variedade instável do ponto de equilíbrio do tipo 1 ao longo de uma variedade unidimensional.

Observação 7.2.2. Pelo Teorema 7.2.2 podemos afirmar que, se $\left(x_{\lambda_{0}}, \lambda_{0}\right)$ é um ponto de bifurcação sela-nó do tipo zero de (7.1), então o campo vetorial $f_{\lambda_{0}}$ não é estruturalmente estável na vizinhança $N$. Ao longo do texto, o parâmetro de bifurcação $\lambda_{0}$ será chamado de parâmetro de bifurcação sela-nó do tipo zero. 
Neste trabalho, admitimos, sem perda de generalidade, que no Teorema 7.2.2 os dois pontos de equilíbrios existem em $N$ para $\lambda \in\left(\lambda_{0}-\delta, \lambda_{0}\right)$ e não existe ponto de equilíbrio em $N$ para $\lambda \in\left(\lambda_{0}, \lambda_{0}+\delta\right)$. A Figura 7.3 ilustra o Teorema 7.2.2.

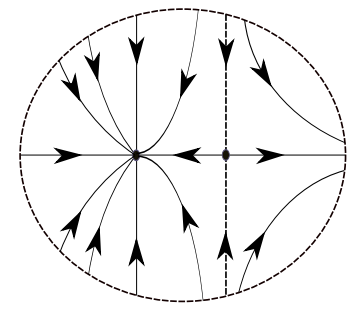

$\lambda<\lambda_{0}$

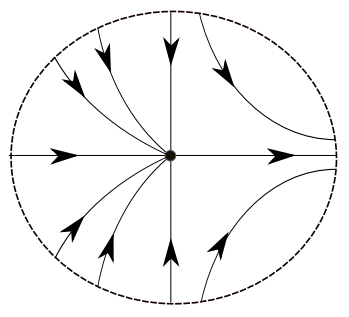

$\lambda=\lambda_{0}$

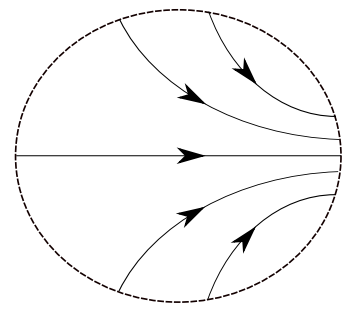

$\lambda>\lambda_{0}$

Figura 7.3: Retrato de fase do sistema (7.1) na vizinhança $N$ do Teorema 7.2.2 para $\lambda \in$ $\left(\lambda_{0}-\delta, \lambda_{0}+\delta\right)$. Para $\lambda<\lambda_{0}$, o sistema (7.1) tem dois pontos de equilíbrio hiperbólicos, um ponto de equilíbrio do tipo 0 e um do tipo 1 . Em $\lambda=\lambda_{0}$, o sistema (7.1) tem um único ponto de equilíbrio $x_{\lambda_{0}}$ que é um ponto de equilíbrio sela-nó do tipo zero. Para $\lambda>\lambda_{0}, o$ sistema (7.1) não tem ponto de equilíbrio em $N$.

Conhecendo o comportamento dinâmico do sistema próximo a um ponto de bifurcação sela-nó do tipo zero $\left(x_{\lambda_{0}}, \lambda_{0}\right)$, qualquer vizinhança $U \subseteq N$ de $x_{\lambda_{0}}$ pode ser decomposta em subconjuntos $U^{+}$e $U^{-}$, da seguinte maneira:

Para $\lambda=\lambda_{0}$, definimos

$$
\begin{gathered}
U_{\lambda_{0}}^{-}:=\left\{q \in\left(U-W_{l o c, \lambda_{0}}^{s}\left(x_{\lambda_{0}}\right)\right): \varphi_{\lambda_{0}}(t, q) \rightarrow x_{\lambda_{0}} \text { quando } t \rightarrow \infty\right\} \\
U_{\lambda_{0}}^{+}:=U-U_{\lambda_{0}}^{-}
\end{gathered}
$$

e para $\lambda \in\left(\lambda_{0}-\delta, \lambda_{0}\right)$, definimos

$$
\begin{gathered}
U_{\lambda}^{-}:=\left\{q \in U: \varphi_{\lambda}(t, q) \rightarrow y_{\lambda}^{s} \text { quando } t \rightarrow \infty\right\} \\
U_{\lambda}^{+}:=U-U_{\lambda}^{-} .
\end{gathered}
$$

A Figura 7.4 ilustra o conjunto $U_{\lambda}^{-}$. 


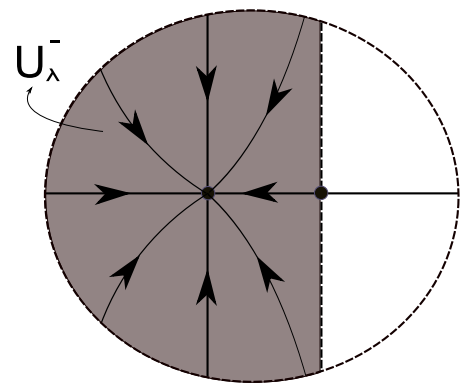

Figura 7.4: Vizinhança $U$ de um ponto de equilíbrio sela-nó do tipo zero $x_{\lambda_{0}}$ para $\lambda<\lambda_{0}$. A parte cinza da figura corresponde ao conjunto $U_{\lambda}^{-}$. 


\section{BIFURCAÇÃO SELA-NÓ DO TIPO ZERO NA FRON- TEIRA DA REGIÃO DE ESTABILIDADE}

Caracterizamos, no Capítulo 5, a fronteira da região de estabilidade de um ponto de equilíbrio assintoticamente estável na presença de um ponto de equilíbrio sela-nó do tipo zero na fronteira. Neste capítulo, mais especificamente na Seção 8.1, mostraremos o comportamento da fronteira da região de estabilidade na vizinhança de um ponto de equilíbrio sela-nó do tipo zero, através deste comportamento local verificaremos que mudanças drásticas podem ocorrer na região de estabilidade e fronteira da região de estabilidade quando parâmetros são variados próximo a um parâmetro de bifurcação sela-nó do tipo zero. Exibiremos também na Seção 8.1 uma caracterização global da fronteira da região de estabilidade próximo a um parâmetro de bifurcação sela-nó do tipo zero. Na última seção exibiremos exemplos que ilustram os resultados da Seção 8.1.

\subsection{Comportamento da fronteira da região de estabilidade na pre- sença de um ponto de equilíbrio sela-nó do tipo zero}

Começaremos esta seção apresentando um resultado auxiliar que garante, a existência de uma vizinhança suficientemente pequena de um ponto de equilíbrio sela-nó do tipo zero $x_{\lambda_{0}} \in \partial A_{\lambda_{0}}\left(x_{\lambda_{0}}^{s}\right)$ que é disjunta das variedades estáveis de todos os pontos de equilíbrio que estão na fronteira $\partial A_{\lambda_{0}}\left(x_{\lambda_{0}}^{s}\right)$.

Lema 8.1.1. Seja $x_{\lambda_{0}}$ um ponto de equilíbrio sela-nó do tipo zero na fronteira da região de estabilidade $\partial A_{\lambda_{0}}\left(x_{\lambda_{0}}^{s}\right)$ de um ponto de equilíbrio hiperbólico assintoticamente estável $x_{\lambda_{0}}^{s}$ de (7.1) para $\lambda=\lambda_{0}$. Se $x_{\lambda_{0}}^{i}, i=1, \ldots, k$ são os pontos de equilíbrio hiperbólicos em $\partial A_{\lambda_{0}}\left(x_{\lambda_{0}}^{s}\right)$, então existe uma vizinhança $U$ de $x_{\lambda_{0}}$ tal que $U \cap W_{\lambda_{0}}^{s}\left(x_{\lambda_{0}}^{i}\right)=\emptyset$ para todo $i=1, \ldots, k$.

Demonstração. Sejam $N$ uma vizinhança de $x_{\lambda_{0}}$ e $x_{\lambda_{0}}^{i}, i=1, \ldots, k$ os pontos de equilíbrio em $\partial A_{\lambda_{0}}\left(x_{\lambda_{0}}^{s}\right)$. Considere uma partição de $\{1, \ldots, k\}=\{1, \ldots, s\} \cup\{s+1, \ldots, k\}$, onde $l \in$ $\{1, . ., s\}$ se $N \cap W_{\lambda_{0}}^{s}\left(x_{\lambda_{0}}^{l}\right)=\emptyset$ e $j \in\{s+1, . ., k\}$ se $N \cap W_{\lambda_{0}}^{s}\left(x_{\lambda_{0}}^{j}\right) \neq \emptyset$. Se $\{s+1, \ldots, k\}=\emptyset$, o lema está provado. Suponha que $\{s+1, \ldots, k\} \neq \emptyset$. Desde que $N_{\lambda_{0}}^{-} \cap W_{\lambda_{0}}^{s}\left(x_{\lambda_{0}}^{j}\right)=\emptyset$ então 
$N_{\lambda_{0}}^{+} \cap W_{\lambda_{0}}^{s}\left(x_{\lambda_{0}}^{j}\right) \neq \emptyset$ para todo $j \in\{s+1, . ., k\}$. A vizinhança $N$ pode ser escolhida suficientemente pequena de tal modo que $\overline{N_{\lambda_{0}}^{+} \cap W_{\lambda_{0}}^{s}\left(x_{\lambda_{0}}^{j}\right)} \subset W_{\lambda_{0}}^{s}\left(x_{\lambda_{0}}^{j}\right)$ para todo $j \in\{s+1, . ., k\}$ e $\overline{N \cap W_{\lambda_{0}}^{s}\left(x_{\lambda_{0}}\right)} \subset W_{\lambda_{0}}^{s}\left(x_{\lambda_{0}}\right)$. Como $W_{\lambda_{0}}^{s}\left(x_{\lambda_{0}}^{j}\right) \cap W_{\lambda_{0}}^{s}\left(x_{\lambda_{0}}\right)=\emptyset$, então $\overline{N_{\lambda_{0}}^{+} \cap W_{\lambda_{0}}^{s}\left(x_{\lambda_{0}}^{j}\right)}$ é disjunto de $\overline{N \cap W_{\lambda_{0}}^{s}\left(x_{\lambda_{0}}\right)}$ para todo $j \in\{s+1, . ., k\}$ e estes são conjuntos fechados, logo pelo Teorema 2.1.6 podemos afirmar que existem conjuntos abertos disjuntos $U_{j}$ e $V_{j}$, contendo $\overline{N_{\lambda_{0}}^{+} \cap W_{\lambda_{0}}^{s}\left(x_{\lambda_{0}}^{j}\right)}$ e $\overline{N \cap W_{\lambda_{0}}^{s}\left(x_{\lambda_{0}}\right)}$, respectivamente. Considere $U=\bigcap_{j} V_{j} \cap N$. Como $x_{\lambda_{0}} \in U$, basta mostrar que $U \cap W_{\lambda_{0}}^{s}\left(x_{\lambda_{0}}^{j}\right)=\emptyset$ para todo $j \in\{s+1, . ., k\}$. Suponha, por absurdo, que exista algum $p \in U \cap W_{\lambda_{0}}^{s}\left(x_{\lambda_{0}}^{j}\right)$. Como $U \subset N$, então $p \in\left(N_{\lambda_{0}}^{+} \cap W_{\lambda_{0}}^{s}\left(x_{\lambda_{0}}^{j}\right)\right) \subset$ $\overline{N_{\lambda_{0}}^{+} \cap W_{\lambda_{0}}^{s}\left(x_{\lambda_{0}}^{j}\right)} \subset U_{j}$. Por outro lado, como $U \subset V_{j}$ temos que $p \in U_{j} \cap V_{j}$ levandonos a um absurdo, pois $U_{j}$ e $V_{j}$ são disjuntos. Logo, $U \cap W_{\lambda_{0}}^{s}\left(x_{\lambda_{0}}^{j}\right)=\emptyset$ para todo $j \in$ $\{s+1, \ldots, k\}$. Se $\{1, \ldots, s\}=\emptyset$ a demonstração acabou, caso contrário, se existir índice $l$ tal que $N \cap W_{\lambda_{0}}^{s}\left(x_{\lambda_{0}}^{l}\right)=\emptyset$ e sabendo que $U \subset N$ podemos afirmar que $U \cap W_{\lambda_{0}}^{s}\left(x_{\lambda_{0}}^{l}\right)=\emptyset$ para todo $l \in\{1, \ldots, s\}$. Portanto, $U \cap W_{\lambda_{0}}^{s}\left(x_{\lambda_{0}}^{i}\right)=\emptyset$ para todo $i \in\{1, \ldots, k\}$ e o lema está provado.

Como consequência do Teorema 5.5.4 e Lema 8.1.1, obtemos o seguinte corolário:

Corolário 8.1.1. Seja $x_{\lambda_{0}}$ um ponto de equilíbrio sela-nó do tipo zero na fronteira da região de estabilidade $\partial A_{\lambda_{0}}\left(x_{\lambda_{0}}^{s}\right)$ de um ponto de equilíbrio hiperbólico assintoticamente estável $x_{\lambda_{0}}^{s}$ de (7.1) para $\lambda=\lambda_{0}$. Suponha que $\left(A 1^{\prime}\right)$, (A3) e (A4) sejam satisfeitas para $\lambda=\lambda_{0}$ e o número de pontos de equilíbrio na fronteira $\partial A_{\lambda_{0}}\left(x_{\lambda_{0}}^{s}\right)$ seja finito. Então existe uma vizinhança $U$ de $x_{\lambda_{0}}$ tal que $\left(U_{\lambda_{0}}^{+}-W_{\lambda_{0}}^{s}\left(x_{\lambda_{0}}\right)\right) \subset A_{\lambda_{0}}\left(x_{\lambda_{0}}^{s}\right)$.

A Figura 8.1 ilustra o Corolário 8.1.1.

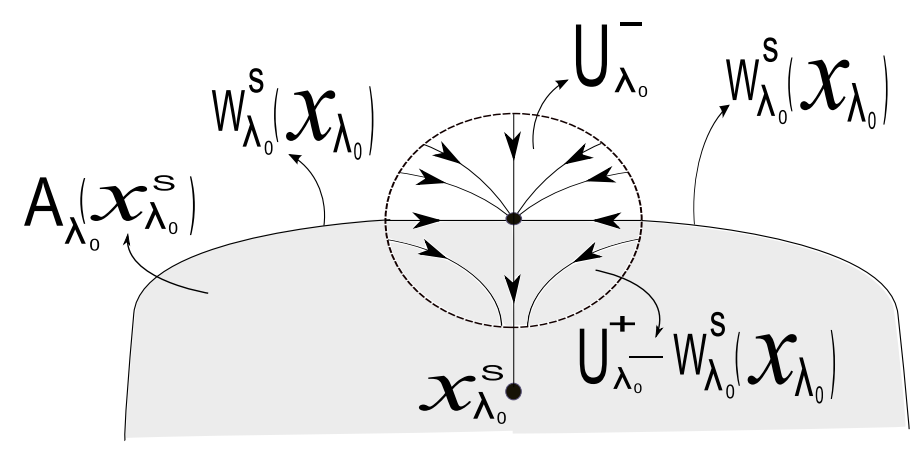

Figura 8.1: Comportamento da vizinhança $U$ de um ponto de equilíbrio sela-nó do tipo zero $x_{\lambda_{0}}$ que está na fronteira da região de estabilidade de um ponto de equilíbrio assintoticamente estável $x_{\lambda_{0}}^{s}$.

O Teorema 6.1.2 garante, sob algumas hipóteses, que os pontos de equilíbrio hiperbólicos na fronteira da região de estabilidade persistem na fronteira. Dentre as hipóteses consideradas no Teorema 6.1.2, uma delas é que todos os pontos de equilíbrio na fronteira sejam hiperbólicos. O teorema que será apresentado a seguir relaxa esta hipótese, 
8.1 Comportamento da fronteira da região de estabilidade na presença de um ponto de equilíbrio sela-nó do tipo zero

e garante a persistência dos pontos de equilíbrio hiperbólicos na fronteira mesmo com a presença de um ponto de equilíbrio não hiperbólico sela-nó do tipo zero na fronteira da região de estabilidade. Para demonstrar o teorema precisaremos do seguinte resultado.

Lema 8.1.2. (SHUB, 1987) Sejam $z_{\lambda_{*}}$ e $w_{\lambda_{*}}$ pontos de equilíbrio hiperbólicos do sistema (7.1) para $\lambda=\lambda_{*}$. Se $W_{\lambda_{*}}^{u}\left(z_{\lambda_{*}}\right) \cap W_{\lambda_{*}}^{s}\left(w_{\lambda_{*}}\right) \neq \emptyset$, e $W_{\lambda_{*}}^{u}\left(z_{\lambda_{*}}\right)$ e $W_{\lambda_{*}}^{s}\left(w_{\lambda_{*}}\right)$ satisfazem a condição de transversalidade, então existe $\delta>0$ tal que $W_{\lambda}^{u}\left(z_{\lambda}\right) \cap W_{\lambda}^{s}\left(w_{\lambda}\right) \neq \emptyset$ para todo $\lambda \in\left(\lambda_{*}-\delta, \lambda_{*}+\delta\right)$ onde $z_{\lambda}$ e $w_{\lambda}$ são os pontos de equilíbrio perturbados.

Com o Lema 8.1.2, podemos demonstrar o teorema a seguir, que garante a persistência de pontos de equilíbrio hiperbólicos na fronteira da região de estabilidade mesmo na presença de um ponto de equilíbrio não hiperbólico sela-nó do tipo zero na fronteira.

Teorema 8.1.1. (Persistência de pontos de equilibrio hiperbólicos na fronteira da região de estabilidade) Seja $x_{\lambda_{0}}$ um ponto de equilíbrio sela-nó do tipo zero na fronteira da região de estabilidade $\partial A_{\lambda_{0}}\left(x_{\lambda_{0}}^{s}\right)$ de um ponto de equilíbrio hiperbólico assintoticamente estável $x_{\lambda_{0}}^{s}$ de (7.1) para $\lambda=\lambda_{0}$. Admita que as suposições $\left(A 1^{\prime}\right),(A 2),(A 3)$ e (A4) sejam satisfeitas para $\lambda=\lambda_{0}$. Se $x_{\lambda_{0}}^{i}, i=1, \ldots, k$ são os pontos de equilibrio hiperbólicos em $\partial A_{\lambda_{0}}\left(x_{\lambda_{0}}^{s}\right)$, então existe $\varepsilon>0$ tal que o ponto de equilíbrio perturbado $x_{\lambda}^{i} \in \partial A_{\lambda}\left(x_{\lambda}^{s}\right)$, para todo $i=1, \ldots$, k e $\lambda \in\left(\lambda_{0}-\varepsilon, \lambda_{0}+\varepsilon\right)$.

Demonstração. O Teorema da Função Implícita 6.1.1 garante a persistência dos pontos de equilíbrio hiperbólicos $x_{\lambda_{0}}^{i}, i=1, \ldots, k$ e do ponto de equilíbrio hiperbólico assintoticamente estável $x_{\lambda_{0}}^{s}$, sob pequenas variações do parâmetro $\lambda$. Em outras palavras, existem vizinhanças $U_{i}$ e $V$ de $x_{\lambda_{0}}^{i}$ e $x_{\lambda_{0}}^{s}$, respectivamente, e $\varepsilon_{1}>0$ tal que existe um único ponto de equilíbrio hiperbólico $x_{\lambda}^{i}$ em $U_{i}$ e um único ponto de equilíbrio hiperbólico assintoticamente estável $x_{\lambda}^{s}$ em $V$ para todo $i=1, \ldots, k$ e $\lambda \in\left(\lambda_{0}-\varepsilon_{1}, \lambda_{0}+\varepsilon_{1}\right)$. Desde que $x_{\lambda_{0}}^{i} \in \partial A_{\lambda_{0}}\left(x_{\lambda_{0}}^{s}\right)$ e as suposições $\left(A 1^{\prime}\right),(A 2)-(A 4)$ são satisfeitas para $\lambda=\lambda_{0}$, o Teorema 5.5.3 garante que $W_{\lambda_{0}}^{u}\left(x_{\lambda_{0}}^{i}\right) \cap A_{\lambda_{0}}\left(x_{\lambda_{0}}^{s}\right) \neq \emptyset$ para todo $i=1, \ldots, k$. Além disso, esta interseção é transversal. Por outro lado, como $A_{\lambda_{0}}\left(x_{\lambda_{0}}^{s}\right)=W_{\lambda_{0}}^{s}\left(x_{\lambda_{0}}^{s}\right)$, temos que $W_{\lambda_{0}}^{u}\left(x_{\lambda_{0}}^{i}\right) \cap$ $W_{\lambda_{0}}^{s}\left(x_{\lambda_{0}}^{s}\right) \neq \emptyset$ para todo $i=1, \ldots, k$. Logo, pelo Lema 8.1.2, podemos afirmar que existe $\varepsilon_{2}>0$ tal que $W_{\lambda}^{u}\left(x_{\lambda}^{i}\right) \cap W_{\lambda}^{s}\left(x_{\lambda}^{s}\right) \neq \emptyset$ para todo $i=1, \ldots, k$ e $\lambda \in\left(\lambda_{0}-\varepsilon_{2}, \lambda_{0}+\varepsilon_{2}\right)$, ou seja, $W_{\lambda}^{u}\left(x_{\lambda}^{i}\right) \cap A_{\lambda}\left(x_{\lambda}^{s}\right) \neq \emptyset$ para todo $i=1, \ldots, k$ e $\lambda \in\left(\lambda_{0}-\varepsilon_{2}, \lambda_{0}+\varepsilon_{2}\right)$. Portanto, o Teorema 4.1.1 garante que $x_{\lambda}^{i} \in \partial A_{\lambda}\left(x_{\lambda}^{s}\right)$ para todo $i=1, \ldots, k$ e $\lambda \in\left(\lambda_{0}-\varepsilon, \lambda_{0}+\varepsilon\right)$ com $\varepsilon=\min \left\{\varepsilon_{1}, \varepsilon_{2}\right\}$. Com isso, o teorema está provado.

Nos próximos resultados, estaremos interessados em estudar o comportamento da fronteira da região de estabilidade para pequenas variações do parâmetro $\lambda$ próximo a um parâmetro de bifurcação sela-nó do tipo zero $\lambda_{0}$. Neste estudo, será necessário controlar o aparecimento de novos pontos de equilíbrio quando parâmeros são variados, já que esse fenômeno pode ocorrer conforme foi visto no Exemplo 6.1.1. Sendo assim, admitiremos como hipótese nos próximos resultados a condição que para um parâmetro 
de bifurcação sela-nó do tipo zero $\lambda_{0}$, os pontos de equilíbrio do sistema $\dot{x}=f\left(x, \lambda_{0}\right)$ são todos hiperbólicos, exceto o ponto de equilíbrio não hiperbólico sela-nó do tipo zero $x_{\lambda_{0}}$. Além disso, para pequenas variações do parâmetro $\lambda$ próximo a $\lambda_{0}$, os pontos de equilíbrio do sistema perturbado $\dot{x}=f(x, \lambda)$ são exclusivamente os pontos de equilíbrio perturbados do sistema original $\dot{x}=f\left(x, \lambda_{0}\right)$.

O teorema a seguir estuda o comportamento da fronteira da região de estabilidade na vizinhança de um ponto de equilíbrio sela-nó do tipo zero.

\section{Teorema 8.1.2. (Comportamento da fronteira da região de estabilidade na vizinhança} de um ponto de equilíbrio sela-nó do tipo zero): Seja $\left(x_{\lambda_{0}}, \lambda_{0}\right)$ um ponto de bifurcação sela-nó do tipo zero de (7.1). Suponha que o ponto de equilíbrio sela-nó do tipo zero $x_{\lambda_{0}}$ pertença à fronteira da região de estabilidade $\partial A_{\lambda_{0}}\left(x_{\lambda_{0}}^{s}\right)$ de um ponto de equilíbrio hiperbólico assintoticamente estável $x_{\lambda_{0}}^{s}$ de (7.1) para $\lambda=\lambda_{0}$. Admita que a suposição (A3) seja satisfeita em um intervalo aberto I contendo o valor de bifurcação sela-nó do tipo zero $\lambda_{0}$ e a suposição (A4) é satisfeita para $\lambda=\lambda_{0}$. Além disso, assuma que o número de pontos de equilíbrio hiperbólicos do sistema (7.1) é finito e $x_{\lambda_{0}}$ é o único ponto de equilíbrio não hiperbólico para $\lambda=\lambda_{0}$. Suponha também, que para todo $\lambda \in I$, todos os pontos de equilíbrio do sistema perturbado $\dot{x}=f(x, \lambda)$ são pontos de equilíbrio perturbados originados do sistema $\dot{x}=f\left(x, \lambda_{0}\right)$. Então existe uma vizinhança $U$ de $x_{\lambda_{0}} e$ $\beta^{\prime} \geqslant \beta>0$ tal que:

(i) Existem somente dois pontos de equilíbrio hiperbólicos $y_{\lambda}^{s}$ e $y_{\lambda}^{u}$ em $U$ do tipo zero e tipo um, respectivamente para todo $\lambda \in\left(\lambda_{0}-\beta^{\prime}, \lambda_{0}\right)$ e não existe ponto de equilíbrio em $U$ para todo $\lambda \in\left(\lambda_{0}, \lambda_{0}+\beta^{\prime}\right)$. Além disso, a variedade estável do ponto de equilíbrio do tipo zero e a variedade instável do tipo um se interceptam ao longo de uma variedade unidimensional.

(ii) $y_{\lambda}^{u} \in \partial A_{\lambda}\left(x_{\lambda}^{s}\right) \cap \partial A_{\lambda}\left(y_{\lambda}^{s}\right)$ para todo $\lambda \in\left(\lambda_{0}-\beta, \lambda_{0}\right)$.

(iii) $U \subset A_{\lambda}\left(x_{\lambda}^{s}\right)$ para todo $\lambda \in\left(\lambda_{0}, \lambda_{0}+\beta\right)$.

Demonstração. (i) A existência da vizinhança $U$ e escalar $\beta^{\prime}$ com as propriedades desejadas é uma consequência direta do Teorema 7.2.2.

(ii) Primeiramente, mostraremos a existência de $\beta>0$ tal que $y_{\lambda}^{u} \in \partial A_{\lambda}\left(x_{\lambda}^{s}\right)$ para todo $\lambda \in\left(\lambda_{0}-\beta, \lambda_{0}\right)$. Sejam $x_{\lambda_{0}}^{i}, i=1 \ldots, k$, os pontos de equilíbrio hiperbólicos em $\partial A_{\lambda_{0}}\left(x_{\lambda_{0}}^{s}\right)$ e $x_{\lambda_{0}}^{j}, j=k+1, \ldots, m$, os pontos de equilíbrio hiperbólicos que não pertencem a $\partial A_{\lambda_{0}}\left(x_{\lambda_{0}}^{s}\right)$. Desde que o ponto de equilíbrio sela-nó do tipo zero $x_{\lambda_{0}} \in \partial A_{\lambda_{0}}\left(x_{\lambda_{0}}^{s}\right)$ e o número dos pontos de equilíbrio em $\partial A_{\lambda_{0}}\left(x_{\lambda_{0}}^{s}\right)$ é finito, então, pelo Lema 8.1.1, a vizinhança $U$ de $x_{\lambda_{0}}$ pode ser escolhida de tal modo que $W_{\lambda_{0}}^{s}\left(x_{\lambda_{0}}^{i}\right) \cap U=\emptyset$ para todo $i=1 \ldots, k$. Por outro lado, como $W_{\lambda_{0}}^{s}\left(x_{\lambda_{0}}^{j}\right) \cap U_{\lambda_{0}}^{-}=\emptyset$ e o Corolário 8.1.1 garante que $\left(U_{\lambda_{0}}^{+}-W_{\lambda_{0}}^{s}\left(x_{\lambda_{0}}\right)\right) \subset A_{\lambda_{0}}\left(x_{\lambda_{0}}^{s}\right)$, podemos afirmar que $W_{\lambda_{0}}^{s}\left(x_{\lambda_{0}}^{j}\right) \cap U=\emptyset$ para todo $j=k+1 \ldots, m$. Do fato que $x_{\lambda_{0}} \in \partial A_{\lambda_{0}}\left(x_{\lambda_{0}}^{s}\right)$, temos que $U \cap A_{\lambda_{0}}\left(x_{\lambda_{0}}^{s}\right) \neq \emptyset$, além disso, esta interseção é transversal. Desde que interseções vazias são transversais e interseções transversais persistem sob pequenas per- 
tubações, existe $\varepsilon_{2}>0$ tal que $U \cap A_{\lambda}\left(x_{\lambda}^{s}\right) \neq \emptyset, W_{\lambda}^{s}\left(x_{\lambda}^{i}\right) \cap U=\emptyset, W_{\lambda}^{s}\left(x_{\lambda}^{j}\right) \cap U=\emptyset$ para todo $i=1 \ldots, k, j=k+1 \ldots, m$ e $\lambda \in\left(\lambda_{0}-\varepsilon_{2}, \lambda_{0}+\varepsilon_{2}\right)$. Tomando $\beta=\min \left\{\varepsilon_{2}, \beta^{\prime}\right\}>0$, temos que $U \cap A_{\lambda}\left(x_{\lambda}^{s}\right) \neq \emptyset, W_{\lambda}^{s}\left(x_{\lambda}^{i}\right) \cap U=\emptyset \forall i=1, \ldots, k, W_{\lambda}^{s}\left(x_{\lambda}^{j}\right) \cap U=\emptyset \forall j=k+1, \ldots, m$ e existem dois pontos de equilíbrio hiperbólicos $y_{\lambda}^{s}$ e $y_{\lambda}^{u}$ em $U$ para todo $\lambda \in\left(\lambda_{0}-\beta, \lambda_{0}\right)$. Do fato que $U \cap A_{\lambda}\left(x_{\lambda}^{s}\right) \neq \emptyset$, a vizinhança $U$ é conexa e $U$ não está inteiramente contida em $A\left(x_{\lambda}^{s}\right)$, podemos afirmar que $U \cap \partial A_{\lambda}\left(x_{\lambda}^{s}\right) \neq \emptyset$ para todo $\lambda \in\left(\lambda_{0}-\beta, \lambda_{0}\right)$. Desde que a suposição $(A 3)$ seja satisfeita para todo $\lambda$ próximo a $\lambda_{0}$, podemos afirmar que a fronteira da região de estabilidade $\partial A_{\lambda}\left(x_{\lambda}^{s}\right)$ está contida na união das variedades estáveis dos pontos de equilíbrio em $\partial A_{\lambda}\left(x_{\lambda}^{s}\right)$. Como consequência, concluímos que $U$ intercepta a variedade estável de pelo menos um ponto de equilíbrio na fronteira $\partial A_{\lambda}\left(x_{\lambda}^{s}\right)$. Desde que, por hipótese os pontos de equilíbrio do sistema perturbado $\dot{x}=f(x, \lambda)$ são $x_{\lambda}^{i}, \forall i=1, \ldots, k, x_{\lambda}^{j} \forall j=k+1, \ldots, m, y_{\lambda}^{s}$ e $y_{\lambda}^{u}$ para todo $\lambda \in\left(\lambda_{0}-\beta, \lambda_{0}\right)$, além disso $W_{\lambda}^{s}\left(x_{\lambda}^{i}\right) \cap U=\emptyset \forall i=1, \ldots, k, W_{\lambda}^{s}\left(x_{\lambda}^{j}\right) \cap U=\emptyset \forall j=k+1, \ldots, m, y_{\lambda}^{s} \notin \partial A_{\lambda}\left(x_{\lambda}^{s}\right)$, pois $y_{\lambda}^{s}$ é assintoticamente estável e $W_{\lambda}^{u}\left(y_{\lambda}^{u}\right) \cap U \neq \emptyset$, necessariamente $y_{\lambda}^{u} \in \partial A_{\lambda}\left(x_{\lambda}^{s}\right)$ para todo $\lambda \in\left(\lambda_{0}-\beta, \lambda_{0}\right)$.

Mostraremos agora que $y_{\lambda}^{u} \in \partial A_{\lambda}\left(y_{\lambda}^{s}\right)$ para todo $\lambda \in\left(\lambda_{0}-\beta, \lambda_{0}\right)$. Do item $(i)$, temos que $W_{\lambda}^{s}\left(y_{\lambda}^{s}\right) \cap W_{\lambda}^{u}\left(y_{\lambda}^{u}\right) \neq \emptyset$, isto é, $A_{\lambda}\left(y_{\lambda}^{s}\right) \cap W_{\lambda}^{u}\left(y_{\lambda}^{u}\right) \neq \emptyset$, logo o Teorema 4.1.1 garante que $y_{\lambda}^{u} \in \partial A_{\lambda}\left(y_{\lambda}^{s}\right)$ para todo $\lambda \in\left(\lambda_{0}-\beta, \lambda_{0}\right)$.

(iii) Considerando o mesmo $\beta>0$ do item (ii), temos que os pontos de equilíbrio do sistema perturbado $\dot{x}=f(x, \lambda)$ são $x_{\lambda}^{i}, \forall i=1, \ldots, k, x_{\lambda}^{j} \forall j=k+1, \ldots, m$ para todo $\lambda \in$ $\left(\lambda_{0}, \lambda_{0}+\beta\right)$, além disso $U \cap A_{\lambda}\left(x_{\lambda}^{s}\right) \neq \emptyset, W_{\lambda}^{s}\left(x_{\lambda}^{i}\right) \cap U=\emptyset$ para todo $i=1, \ldots, k$ e $W_{\lambda}^{s}\left(x_{\lambda}^{j}\right) \cap$ $U=\emptyset$ para todo $j=k+1, \ldots, m$ e $\lambda \in\left(\lambda_{0}, \lambda_{0}+\beta\right)$. Em particular, de acordo com o item $(i)$, também não existe ponto de equilíbrio em $U$ para todo $\lambda \in\left(\lambda_{0}, \lambda_{0}+\beta\right)$. Do fato que $U \cap A_{\lambda}\left(x_{\lambda}^{s}\right) \neq \emptyset$, a fronteira da região de estabilidade $\partial A_{\lambda}\left(x_{\lambda}^{s}\right)$ está contida na união das variedades estáveis dos pontos de equilíbrio em $\partial A_{\lambda}\left(x_{\lambda}^{s}\right)$ e a vizinhança $U$ é conexa, podemos afirmar que $U \subset A_{\lambda}\left(x_{\lambda}^{s}\right)$ para todo $\lambda \in\left(\lambda_{0}, \lambda_{0}+\beta\right)$. Suponha, por contradição, que $U$ não esteja inteiramente contida em $A_{\lambda}\left(x_{\lambda}^{s}\right)$, então $U \cap \partial A_{\lambda}\left(x_{\lambda}^{s}\right) \neq \emptyset$ e consequentemente $U$ interceptaria a variedade estável de pelo menos um ponto de equilíbrio em $\partial A_{\lambda}\left(x_{\lambda}^{s}\right)$, levando-nos a um absurdo.

Do Teorema 8.1.2, para $\lambda<\lambda_{0}$ o ponto de equilíbrio hiperbólico do tipo um $y_{\lambda}^{u}$ em $U$ está na fronteira da região de estabilidade de $x_{\lambda}^{s}$. Quando $\lambda$ cresce, o ponto de equilíbrio estável $y_{\lambda}^{s}$ aproxima-se do ponto de equilíbrio hiperbólico do tipo um $y_{\lambda}^{u}$. Eles coalescem, dentro de $U$ em $\lambda=\lambda_{0}$ em um ponto de equilíbrio sela-nó do tipo zero $x_{\lambda_{0}}$. Em $\lambda=\lambda_{0}$, o ponto de equilíbrio sela-nó do tipo zero está na fronteira da região de estabilidade de $x_{\lambda_{0}}^{s}$. Quando $\lambda$ continua crescendo, o ponto de equilíbrio $x_{\lambda_{0}}$ desaparece e a vizinhança $U$ do ponto de equilíbrio sela-nó do tipo zero agora está contida na região de estabilidade de $x_{\lambda}^{s}$. O Teorema 8.1.2 mostra que a região de estabilidade e a fronteira da região de estabilidade sofrem mudanças drásticas em $\lambda=\lambda_{0}$. A fronteira da região de estabilidade 
8.1 Comportamento da fronteira da região de estabilidade na presença de um ponto de equilíbrio sela-nó do tipo zero

intercepta a vizinhança $U$ para $\lambda \leqslant \lambda_{0}$ enquanto $U$ está totalmente contida na região de estabilidade para $\lambda>\lambda_{0}$.

O próximo teorema é o passo inicial para entendermos o comportamento global da região de estabilidade e de sua fronteira sob variação do parâmetro nas vizinhanças de um parâmetro de bifurcação sela-nó do tipo zero.

Teorema 8.1.3. (A herança dos pontos de equilíbrio na fronteira da região de estabilidade): Seja $\left(x_{\lambda_{0}}, \lambda_{0}\right)$ um ponto de bifurcação sela-nó do tipo zero de (7.1). Suponha que o ponto de equilíbrio sela-nó do tipo zero $x_{\lambda_{0}}$ pertença à fronteira da região de estabilidade $\partial A_{\lambda_{0}}\left(x_{\lambda_{0}}^{s}\right)$ de um ponto de equilíbrio hiperbólico assintoticamente estável $x_{\lambda_{0}}^{s}$ de (7.1) para $\lambda=\lambda_{0}$. Admita que a suposição (A3) seja satisfeita em um intervalo aberto contendo o valor de bifurcação sela-nó do tipo zero $\lambda_{0}$ e as suposições (A2) e (A4) são satisfeitas para $\lambda=\lambda_{0}$. Além disso, assuma que o número de pontos de equilíbrio hiperbólicos do sistema (7.1) é finito e $x_{\lambda_{0}}$ é o único ponto de equilíbrio não hiperbólico para $\lambda=\lambda_{0}$. Suponha também, que para todo $\lambda \in I$, todos os pontos de equilíbrio do sistema perturbado $\dot{x}=f(x, \lambda)$ são pontos de equilíbrio perturbados originados do sistema $\dot{x}=f\left(x, \lambda_{0}\right)$. Se $x_{\lambda_{0}}^{*}$ é um ponto de equilíbrio hiperbólico em $\partial S^{*}\left(x_{\lambda_{0}}\right)$, então existe uma vizinhança $U$ de $x_{\lambda_{0}}, \beta^{\prime}>0, \eta>0$ e $\zeta>0$ tal que:

(i) Existem somente dois pontos de equilíbrio hiperbólicos $y_{\lambda}^{s}$ e $y_{\lambda}^{u}$ em $U$ do tipo zero e tipo um, respectivamente para todo $\lambda \in\left(\lambda_{0}-\beta^{\prime}, \lambda_{0}\right)$ e não existe ponto de equilíbrio em $U$ para todo $\lambda \in\left(\lambda_{0}, \lambda_{0}+\beta^{\prime}\right)$.

(ii) O ponto de equilíbrio perturbado $x_{\lambda}^{*} \in \partial A_{\lambda}\left(y_{\lambda}^{s}\right)$ para todo $\lambda \in\left(\lambda_{0}-\eta, \lambda_{0}\right)$.

(iii) O ponto de equilíbrio perturbado $x_{\lambda}^{*} \in \partial A_{\lambda}\left(x_{\lambda}^{s}\right)$ para todo $\lambda \in\left(\lambda_{0}, \lambda_{0}+\zeta\right)$.

Demonstração. (i) A existência da vizinhança $U$ e do escalar $\beta^{\prime}$ com as propriedades desejadas é uma consequência direta do Teorema 7.2.2.

(ii) Seja $x_{\lambda_{0}}^{*} \in \partial S_{\lambda_{0}}\left(x_{\lambda_{0}}\right)$. Sem perda de generalidade vamos admitir que a vizinhança $U$ é a mesma do Teorema 8.1.2. Do Teorema 5.6.4 podemos afirmar que $W_{\lambda_{0}}^{u}\left(x_{\lambda_{0}}^{*}\right) \cap S\left(x_{\lambda_{0}}\right) \neq$ $\emptyset$, em particular $W_{\lambda_{0}}^{u}\left(x_{\lambda_{0}}^{*}\right) \cap U_{\lambda_{0}}^{-} \neq \emptyset$. Seja $q \in W_{\lambda_{0}}^{u}\left(x_{\lambda_{0}}^{*}\right) \cap U_{\lambda_{0}}^{-}$, como $U_{\lambda_{0}}^{-}$é aberto, existe $r>$ 0 tal que $B(q ; r) \subset U_{\lambda_{0}}^{-}$. Além disso, dado $\alpha>0$ suficientemente pequeno, o número $r>0$ pode ser escolhido arbitrariamente pequeno tal que $B(q ; r) \subset U_{\lambda}^{-}$para todo $\lambda \in\left(\lambda_{0}-\right.$ $\left.\alpha, \lambda_{0}\right)$. Por outro lado, a variedade instável $W_{\lambda_{0}}^{u}\left(x_{\lambda_{0}}^{*}\right)$ intercepta $B(q ; r)$ transversalmente e como interseções transversais persistem a pequenas pertubações, existe $\varepsilon_{2}>0$ tal que $W_{\lambda}^{u}\left(x_{\lambda}^{*}\right) \cap B(q ; r) \neq \emptyset$ para todo $\lambda \in\left(\lambda_{0}-\varepsilon_{2}, \lambda_{0}+\varepsilon_{2}\right)$. Tomando $\eta=\min \left\{\alpha, \varepsilon_{2}, \beta^{\prime}\right\}$, temos que $W_{\lambda}^{u}\left(x_{\lambda}^{*}\right) \cap B(q ; r) \neq \emptyset$ e $B(q ; r) \subset U_{\lambda}^{-} \subset A_{\lambda}\left(y_{\lambda}^{s}\right)$ para todo $\lambda \in\left(\lambda_{0}-\eta, \lambda_{0}\right)$. Portanto, $W_{\lambda}^{u}\left(x_{\lambda}^{*}\right) \cap A_{\lambda}\left(y_{\lambda}^{s}\right) \neq \emptyset$ e pelo Teorema 4.1 .1 podemos afirmar que $x_{\lambda}^{*} \in \partial A_{\lambda}\left(y_{\lambda}^{s}\right)$ para todo $\lambda \in\left(\lambda_{0}-\eta, \lambda_{0}\right)$.

(iii) De acordo com o item $(i i)$, podemos afirmar que $W_{\lambda_{0}}^{u}\left(x_{\lambda_{0}}^{*}\right) \cap U \neq \emptyset$ e esta interseção é transversal. Como interseções transversais persistem a pequenas pertubações, existe 
8.1 Comportamento da fronteira da região de estabilidade na presença de um ponto de equilíbrio sela-nó

$\varepsilon_{3}>0$ tal que $W_{\lambda}^{u}\left(x_{\lambda}^{*}\right) \cap U \neq \emptyset$ para todo $\lambda \in\left(\lambda_{0}-\varepsilon_{3}, \lambda_{0}+\varepsilon_{3}\right)$. Tomando $\zeta=\min \left\{\varepsilon_{3}, \beta\right\}$ onde $\beta>0$ é definido no item (iii) do Teorema 8.1.2, temos que $W_{\lambda}^{u}\left(x_{\lambda}^{*}\right) \cap U \neq \emptyset \mathrm{e}$ $U \subset A_{\lambda}\left(x_{\lambda}^{s}\right)$ para todo $\lambda \in\left(\lambda_{0}, \lambda_{0}+\zeta\right)$. Portanto, $W_{\lambda}^{u}\left(x_{\lambda}^{*}\right) \cap A_{\lambda}\left(x_{\lambda}^{s}\right) \neq \emptyset$ e pelo Teorema 4.1.1 podemos afirmar que $x_{\lambda}^{*} \in \partial A_{\lambda}\left(x_{\lambda}^{s}\right)$ para todo $\lambda \in\left(\lambda_{0}, \lambda_{0}+\zeta\right)$.

O Teorema 8.1.3 mostra que a região de estabilidade de $x_{\lambda}^{s}$ "herda" toda a região de estabilidade do ponto de equilíbrio assintoticamente estável $y_{\lambda}^{s}$ que desaparece na bifurcação sela-nó do tipo zero em $\lambda=\lambda_{0}$. Tecnicamente, o Teorema 8.1.3 mostra que os pontos de equilíbrio na fronteira da região de estabilidade de $y_{\lambda}^{s}$ para $\lambda<\lambda_{0}$ são herdados pela fronteira da região de estabilidade de $x_{\lambda}^{s}$ quando $\lambda$ cresce e passa por $\lambda_{0}$.

As Figuras 8.2, 8.3 e 8.4 ilustram os Teoremas 8.1.2 e 8.1.3. A Figura 8.2 mostra a região de estabilidade do sistema (7.1) para $\lambda<\lambda_{0}$. Existem dois equilíbrios assintoticamente estáveis $x_{\lambda}^{s}$ e $y_{\lambda}^{s}$ nesta figura. O ponto de equilíbrio hiperbólico do tipo um $y_{\lambda}^{u}$ pertence à fronteira da região de estabilidade de ambos pontos de equilíbrio assintoticamente estáveis, enquanto $x_{\lambda}^{*}$ está na fronteira da região de estabilidade de $y_{\lambda}^{s}$. Quando $\lambda$ cresce, o sistema fica sujeito a uma bifurcação sela-nó do tipo zero em $\lambda=\lambda_{0}$. A Figura 8.3 ilustra a região de estabilidade do sistema (7.1) para $\lambda=\lambda_{0}$. Os pontos de equilíbrio $y_{\lambda}^{u}$ e $y_{\lambda}^{s}$ da Figura 8.2 coalescem em um único ponto de equilíbrio $x_{\lambda_{0}}$ na Figura 8.3. O ponto de equilíbrio $x_{\lambda_{0}}$ é um ponto de equilíbrio sela-nó do tipo zero que pertence à fronteira da região de estabilidade do ponto de equilíbrio hiperbólico assintoticamente estável $x_{\lambda_{0}}^{s}$. O ponto de equilíbrio $x_{\lambda}^{*}$ da Figura 8.2 persiste. O ponto de equilíbrio $x_{\lambda_{0}}^{*}$, que estava na fronteira da região de estabilidade do ponto de equilíbrio assintoticamente estável que desaparece na bifurcação, pertence agora à fronteira da região de estabilidade fraca do ponto de equilíbrio sela-nó do tipo zero $x_{\lambda_{0}}$. Quando $\lambda$ continua crescendo, o ponto de equilíbrio sela-nó do tipo zero e a região de estabilidade fraca desaparecem e o ponto de equilíbrio perturbado do tipo um $x_{\lambda}^{*}$, que pertencia à fronteira da região de estabilidade de $y_{\lambda}^{s}$ para $\lambda<\lambda_{0}$, pertence agora à fronteira da região de estabilidade de $x_{\lambda}^{s}$, ver Figura 8.4. Em outras palavras, a região de estabilidade do ponto de equilíbrio perturbado $x_{\lambda}^{s}$ "herda" a região de estabilidade do ponto de equilíbrio assintoticamente estável $y_{\lambda}^{s}$ que desapareceu na bifurcação sela-nó do tipo zero.

As demonstrações dos Teoremas 8.1.1, 8.1.2 e 8.1.3 também podem ser encontradas em (AMARAL; ALBERTO, 2010b).

Usando os Teoremas 5.5.4, 8.1.1, 8.1.2 e 8.1.3, obtemos o seguinte corolário que fornece uma caracterização da fronteira da região de estabilidade na vizinhança de um parâmetro de bifurcação sela-nó do tipo zero.

Corolário 8.1.2. (Caracterização da fronteira da região de estabilidade na vizinhança de um parâmetro de bifurcação sela-nó do tipo zero): Seja $\left(x_{\lambda_{0}}, \lambda_{0}\right)$ um ponto de bifurcação sela-nó do tipo zero de (7.1). Suponha que o ponto de equilíbrio sela-nó do tipo zero $x_{\lambda_{0}}$ pertença à fronteira da região de estabilidade $\partial A_{\lambda_{0}}\left(x_{\lambda_{0}}^{s}\right)$ de um ponto de 


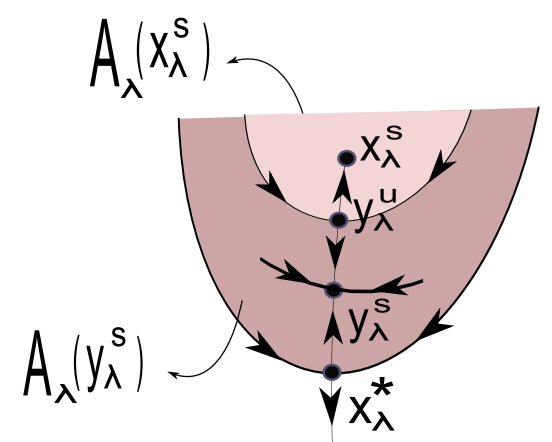

Figura 8.2: Região de estabilidade do sistema (7.1) para $\lambda<\lambda_{0}$. A área em roza mais escuro é a região de estabilidade do ponto de equilíbrio assintoticamente estável $y_{\lambda}^{s}$ enquanto a área em roza mais claro é a região de estabilidade de um ponto de equilíbrio assintoticamente estável $x_{\lambda}^{s}$.

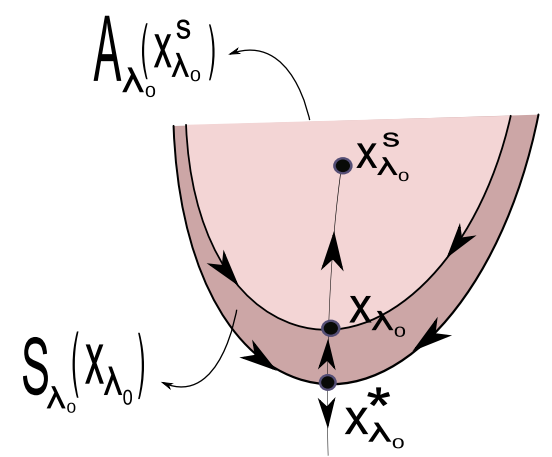

Figura 8.3: Região de estabilidade do sistema (7.1) para $\lambda=\lambda_{0}$. O ponto de equilíbrio assintoticamente estável $y_{\lambda}^{s}$ da Figura 8.2 coalesceu com o ponto de equilíbrio do tipo um $y_{\lambda}^{u}$ em um ponto de equilíbrio sela-nó do tipo zero $x_{\lambda_{0}}$. O ponto de equilíbrio sela-nó do tipo zero $x_{\lambda_{0}}$ está na fronteira da região de estabilidade de $x_{\lambda_{0}}^{s}$ e a área em roza mais escuro é a região de estabilidade fraca de $x_{\lambda_{0}}$.

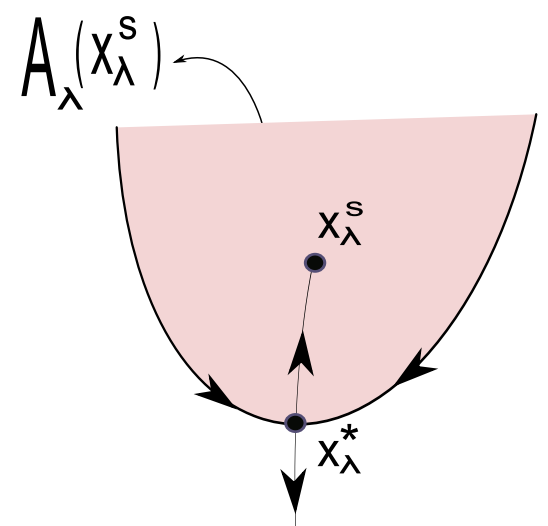

Figura 8.4: Região de estabilidade do sistema (7.1) para $\lambda>\lambda_{0}$. O ponto de equilíbrio hiperbólico do tipo um $x_{\lambda}^{*}$ que pertencia a fronteira da região de estabilidade de $y_{\lambda}^{s}$ para $\lambda<\lambda_{0}$ agora pertence a fronteira da região de estabilidade de $x_{\lambda}^{s}$. O ponto de equilíbrio $x_{\lambda}^{s}$ "herda" toda a região de estabilidade do ponto de equilíbrio $y_{\lambda}^{s}$, que desapareceu. 
equilíbrio hiperbólico assintoticamente estável $x_{\lambda_{0}}^{s}$ de (7.1) para $\lambda=\lambda_{0}$. Admita que as suposições $(A 2)$ e (A3) sejam satisfeitas em um intervalo aberto contendo o valor de bifurcação sela-nó do tipo zero $\lambda_{0}$ e a suposição (A4) está satisfeita para $\lambda=\lambda_{0}$. Além disso, assuma que o número de pontos de equilíbrio hiperbólicos do sistema (7.1) é finito e $x_{\lambda_{0}}$ é o único ponto de equilíbrio não hiperbólico para $\lambda=\lambda_{0}$. Suponha também, que para todo $\lambda \in I$, todos os pontos de equilíbrio do sistema perturbado $\dot{x}=f(x, \lambda)$ são pontos de equilíbrio perturbados originados do sistema $\dot{x}=f\left(x, \lambda_{0}\right)$. Se $x_{\lambda_{0}^{i}}^{*}$ são os pontos de equilíbrio em $\partial A_{\lambda_{0}}\left(x_{\lambda_{0}}^{s}\right), i=1, \ldots, k$ e $z_{\lambda_{0}^{l}}^{*}$ são os pontos de equilíbrios em $\partial S_{\lambda_{0}}^{*}\left(x_{\lambda_{0}}\right)$, $l=k+1, \ldots, m$, então:

(i) Para $\lambda=\lambda_{0}$ temos

$$
\partial A_{\lambda_{0}}\left(x_{\lambda_{0}}^{s}\right)=\bigcup_{i} W_{\lambda_{0}}^{s}\left(x_{\lambda_{0}^{i}}\right) \bigcup W_{\lambda_{0}}^{s}\left(x_{\lambda_{0}}\right)
$$

(ii) Existe $\varepsilon>0$ tal que, para todo $\lambda \in\left(\lambda_{0}-\varepsilon, \lambda_{0}\right)$,

$$
\partial A_{\lambda}\left(x_{\lambda}^{s}\right)=\bigcup_{i} W_{\lambda}^{s}\left(x_{\lambda^{i}}\right) \bigcup W_{\lambda}^{s}\left(y_{\lambda}^{u}\right)
$$

onde $x_{\lambda i}, i=1,2, \ldots, k$ são os pontos de equilíbrio hiperbólicos perturbados em $\partial A_{\lambda}\left(x_{\lambda}^{s}\right)$ e $y_{\lambda}^{u}$ é o ponto de equilíbrio hiperbólico do tipo um adicional, originado da bifurcação sela-nó do tipo zero, que também pertence a $\partial A_{\lambda}\left(x_{\lambda}^{s}\right)$.

(iii) Existe $\varepsilon>0$ tal que, para todo $\lambda \in\left(\lambda_{0}, \lambda_{0}+\varepsilon\right)$,

$$
\partial A_{\lambda}\left(x_{\lambda}^{s}\right)=\bigcup_{i} W_{\lambda}^{s}\left(x_{\lambda^{i}}\right) \bigcup_{l} W_{\lambda}^{s}\left(z_{\lambda^{l}}^{*}\right)
$$

onde $x_{\lambda^{i}}, i=1,2, \ldots, k$ e $z_{\lambda l}^{*}, l=k+1, \ldots, m$ são os pontos de equilíbrio hiperbólicos perturbados em $\partial A_{\lambda}\left(x_{\lambda}^{s}\right)$.

\subsection{Exemplos e Aplicações}

Exemplo 8.2.1. Considere o sistema de equações diferenciais

$$
\begin{aligned}
& \dot{x}=x^{2}+y^{2}-1 \\
& \dot{y}=x^{2}-y+\lambda
\end{aligned}
$$

onde $(x ; y) \in \mathbb{R}^{2}$ e $\lambda \in \mathbb{R}$.

Para $\lambda_{0}=-1$, vimos no Exemplo 5.1 que a fronteira da região de estabilidade $\partial A_{\lambda_{0}}(-1 ; 0)$ é formada pela união da variedade estável do ponto de equilíbrio hiperbólico $(1 ; 0)$ do tipo 1 com a variedade estável do ponto de equilíbrio sela-nó do tipo zero $(0 ;-1)$, ver Figura 8.6. Para $\lambda=-1.02$, o sistema (8.1) possui quatro pontos de equi- 
líbrio; são eles $x_{\lambda}^{s}=(-0,99 ;-0,02)$, um ponto de equilíbrio assintoticamente estável, $x_{\lambda}^{*}=(0,99 ;-0,02)$ um ponto de equilíbrio hiperbólico do tipo um, $y_{\lambda}^{u}=(-0,2 ;-0,97)$ um ponto de equilíbrio hiperbólico do tipo um e $y_{\lambda}^{s}=(0,2 ;-0,97)$ um ponto de equilíbrio hiperbólico do tipo zero. Os pontos de equilíbrios $y_{\lambda}^{u}$ e $y_{\lambda}^{s}$ são originados do ponto de equilíbrio sela-nó do tipo zero em uma bifurcação sela-nó do tipo zero. Além disso, $y_{\lambda}^{u} \in \partial A_{\lambda}(0,2 ;-0,97) \cap \partial A_{\lambda}(-0,99 ;-0,02)$, confirmando os resultados do Teorema 8.1.2, e $x_{\lambda}^{*}=(0,99 ;-0,02) \in \partial A_{\lambda}(-0,99 ;-0,02)$, confirmando o resultado do Teorema 8.1.1, ver Figura 8.5. Para $\lambda=-0,98$, o sistema (8.1) possui dois pontos de equilíbrio; são eles $x_{\lambda}^{s}=(-0,99 ; 0,01)$, um ponto de equilíbrio hiperbólico assintoticamente estável e $x_{\lambda}^{*}=(0,99 ; 0,01)$, um ponto de equilíbrio hiperbólico do tipo um, que pertence à fronteira da região de estabilidade $\partial A_{\lambda}(-0,99 ; 0,01)$ e confirma o resultado do Teorema 8.1.1, ver Figura 8.7.

Exemplo 8.2.2. Considere o sistema de equações diferenciais

$$
\begin{aligned}
& \dot{x}=x^{4}-1,25 x^{2}-y+0,25 \\
& \dot{y}=-y+\lambda
\end{aligned}
$$

onde $(x ; y) \in \mathbb{R}^{2}$ e $\lambda \in \mathbb{R}^{2}$.

Para $\lambda_{0}=0,25$, vimos no Exemplo 5.6.1 que a fronteira da região de estabilidade $\partial A_{\lambda_{0}}(-1,11 ; 0,25)$ é composta pela variedade estável do ponto de equilíbrio sela-nó do tipo zero $x_{\lambda_{0}}=(0 ; 0,25)$ e o ponto de equilíbrio hiperbólico do tipo um $x_{\lambda_{0}}^{*}=(1,11 ; 0,25)$ pertence a fronteira da região de estabilidade fraca de $(0 ; 0,25)$, ver Figura 8.9. Para $\lambda=0,2$, o sistema (8.2) possui quatro pontos de equilíbrio; são eles $x_{\lambda}^{s}=(-1,09 ; 0,2)$, um ponto de equilíbrio hiperbólico assintoticamente estável, $x_{\lambda}^{*}=(1,09 ; 0,2)$ um ponto de equilíbrio hiperbólico do tipo um, $y_{\lambda}^{u}=(-0,2 ; 0,2)$ um ponto de equilíbrio hiperbólico do tipo um e $y_{\lambda}^{s}=(0,2 ; 0,2)$ um ponto de equilíbrio hiperbólico do tipo zero. Os pontos de equilíbrio $y_{\lambda}^{u}$ e $y_{\lambda}^{s}$ são originados do ponto de equilíbrio sela-nó do tipo zero em uma bifurcação sela-nó do tipo zero. Além disso, $y_{\lambda}^{u} \in \partial A_{\lambda}(-1,09 ; 0,2) \cap \partial A_{\lambda}(0,2 ; 0,02)$, de acordo com o Teorem 8.1.2, e $x_{\lambda}^{*}=(1,09 ; 0,02) \in \partial A_{\lambda}(0,2 ; 0,02)$, confirmando os resultados do Teorema 8.1.3, ver Figura 8.8. Para $\lambda=0,3$, o sistema (8.2) possui dois pontos de equilíbrio; são els $x_{\lambda}^{s}=(-1,13 ; 0,3)$ um ponto de equilíbrio hiperbólico assintoticamente estável e $x_{\lambda}^{*}=(1,13 ; 0,3)$ um ponto de equilíbrio hiperbólico do tipo um que pertence a fronteira da região de estabilidade $\partial A_{\lambda}(-1,13 ; 0,3)$ de acordo com o Teorema 8.1.3, ver Figura 8.10.

Exemplo 8.2.3. Considere o sistema de equações diferenciais que modela uma Rede Neural Artificial de Hopfield :

$$
\dot{x_{i}}=\frac{1}{C_{i}}\left(\sum_{j=1}^{2} T_{i j} g_{j}\left(x_{j}\right)-\frac{1}{R_{i}} x_{i}+I_{i}\right)
$$


onde $i=1,2, x_{i} \in \mathbb{R}, g_{i}\left(x_{i}\right)=\frac{2}{\pi} \tan ^{-1}\left(\frac{\alpha \pi x_{i}}{2}\right)$. A constante $\alpha>0$ é a inclinação de $g_{i}\left(x_{i}\right)$ em $x_{i}=0$ enquanto $C_{i}>0, T_{i j}$ e $R_{i}$ são constantes físicas. As entradas $I_{i}$ da rede serão consideradas parâmetros do sistema.

Considerando $T_{11}=4,5, T_{12}=T_{21}=T_{22}=C_{1}=C_{2}=R_{1}=R_{2}=1, \alpha=0,3, I_{2}=0,02$ e variando o parâmetro $I_{1}$, uma bifurcação sela-nó do tipo zero ocorre no valor de bifurcação $I_{1}=0,418$. Mais precisamente, o sistema (8.3) possui, para $I_{1}=0,418$, dois pontos de equilíbrios; são eles $x_{\lambda_{0}}=(-1,44 ;-0,51)$, um ponto de equilíbrio sela-nó do tipo zero, e $x_{\lambda_{0}}^{s}=(3,69 ; 0,95)$, um ponto de equilíbrio hiperbólico assintoticamente estável. O ponto de equilíbrio sela-nó do tipo zero pertence à fronteira da região de estabilidade de $x_{\lambda_{0}}^{s}=(3,69 ; 0,95)$. A fronteira da região de estabilidade de $\partial A_{\lambda_{0}}(3,69 ; 0,95)$, é formada, de acordo com o Teorema 5.5.4, pela variedade estável do ponto de equilíbrio sela-nó do tipo zero $(-1,44 ;-0,51)$, ver Figura 8.13. Para $\lambda=0,4$, o sistema (8.3) possui três pontos de equilíbrio; são eles $x_{\lambda}^{s}=(3,66 ; 0,95)$, um ponto de equilíbrio hiperbólico assintoticamente estável, $y_{\lambda}^{u}=(-1,17 ;-0,42)$ um ponto de equilíbrio hiperbólico do tipo um e $y_{\lambda}^{s}=(-1,74 ;-0,59)$ um ponto de equilíbrio hiperbólico do tipo zero. Os pontos de equilíbrio $y_{\lambda}^{u}$ e $y_{\lambda}^{s}$ são originados do ponto de equilíbrio sela-nó em uma bifurcação selanó do tipo zero. Além disso, $y_{\lambda}^{u} \in \partial A_{\lambda}(3,66 ; 0,95) \cap \partial A_{\lambda}(-1,74 ;-0,59)$, de acordo com o Teorema 8.1.2, ver Figura 8.12. Para $\lambda=0,43$, o sistema (8.3) possui um único ponto de equilíbrio globalmente estável $x_{\lambda}^{s}=(3,71 ; 0,95)$, ver Figura 8.14. Este exemplo mostra que o relacionamento entre entradas e saídas da Rede Neural Artificial de Hopfield pode não ser única. Dependendo da história, a saída da rede pode ser diferente para a mesma entrada. A Figura 8.11 mostra uma diagrama de bifurcação. Este mostra a coordenada $x_{1}$ dos pontos de equilíbrio $x=\left(x_{1} ; x_{2}\right)$ como uma função do valor de entrada $I_{1}$. Para $I_{1}=0,4$ a saída da rede é $(-1,74 ;-0,59)$. Quando $I_{1}$ cresce, esse equilíbrio muda lentamente até o parâmetro de bifurcação $I_{1}=0,418$ ser atingido. Se $\lambda$ continua crescendo, então a saída da rede salta de $(-1,44 ;-0,51)$ para $(3,69 ; 0,95)$ como mostrado na Figura 8.11. Para $I_{1}=0,5$, por exemplo a saída da rede é $(3,82 ; 0,97)$. Se agora a entrada $I_{1}$ decresce para o valor inicial 0,4 , a saída será $(3,66 ; 0,95)$, que é diferente da saída inicial para o mesmo valor da entrada. Este comportamento pode ter consequências importantes quando a rede é usada por exemplo para reconhecimento de padrões. 


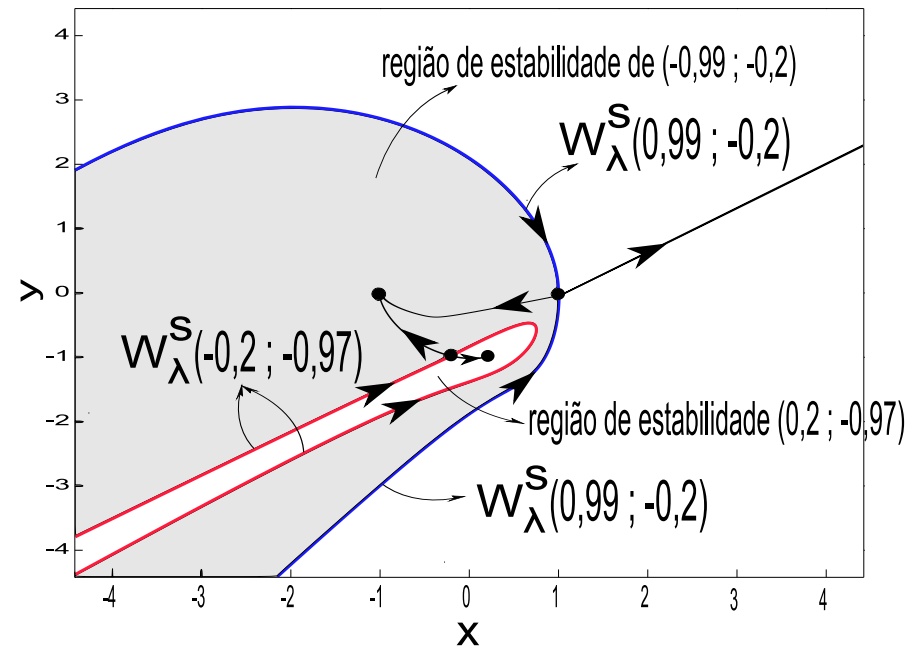

Figura 8.5: O retrato de fase do sistema (8.1) para $\lambda=-1,02$. A região de estabilidade do ponto de equilíbrio assintoticamente estável $(-0,99 ;-0,02)$ é representada pela área em cinza. A fronteira da região de estabilidade é constituída pela união da variedade estável do ponto de equilíbrio hiperbólico instável $(-0,2 ;-0,97)$, originado da bifurcação selanó do tipo zero, a curva em vermelho, com a variedade estável do ponto de equilíbrio hiperbólico $(0,99 ;-0,02)$, a curva em azul.

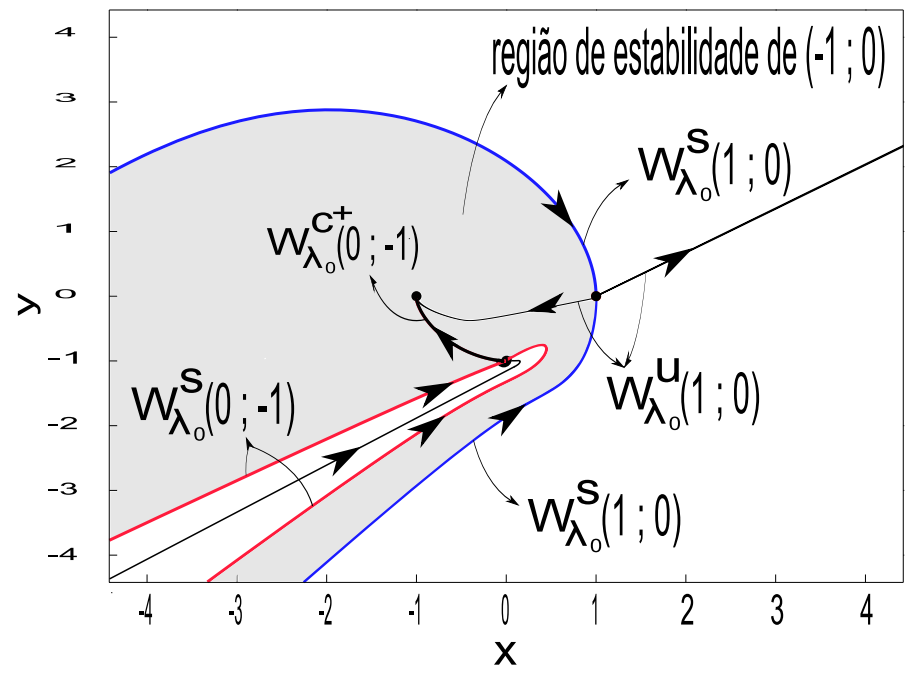

Figura 8.6: $\mathrm{O}$ retrato de fase do sistema (8.1) para $\lambda_{0}=-1$. A região de estabilidade do ponto de equilíbrio assintoticamente estável $(-1 ; 0)$ é representada pela área em cinza. A fronteira da região de estabilidade é constituída pela união da variedade estável do ponto de equilíbrio sela-nó do tipo zero $(0 ;-1)$, a curva em vermelho, com a variedade estável do ponto de equilíbrio hiperbólico $(1 ; 0)$, a curva em azul. 


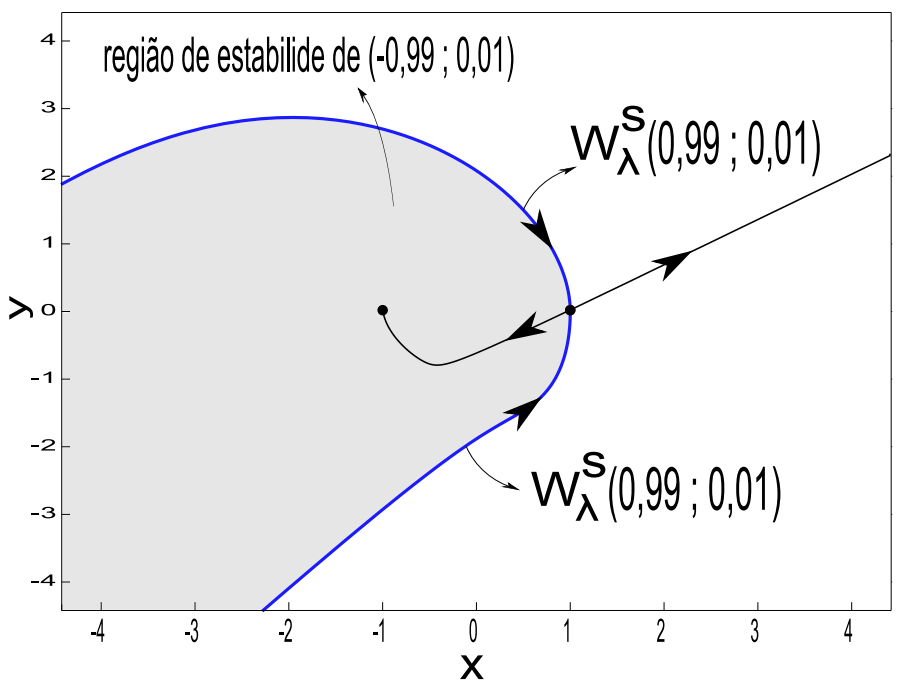

Figura 8.7: O retrato de fase do sistema (8.1) para $\lambda=-0,98$. A região de estabilidade do ponto de equilíbrio assintoticamente estável $(-0,99 ; 0,01)$ é representada pela área em cinza. A fronteira da região de estabilidade é constituída pela variedade estável do ponto de equilíbrio hiperbólico $(0,99 ; 0,01)$, a curva em azul.

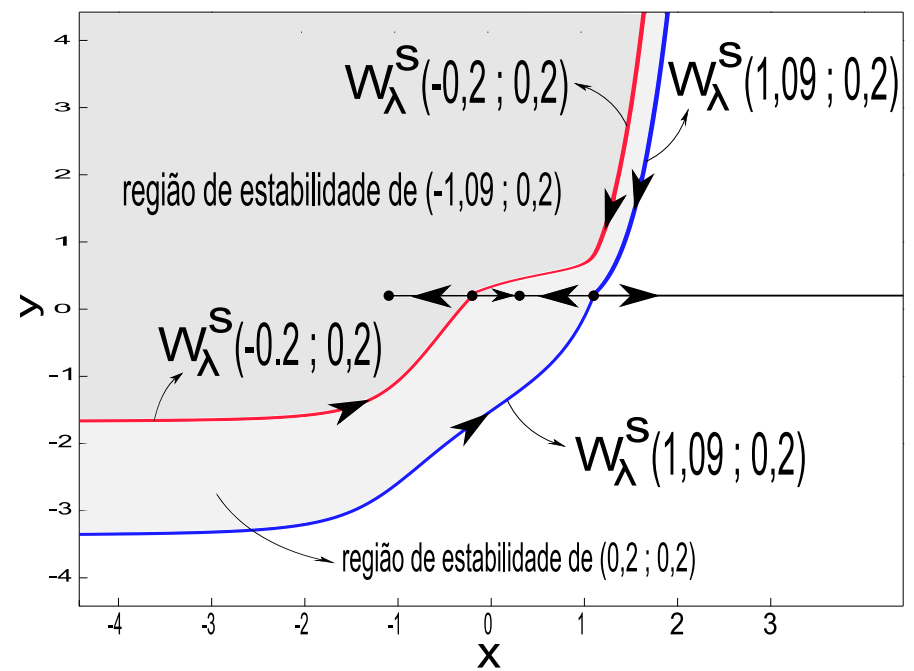

Figura 8.8: $\mathrm{O}$ retrato de fase do sistema (8.2) para $\lambda=0,2$. A região de estabilidade do ponto de equilíbrio assintoticamente estável $(-1,09 ; 0,2)$ é representada pela área em cinza escuro. A região de estabilidade do ponto de equilíbrio hiperbólico assintoticamente estável $(0,2 ; 0,2)$, originado da bifurcação sela-nó do tipo zero, é representada pela área em cinza claro. A fronteira da região de estabilidade $\partial A_{\lambda}(-1,09 ; 0,2)$ é constituída pela variedade estável do ponto de equilíbrio hiperbólico instável $(-0,2 ; 0,2)$, originado da bifurcação sela-nó do tipo zero, a curva em vermelho. A fronteira da região de estabilidade $\partial A_{\lambda}(0,2 ; 0,2)$ é constituída pela união da variedade estável do ponto de equilíbrio $(-0,2 ; 0,2)$, com a variedade estável do ponto de equilíbrio hiperbólico $(1,09 ; 0,2)$, a curva em azul. 


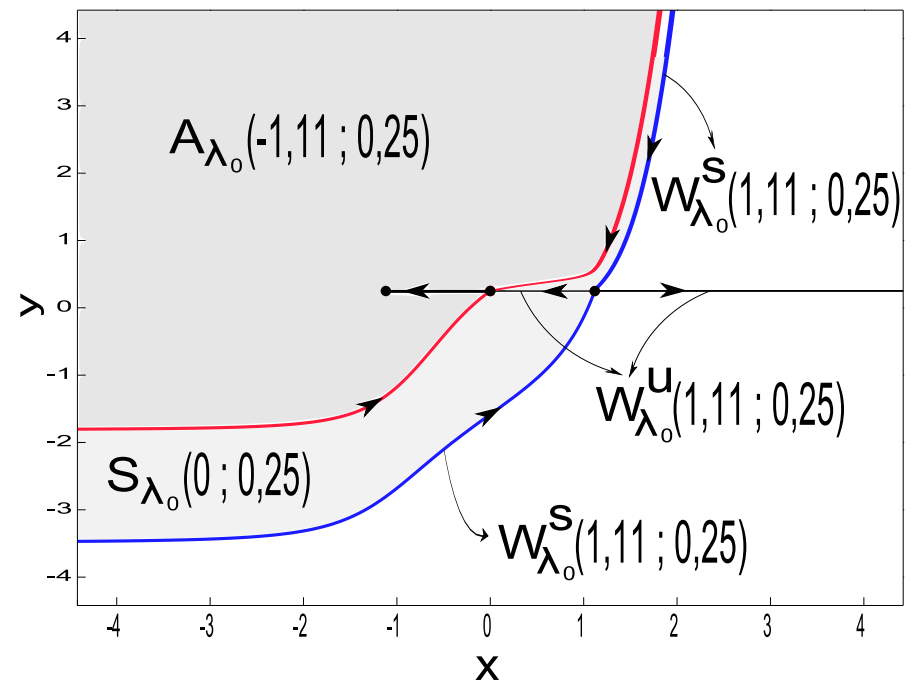

Figura 8.9: $O$ retrato de fase do sistema (8.2) para $\lambda_{0}=0,25$. A região de estabilidade do ponto de equilíbrio assintoticamente estável $(-1,11 ; 0,25)$ é representada pela área em cinza escuro. A região de estabilidade fraca de $(0 ; 0,25)$ é representada pela área em cinza claro. A fronteira da região de estabilidade $\partial A_{\lambda_{0}}(-1,11 ; 0,25)$ é constituída pela variedade estável do ponto de equilíbrio sela-nó do tipo zero $(0 ;, 0,25)$, a curva em vermelho. A fronteira da região de estabilidade fraca $\partial S_{\lambda_{0}}(0 ; 0,25)$ é constituída pela união da variedade estável do ponto de equilíbrio sela-nó do tipo zero, com a variedade estável do ponto de equilíbrio hiperbólico $(1,11 ; 0,25)$, a curva em azul.

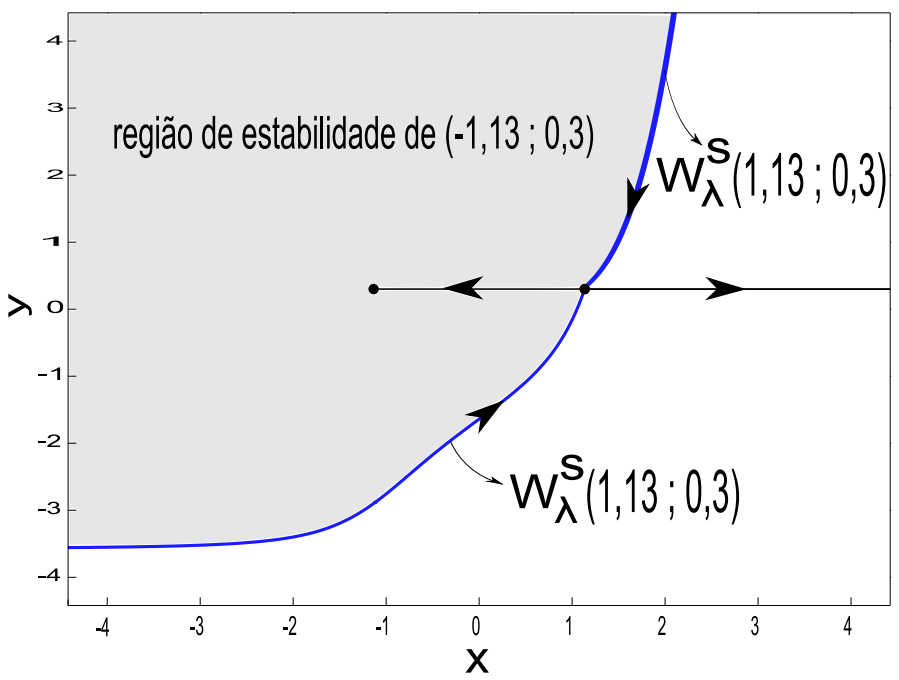

Figura 8.10: $\mathrm{O}$ retrato de fase do sistema (8.2) para $\lambda=0,3$. A região de estabilidade do ponto de equilíbrio hiperbólico assintoticamente estável $(-1,13 ; 0,3)$ é representada pela área em cinza escuro. A fronteira da região de estabilidade $\partial A_{\lambda}(-1,13 ; 0,2)$ é constituída pela variedade estável do ponto de equilíbrio hiperbólico instável $(1,13 ; 0,3)$, a curva em azul. 


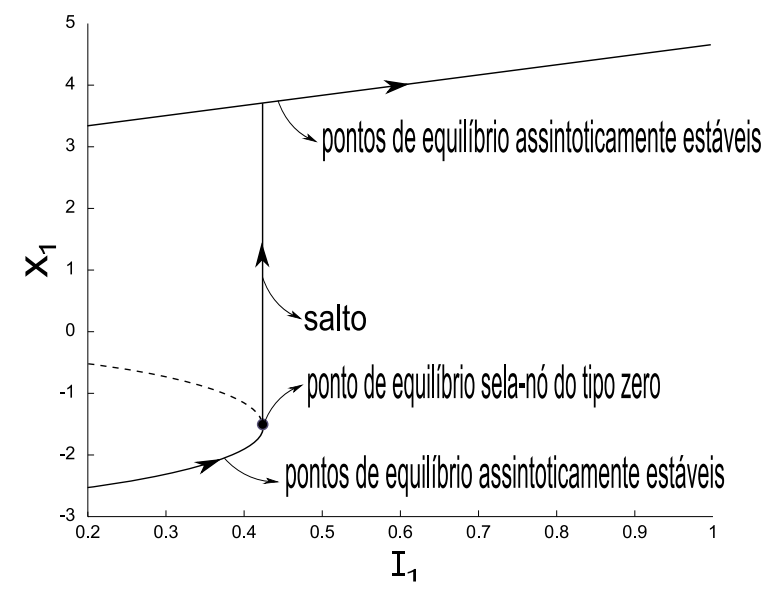

Figura 8.11: Diagrama de bifurcação do sistema (8.3). Este gráfico descreve a componente $x_{1}$ dos pontos de equilíbrio de (8.3) como uma função da entrada $I_{1}$. A linha tracejada corresponde a coordenada dos pontos de equilíbrio do tipo um enquanto a linha contínua indica os pontos de equilíbrio assintoticamente estáveis. Uma bifurcação sela-nó do tipo zero ocorre em $I_{1}=0,418$. Se o parâmetro $I_{1}$ cresce lentamente, então, para o valor de entrada 0,418 , a saída da rede salta rapidamente do ponto de equilíbrio sela-nó do tipo zero $(-1,44 ;-0,51)$ para o ponto de equilíbrio assintoticamente estável $(3,69 ; 0,95)$.

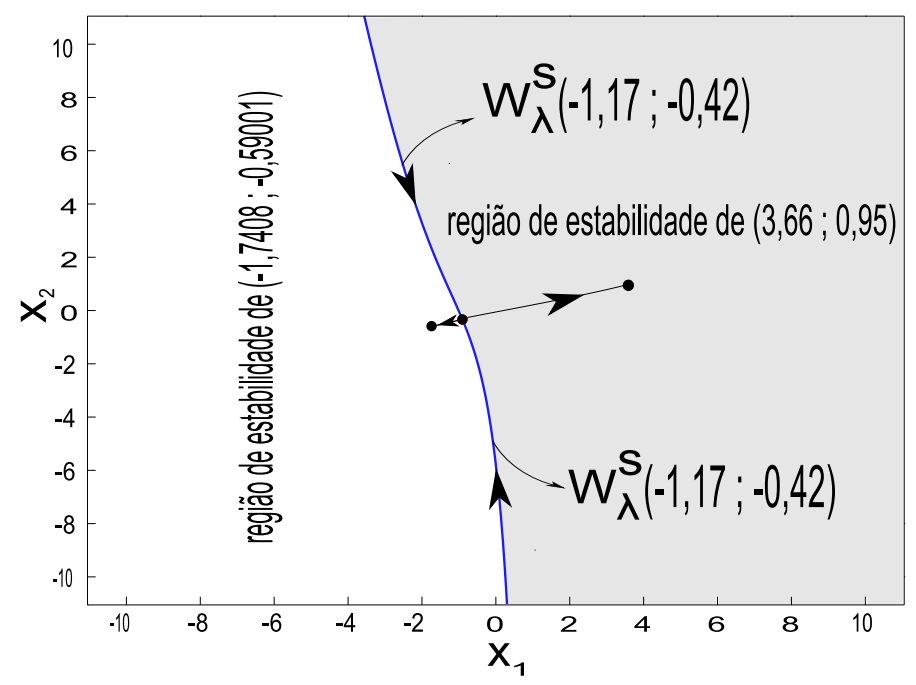

Figura 8.12: $O$ retrato de fase do sistema (8.3) para $I_{1}=0,4$. A região de estabilidade do ponto de equilíbrio assintoticamente estável $(3,66 ; 0,95)$ é representada pela área em cinza. A fronteira da região de estabilidade é constituída pela variedade estável do ponto de equilíbrio hiperbólico instável $(-1,17 ;-0,42)$, originado da bifurcação sela-nó do tipo zero, a curva em azul. 


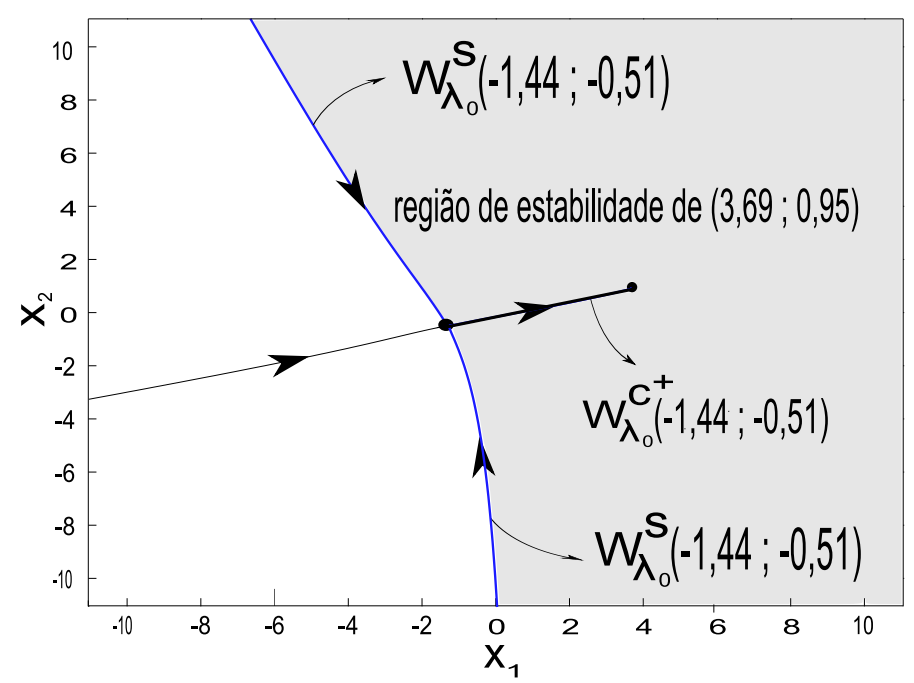

Figura 8.13: O retrato de fase do sistema (8.3) para $I_{1}=0,418$. A região de estabilidade do ponto de equilíbrio assintoticamente estável $(3,69 ; 0,95)$ é representada pela área em cinza. A fronteira da região de estabilidade é constituída pela variedade estável do ponto de equilíbrio sela-nó do tipo zero $(-1,44 ;-0,51)$, a curva em azul.

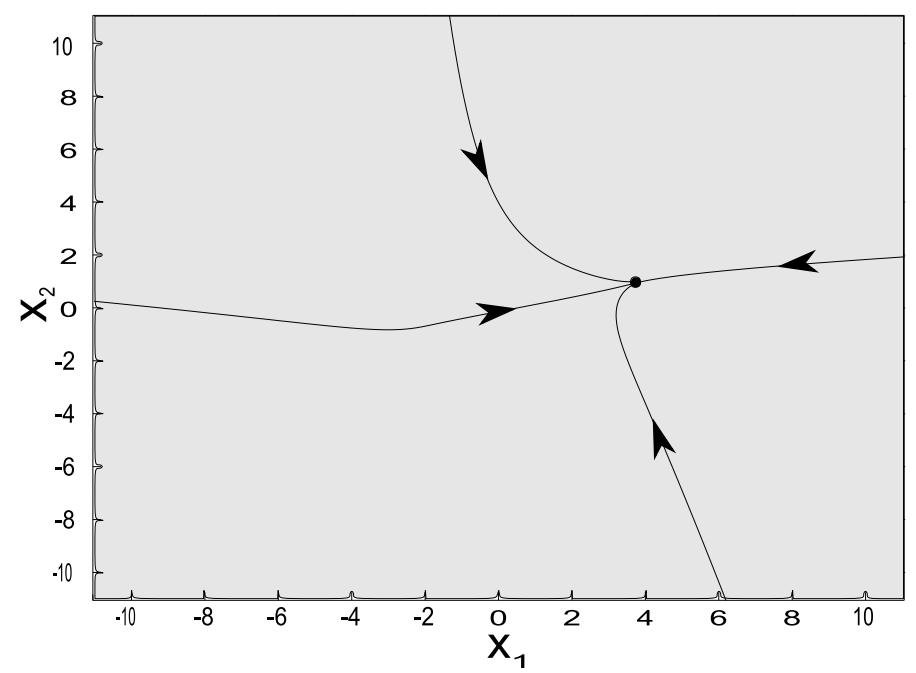

Figura 8.14: $\mathrm{O}$ retrato de fase do sistema (8.3) para $I_{1}=0,43$. O ponto de equilíbrio $(3,71 ; 0,95)$ é globalmente assintoticamente estável. 


\section{ESTIMATIVAS DA REGIÃO DE ESTABILIDADE COM BIFURCAÇÕES SELA-NÓ DO TIPO ZERO NA FRONTEIRA}

Neste capítulo, ofereceremos estimativas da região de estabilidade via conjunto de nível de uma dada função energia na presença de um ponto de equilíbrio sela-nó na fronteira e estudaremos o comportamento destas estimativas sob a influência das variações dos parâmetros, incluindo variações do parâmetro próximas a um parâmetro de bifurcação sela-nó do tipo zero. Na Seção 9.1, introduziremos o conceito de função energia existente literatura. Na Seção 9.2, faremos uma breve revisão dos resultados relacionados a estimativas da região de estabilidade propostas por Chiang et al. (1987).Na Seção 9.3, resultados que permitem caracterizar a fronteira da região de estabilidade na presença de um ponto de equilíbrio sela-nó na fronteira para sistemas que admitem função energia são apresentados. Na Seção 9.4, uma estimativa da região de estabilidade na presença de um ponto de equilíbrio sela-nó do tipo zero na fronteira é apresentada. Na Seção 9.5, um algoritmo conceitual para obter estimativas da região de estabilidade perturbada via conjunto de nível de uma dada função energia na vizinhança de um parâmetro de bifurcação sela-nó do tipo zero é apresentado.

\subsection{Função energia}

O conceito de função energia foi proposto na literatura por Chiang et al. (1987). A existência de uma função energia possui implicações importantes a respeito dos conjuntos limites, além disso, elas podem ser utilizadas para se obter estimativas da região de estabilidade (CHIANG; WU; VARAIYA, 1987; CHIANG; HIRSCH; WU, 1988).

Considere $E:=\left\{x \in \mathbb{R}^{n}: f(x)=0\right\}$ o conjunto dos pontos de equilíbrio do sistema (2.1).

Definição 9.1.1. (Função Energia)(CHIANG; WU; VARAIYA, 1987) A função $V: \mathbb{R}^{n} \rightarrow$ $\mathbb{R}$, de classe $C^{1}$, é uma função energia de (2.1) se as seguintes condições forem satisfeitas:

(i) $\dot{V}(x) \leqslant 0$ para todo $x \in \mathbb{R}^{n}$.

(ii) se $x_{0} \notin E$, então o conjunto $\left\{t \in \mathbb{R}_{+}: \dot{V}\left(\varphi\left(t, x_{0}\right)\right)=0\right\}$ possui medida nula em $\mathbb{R}$. 
(iii) se $V\left(\varphi\left(t, x_{0}\right)\right)$ é limitada para $t \in \mathbb{R}_{+}$, então a trajetória $\varphi\left(t, x_{0}\right)$ também é limitada para $t \in \mathbb{R}_{+}$.

As condicões $(i)$ e $(i i)$ implicam que a energia do sistema é não crescente ao longo de qualquer trajetória nao trivial. A a condição (iii) garante a não existência de uma trajetória ilimitada cuja energia permaneça limitada ao longo da trajetória. Uma condição suficiente para que (iii) seja satisfeita é que a função escalar $V: \mathbb{R}^{n} \rightarrow \mathbb{R}$ seja uma função própria $^{1}$ ou crescente. Por outro lado, podem existir funções escalares $V: \mathbb{R}^{n} \rightarrow \mathbb{R}$ onde a condição ( $i i i)$ é satisfeita embora elas não sejam própria e nem crescente, por exemplo as funções energias propostas nos Exemplos 9.2.1, 11.2.1 e 11.2.2.

A função energia assim como a função de Lyapunov são funções escalares auxiliares a partir das quais deseja-se obter informações a respeito da dinâmica do sistema não linear. O ojetivo da função de Lyapunov é o estudo de estabilidade(local) de um certo ponto de equilíbrio. Sendo assim, a função de Lyapunov basta ser definida positiva e sua derivada definida negativa numa vizinhança do ponto de equilíbrio em estudo. A função energia, por sua vez, tem o objetivo de extrair informações globais a respeito do comportamento dinâmico do sistema (2.1) como, por exemplo, informações a respeito dos conjuntos limites e da região de estabilidade de um ponto de equilíbrio assintoticamente estável. Sendo assim, a função energia deverá satisfazer as condições da Definição 9.1.1 para todo o espaço $\mathbb{R}^{n}$.

Na Definição 9.1.1 dada por Chiang et al. (1987), mas especificamente no item (ii), o conjunto $\left\{t \in \mathbb{R}_{+}: \dot{V}\left(\varphi\left(t, x_{0}\right)\right)=0\right\}$ tem que possuir medida nula em $\mathbb{R}$, se $x_{0} \notin E$. Um outro conceito de função energia tem sido proposto na literatura (SILVA et al., 2009) e exige ao invés da condição (ii) da Definição 9.1.1, que o conjunto $\left\{t \in \mathbb{R}_{+}: \dot{V}\left(\varphi\left(t, x_{0}\right)\right)<\right.$ $0\}$ não possua medida finita em $\mathbb{R}$, se $x_{0} \notin E$. Esta nova condição proposta no trabalho de Silva et al. (2009) é mais geral e contém o item (ii) da Definição 9.1.1 como um caso particular, ou seja, a exigência de que o conjunto $\left\{t \in \mathbb{R}_{+}: \dot{V}\left(\varphi\left(t, x_{0}\right)\right)=0\right\}$ possua medida nula em $\mathbb{R}$, se $x_{0} \notin E$, é uma condição suficiente para que o conjunto $\left\{t \in \mathbb{R}_{+}\right.$: $\left.\dot{V}\left(\varphi\left(t, x_{0}\right)\right)<0\right\}$ não possua medida finita em $\mathbb{R}$, se $x_{0} \notin E$. Nesta tese, vamos trabalhar com o conceito de função energia de acordo com a Definição 9.1.1.

\subsection{Estimativas da região de estabilidade}

Nesta seção, para obter estimativas da região de estabilidade, via conjunto de nível de uma dada função energia, serão apresentados resultados que exploram a caracterização da fronteira da região de estabilidade. Iniciaremos a seção apresentando um resultado que caracteriza a fronteira da região de estabilidade de sistemas dinâmicos autônomos não lineares que admitem uma função energia.

\footnotetext{
${ }^{1}$ Uma função contínua $V: \mathbb{R}^{n} \rightarrow \mathbb{R}$ é uma função própria se $\|x\| \rightarrow+\infty$ então $|V(x)| \rightarrow+\infty$
} 
Teorema 9.2.1. (Caracterização da fronteira da região de estabilidade)(CHIANG; $W U$; VARAIYA, 1987) Seja $x_{s}$ um ponto de equilíbrio assintoticamente estável de (2.1) e A(x $x_{S}$ sua região de estabilidade. Se existe uma função energia para o sistema (2.1) e a suposição (A1) está satisfeita, então:

$$
\partial A\left(x^{s}\right) \subseteq \bigcup_{i} W^{s}\left(x^{i}\right)
$$

onde $x^{i}, i=1,2, \ldots$ são os pontos de equilíbrio em $\partial A\left(x^{s}\right)$. Se, adicionalmente a suposição (A2) está satisfeita, então

$$
\partial A\left(x^{s}\right)=\bigcup_{i} W^{s}\left(x^{i}\right)
$$

Explorando a caracterização da fronteira da região de estabilidade do Teorema 9.2.1, o próximo teorema estuda a localização do mínimo da função energia na fronteira da região de estabilidade. Ele mostra que o mínimo da função energia é atingido em um ponto de equilíbrio.

Teorema 9.2.2. (Função energia e pontos de equilíbrio)(CHIANG; WU; VARAIYA, 1987) Considere o sistema dinâmico não linear (2.1), que admite uma função energia $V$. Seja $x_{s}$ um ponto de equilíbrio assintoticamente estável de (2.1) e $A\left(x_{s}\right)$ sua região de estabilidade. Suponha que (A1) esteja satisfeita. Então, na fronteira da região de estabilidade $\partial A\left(x_{s}\right)$, o ponto em que função energia $V$ assume o valor mínimo deve ser um ponto de equilíbrio.

O ponto de mínimo da função energia na fronteira da região de estabilidade pode não ser único, mas como a propriedade que todos os pontos de equilíbrio do sistema (2.1) têm valores da função energia distintos é genérica (CHIANG; THORP, 1989), podemos afirmar que genericamente o ponto de mínimo da função energia na fronteira da região de estabilidade é único, em outras palavras, quase sempre a unicidade do ponto de mínimo é garantida.

A importância prática e computacional deste resultado está no fato de podermos obter estimativas da região de estabilidade calculando o valor da energia apenas nos pontos de equilíbrio, os quais por sua vez podem ser numericamente calculados.

Explorando o fato de que o mínimo da função energia é atingido em um ponto de equilíbrio, o próximo teorema oferece entre outras coisas, um algoritmo para obter uma estimativa da região de estabilidade via conjunto de nível de uma dada função energia.

Teorema 9.2.3. (Estimativa da região de estabilidade via função energia)(CHIANG; WU; VARAIYA, 1987) Considere o sistema dinâmico não linear (2.1), que admite uma função energia $V$. Seja $x_{s}$ um ponto de equilíbrio assintoticamente estável de (2.1) e A $\left(x_{s}\right)$ sua região de estabilidade. Suponha que (A1) esteja satisfeita. Se $L=\min _{x_{i} \in \partial A\left(x^{s}\right) \cap E} V\left(x_{i}\right)$, $i=1,2, \ldots, k$ então: 
(i) a componente conexa $D(L)$ do conjunto de nível $\left\{x \in \mathbb{R}^{n}: V(x)<L\right\}$ contendo o equilíbrio $x^{s}$ está contida na região de estabilidade $A\left(x^{s}\right)$.

(ii) a componente conexa $D(B)$ do conjunto de nível $\left\{x \in \mathbb{R}^{n}: V(x)<B\right\}$ contendo o equilíbrio $x^{s}$ tem interseção não vazia com o complementar da região de estabilidade $A^{c}\left(x^{s}\right)$ para qualquer número real $B>L$.

A parte (ii) do Teorema 9.2.3 afirma que a escolha $L=\min _{x_{i} \in \partial A\left(x_{s}\right) \cap E} V\left(x_{i}\right)$ é ótima no sentido de que $D(L)$ é o maior conjunto, na forma de conjunto de nível da função energia $V$, inteiramente contido na região de estabilidade $A\left(x_{s}\right)$. Na prática, o Teorema 9.2.3 assegura que calculando todos os pontos de equilíbrio na fronteira da região de estabilidade, podemos obter uma estimativa da região de estabilidade.

Exibiremos a seguir um exemplo que ilustra os resultados apresentados nesta seção.

Exemplo 9.2.1. Considere o sistema de equações diferenciais

$$
\begin{aligned}
& \dot{x}=-y \\
& \dot{y}=\sin (x)-y+0,2
\end{aligned}
$$

onde $(x, y) \in \mathbb{R}^{2}$.

A função $V(x ; y)=-\cos (x)+0,2 x+\frac{y^{2}}{2}$ é uma função energia para o sistema (9.1). O sistema (9.1) possui três pontos de equilíbrio; são eles $(-0,2 ; 0)$, um ponto de equilíbrio hiperbólico assintoticamente estável, $(-2,94 ; 0)$ e $(3,34 ; 0)$ pontos de equilíbrio hiperbólicos instáveis. Ambos equilíbrios $(-2,94 ; 0)$ e $(3,34 ; 0)$ pertencem à fronteira da região de estabilidade $\partial A(-0,2 ; 0)$, ver Figura 9.1. O ponto de mínimo da função energia na fronteira $\partial A(-0,2 ; 0)$ é o ponto de equilíbrio $(-2,94 ; 0)$, pois $V(-2,94 ; 0)=$ $0,39<1,64=V(3,34 ; 0)$. A componente conexa $D(0,391)$ do conjunto de nível $\{(x, y) \in$ $\left.\mathbb{R}^{2}: V(x ; y)<0,39\right\}$ contendo o equilíbrio $(-0,2 ; 0)$ está inteiramente contida na região de estabilidade $A(-0,2 ; 0)$, de acordo com o Teorema 9.2.3, ver Figura 9.1. Tomando $B=0,41>0,39$, a componente conexa $D(0,41)$ do conjunto de nível $\left\{(x, y) \in \mathbb{R}^{2}\right.$ : $V(x ; y)<0,41\}$ contendo o ponto de equilíbrio $(-0,2 ; 0)$ intercepta o complementar da região de estabilidade $A^{c}(-0,2 ; 0)$, de acordo com o item (ii) do Teorema 9.2.3, ver Figura 9.2 .

\subsection{Função energia e a caracterização da fronteira da região de es- tabilidade na presença de um ponto de equilíbrio sela-nó do tipo zero}

Apresentaremos nesta seção uma caracterização da fronteira da região de estabilidade na presença de um ponto de equilíbrio sela-nó do tipo zero para sistemas que admitem função energia. Esta caracterização é uma generalização da caracterização da região de 


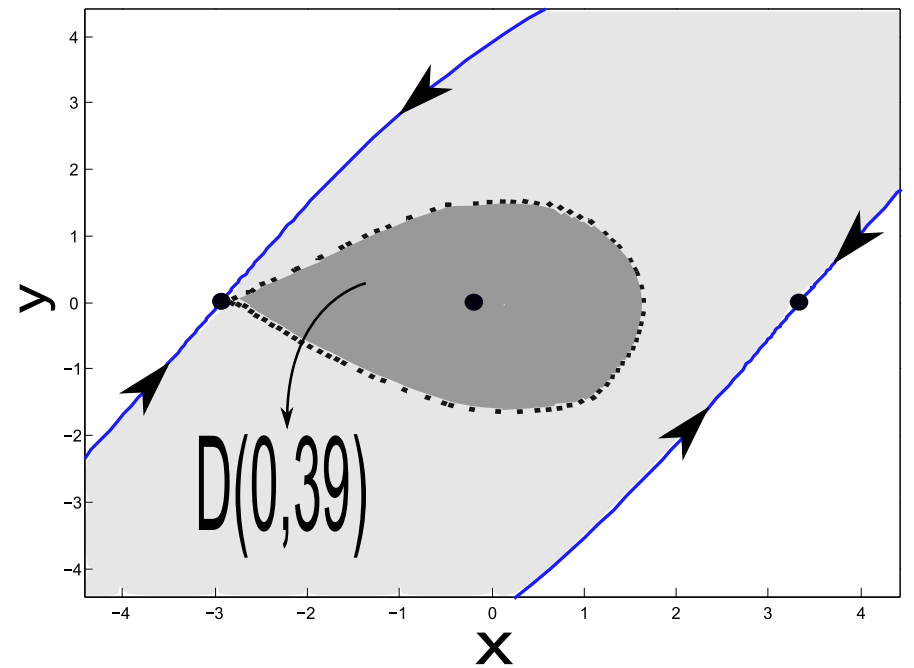

Figura 9.1: O retrato de fase do sistema (9.1). A região de estabilidade do ponto de equilíbrio assintoticamente estável $(-0,2 ; 0)$ é representada pela área em cinza claro. A componente conexa $D(0,39)$ do conjunto de nível $\left\{(x, y) \in \mathbb{R}^{2}: V(x ; y)<0,39\right\}$ contendo o equilíbrio $(-0,2 ; 0)$ representada pela área em cinza escuro é uma estimativa da região de estabilidade.

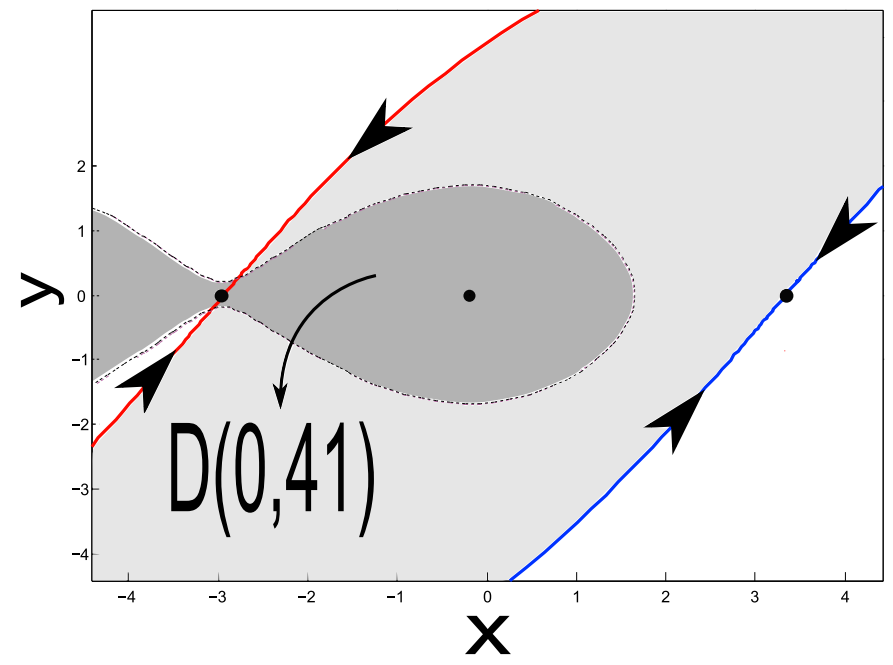

Figura 9.2: O retrato de fase do sistema (9.1). A região de estabilidade do ponto de equilíbrio assintoticamente estável $(-0,2 ; 0)$ é representada pela área em cinza claro. A componente conexa $D(0,41)$ do conjunto de nível $\left\{(x, y) \in \mathbb{R}^{2}: V(x ; y)<0,41\right\}$ contendo o equilíbrio $(-0,2 ; 0)$ representada pela área em cinza escuro intercepta o complementar da região de estabilidade $A^{c}(-0,2 ; 0)$.

estabilidade dada pelo Teorema 5.5.4. Iniciaremos a seção apresentando um resultado que estuda a implicação da existência de uma função energia em termos de conjuntos limites. A demonstração deste resultado pode ser encontrada em (SILVA et al., 2009).

O resultado a seguir estuda o comportamento do conjunto $\omega$-limite de uma solução $\varphi\left(t, x_{0}\right)$ do sistema (2.1) começando em uma condição inicial $x_{0}$, quando existe uma função energia $V$ associada ao sistema (2.1) satisfazendo a propriedade que $\left|V\left(\varphi\left(t, x_{0}\right)\right)\right|$ seja limitada para $t \geqslant 0$. 
9.3 Função energia e a caracterização da fronteira da região de estabilidade na presença de um ponto de equilíbrio sela-nó do tipo zero

Teorema 9.3.1. (SILVA et al., 2009) Se V é uma função energia do sistema (2.1), todos os pontos de equilíbrio em $E$ são isolados e $\varphi\left(t, x_{0}\right)$ é uma solução de (2.1) tal que $\left|V\left(\varphi\left(t, x_{0}\right)\right)\right|$ seja limitada para $t \geqslant 0$, então o conjunto $\omega\left(x_{0}\right)$ é composto por um único ponto de equilíbrio.

No Capítulo 5, oferecemos uma caracterização para a fronteira da região de estabilidade na presença de um ponto de equilíbrio sela-nó do tipo zero. Porém uma das hipóteses utilizadas nesta caracterização foi a suposição $(A 3)$ que não é satisfeita de maneira genérica na classe de sistemas dinâmicos da forma (2.1). O próximo teorema mostra que a existência de uma função energia é uma condição suficiente para garantir a satisfação da condição $(A 3)$ mesmo na presença de um ponto de equilíbrio não hiperbólico sela-nó do tipo zero na fronteira da região de estabilidade.

Teorema 9.3.2. (Condição Suficiente para (A3)) Seja $x^{s}$ um ponto de equilíbrio assintoticamente estável do sistema (2.1). Se existe uma função energia $V$ para o sistema (2.1) e a suposição $\left(A 1^{\prime}\right)$ está satisfeita, então (A3) é satisfeita, ou seja, todas as trajetórias na fronteira $\partial A\left(x^{s}\right)$ tendem para um ponto de equilíbrio quando $t \rightarrow+\infty$.

Demonstração. Seja $x_{0} \in \partial A\left(x^{s}\right)$ e $\varphi\left(t, x_{0}\right)$ uma trajetória passando por $x_{0}$, isto é, $\varphi\left(0, x_{0}\right)$ $=x_{0}$. Pela condição $(i)$ da Definição 9.1.1, temos que $V\left(\varphi\left(t, x_{0}\right)\right) \leqslant V\left(\varphi\left(0, x_{0}\right)\right)=V\left(x_{0}\right)$ para todo $t \geqslant 0$, ou seja, $V\left(\varphi\left(t, x_{0}\right)\right)$ é limitado superiormente por $V\left(x_{0}\right)$ para todo $t \geqslant 0$. Por outro lado, como $x_{0} \in \partial A\left(x^{s}\right)$, existe uma sequência de pontos $\left\{x_{i}\right\}, \operatorname{com} x_{i} \in A\left(x^{s}\right) \mathrm{e}$ $x_{i} \rightarrow x_{0}$ quando $i \rightarrow+\infty$. A continuidade de $V$ garante que $V\left(x_{i}\right) \rightarrow V\left(x_{0}\right)$ quando $i \rightarrow+\infty$. Além disso, a condição $(i)$ da Definição 9.1.1 garante que $V\left(x_{i}\right) \geqslant V\left(x^{s}\right)$ para todo $i \in \mathbb{N}$. Portanto, $V\left(x_{0}\right) \geqslant V\left(x^{s}\right)$ e a arbitrariedade da escolha de $x_{0}$ garante que $V$ é limitada inferiormente por $V\left(x^{s}\right)$ na fronteira $\partial A\left(x^{s}\right)$, isto é, $V(x) \geqslant V\left(x^{s}\right)$ para todo $x \in \partial A\left(x^{s}\right)$. Como a fronteira da região de estabilidade é invariante, $\varphi\left(t, x_{0}\right) \in \partial A\left(x^{s}\right)$ para todo $t \geqslant 0$, $\operatorname{logo} V\left(\varphi\left(t, x_{0}\right)\right) \geqslant V\left(x^{s}\right)$ para todo $t \geqslant 0$. Portanto, podemos afirmar que $\left|V\left(\varphi\left(t, x_{0}\right)\right)\right|$ é limitada para todo $t \geqslant 0$, já que $V\left(x^{s}\right) \leqslant V\left(\varphi\left(t, x_{0}\right)\right) \leqslant V\left(x_{0}\right)$ para todo $t \geqslant 0$. Como por hipótese a suposição $\left(A 1^{\prime}\right)$ é satisfeita, então os pontos de equilíbrio em $\partial A\left(x^{s}\right)$ são isolados, visto que ponto de equilíbrio hiperbólico e ponto de equilíbrio sela-nó do tipo zero são isolados. A invariância de $\partial A\left(x^{s}\right)$ e o Teorema 9.3.1 garantem que $\varphi\left(t, x_{0}\right) \rightarrow$ $E \cap \partial A\left(x^{s}\right)$ quando $t \rightarrow+\infty$. Isto é, toda trajetória na fronteira da região de estabilidade $\partial A\left(x^{S}\right)$ se aproxima de um ponto de equilíbrio em $\partial A\left(x^{S}\right)$ quando $t \rightarrow+\infty$.

Como uma consequência direta do Teorema 9.3.2 e Lema 5.4.5 obtemos o seguinte corolário.

Corolário 9.3.1. (Caracterização da Fonteira da Região de Estabilidade) Seja $A\left(x^{S}\right)$ a região de estabilidade de um ponto de equilíbrio assintoticamente estável $x^{s}$ de (2.1). Se existe uma função energia $V$ para o sistema (2.1) e a suposição $\left(A 1^{\prime}\right)$ está satisfeita, 
então:

$$
\partial A\left(x^{s}\right) \subseteq \bigcup_{i} W^{s}\left(x^{i}\right) \bigcup W^{s}(p)
$$

onde $x^{i}, i=1,2, \ldots$ são os pontos de equilíbrio hiperbólicos em $\partial A\left(x^{s}\right)$ e p é um ponto de equilíbrio sela-nó do tipo zero que também pertence à fronteira $\partial A\left(x^{S}\right)$.

Utilizando o Teorema 5.5.4 e o Corolário 9.3.1 apresentaremos, no próximo teorema, uma caracterização completa da fronteira da região de estabilidade na presença de um ponto de equilíbrio sela-nó do tipo zero.

Teorema 9.3.3. (Caracterização da fronteira da região de estabilidade): Seja $A\left(x^{S}\right)$ a região de estabilidade de um ponto de equilíbrio assintoticamente estável $x^{s}$ de (2.1), e suponha que p seja um ponto de equilíbrio sela-nó do tipo zero na fronteira da região de estabilidade $\partial A\left(x^{S}\right)$. Se existe uma função energia $V$ para o sistema (2.1) e as suposições $\left(A 1^{\prime}\right),(A 2)$ e (A4) são satisfeitas, então:

$$
\partial A\left(x^{s}\right)=\bigcup_{i} W^{s}\left(x^{i}\right) \bigcup W^{s}(p)
$$

onde $x^{i}, i=1,2, \ldots$ são os pontos de equilíbrio hiperbólicos em $\partial A\left(x^{s}\right)$ e p é um ponto de equilíbrio sela-nó do tipo zero que também pertence à fronteira $\partial A\left(x^{s}\right)$.

Demonstração. A existência da função energia garante, segundo o Corolário 9.3.2, que a suposição $(A 3)$ está satisfeita. Portanto, uma aplicação direta do Teorema 5.5.4 mostra que $\partial A\left(x^{s}\right)=\bigcup_{i} W^{s}\left(x^{i}\right) \bigcup W^{s}(p)$.

O Teorema 9.3.3 é uma generalização do Teorema 5.5.4. Ele mostra que a fronteira da região de estabilidade de um sistema que admite função energia é composta pela união das variedades estáveis de todos os pontos de equilíbrio na fronteira da região de estabilidade, incluindo a variedade estável do ponto de equilíbrio não hiperbólico sela-nó do tipo zero.

\subsection{Estimativas da região de estabilidade na presença de um ponto de equilíbrio sela-nó do tipo zero na fronteira}

Nesta seção, propomos uma estimativa da região de estabilidade, via conjuntos de nível de uma dada função energia, na presença de um ponto de equilíbrio sela-nó do tipo zero na fronteira. Iniciaremos a seção apresentando um resultado que generaliza o Teorema 9.2.2. Ele garante, que mesmo na presença de um ponto de equilíbrio não hiperbólico sela-nó do tipo zero na fronteira da região de estabilidade, que o ponto no qual a função energia assume o valor de mínimo na fronteira da região de estabilidade deve ser um ponto de equilíbrio. Na demonstração deste resultado exploramos o Corolário 9.3.1. 
Teorema 9.4.1. (Função energia e pontos de equilíbrio): Seja $A\left(x^{S}\right)$ a região de estabilidade de um ponto de equilíbrio assintoticamente estável $x^{s}$ de (2.1). Se existe uma função energia $V$ para o sistema (2.1) e a suposição $\left(A 1^{\prime}\right)$ é satisfeita, então o ponto no qual a função energia $V$ assume o valor de mínimo na fronteira da região de estabilidade deve ser um ponto de equilíbrio.

Demonstração. A demonstração deste teorema será feita por contradição. Sejam $x^{i}$, $i=1,2, .$. os pontos de equilíbrio hiperbólicos em $\partial A\left(x^{s}\right)$ e $p$ um possível ponto de equilíbrio sela-nó do tipo zero. Suponha que $q \in \partial A\left(x^{S}\right)$ é o ponto no qual a função energia $V$ assume o valor mínimo na fronteira $\partial A\left(x^{s}\right)$, mas $q \neq x^{i}$ para todo $i=1,2, \ldots$ e $q \neq p$. Pelo Corolário 9.3.1 podemos afirmar que $\lim _{t \rightarrow+\infty} \varphi(t, q)=x^{i}$ ou $\lim _{t \rightarrow+\infty} \varphi(t, q)=p$. A continuidade de $V$ garante que $\lim _{t \rightarrow+\infty} V(\varphi(t, q))=V\left(x^{i}\right)$ ou $\lim _{t \rightarrow+\infty} V(\varphi(t, q))=$ $V(p)$. Mas as condições $(i)-(i i)$ da Definição 9.1.1 implicam que dado $T>0$ arbitrariamente pequeno existe um tempo $t^{*} \in(0, T)$ tal que $V(q) \geqslant V\left(\varphi\left(t^{*}, q\right)\right)>V\left(x^{i}\right)$ ou $V(q) \geqslant V\left(\varphi\left(t^{*}, q\right)\right)>V(p)$, o que nos leva a uma contradição.

No Teorema 9.4.2, a suposição de que todos os pontos de equilíbrio na fronteira da região de estabilidade são hiperbólicos é fundamental para a validade do teorema. $\mathrm{O}$ Teorema 9.4.2 generaliza o Teorema 9.2.3 relaxando a suposição de hiperbolicidade $(A 1)$. Mais precisamente, ele fornece uma estimativa da região de estabilidade, via conjuntos de nível de uma dada função energia, na presença de um ponto de equilíbrio sela-nó do tipo zero na fronteira.

Teorema 9.4.2. (Estimativa da região de estabilidade por função energia): Seja $A\left(x^{S}\right)$ a região de estabilidade de um ponto de equilíbrio assintoticamente estável $x^{s}$ de (2.1). Seja p seja um ponto de equilíbrio sela-nó do tipo zero na fronteira da região de estabilidade $\partial A\left(x^{s}\right)$ e sejam $x_{i}, i=1, \ldots, k$ os pontos de equilíbrio hiperbólicos na fronteira da região de estabilidade $\partial A\left(x^{s}\right)$. Admita que exista uma função energia $V$ para o sistema (2.1) e a suposição $\left(A 1^{\prime}\right)$ esteja satisfeita. Se $L=\min _{x \in\left\{p, x^{i}\right\}} V(x), i=1, \ldots, k$ então:

i) a componente conexa $D(L)$ do conjunto de nível $\left\{x \in \mathbb{R}^{n}: V(x)<L\right\}$ contendo o equilíbrio $x^{s}$ está contida na região de estabilidade $A\left(x^{s}\right)$.

ii) a componente conexa $D(B)$ do conjunto de nível $\left\{x \in \mathbb{R}^{n}: V(x)<B\right\}$ contendo o equilíbrio $x^{s}$ tem interseção não vazia com o complementar da região de estabilidade $A^{c}\left(x^{s}\right)$ para qualquer número $B>L$.

Demonstração. A prova deste teorema será feita por contradição. Suponha que $D(L)$ não está contida em $A\left(x^{s}\right)$, isto é, $D(L) \cap A^{c}\left(x^{s}\right) \neq \emptyset$. Como $x^{s} \in D(L) \cap A\left(x^{s}\right)$, então $D(L) \cap A\left(x^{s}\right) \neq \emptyset$. A conexidade de $D(L)$ garante que $D(L) \cap \partial A\left(x^{s}\right) \neq \emptyset$. Seja $q \in$ $D(L) \cap \partial A\left(x^{s}\right)$. Então, $V(q)<L$ e $q \in \partial A\left(x^{s}\right)$. Mas $L$ é o valor mínimo de $V$ na fronteira da região de estabilidade $\partial A\left(x^{s}\right)$, o que nos leva a uma contradição.

(ii) Seja $B>L$. Então existem pontos na fronteira da região de estabilidade $\partial A\left(x^{S}\right)$ que pertencem a $D(B)$ e portanto $D(B) \cap A^{c}\left(x^{S}\right) \neq \emptyset$. 
Na prática, o Teorema 9.4.2 assegura que calculando todos os pontos de equilíbrio hiperbólicos e o ponto de equilíbrio não hiperbólico sela-nó do tipo zero na fronteira da região de estabilidade, podemos obter uma estimativa da região de estabilidade.

Exibiremos a seguir exemplos que ilustram os resultados apresentados nesta seção.

Exemplo 11.2.1 Considere o sistema de equações diferenciais

$$
\begin{aligned}
& \dot{x}=y+0,49 \\
& \dot{y}=\sin (3 x-1)-\cos (2 x-1,5)-y
\end{aligned}
$$

$\operatorname{com}(x, y) \in \mathbb{R}^{2}$.

O sistema (9.2) possui um ponto de equilíbrio hiperbólico assintoticamente estável $x^{s}=(3,43 ;-0,49)$ e três equilíbrios instáveis, são eles: $p=(1,68 ;-0,49)$ um ponto de equilíbrio sela-nó do tipo zero, $x^{1}=(4,48 ;-0,49)$ e $x^{2}=(0,44 ;-0,49)$ pontos de equilíbrio hiperbólicos do tipo um. Os três pontos de equilíbrio instáveis $p, x^{1}$ e $x^{2}$ pertencem à fronteira da região de estabilidade $\partial A(3,43 ;-0,49)$ que é composta pela união das variedades estáveis dos três equilíbrios, de acordo com o Teorema 9.3.3, ver Figura 9.3. A função $V(x ; y ;)=\frac{\cos (3 x-1)}{3}+\frac{\sin (2 x-1,5)}{2}+0,497(y-x)+\frac{y^{2}}{2}$ é uma função energia do sistema (9.2). A função energia $V(x ; y ;)$ é tal que $V(4,48 ;-0,49)=-1,55<-0,68=$ $V(1,68 ;-0,49) \quad<\quad V(0,44 ;-0,49)$ $=-0,31, \operatorname{logo}$ o ponto de equilíbrio hiperbólico do tipo um $(4,48 ;-0,49)$ é o ponto de mínimo da função energia $V$ na fronteira da região de estabilidade $\partial A(3,43 ;-0,49)$, e consequentemente $D(-1,55) \subset A(3,43 ;-0,49)$ onde $D(-1,55)=\left\{(x ; y) \in \mathbb{R}^{2}: V(x ; y)<\right.$ $-1,55\}$, confirmando os resultados do Teorema 9.4.2, ver Figura 9.3. Tomando $B=$ $-1,4>-1,5577$, a componente conexa $D(-1,4)$ do conjunto de nível $\left\{(x, y) \in \mathbb{R}^{2}\right.$ : $V(x ; y)<-1,4\}$ contendo o equilíbrio $(3,43 ;-0,49)$ intercepta o complementar da região de estabilidade $A^{c}(3,43 ;-0,49)$, de acordo com o item $(i i)$ do Teorema 9.4.2, ver Figura 9.4 .

Exemplo 11.2.2 Considere o sistema de equações diferenciais

$$
\begin{aligned}
& \dot{x}=-y \\
& \dot{y}=\sin (3 x)-\cos (2 x)-y
\end{aligned}
$$

$\operatorname{com}(x, y) \in \mathbb{R}^{2}$.

O sistema (9.3) possui um ponto de equilíbrio hiperbólico assintoticamente estável $x^{s}=(0,31 ; 0)$ e três pontos de equilíbrio instáveis, são eles: $p=(1,57 ; 0)$ um ponto de equilíbrio sela-nó do tipo zero, $x^{1}=(2,82 ; 0)$ e $x^{2}=(-0,99 ; 0)$ pontos de equilíbrio hiperbólicos do tipo um. Os três pontos de equilíbrio instáveis $p, x^{1}$ e $x^{2}$ pertencem à fronteira da região de estabilidade $\partial A(0,31 ; 0)$ que é composta pela união das va-riedades estáveis dos três equilíbrios, de acordo com o Teorema 9.3.3, ver Figura 9.5. A função $V(x ; y)=-\frac{\cos (3 x)}{3}-\frac{\sin (2 x)}{2}+\frac{y^{2}}{2}$ é uma função energia do sistema (9.3). A função ener- 


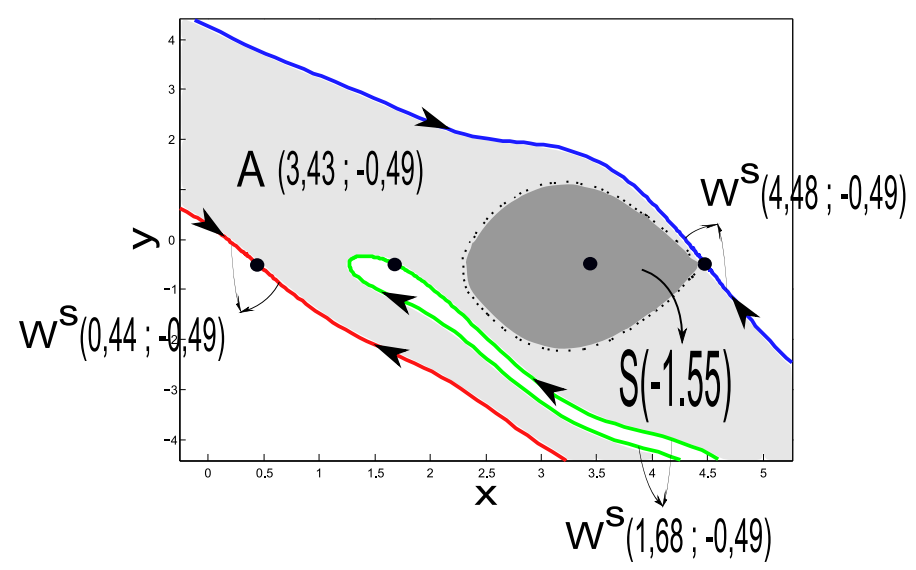

Figura 9.3: O retrato de fase do sistema (9.2). A região de estabilidade do ponto de equilíbrio assintoticamente estável $(3,43 ;-0,49)$ é representada pela área em cinza claro. A componente conexa $D(-1,55)$ do conjunto de nível $\left\{(x, y) \in \mathbb{R}^{2}: V(x ; y)<-1,55\right\}$ contendo o equilíbrio $(3,43 ;-0,49)$ representada pela área em cinza escuro é uma estimativa da região de estabilidade.

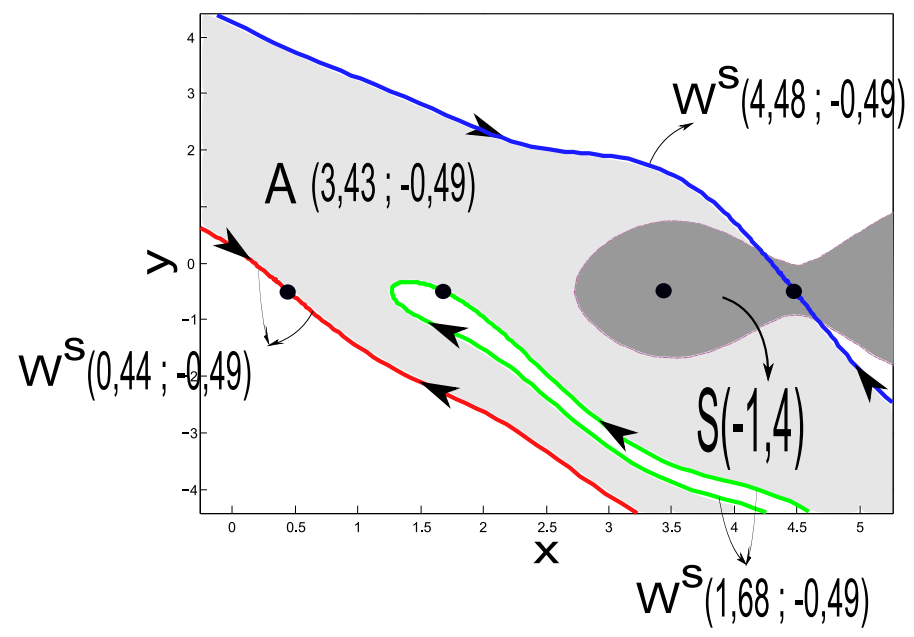

Figura 9.4: O retrato de fase do sistema (9.2). A região de estabilidade do ponto de equilíbrio assintoticamente estável $(3,43 ;-0,49)$ é representada pela área em cinza claro. A componente conexa $D(-1,4)$ do conjunto de nível $\left\{(x, y) \in \mathbb{R}^{2}: V(x ; y)<-1,4\right\}$ contendo o equilíbrio $(3,43 ;-0,49)$ representada pela área em cinza escuro intercepta $o$ complementar da região de estabilidade $A^{c}(3,43 ;-0,49)$.

gia $V(x ; y ;)$ é tal que $V(1,57 ; 0)=0<0,48=V(2,82 ; 0)<V(-0,99 ; 0)=0,79$, logo o ponto de equilíbrio não hiperbólico sela-nó do tipo zero $(1,57 ; 0)$ é o ponto de mínimo da função energia $V$ na fronteira da região de estabilidade $\partial A(0,31 ; 0)$, e consequentemente $D(0) \subset A(0,31 ; 0)$ onde $D(0)=\left\{x \in \mathbb{R}^{n}: V(x)<0\right\}$, confirmando os resultados do Teorema 9.4.2, ver Figura 9.5. Tomando $B=0,2>0$, a componente conexa $D(0,2)$ do conjunto de nível $\left\{(x, y) \in \mathbb{R}^{2}: V(x ; y)<0,2\right\}$ contendo o equilíbrio $(0,31 ; 0)$ intercepta o complementar da região de estabilidade $A^{c}(0,31 ; 0)$, de acordo com o item (ii) do Teorema 9.4.2, ver Figura 9.6 


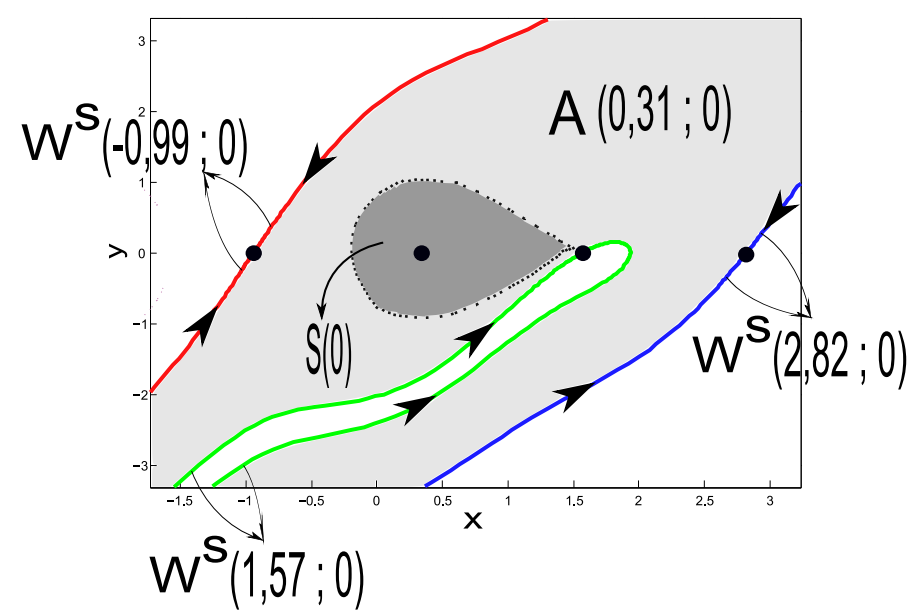

Figura 9.5: O retrato de fase do sistema (9.3). A região de estabilidade do ponto de equilíbrio assintoticamente estável $(0,31 ; 0)$ é representada pela área em cinza claro. A componente conexa $D(0)$ do conjunto de nível $\left\{(x, y) \in \mathbb{R}^{2}: V(x ; y)<0\right\}$ contendo o equilíbrio $(0,31 ; 0)$ representada pela área em cinza escuro é uma estimativa da região de estabilidade.

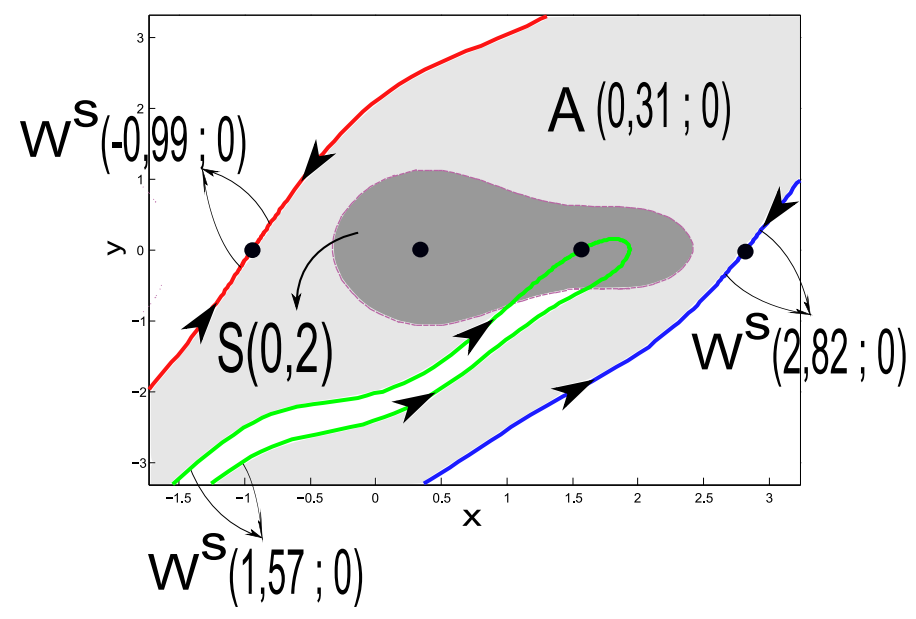

Figura 9.6: O retrato de fase do sistema (9.3). A região de estabilidade do ponto de equilíbrio assintoticamente estável $(0,31 ; 0)$ é representada pela área em cinza claro. A componente conexa $D(0,2)$ do conjunto de nível $\left\{(x, y) \in \mathbb{R}^{2}: V(x ; y)<0,2\right\}$ contendo o equilíbrio $(0,31 ; 0)$ representada pela área em cinza escuro intercepta o complementar da região de estabilidade $A^{c}(0,31 ; 0)$.

\subsection{Comportamento das estimativas da região de estabilidade próx- imo a um parâmetro de bifurcação sela-nó do tipo zero}

Nesta seção, apresentaremos resultados que permitem entender o comportamento das estimativas da região de estabilidade próximo a um parâmetro de bifurcação sela-nó do tipo zero. Ofereceremos um algoritmo conceitual para obter estimativas da região de estabilidade perturbada via conjunto de nível de uma dada função energia na vizinhança de um parâmetro de bifurcação sela-nó do tipo zero.

Considere a seguinte suposição sobre a família de equações diferenciais (7.1). 
$\left(\mathbf{A 3}^{\prime}\right)$ Existe uma função $V(x, \lambda)$ associada a família de equações diferenciais (7.1) onde $(x, \lambda) \mapsto V(x, \lambda)$ é de classe $C^{1}$ em $\mathbb{R}^{n} \times \mathbb{R}$ e $V_{\lambda}()=.V(., \lambda)$ é uma função energia do sistema $\dot{x}=f_{\lambda}(x)$ para cada $\lambda$ fixo.

A suposição $\left(A 3^{\prime}\right)$ é importante para estudarmos o comportamento do ponto de mínimo de uma função energia na fronteira da região de estabilidade sob a influência das variações dos parâmetros.

Observação 9.5.1. Pelo Teorema 9.3.2 podemos afirmar que a suposição $\left(A 3^{\prime}\right)$ é uma condição suficiente para a satisfação da suposição (A3) para todo $\lambda$, consequentemente os Teoremas 8.1.1, 8.1.2, 8.1.3 e Corolário 8.1.2 continuam verdadeiros substituindo a suposição $(A 3)$ por $\left(A 3^{\prime}\right)$.

O teorema a seguir descreve o comportamento do ponto de mínimo de uma função energia na fronteira da região de estabilidade para variações dos parâmetros próximo a um parâmetro de bifurcação sela-nó do tipo zero.

Teorema 9.5.1. ( Persistência do ponto de mínimo na fronteira da região de estabilidade): Seja $\left(x_{\lambda_{0}}, \lambda_{0}\right)$ um ponto de bifurcação sela-nó do tipo zero de (7.1). Suponha que o ponto de equilíbrio sela-nó do tipo zero $x_{\lambda_{0}}$ pertença à fronteira da região de estabilidade $\partial A_{\lambda_{0}}\left(x_{\lambda_{0}}^{s}\right)$ de um ponto de equilíbrio hiperbólico assintoticamente estável $x_{\lambda_{0}}^{s}$ de (7.1) para $\lambda=\lambda_{0}$. Admita que as suposições (A2) e (A3') sejam satisfeitas em um intervalo aberto contendo o valor de bifurcação sela-nó do tipo zero $\lambda_{0}$ e a suposição (A4) está satisfeita para $\lambda=\lambda_{0}$. Assuma que o número de pontos de equilíbrio hiperbólicos do sistema (7.1) é finito e $x_{\lambda_{0}}$ é o único ponto de equilíbrio não hiperbólico para $\lambda=\lambda_{0}$. Suponha também, que para todo $\lambda \in I$, todos os pontos de equilíbrio do sistema perturbado $\dot{x}=f(x, \lambda)$ são pontos de equilíbrio perturbados originados do sistema $\dot{x}=f\left(x, \lambda_{0}\right)$. Sejam $x_{\lambda_{0}^{i}}, i=1, \ldots, k$ os pontos de equilíbrio hiperbólicos na fronteira $\partial A_{\lambda_{0}}\left(x_{\lambda_{0}}^{s}\right) e V_{\lambda_{0}}\left(x_{\lambda_{0}}\right) \neq V_{\lambda_{0}}\left(x_{\lambda_{0}^{i}}\right) \neq V_{\lambda_{0}}\left(x_{\lambda_{0}^{j}}\right)$ para todo $i, j=1,2, \ldots, k$ com $i \neq j$. Então as seguintes afirmações são verdadeiras:

(i) Se $x_{\lambda_{0}^{1}}$ é o ponto de mínimo da função energia $V_{\lambda_{0}}$ na fronteira $\partial A_{\lambda_{0}}\left(x_{\lambda_{0}}^{s}\right)$, então existe $\zeta>0$ tal que o ponto de equilíbrio perturbado $x_{\lambda 1}$ é o ponto de mínimo da função energia $V_{\lambda}$ na fronteira $\partial A_{\lambda}\left(x_{\lambda}^{s}\right)$ para todo $\lambda \in\left(\lambda_{0}-\zeta, \lambda_{0}+\zeta\right)$.

(ii) Se o ponto de equilíbrio sela-nó do tipo zero $x_{\lambda_{0}}$ é o ponto de mínimo da função energia $V_{\lambda_{0}}$ na fronteira $\partial A_{\lambda_{0}}\left(x_{\lambda_{0}}^{s}\right)$, então existe $\eta>0$ tal que o ponto de equilíbrio $y_{\lambda}^{u}$, originado da bifurcação sela-nó do tipo zero, é o ponto de mínimo da função energia $V_{\lambda}$ na fronteira $\partial A_{\lambda}\left(x_{\lambda}^{s}\right)$ para todo $\lambda \in\left(\lambda_{0}-\eta, \lambda_{0}\right)$.

Demonstração. (i) Sejam $x_{\lambda_{0}^{i}}, i=1, \ldots, k$ os pontos de equilíbrio hiperbólicos na fronteira $\partial A_{\lambda_{0}}\left(x_{\lambda_{0}}^{s}\right)$ e $x_{\lambda_{0}^{r}}, r=k+1, \ldots, m$ os pontos de equilíbrio hiperbólicos na fronteira da região de estabilidade fraca $\partial S_{\lambda_{0}}^{*}\left(x_{\lambda_{0}}\right)$. O Corolário 8.1.2 garante que existe $\varepsilon>0$ tal que os 
pontos de equilíbrio perturbados $x_{\lambda d}, d=1, \ldots, k, k+1, \ldots, m$ são os pontos de equilíbrio que pertencem à fronteira $\partial A_{\lambda}\left(x_{\lambda}^{s}\right)$ para todo $\lambda \in\left(\lambda_{0}, \lambda_{0}+\varepsilon\right)$. Se $x_{\lambda_{0}^{1}}$ é o ponto de mínimo da função energia $V_{\lambda_{0}}$ na fronteira $\partial A_{\lambda_{0}}\left(x_{\lambda_{0}}^{s}\right)$ e como $V_{\lambda_{0}}\left(x_{\lambda_{0}}\right) \neq V_{\lambda_{0}}\left(x_{\lambda_{0}^{i}}\right) \neq V_{\lambda_{0}}\left(x_{\lambda_{0}^{j}}\right)$ para todo $i, j=1,2, \ldots, k$ com $i \neq j$, então $V_{\lambda_{0}}\left(x_{\lambda_{0}^{1}}\right)<V_{\lambda_{0}}\left(x_{\lambda_{0}^{b}}\right)$ para todo $b=2, \ldots, k \mathrm{e}$ $V_{\lambda_{0}}\left(x_{\lambda_{0}^{1}}\right)<V_{\lambda_{0}}\left(x_{\lambda_{0}}\right)$. Por outro lado, $V_{\lambda_{0}}\left(x_{\lambda_{0}}\right) \leqslant V_{\lambda_{0}}\left(x_{\lambda_{0}^{r}}\right)$ para todo $r=k+1, \ldots, m$, pois $x_{\lambda_{0}^{r}} \in \partial S_{\lambda_{0}}^{*}\left(x_{\lambda_{0}}\right)$. Assim $V_{\lambda_{0}}\left(x_{\lambda_{0}^{1}}\right)<V_{\lambda_{0}}\left(x_{\lambda_{0}^{q}}\right)$ para todo $q=2, \ldots, k, k+1, \ldots, m$. Explorando o fato que, $V(x, \lambda)$ é uma função continua em $\mathbb{R}^{n} \times I$, podemos afirmar que existe $\delta>$ 0 tal que $V_{\lambda}\left(x_{\lambda^{1}}\right)<V_{\lambda}\left(x_{\lambda q}\right)$ para todo $\lambda \in\left(\lambda_{0}-\delta, \lambda_{0}+\delta\right)$ e $q=2, \ldots, k, k+1, \ldots, m$. Tomando $\zeta=\min \left\{\varepsilon, \delta\right.$, temos em particular que $x_{\lambda^{d}}, d=1, \ldots, k, k+1, \ldots, m$ são os pontos de equilíbrio na fronteira $\partial A_{\lambda}\left(x_{\lambda}^{s}\right)$ e $V_{\lambda}\left(x_{\lambda^{1}}\right)<V_{\lambda}\left(x_{\lambda q}\right)$ para todo $\lambda \in\left(\lambda_{0}, \lambda_{0}+\zeta\right)$ e $q=2, \ldots, k, k+1, \ldots, m$. Portanto, o Teorema 9.4.1 garante que o ponto de equilíbrio perturbado $x_{\lambda^{1}}$ é o ponto de mínimo da função energia $V_{\lambda}$ na fronteira $\partial A_{\lambda}\left(x_{\lambda}^{s}\right)$ para todo $\lambda \in\left(\lambda_{0}, \lambda_{0}+\zeta\right)$. A prova que $x_{\lambda^{1}}$ é o ponto de mínimo da função energia $V_{\lambda}$ na fronteira $\partial A_{\lambda}\left(x_{\lambda}^{s}\right)$ para todo $\lambda \in\left(\lambda_{0}-\zeta, \lambda_{0}\right)$ é similar.

(ii) O Corolário 8.1.2 garante que existe $\varepsilon>0$ tal que o ponto de equilíbrio $y_{\lambda}^{u}$ originado da bifurcação sela-nó do tipo zero e os pontos de equilíbrio perturbados $x_{\lambda^{i}}, i=1, \ldots, k$ são os pontos de equilíbrio que pertencem à fronteira $\partial A_{\lambda}\left(x_{\lambda}^{s}\right)$ para todo $\lambda \in\left(\lambda_{0}-\varepsilon, \lambda_{0}\right)$. Se $x_{\lambda_{0}}$ é o ponto de mínimo da função energia $V_{\lambda_{0}}$ na fronteira $\partial A_{\lambda_{0}}\left(x_{\lambda_{0}}^{s}\right)$ e como $V_{\lambda_{0}}\left(x_{\lambda_{0}}\right) \neq$ $V_{\lambda_{0}}\left(x_{\lambda_{0}^{i}}\right)$ para todo $i=1, \ldots, k$, então $V_{\lambda_{0}}\left(x_{\lambda_{0}}\right)<V_{\lambda_{0}}\left(x_{\lambda_{0}^{i}}\right)$ para todo $i=1, \ldots, k$. Explorando o fato que, $V(x, \lambda)$ é uma função continua em $\mathbb{R}^{n} \times I$, podemos afirmar que existe $\delta>0$ tal que $V_{\lambda}\left(y_{\lambda}^{u}\right)<V_{\lambda}\left(x_{\lambda^{i}}\right)$ para todo $\lambda \in\left(\lambda_{0}-\delta, \lambda_{0}\right)$ e $i=1, \ldots, k$. Tomando $\zeta=\min \{\varepsilon, \delta\}$, temos em particular que $y_{\lambda}^{u}$ e $x_{\lambda^{i}}, i=1, \ldots, k$ são os pontos de equilíbrio que pertencem à fronteira $\partial A_{\lambda}\left(x_{\lambda}^{s}\right)$ e $V_{\lambda}\left(y_{\lambda}^{u}\right)<V_{\lambda}\left(x_{\lambda^{i}}\right)$ para todo $\lambda \in\left(\lambda_{0}-\zeta, \lambda_{0}\right)$ e $i=1, \ldots, k$. Portanto, o Teorema 9.4.1 garante que o ponto de equilíbrio $y_{\lambda}^{u}$ é o ponto de mínimo da função energia $V_{\lambda}$ na fronteira $\partial A_{\lambda}\left(x_{\lambda}^{s}\right)$ para todo $\lambda \in\left(\lambda_{0}-\zeta, \lambda_{0}\right)$.

No caso particular que todos os pontos de equilíbrio na fronteira da região de estabilidade são hiperbólicos, a persistência do ponto de mínimo da função energia na fronteira da região de estabilidade é um caso especial do Teorema 9.5.1.

O Teorema 9.5.1 é importante do ponto de vista computacional, pois, uma vez conhecido o ponto de mínimo na fronteira da região de estabilidade, ele indica onde procurar o ponto de mínimo na fronteira da região de estabilidade perturbada, incluindo o caso quando uma bifurcação sela-nó do tipo zero ocorre na fronteira da região de estabilidade.

Como uma consequência dos Teoremas 8.1.2, 9.4.2 e 9.5.1, obtemos o seguinte corolário que permite entender o comportamento da estimativa da região de estabilidade proposta pelo Teorema 9.4.2 para variações dos parâmetros próximo a um parâmetro de bifurcação sela-nó do tipo zero.

Corolário 9.5.1. ( Estimativas e uniforme da região de estabilidade na vizi-nhança de um parâmetro de bifurcação sela-nó do tipo zero): Seja $\left(x_{\lambda_{0}}, \lambda_{0}\right)$ um ponto de bifurcação 
sela-nó do tipo zero de (7.1). Suponha que o ponto de equilíbrio sela-nó do tipo zero $x_{\lambda_{0}}$ pertença à fronteira da região de estabilidade $\partial A_{\lambda_{0}}\left(x_{\lambda_{0}}^{s}\right)$ de um ponto de equilíbrio hiperbólico assintoticamente estável $x_{\lambda_{0}}^{s}$ de (7.1) para $\lambda=\lambda_{0}$. Admita que as suposições $(A 2) e$ $\left(A 3^{\prime}\right)$ sejam satisfeitas em um intervalo aberto contendo o valor de bifurcação sela-nó do tipo zero $\lambda_{0}$ e a suposição (A4) está satisfeita para $\lambda=\lambda_{0}$. Assuma que o número de pontos de equilíbrio hiperbólicos do sistema (7.1) é finito e $x_{\lambda_{0}}$ é o único ponto de equilíbrio não hiperbólico para $\lambda=\lambda_{0}$. Suponha também, que para todo $\lambda \in I$, todos os pontos de equilíbrio do sistema perturbado $\dot{x}=f(x, \lambda)$ são pontos de equilíbrio perturbados originados do sistema $\dot{x}=f\left(x, \lambda_{0}\right)$. Sejam $x_{\lambda_{0}^{i}}, i=1, \ldots, k$ os pontos de equilíbrio hiperbólicos na fronteira $\partial A_{\lambda_{0}}\left(x_{\lambda_{0}}^{s}\right)$ e $V_{\lambda_{0}}\left(x_{\lambda_{0}}\right) \neq V_{\lambda_{0}}\left(x_{\lambda_{0}^{i}}\right) \neq V_{\lambda_{0}}\left(x_{\lambda_{0}^{j}}\right)$ para todo $i, j=1,2, \ldots, k$ com $i \neq j$. Então as seguintes afirmações são verdadeiras:

(i) Se $x_{\lambda_{0}}$ é o ponto de mínimo da função energia $V_{\lambda_{0}}$ na fronteira $\partial A_{\lambda_{0}}\left(x_{\lambda_{0}}^{s}\right)$, então existe $\zeta>0$ tal que $D_{\lambda}\left(L_{\lambda}\right) \subset A_{\lambda}\left(x_{\lambda}^{s}\right)$ onde $L_{\lambda}=V_{\lambda}\left(x_{\lambda 1}\right)$, e $D_{\lambda}\left(B_{\lambda}\right) \cap A_{\lambda}^{c}\left(x_{\lambda}^{s}\right) \neq \emptyset$ para todo $B_{\lambda}>L_{\lambda}$ e $\lambda \in\left(\lambda_{0}-\zeta, \lambda_{0}+\zeta\right)$.

(ii) Se o ponto de equilíbrio sela-nó do tipo zero $x_{\lambda_{0}}$ é o ponto de mínimo da função energia $V_{\lambda_{0}}$ na fronteira $\partial A_{\lambda_{0}}\left(x_{\lambda_{0}}^{s}\right)$, então existe $\zeta>0$ tal que $D_{\lambda}\left(L_{\lambda}\right) \subset A_{\lambda}\left(x_{\lambda}^{s}\right)$ onde $L_{\lambda}=V_{\lambda}\left(y_{\lambda}^{u}\right)$, e $D_{\lambda}\left(B_{\lambda}\right) \cap A_{\lambda}^{c}\left(x_{\lambda}^{s}\right) \neq \emptyset$ para todo $B_{\lambda}>L_{\lambda}$ e $\lambda \in\left(\lambda_{0}-\zeta, \lambda_{0}\right)$.

(iii) Se o ponto de equilíbrio sela-nó do tipo zero $x_{\lambda_{0}}$ é o ponto de mínimo da função energia $V_{\lambda_{0}}$ na fronteira $\partial A_{\lambda_{0}}\left(x_{\lambda_{0}}^{s}\right)$, então existe $\zeta>0$ tal que para $L_{\lambda_{0}}=V_{\lambda_{0}}\left(x_{\lambda_{0}}\right)$, o conjunto $D_{\lambda_{0}}\left(L_{\lambda_{0}}\right) \subset A_{\lambda}\left(x_{\lambda}^{s}\right)$ para todo $\lambda \in\left[\lambda_{0}, \lambda_{0}+\zeta\right)$.

Observação 9.5.2. Para cada $\lambda$ fixo, o conjunto $D_{\lambda}\left(L_{\lambda}\right)=\left\{x \in \mathbb{R}^{n}: V_{\lambda}(x)<L_{\lambda}\right\} e$ $D_{\lambda}\left(B_{\lambda}\right)=\left\{x \in \mathbb{R}^{n}: V_{\lambda}(x)<B_{\lambda}\right\}$, onde $L_{\lambda}$ e $B_{\lambda}$ são números reais.

Baseado nos Teoremas 9.4.2, 9.5.1 e Corolário 9.5.1, propomos abaixo um algoritmo conceitual para obter estimativas da região de estabilidade perturbada $A_{\lambda}\left(x_{\lambda}^{s}\right)$ via conjunto de nível de uma dada função energia na vizinhança de um parâmetro de bifurcação sela-nó do tipo zero.

\section{Algoritmo: estimativa da Região de Estabilidade Perturbada com Bifurcações Sela-Nó do Tipo Zero na Fronteira}

1. Para um certo valor fixo do parâmetro $\lambda=\lambda_{0}$, calcule todos os pontos de equilíbrio $x_{\lambda_{0}^{i}}$, $i=1, \ldots, k$ na fronteira da região de estabilidade do ponto de equilíbrio assintoticamente estável $x_{\lambda_{0}}^{s}$.

2. Calcule as energias $V_{\lambda_{0}}\left(x_{\lambda_{0}^{i}}\right)$ para todo $i=1, \ldots, k$.

3. Identifique o ponto de equilíbrio com menor energia na fronteira da região de estabilidade do ponto de equilíbrio assintoticamente estável $x_{\lambda_{0}}^{s}$. Seja ele $x_{\min _{\lambda_{0}}}$.

4. A componente conexa do conjunto de nível $\left\{x \in \mathbb{R}^{n}: V_{\lambda_{0}}(x)<V_{\lambda_{0}}\left(x_{\min _{\lambda_{0}}}\right)\right\}$ contendo o ponto de equilíbrio assintoticamente estável $x_{\lambda_{0}}^{s}$ é uma estimativa da região de estabilidade $A_{\lambda_{0}}\left(x_{\lambda_{0}}^{s}\right)$ na forma de um conjunto de nível da função $V_{\lambda_{0}}$.

5. Se $x_{\min _{\lambda_{0}}}$ é um ponto de equilíbrio hiperbólico, então a componente conexa do conjunto 
de nível $\left\{x \in \mathbb{R}^{n}: V_{\lambda}(x)<V_{\lambda}\left(x_{\min _{\lambda}}\right)\right\}$ contendo o ponto de equilíbrio assintoticamente estável $x_{\lambda}^{s}$ é uma estimativa da região de estabilidade $A_{\lambda}\left(x_{\lambda}^{s}\right)$ do sistema perturbado numa vizinhança de $\lambda_{0}$.

6. Se $x_{\min _{\lambda_{0}}}$ é um ponto de equilíbrio sela-nó do tipo zero, então:

(i) a componente conexa do conjunto de nível $\left\{x \in \mathbb{R}^{n}: V_{\lambda}(x)<V_{\lambda}\left(y_{\lambda}^{u}\right)\right\}$ contendo o ponto de equilíbrio assintoticamente estável $x_{\lambda}^{s}$ é uma estimativa da região de estabilidade $A_{\lambda}\left(x_{\lambda}^{s}\right)$ do sistema perturbado para $\lambda<\lambda_{0}$ numa vizinhança de $\lambda_{0}$, onde $y_{\lambda}^{u}$ é o ponto de equilíbrio instável originado da bifurcação sela-nó do tipo zero.

(ii) a componente conexa do conjunto de nível $\left\{x \in \mathbb{R}^{n}: V_{\lambda_{0}}(x)<V_{\lambda_{0}}\left(x_{\min _{\lambda_{0}}}\right)\right\}$ é uma estimativa uniforme da região de estabilidade de $x_{\lambda}^{s}$ para $\lambda>\lambda_{0}$ numa vizinhança de $\lambda_{0}$.

Observação 9.5.3. Quando o ponto de mínimo da função energia na fronteira da região de estabilidade é hiperbólico, o nível ótimo de energia para estimar a região de estabilidade perturbada é o nível de energia calculado no ponto de equilíbrio hiperbólico perturbado. Quando o ponto de mínimo da função energia na fronteira da região de estabilidade é o ponto de equilíbrio sela-nó do tipo zero $x_{\lambda_{0}}$, o nível ótimo de energia para estimar a região de estabilidade perturbada para $\lambda<\lambda_{0}$ é calculado no ponto de equilíbrio instável $y_{\lambda}^{u}$, originado da bifurcação sela-nó do tipo zero. Para $\lambda>\lambda_{0}$ o nível de energia $L_{\lambda_{0}}=V_{\lambda_{0}}\left(x_{\lambda_{0}}\right)$ fornece uma estimativa da região de estabilidade perturbada, porém o nível de energia $L_{\lambda_{0}}=V_{\lambda_{0}}\left(x_{\lambda_{0}}\right)$ não é ótimo para estimar a região de estabilidade perturbada $A_{\lambda}\left(x_{\lambda}^{s}\right)$. Usar o nível de energia $L_{\lambda_{0}}=V_{\lambda_{0}}\left(x_{\lambda_{0}}\right)$ para estimar a região de estabilidade perturbada é justificável quando deseja-se evitar o esforço computacional do cálculo do novo ponto de mínimo na fronteira da região de estabilidade perturbada para $\lambda>\lambda_{0}$.

Os exemplos a seguir ilustram os resultados obtidos nesta seção.

Exemplo 11.3.1 Considere o sistema de equações diferenciais

$$
\begin{aligned}
& \dot{x}=-y \\
& \dot{y}=-x^{4}+x^{2}-y+\lambda
\end{aligned}
$$

$\operatorname{com}(x, y) \in \mathbb{R}^{2}$ e $\lambda \in \mathbb{R}$.

A função $V(x ; y ; \lambda)=-\frac{x^{5}}{5}+\frac{x^{3}}{3}+\frac{y^{2}}{2}+\lambda x$ é uma função energia para o sistema (9.4) e de classe $C^{1}$ em $\mathbb{R}^{2} \times \mathbb{R}$. O sistema (9.4) possui, para $\lambda_{0}=0$, três pontos de equilíbrio; são eles $x_{\lambda_{0}}^{s}=(-1 ; 0)$, um ponto de equilíbrio hiperbólico assintoticamente estável, $x_{\lambda_{0}}=(0 ; 0)$, um ponto de equilíbrio sela-nó do tipo zero e $x_{\lambda_{0}}^{*}=(1 ; 0)$, um ponto de equilíbrio hiperbólico instável. O ponto de equilíbrio sela-nó do tipo zero $x_{\lambda_{0}}$ pertence à fronteira $\partial A_{\lambda_{0}}(-1 ; 0)$ e o ponto de equilíbrio hiperbólico instável $x_{\lambda_{0}}^{*}$ pertence à fronteira da região de estabilidade fraca $\partial S^{*}(0 ; 0)$. A fronteira da região de estabi-lidade $\partial A_{\lambda_{0}}(-1 ; 0)$ é composta pela variedade estável do ponto de equilíbrio sela-nó do tipo zero $x_{\lambda_{0}}=(0 ; 0)$, de acordo com o Teorema 9.3.3, ver Figura 9.8. O ponto de equilíbrio $x_{\lambda_{0}}$ é o ponto de mínimo da função energia $V_{\lambda_{0}}$ na fronteira $\partial A_{\lambda_{0}}(-1 ; 0)$ e consequentemente 
$D(0) \subset A_{\lambda_{0}}(-1 ; 0)$ onde $V_{\lambda_{0}}\left(x_{\lambda_{0}}\right)=0$, confirmando o Teorema 9.4.2, ver Figura 9.8. Para $\lambda=-0,1$, o sistema (9.4) possui quatro pontos de equilíbrio; são eles $x_{\lambda}^{s}=(-0,94 ; 0)$, um ponto de equilíbrio hiperbólico assintoticamente estável, $x_{\lambda}^{*}=(0,94 ; 0)$ um ponto de equilíbrio hiperbólico instável, $y_{\lambda}^{u}=(-0,33 ; 0)$ um ponto de equilíbrio hiperbólico instável e $y_{\lambda}^{s}=(0,33 ; 0)$ um ponto de equilíbrio hiperbólico assintoticamente estável. Os pontos de equilíbrio $y_{\lambda}^{u}$ e $y_{\lambda}^{s}$ são originados do ponto de equilíbrio sela-nó do tipo zero $x_{\lambda_{0}}$ em uma bifurcação sela-nó do tipo zero. O ponto de equilíbrio $y_{\lambda}^{u}$ pertence à fronteira $\partial A_{\lambda}(-0,94 ; 0) \cap \partial A_{\lambda}(0,33 ; 0)$. O ponto de mínimo da função energia $V_{\lambda}$ na fronteira $\partial A_{\lambda}(-0,94 ; 0)$ é $y_{\lambda}^{u}$, confirmando os resultados do Teorema 9.5.1. Além disso, $D(-0,09) \subset A_{\lambda}(-0,94 ; 0)$ onde $V_{\lambda}\left(y_{\lambda}^{u}\right)=-0,09$, de acordo com o algoritmo descrito anteriormente, ver Figura 9.7. Para $\lambda=0,1$, o sistema (9.4) possui dois pontos de equilíbrio; são eles $x_{\lambda}^{s}=(-1,04 ; 0)$ um ponto de equilíbrio hiperbólico assintoticamente estável e $x_{\lambda}^{*}=(1,04 ; 0)$ um ponto de equilíbrio hiperbólico instável que pertence à fronteira da região de estabilidade $\partial A_{\lambda}(-1,04 ; 0)$. Usando o nível de energia $V_{\lambda_{0}}\left(x_{\lambda_{0}}\right)=0$ ainda temos que $D(0) \subset A_{\lambda}(-1,04 ; 0)$, de acordo com o algoritmo descrito anteriormente, ver Figura 9.9.

Exemplo 11.3.2 Considere o sistema de equações diferenciais

$$
\begin{aligned}
& \dot{x}=y+\lambda \\
& \dot{y}=\sin (3 x-1)-\cos (2 x-1,5)-y
\end{aligned}
$$

$\operatorname{com}(x, y) \in \mathbb{R}^{2}$ e $\lambda \in \mathbb{R}$.

A função $V(x ; y ; \lambda)=\frac{\cos (3 x-1)}{3}+\frac{\sin (2 x-1.5)}{2}+\lambda(y-x)+\frac{y^{2}}{2}$ é uma função energia para o sistema (9.5) e de classe $C^{1}$ em $\mathbb{R}^{2} \times \mathbb{R}$. Para $\lambda_{0}=0,49$, vimos que o sistema (9.5) possui um ponto de equilíbrio hiperbólico assintoticamente estável $x_{\lambda_{0}}^{s}=(3,43 ;-0,49)$ e três equilíbrios instáveis, são eles; $x_{\lambda_{0}}=(1,68 ;-0,49)$ um ponto de equilíbrio selanó do tipo zero, $x_{\lambda_{0}^{1}}=(4,48 ;-0,49)$ e $x_{\lambda_{0}^{2}}=(0,44 ;-0,49)$ pontos de equilíbrio hiperbólicos do tipo um. Os três pontos de equilíbrio instáveis $x_{\lambda_{0}}, x_{\lambda_{0}^{1}}$ e $x_{\lambda_{0}^{2}}$ pertencem à fronteira $\partial A_{\lambda_{0}}(3,43 ;-0,49)$ que é composta pela união das variedades estáveis dos três equilíbrios, de acordo com o Teorema 9.3.3, ver Figura 9.11. A função energia $V\left(x ; y ; \lambda_{0}\right)$ é tal que $V(4,48 ;-0,49 ; 0,49)=-1,55<-0,68=V(1,68 ;-0,49 ; 0,49)<$ $V(0,44 ;-0,49 ; 0,49)=-0,31$. Portanto, pelo Teorema 9.4 .1 o ponto de equilíbrio do tipo um $(4,48 ;-0,49)$ é o ponto de mínimo da função energia $V_{\lambda_{0}}$ na fronteira $\partial A_{\lambda_{0}}(3,43 ;-0,49)$, e consequentemente $D(-1,55) \subset A_{\lambda_{0}}(3,43 ;-0,49)$ confirmando o Teorema 9.4.2, ver Figura 9.11. Para $\lambda=0,48$, o ponto de equilíbrio hiperbólico assintoticamente estável perturbado é $x_{\lambda}^{s}=(3,43 ;-0,48)$. Na fronteira da região de estabilidade $\partial A_{\lambda}(3,43 ;-0,48)$ existem três pontos de equilíbrio; são eles $x_{\lambda 1}=(4,48 ;-0,48)$, e $x_{\lambda^{2}}=(0,45 ;-0,48)$ os pontos de equilíbrio hiperbólicos instáveis perturbados e $y_{\lambda}^{u}=$ $(1,74 ;-0,48)$ um ponto de equilíbrio hiperbólico instável. O ponto de equilíbrio $y_{\lambda}^{u}$ é 
originado do ponto de equilíbrio sela-nó do tipo zero $x_{\lambda_{0}}$ em uma bifurcação sela-nó do tipo zero. O ponto de mínimo da função energia $V_{\lambda}$ na fronteira $\partial A_{\lambda}(3,43 ;-0,48)$ é $(4,48 ;-0,48)$, confirmando os resultados do Teorema 9.5.1. Além disso, $D(-1,47) \subset$ $A_{\lambda}(3,43 ;-0,48)$ onde $V(4,48 ;-0,48 ; 0,48)=-1,47$, de acordo com o algoritmo descrito anteriormente, ver Figura 9.10. Para $\lambda=0,51$, o ponto de equilíbrio hiperbólico assintoticamente estável perturbado é $x_{\lambda}^{s}=(3,43 ;-0,51)$. Na fronteira da região de estabi-lidade $\partial A_{\lambda}(3,43 ;-0,51)$ existem dois equilíbrios; são eles $x_{\lambda^{1}}=(4,47 ;-0,51)$ e $x_{\lambda^{2}}=(0,43 ;-0,51)$ os pontos de equilíbrio hiperbólicos instáveis perturbados. O ponto de mínimo da função energia $V_{\lambda}$ na fronteira da região de estabilidade perturbada $\partial A_{\lambda}(3,43 ;-0,51)$ é o ponto de equilíbrio instável $(4,47 ;-0,51)$ confirmando os resultados do Teorema 9.5.1. Além, $D(-1,64) \subset A_{\lambda}(3,43 ;-0,51)$ onde $V(4,47 ;-0,51,0,51)=$ $-1,64$, de acordo com o algoritmo descrito anteriormente, ver Figura 9.12.

Exemplo 11.3.3 Considere o sistema de equações diferenciais

$$
\begin{aligned}
& \dot{x}=-y \\
& \dot{y}=\sin (3 x)-\cos (2 x)-y+\lambda
\end{aligned}
$$

$\operatorname{com}(x, y) \in \mathbb{R}^{2}$ e $\lambda \in \mathbb{R}$.

A função $V(x ; y ; \lambda)=-\frac{\cos (3 x)}{3}-\frac{\sin (2 x)}{2}+\lambda x+\frac{y^{2}}{2}$ é uma função energia para o sistema (9.6) e de classe $C^{1}$ em $\mathbb{R}^{2} \times \mathbb{R}$. Para $\lambda_{0}=0$, vimos que o sistema (9.6) possui um ponto de equilíbrio hiperbólico assintoticamente estável $x_{\lambda_{0}}^{s}=(0,31 ; 0)$ e três pontos de equilíbrio instáveis, são eles; $x_{\lambda_{0}}=(1,57 ; 0)$ um ponto de equilíbrio sela-nó do tipo zero, $x_{\lambda_{0}^{1}}=(2,82 ; 0)$ e $x_{\lambda_{0}^{2}}=(-0,99 ; 0)$ pontos de equilíbrio hiperbólicos do tipo um. Os três pontos de equilíbrio instáveis $x_{\lambda_{0}}, x_{\lambda_{0}^{1}}$ e $x_{\lambda_{0}^{2}}$ pertencem à fronteira da região de estabilidade $\partial A_{\lambda_{0}}(0,31 ; 0)$ que é composta pela união das variedades estáveis dos três equilíbrios, de acordo com o Teorema 9.3.3, ver Figura 9.14. A função energia $V\left(x ; y ; \lambda_{0}\right)$ é tal que $V\left(x ; y ; \lambda_{0}\right)$ é tal que $V(1,5705 ; 0 ; 0)=0<0,48=V(2,82 ; 0 ; 0)<V(-0,99 ; 0 ; 0)=0,79$. Portanto, pelo Teorema 9.4.1 o ponto de equilíbrio não hiperbólico sela-nó do tipo zero $(1,57 ; 0)$ é o ponto de mínimo da função energia $V_{\lambda_{0}}$ na fronteira da região de estabilidade $\partial A_{\lambda_{0}}(0,31 ; 0)$, e consequentemente $D(0) \subset A_{\lambda_{0}}(0,31 ; 0)$ confirmando o Teorema 9.4.2, ver Figura 9.14. Para $\lambda=-0,02$, o ponto de equilíbrio hiperbólico assintoticamente estável perturbado é $x_{\lambda}^{s}=(0,32 ; 0)$. Na fronteira da região de estabilidade perturbada $\partial A_{\lambda}(0,32 ; 0)$ existem três pontos de equilíbrio; são eles $x_{\lambda^{1}}=(2,82 ; 0), x_{\lambda^{2}}=(-0,94 ; 0)$ os pontos de equilíbrio hiperbólicos instáveis perturbados e $y_{\lambda}^{u}=(1,48 ; 0)$ um ponto de equilíbrio hiperbólico instável. O ponto de equilíbrio $y_{\lambda}^{u}$ é originado do ponto de equilíbrio sela-nó do tipo zero $x_{\lambda_{0}}$ em uma bifurcação sela-nó do tipo zero. O ponto de mínimo da função energia $V_{\lambda}$ na fronteira perturbada $\partial A_{\lambda}(0,32 ; 0)$ é $y_{\lambda}^{u}$, confirmando os resultados do Teorema 9.5.1. Além disso, $D(-0,03) \subset A_{\lambda}(0,32 ; 0)$ onde $V(1,48 ; 0 ; 0)=-0,03$, de acordo com o algoritmo descrito anteriormente, ver Figura 9.13. Para $\lambda=0,02$, 
o ponto de equilíbrio hiperbólico assintoticamente estável perturbado é $x_{\lambda}^{s}=(0,3 ; 0)$. $\mathrm{Na}$ fronteira da região de estabilidade perturbada $\partial A_{\lambda}(0,3 ; 0)$ existem dois pontos de equilíbrio; são eles $x_{\lambda^{1}}=(2,83 ; 0)$ e $x_{\lambda^{2}}=(-0,93 ; 0)$, os pontos de equilíbrio hiperbólicos instáveis perturbados. Usando o nível de energia $V_{\lambda_{0}}\left(x_{\lambda_{0}}\right)=0$, ainda temos que $D(0) \subset A_{\lambda}(0,3 ; 0)$, de acordo com o algoritmo descrito anteriormente, ver Figura 9.15.

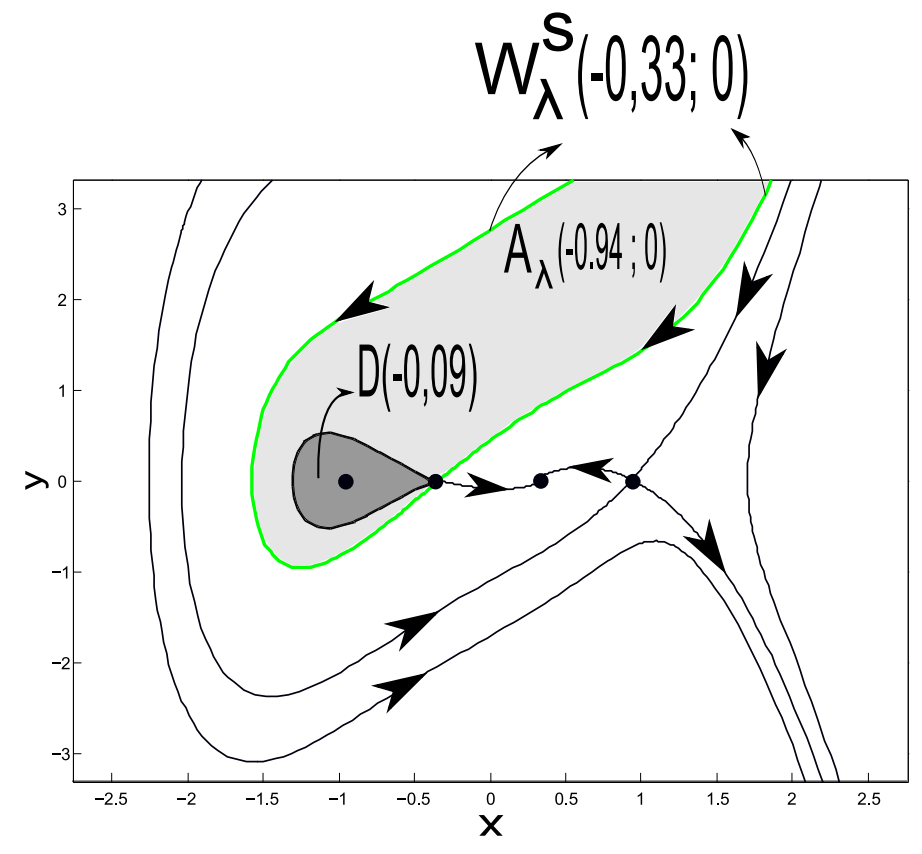

Figura 9.7: Retrato de fase do sistema (9.4) para $\lambda=-0,1$. A região de estabilidade do ponto de equilíbrio assintoticamente estável $(-0,94 ; 0)$ é representada pela área em cinza claro. A componente conexa $D(-0,09)$ do conjunto de nível $\left\{(x, y) \in \mathbb{R}^{2}: V_{\lambda}(x ; y)<\right.$ $-0,09\}$ contendo o equilíbrio $(-0,94 ; 0)$ representada pela área em cinza escuro é uma estimativa da região de estabilidade $A_{\lambda}(-0,94 ; 0)$. 


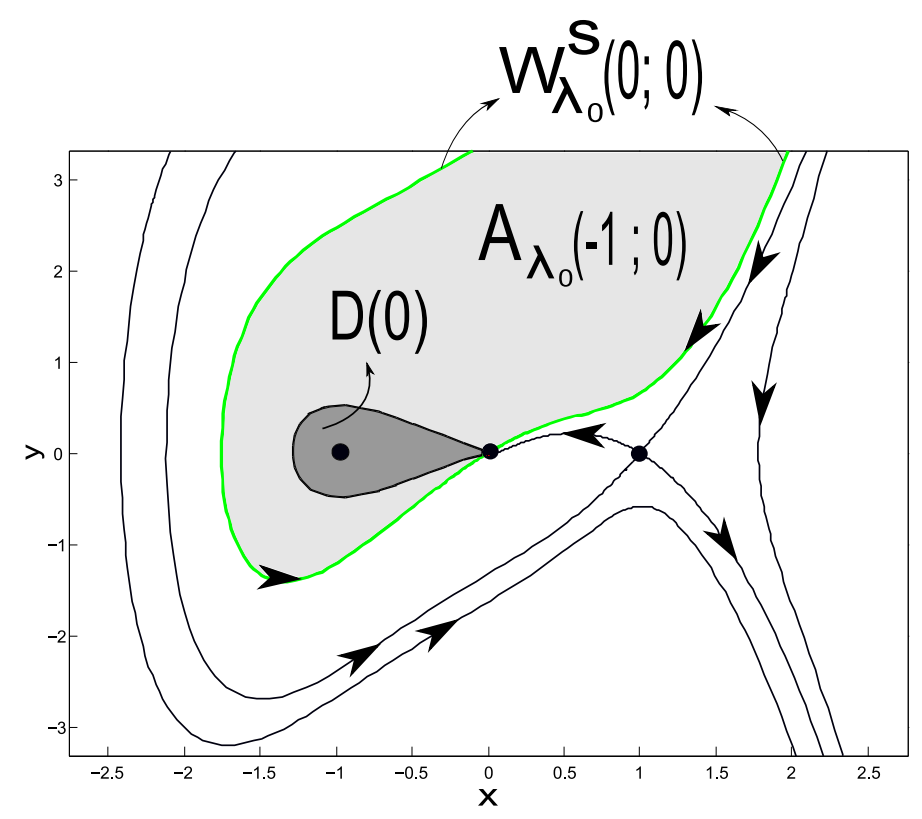

Figura 9.8: Retrato de fase do sistema (9.4) para $\lambda_{0}=0$. A região de estabilidade do ponto de equilíbrio assintoticamente estável $(-1 ; 0)$ é representada pela área em cinza claro. A componente conexa $D(0)$ do conjunto de nível $\left\{(x, y) \in \mathbb{R}^{2}: V_{\lambda_{0}}(x ; y)<0\right\}$ contendo o equilíbrio $(-1 ; 0)$ representada pela área em cinza escuro é uma estimativa da região de estabilidade $A_{\lambda_{0}}(-1 ; 0)$.

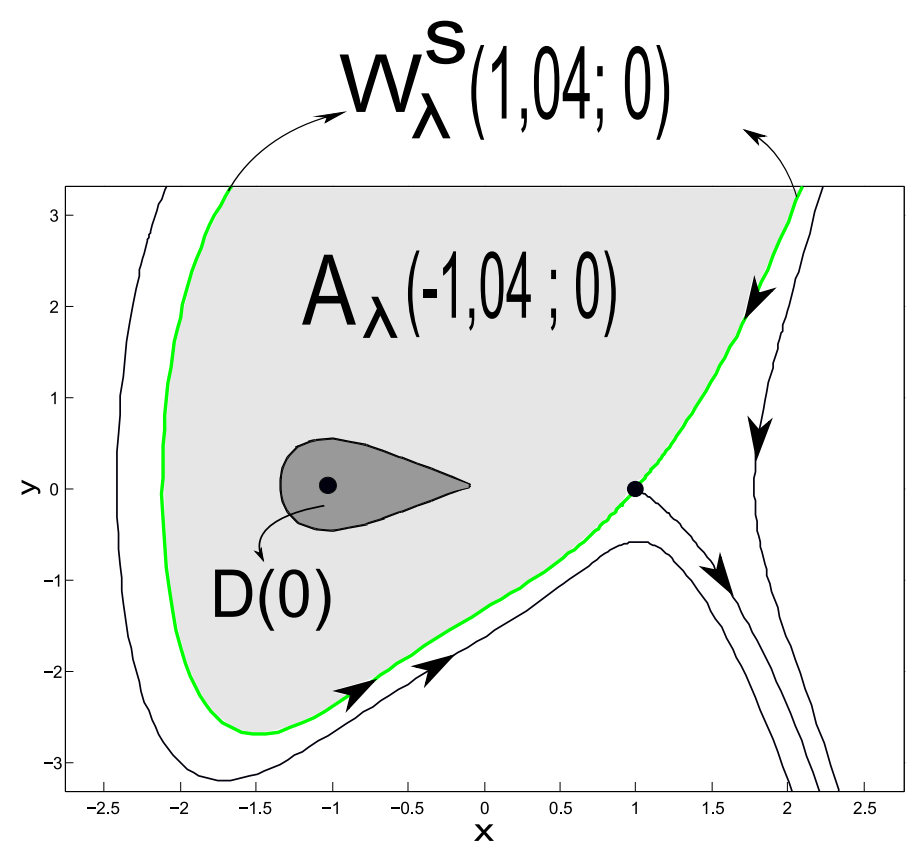

Figura 9.9: Retrato de fase do sistema (9.4) para $\lambda=0,1$. A região de estabilidade do ponto de equilíbrio assintoticamente estável $(-1,04 ; 0)$ é representada pela área em cinza claro. A componente conexa $D(0)$ do conjunto de nível $\left\{(x, y) \in \mathbb{R}^{2}: V_{\lambda_{0}}(x ; y)<0\right\}$ contendo o equilíbrio $(-1,04 ; 0)$ representada pela área em cinza escuro é uma estimativa uniforme da região de estabilidade perturbada $A_{\lambda}(-1,04 ; 0)$. 


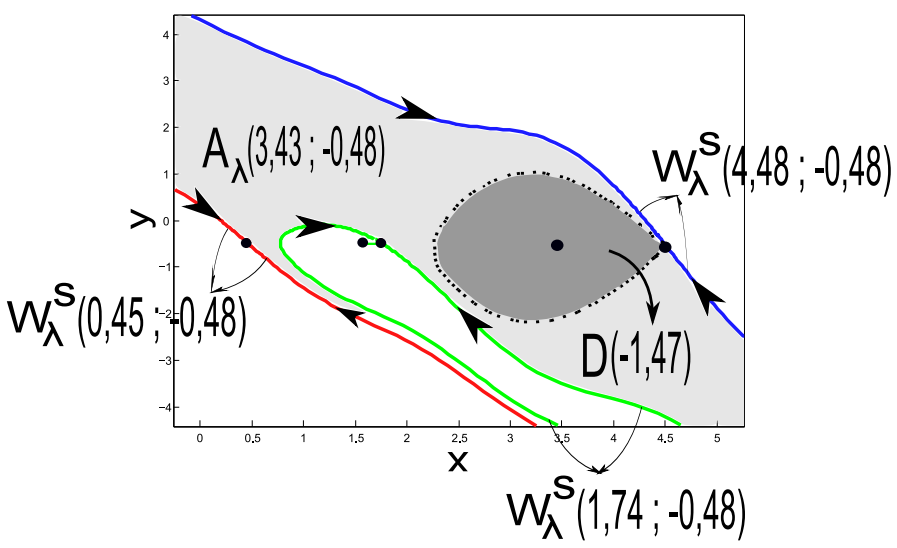

Figura 9.10: $O$ retrato de fase do (9.5) para $\lambda=0,48$. A região de estabilidade do ponto de equilíbrio assintoticamente estável $(3,43 ;-0,48)$ é representada pela área em cinza claro. A componente conexa $D(-1,47)$ do conjunto de nível $\left\{(x, y) \in \mathbb{R}^{2}: V_{\lambda}(x ; y)<\right.$ $-1,47\}$ contendo o equilíbrio $(3,43 ;-0,48)$ representada pela área em cinza escuro é uma estimativa da região de estabilidade $A_{\lambda}(3,43 ;-0,48)$.

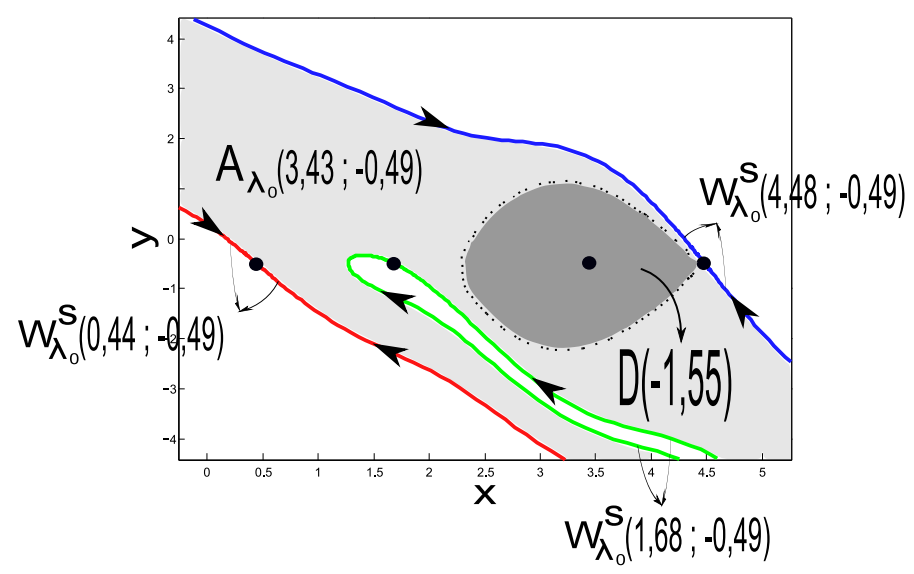

Figura 9.11: O retrato de fase do (9.5) para $\lambda_{0}=0,49$. A região de estabilidade do ponto de equilíbrio assintoticamente estável $(3,43 ;-0,49)$ é representada pela área em cinza claro. A componente conexa $D(-1,55)$ do conjunto de nível $\left\{(x, y) \in \mathbb{R}^{2}: V_{\lambda_{0}}(x ; y)<\right.$ $-1,55\}$ contendo o equilíbrio $(3,43 ;-0,49)$ representada pela área em cinza escuro é uma estimativa da região de estabilidade $A_{\lambda_{0}}(3,43 ;-0,49)$. 


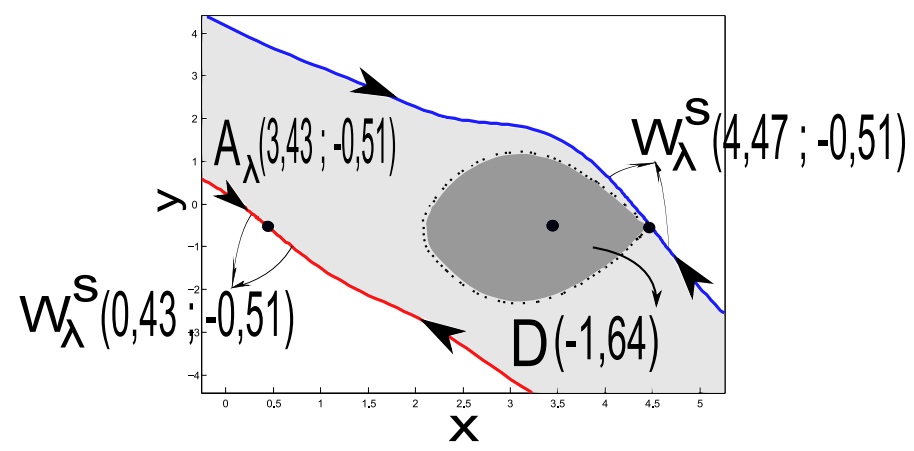

Figura 9.12: O retrato de fase do (9.5) para $\lambda=0,51$. A região de estabilidade do ponto de equilíbrio assintoticamente estável $(3,43 ;-0,51)$ é representada pela área em cinza claro. A componente conexa $D(-1,64)$ do conjunto de nível $\left\{(x, y) \in \mathbb{R}^{2}: V_{\lambda}(x ; y)<\right.$ $-1,64\}$ contendo o equilíbrio $(3,43 ;-0,51)$ representada pela área em cinza escuro é uma estimativa da região de estabilidade $A_{\lambda}(3,43 ;-0,51)$.

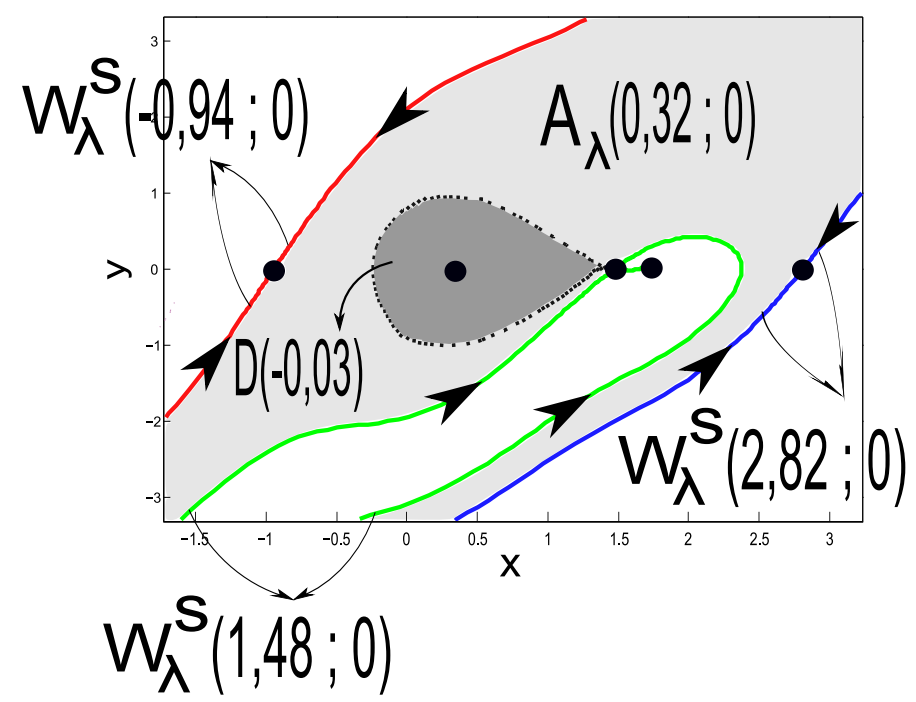

Figura 9.13: O retrato de fase do sistema (9.6) para $\lambda=-0,02$. A região de estabilidade do ponto de equilíbrio assintoticamente estável $(0,32 ; 0)$ é representada pela área em cinza claro. A componente conexa $D(-0,03)$ do conjunto de nível $\left\{(x, y) \in \mathbb{R}^{2}: V_{\lambda}(x ; y)<\right.$ $-0,03\}$ contendo o equilíbrio $(0,31 ; 0)$ representada pela área em cinza escuro é uma estimativa da região de estabilidade $A_{\lambda}(0,32 ; 0)$. 


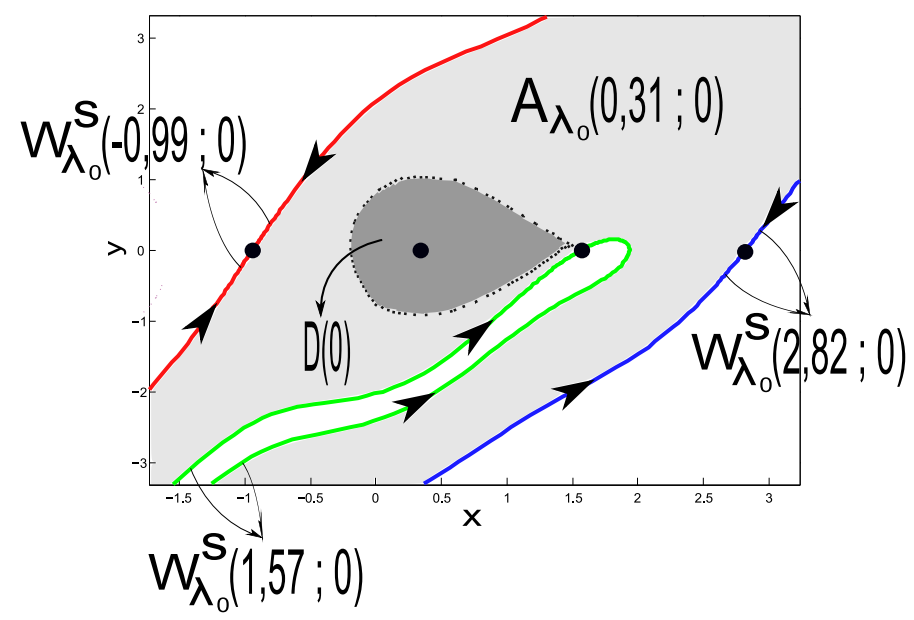

Figura 9.14: $O$ retrato de fase do sistema (9.6) para $\lambda_{0}=0$. A região de estabilidade do ponto de equilíbrio assintoticamente estável $(0,31 ; 0)$ é representada pela área em cinza claro. A componente conexa $D(0)$ do conjunto de nível $\left\{(x, y) \in \mathbb{R}^{2}: V_{\lambda_{0}}(x ; y)<0\right\}$ contendo o equilíbrio $(0,31 ; 0)$ representada pela área em cinza escuro é uma estimativa da região de estabilidade $A_{\lambda_{0}}(0,31 ; 0)$.

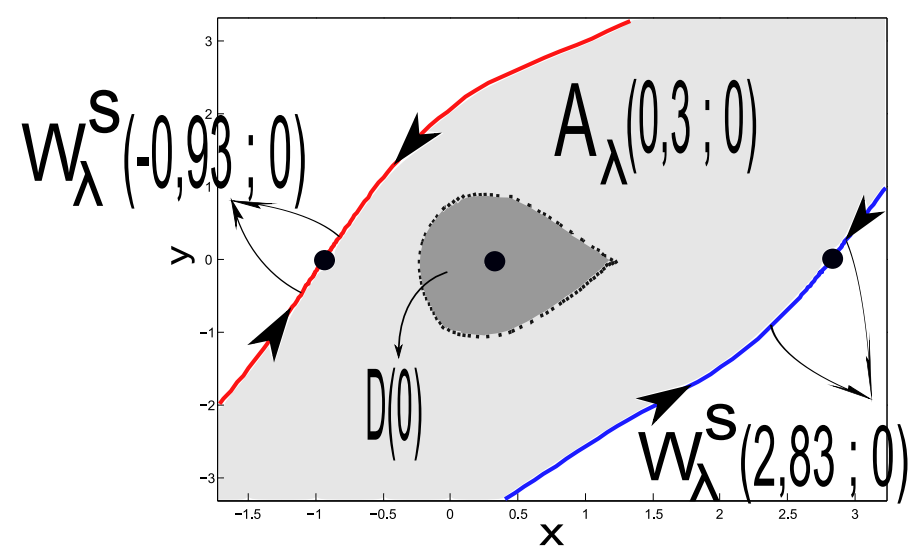

Figura 9.15: O retrato de fase do sistema (9.6) para $\lambda=0,02$. A região de estabi-lidade do ponto de equilíbrio assintoticamente estável $(0,3 ; 0)$ é representada pela área em cinza claro. A componente conexa $D(0)$ do conjunto de nível $\left\{(x, y) \in \mathbb{R}^{2}: V_{\lambda_{0}}(x ; y)<0\right\}$ contendo o equilíbrio $(0,3 ; 0)$ representada pela área em cinza escuro é uma estimativa uniforme da região de estabilidade perturbada $A_{\lambda}(0,3 ; 0)$. 


\section{CONCLUSÕES}

Neste trabalho, estudamos o comportamento da região de estabilidade de sistemas dinâmicos autônomos não lineares sujeitos a variações de parâmetros. Em particular, estudamos o comportamento da fronteira da região de estabilidade quando uma bifurcação sela-nó do tipo zero ocorre na fronteira da região de estabilidade.

Segundo nosso conhecimento, os resultados apresentados nesta tese são inéditos e estudam pela primeira vez o difícil problema de bifurcações da região de estabilidade de sistemas dinâmicos autônomos não lineares.

Caracterizamos a fronteira da região de estabilidade na presença de um ponto de equilíbrio sela-nó do tipo zero. Mostramos que a região de estabilidade e sua fronteira sofrem mudanças drásticas quando ocorre uma bifurcação sela-nó do tipo zero na fronteira. Em um certo sentido, mostramos que a região de estabilidade de um ponto de equilíbrio assintoticamente estável que se transforma em um ponto de equilíbrio sela-nó do tipo zero é "herdada" por outro ponto de equilíbrio assintoticamente estável. Em outras palavras, a região de estabilidade do ponto de equilíbrio assintoticamente estável que persiste é aumentada quando o parâmetro passa pelo parâmetro de bifurcação sela-nó do tipo zero. A região de estabilidade persistente "herda" a região de estabilidade do ponto de equilíbrio assintoticamente estável que desaparece na bifurcação sela-nó do tipo zero. Explorando a caracterização da fronteira da região de estabilidade propusemos estimativas ótimas da região de estabilidade via conjunto de nível de uma dada função energia na presença de um ponto de equilíbrio sela-nó do tipo zero na fronteira da região de estabilidade. Exibimos resultados que permitem entender o comportamento destas estimativas sob a influência das variações do parâmetro, incluindo variações do parâmetro próximo a um parâmetro de bifurcação sela-nó do tipo zero. Verificamos que quando o ponto de mínimo da função energia na fronteira da região de estabilidade é hiperbólico, o nível ótimo de energia para estimar a região de estabilidade perturbada é o nível de energia calculado no ponto de equilíbrio hiperbólico perturbado. Por outro lado, quando o ponto de mínimo da função energia na fronteira da região de estabilidade é um ponto de equilíbrio sela-nó do tipo zero, o nível ótimo de energia para estimar a região de estabilidade perturbada é calculado no ponto de equilíbrio instável, originado da bifurcação sela-nó do tipo zero, para 
$\lambda<\lambda_{0}$. Por outro lado, o nível de energia calculado no ponto de equilíbrio sela-nó do tipo zero fornece uma estimativa uniforme da região de estabilidade perturbada, $\lambda>\lambda_{0}$. Apresentamos um algoritmo conceitual para obter estimativas ótimas da região de estabilidade perturbada via conjunto de nível de uma dada função energia na vizinhança de um parâmetro de bifurcação sela-nó do tipo zero.

\subsection{Trabalhos Futuros}

Como sugestões para o desenvolvimento de trabalhos futuros nesta área, são propostas as seguintes questões:

- Caracterizar a fronteira da região de estabilidade na presença de um ponto de equilíbrio sela-nó do tipo $k \geqslant 1$ na fronteira da região de estabilidade;

- Estudar o comportamento da região de estabilidade e de sua fronteira sob a influência das variações do parâmetro nas vizinhanças de um parâmetro de bifurcação sela-nó do tipo $k \geqslant 1$;

- Propor um algoritmo conceitual para obter estimativas ótimas da região de estabilidade perturbada via conjunto de nível de uma dada função energia na vizinhança de um parâmetro de bifurcação sela-nó do tipo $k \geqslant 1$;

- Estudar outros tipos de bifurcações genéricas na fronteira da região de estabilidade; 


\section{REFERÊNCIAS}

ALBERTO, L. F. C. Caracterização e Estimativas da Área de Atração de Sistemas Dinâmicos Não-Lineares. São Carlos: Tese de Livre Docência, EESC-USP, 2006.

AMARAL, F. M.; ALBERTO, L. F. C. Stability boundary characterization of nonlinear autonomous dynamical systems in the presence of a type-zero saddle-node equilibrium point. Tendências em Matemática Aplicada, [S.1.], p.no prelo, 2010.

AMARAL, F. M.; ALBERTO, L. F. C. Stability Region Bifurcations of Nonlinear Autonomous Dynamical Systems: type-zero saddle-node bifurcations. International Journal of Robust and Nonlinear Control, [S.1.], p.no prelo, 2010.

ARROW, K. L.; HAHN, F. H. General Competitive Analysis. San Francisco: CA: Holden Day, 1971.

CHIANG, H.-D.; CHU, C.-C. Theorical foundation of the BCU method for direct stability analysis of network-Reduction power system models with small transfer conductances. IEEE Transactions on Circuits and Systems-I: Fundamental Theory and Applications, [S.1.], v.42, n.5, p.252-265, May 1995.

CHIANG, H. D.; HIRSCH, M. W.; WU, F. F. Stability regions of nonlinear autonomous dynamical systems. IEEE Trans. Automat. Control, [S.1.], v.33, n.1, p.16-27, Jan. 1988.

CHIANG, H.-D.; THORP, J. S. Stability Regions of Nonlinear Dynamical Systems: a constructive methodology. IEEE Transactions on Circuits and Systems-I: Fundamental Theory and Applications, [S.1.], v.34, n.12, p.1229-1241, December 1989.

CHIANG, H.-D.; WU, F. F.; VARAIYA, P. P. Foundations of direct methods for power system transient stability analysis. IEEE Transactions on Circuits and Systems-I, [S.1.], v.34, n.2, p.160-173, February 1987.

CHIANG, H. D.; WU, F. F.; VARAIYA, P. P. A BCU method for direct analysis of power system transient stability. IEEE Transactions on Power Systems, [S.1.], v.9, n.3, p.11941208, Ago 1992. 
CHIANG, H.-D.; WU, F.; VARAIYA, P. Foundations of the potential energy boundary surface method for power system transient stability analysis. , [S.1.], v.35, n.6, p.712-728, June 1988.

COUTINHO, D. F.; BAZANELlA, A. S.; TROFINO, A.; SILVA, A. S. Stability Analysis and Control of a Class of Differential-Algebraic Nonlinear Systems. International Journal of Robust and Nonlinear Control, [S.1.], v.14, n.16, p.1301-1326, 2004.

COUTINHO, D. F.; PAGANO, D. J.; TROFINO, A. On the Estimation of Robust Stability Regions for Nonlinear Systems with Saturation. SBA. Sociedade Brasileira de Automática, [S.1.], v.14, n.3, p.269-278, 2004.

COUTINHO, D. F.; SOUZA, C. E. de; TROFINO, A. Stability Analysis of Implicit Polynomial Systems. IEEE Trans. Automat. Control, [S.1.], v.54, n.5, p.10121018, 2009.

DEMARCO, C. L.; CANIZARES, C. A. A vector energy function approach for security analysis of AC/DC sysems. IEEE Transactions on Power Systems, [S.1.], v.7, n.3, p.1001-1011, 1992.

E. NOLDUS J. SPRIET, E. V.; CAUWENBERGHE, A. V. A New Lyapunov technique for stability analysis of chemical reactors. Automatica, [S.1.], v.10, p.675-680, 1974.

EL-ABIAD, A. H.; NAGAPPAN, K. Transient stability regions of multimachine power systems. IEEE Trans. Power Apparatus and Systems, [S.1.], v.85, p.169-179, February 1966.

GATTO, M.; RINALDI, S. Stability analysis of predator-prey models via the Lyapunov method. In: ANALYSIS AND COMPUTUTION OF EQUILIBRIA AND REGIONS OJ STABILITY, 1975. Anais... [S.1.: s.n.], 1975. p.139-153.

GENESIO, R.; TARTAGLIA, M.; VICINO, A. On the estimation of asymptotic stability regions: state of the art and new proposals. , [S.1.], v.30, n.8, p.747-755, Aug 1985.

GENESIO, R.; VICINO, A. New techniquess for constructing asymptotic stability regions for nonlinear systems. IEEE Transactions on Circuits and Systems, [S.1.], v.31, p.574581, June 1984.

GLESS, G. E. Direct Method of Lyapunov Applied to Transient Power System Stability. IEEE Trans. PAS, [S.1.], v.85, p.159-168, Feb. 1966.

GUCKENHEIMER, J.; HOLMES, P. Nonlinear Oscilations, Dynamical Systems and Bifurcations of Vector Fields. New York: Springer -Verlag, 1983. 
GUEDES, R. B. d. L.; ALBERTO, L. F. C.; BRETAS, N. G. Power System Low-Voltage Solutions Using an Auxiliary Gradient System for Voltage Collapse Purposes. IEEE Transactions on Power Systems, [S.1.], v.20, p.1528-1537, 2005.

GUILlEMIN, V.; POLLACK, A. Differential Topology. Englewood Cliffs: NJ: Prentice-Hall, 1974.

HALE, J. K. Ordinary Differential Equations. [S.1.]: J. Wiley, 1969.

HIRSCH, M. W.; PUGH, C. C.; SHUB, M. Invariant manifolds. Bulletin of the American Mathematical Society, [S.1.], v.76, n.5, p.1015-1019, 1970.

HUREWICZ., W.; WALLMAN, H. Dimension Theory. Princeton: NJ: Princeton University Press, 1948.

INFANTE, E. F.; CLARK, L. G. A method for the determination of domain of stability of second-order nonlinear autonomous systems. J. Appl. Mech., [S.1.], v.86, June 1964.

JOCIC, L. B. Planar regions of attraction. IEEE Trans. Automat. Contr., [S.1.], v.27, p.708-710, 1982.

KATO, T. Pertubation Theory for Linear Operators. Berlin Heidelberg: Springer Verlag, 1995.

LASALLE, J. P. The extent of asymptotic stability. In: NATIONAL ACADEMY OF SCIENCES, 1960. Proceedings... [S.1.: s.n.], 1960. p.363-365.

LASALLE, J. P. Some extensions of liapunov's second method. IRE Trans. on Circuit Theory, [S.1.], v.7, p.520-527, 1960.

LASALLE, J. P. Th Stability of Dynamical Systems. Philadelphia, Pennsylvania: Society for Industrial and Apllied Mathematics, 1976.

LASALLE, J. P.; LEFSCHETZ, S. Stability by Lyapunovs Direct Method with apllications. New York: Academic, 1961.

LEE, J.; CHIANG, H. D. Stability regions of non-hyperbolic dynamical systems: theory and optimal estimation. IEEE International Symposium on Circuits and Systems, [S.1.], p.28-31, May 2000.

LIMA, E. L. Curso de Análise. Rio de Janeiro: Projeto Euclides, 2006.

LOCCUFIER, M.; NOLDUS, E. A new trajectory reversing method for estimating stability regions of autonomous dynamic systems. Nonlinear Dyn., [S.1.], v.21, p.265-288, 2000. 
MAY, R. M. Stability and Complexity in Model Ecosystems. Princeton: NJ: Princeton University Press, 1973.

MUNKRES, J. R. Topology: a first course. London: Prentice-Hall, 2000.

N. KAKIMOTO, Y. O.; HAYASHI, M. Transient stability analysis of eletric power system via Lure-type Lyapunov function, Part I and Part II. Transactions IEE of Japan, [S.1.], v.98, p.516, 1978.

PAI, M. A. Power System Stability. Amsterdan: The Netherlands: North-Holland, 1981. PALIS, J. On Morse-Smale dynamical systems. Topology, [S.1.], v.8, p.385-405, 1969.

PALIS, J.; MELO, J. W. de. Introdução ao sistemas dinâmicos. IMPA-Rio de Janeiro: Projeto Euclides, 1977.

PEIXOTO, M. On an approximation theorem of Kupka and Smale. Differential Equations, [S.1.], v.3, p.214-227, 1967.

PERKO, L. Differential Equations and Dynamical Systems. New York: SpringerVerlag, 1991.

SARKAR, A. K. D.; RAO, N. D. Zubov's method and transient stability problems of power systems. Proc. Inst. Elec. Eng., [S.1.], v.118, p.1035-1040, August 1971.

SHUB, M. Global Stability of Dynamical Systems. New York: Springer-Verlag, 1987.

SILVA, F. H. J. R.; ALBERTO, L. F. C.; LONDON J. B. A., J.; BRETAS, N. G. Smooth perturbation on a classical energy function for lossy power system stability analysis. , [S.1.], v.52, n.1, p.222-229, Jan. 2005.

SILVA, F. H. J. R. d.; GUEDES, R. B. d. L.; ALBERTO, L. F. C.; BRETAS, N. G. Função Energia Generalizada de Controle para Estabilização de Sistemas Não Lineares. SBA. Sociedade Brasileira de Automática, [S.1.], v.20, p.133-145, 2009.

SMALE, S. Differentiable dynamical systems. Bulletin of the American Mathematical Society, [S.1.], v.73, p.747-817, 1967.

SMALE, S.; HIRSCH, M. Differential Equations, Dynamical Systems, and Linear Algebra. [S.1.]: Academic Press, 1974.

SMITH, K. T. Dynamics and Bifurcations. New York: Springer -Verlag, 1991.

SOTOMAYOR, J. Generic bifurcations of dynamical systems. In: PEIXOTO, M. M. (Ed.). Dinamical Systems. New York: Academic Press, 1973. p.549-560. 
SOTOMAYOR, J. Lições de equações diferenciais ordinárias. Rio de Janeiro: Projeto Euclides, 1979.

TSOLAS, N. A.; ARAPOSTATHIS, A.; VARAIYA, P. P. A Structure presereving energy function for power system transient stability analysis. IEEE Transactions on Circuits and Systems, [S.1.], v.32, n.10, p.1041-1049, October 1985.

VARAIYA, P.; WU, F. F.; CHEN, R.-L. Direct methods for transient stability analysis of power systems: recent results. , [S.1.], v.73, n.12, p.1703-1715, Dec. 1985.

VINOGRAD, V. E. The inadequacy of the method of characteristic exponents for the study of nonlinear differential equations. Math. Sbornik, [S.1.], v.41, p.431-438, 1957.

WIGGINS, S. Introduction to Applied Nonlinear Dynamical Systems and Chaos. New York: Springer- Verlag, 2003.

WILLEMS, J. L. Improved Lyapunov Function for Transient Power System Stability. In: IEE, 1968. Proceedings... [S.1.: s.n.], 1968. v.115, p.1315-1317.

YU, Y.; VONGSURIYA, K. Nonlinear power system stability study by Lyapunov function and Zubovs method. IEEE Trans. Power App. Syst., [S.1.], v.86, p.1480-1485, December 1967.

ZABORSZKY, J.; HUANG, G.; ZHENG, B.; LEUNG, T. C. On the phase portrait of a class of large nonlinear dynamic systems such as the power system. , [S.1.], v.33, n.1, p.4-15, Jan. 1988.

ZUBOV, V. I. Mathematical Methods for the Study of Automatic Control Sysfems. Israel: Jerusalem Academic Press, 1962.

ZUBOV, V. I. Methods of A.M. Lyapunov and Their Applicution. The Netherlands: Noordhoff, 1964.

ZUBOV, V. I. Stability of Motion. Berlin: Springer-Verlag, 1967. 